\title{
The emotional rollercoaster called Borderline Personality Disorder
}

Citation for published version (APA):

van Zutphen, C. P. (2017). The emotional rollercoaster called Borderline Personality Disorder: neural correlates of emotion regulation and impulsivity. [Doctoral Thesis, Maastricht University]. Maastricht University. https://doi.org/10.26481/dis.20170323lvz

Document status and date:

Published: 01/01/2017

DOI:

10.26481/dis.20170323lvz

Document Version:

Publisher's PDF, also known as Version of record

Document license:

Unspecified

\section{Please check the document version of this publication:}

- A submitted manuscript is the version of the article upon submission and before peer-review. There can be important differences between the submitted version and the official published version of record.

People interested in the research are advised to contact the author for the final version of the publication, or visit the DOI to the publisher's website.

- The final author version and the galley proof are versions of the publication after peer review.

- The final published version features the final layout of the paper including the volume, issue and page numbers.

Link to publication

\footnotetext{
General rights rights.

- You may freely distribute the URL identifying the publication in the public portal. please follow below link for the End User Agreement:

www.umlib.nl/taverne-license

Take down policy

If you believe that this document breaches copyright please contact us at:

repository@maastrichtuniversity.nl

providing details and we will investigate your claim.
}

Copyright and moral rights for the publications made accessible in the public portal are retained by the authors and/or other copyright owners and it is a condition of accessing publications that users recognise and abide by the legal requirements associated with these

- Users may download and print one copy of any publication from the public portal for the purpose of private study or research.

- You may not further distribute the material or use it for any profit-making activity or commercial gain

If the publication is distributed under the terms of Article $25 \mathrm{fa}$ of the Dutch Copyright Act, indicated by the "Taverne" license above, 


\section{The Emotional Rollercoaster called Borderline Personality Disorder}

Neural Correlates of Emotion Regulation and Impulsivity

Linda van Zutphen

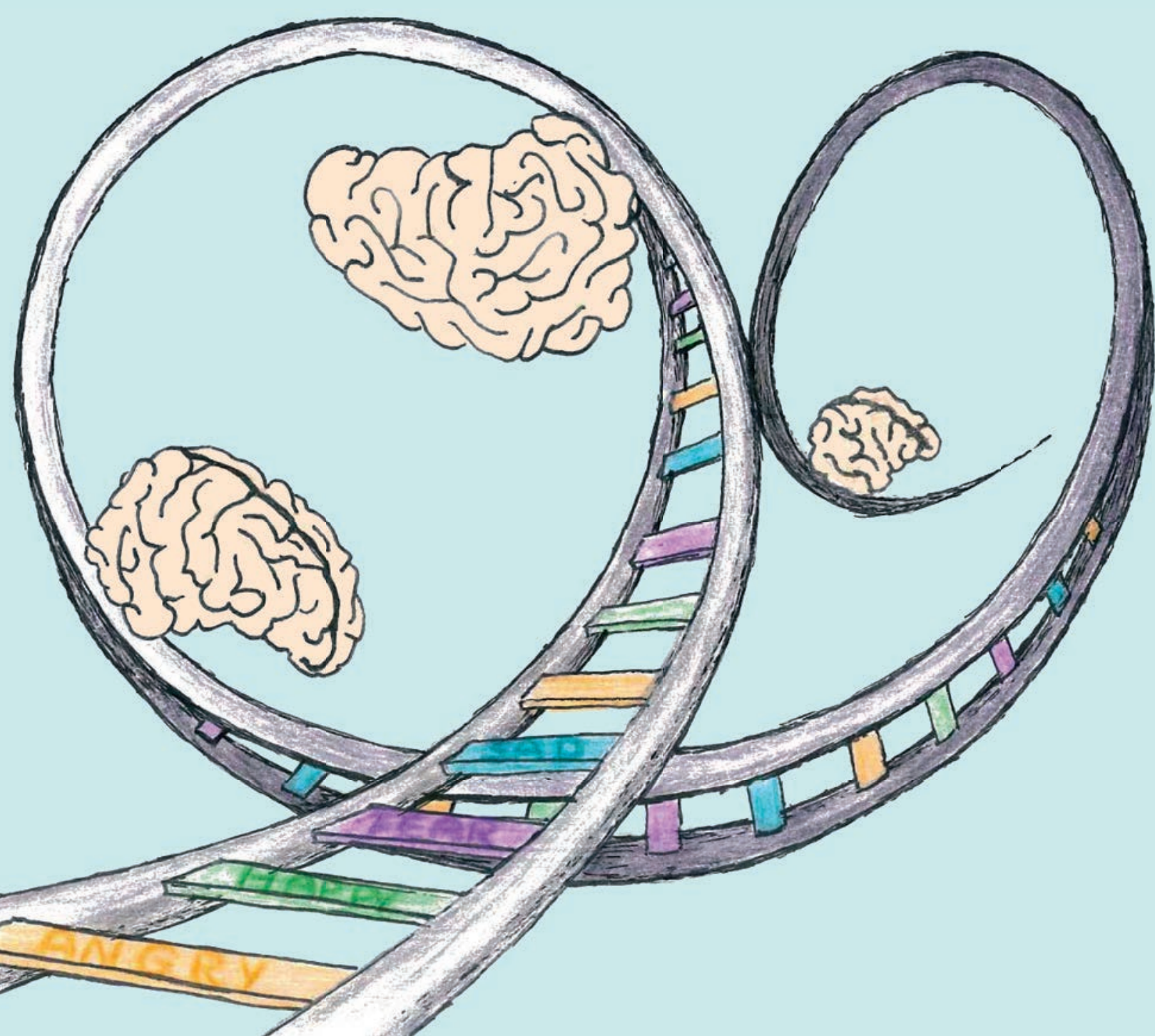





\section{The Emotional Rollercoaster called Borderline Personality Disorder}

Neural Correlates of Emotion Regulation and Impulsivity

Linda van Zutphen 


\section{Colophon}

Design cover picture: Marga, Francien \& Vincent van Zutphen

Lay-out:

Nikki Vermeulen | Ridderprint BV

Printing:

Ridderprint BV | www.ridderprint.nl

ISBN:

978-94-6299-515-4

๑) Copyright Linda van Zutphen, Maastricht 2017

Al rights are reserved. No part of this book may be reproduced or transmitted in any form or any means, without permission from the author, or, when appropriate, the publisher of the article. 


\title{
The Emotional Rollercoaster called Borderline Personality Disorder
}

Neural Correlates of Emotion Regulation and Impulsivity

\author{
Proefschrift
}

Ter verkrijging van de graad van doctor aan de Universiteit Maastricht, op gezag van de Rector Magnificus, Prof. Dr. Rianne M. Letschert, volgens het besluit van het College van Decanen,

in het openbaar te verdedigen op

Donderdag 23 maart 2017 om 16:00 uur

door

Carolina Petronella van Zutphen 


\section{Promotores}

Prof. dr. A.R. Arntz

Prof. dr. R. Goebel

\section{Copromotor}

Dr. N. Siep

\section{Beoordelingscommissie}

Prof. dr. Beatrice de Gelder (voorzitter)

Prof. dr. Bernet Elzinga (Leiden University)

Dr. Jill Lobbestael

Dr. Anne Roefs

Prof. dr. Christian Schmahl (Medical Faculty Mannheim/Heidelberg University, Germany)

The research presented in this dissertation was conducted in the program "Open Research Area in Social Sciences", supported by the Netherlands Organization for scientific Research (NWO), grant number 464-10-080 awarded to Prof. dr. R. Goebel, by the German Research Foundation, grant number JA1785/3-1 awarded to Dr. G.A. Jacob 


\section{Table of contents}

Chapter $1 \quad$ General Introduction 7

Chapter $2 \quad$ Emotional sensitivity, emotion regulation and 21 impulsivity in borderline personality disorder:

A critical review of $\mathrm{fMRI}$ studies

Chapter 3 Always on guard:

Emotion regulation in borderline personality disorder compared to non-patients and cluster-c personality disorder patients

Chapter 4 Impulse control under emotion processing:

an $\mathrm{fMRI}$ investigation in borderline personality disorder

compared to non-patients and cluster-c

personality disorder patients

Chapter 5 Deficient amygdala-prefrontal intrinsic connectivity after

effortful emotion regulation in borderline personality disorder

Chapter $6 \quad$ Summary and General discussion

Valorization Addendum

Samenvatting (Dutch Summary)

Dankwoord (Acknowledgements)

List of Publications 


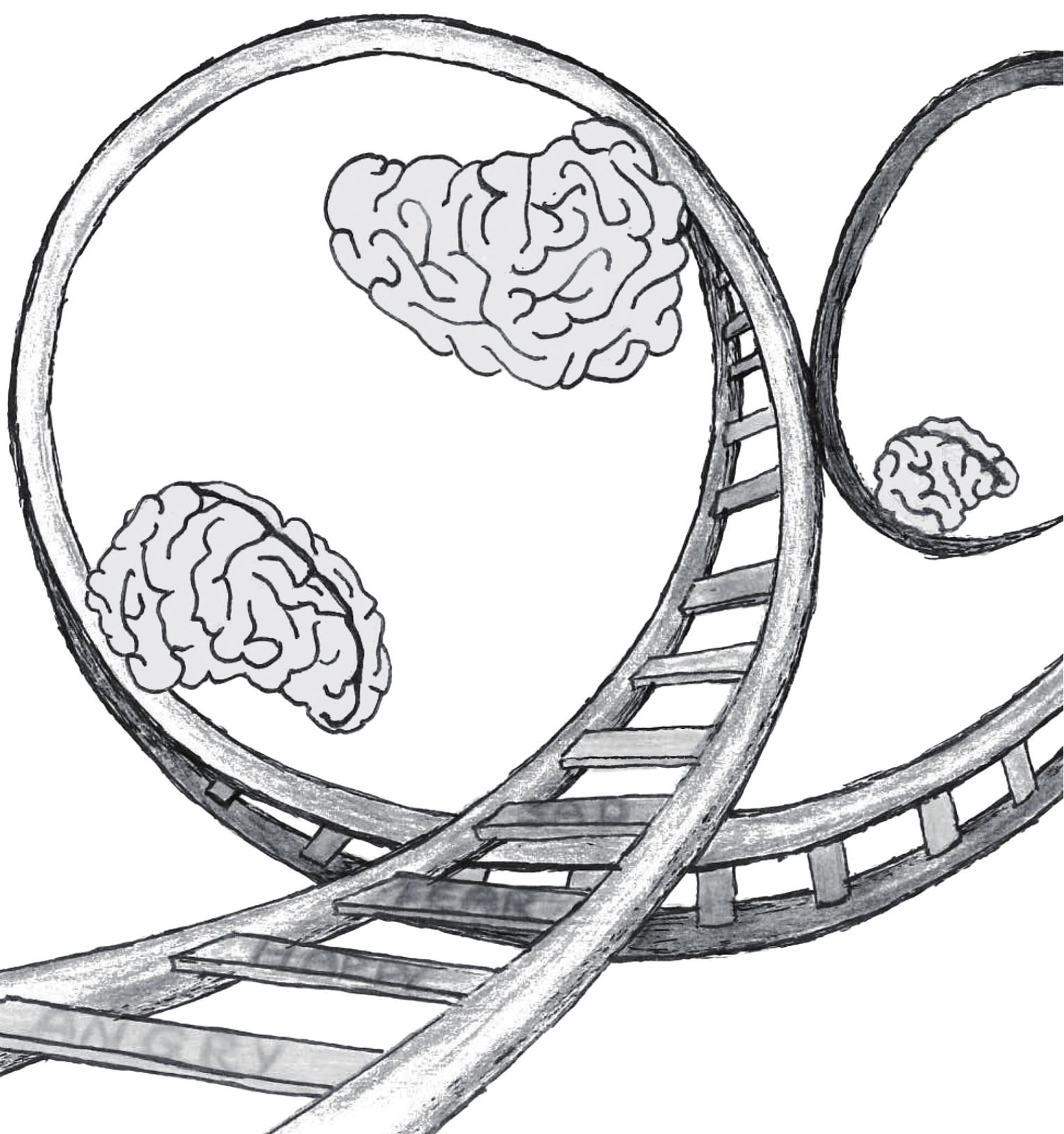


General Introduction 

Borderline personality disorder (BPD) is a severe mental disorder associated with high morbidity rates characterized by severe functional impairment and substantial treatment utilization, and high mortality rates including suicidal tendencies of which $10 \%$ succeeds (American Psychiatric Association, 2001). Epidemiological data show prevalence rates of 1-3\% of the general population (Lenzenweger, Lane, Loranger \& Kessler, 2007; Trull, Jahng, Tomko, Wood \& Sher, 2010), with up to 10\% of psychiatric outpatients and $20 \%$ in inpatients settings. In the mental health care settings BPD is three times more often diagnosed in females than in males (American Psychiatric Association, 2013; Lieb, Zanarini, Schmahl, Linehan \& Bohus, 2004). However, BPD might be missed in men, who may instead receive diagnoses of antisocial or narcissistic personality disorder, this difference in prevalence might reflect a gender bias. Yet, a recent study gives the impression that BPD is equally prevalent among females and males (Grant et al., 2008).

BPD severely affects functioning in the social and work domain and therefore poses challenges for those suffering from BPD, their families and mental health professionals. Beside unemployment and work-disability, BPD is considered to be difficult to treat; comprising intensive use of treatment and mental health care facilities, which result in a burden to public health systems and high costs to society (van Asselt, Dirksen, Arntz \& Severens, 2007).

\section{Evolution and Origin of BPD diagnosis}

The diagnosis of BPD has changed over the last century. Stern (1938) and Knight (1953) were the first who introduced the term borderline. BPD patients were described as suffering from a mild form of schizophrenia. This changed between the 1960 and 1970s, when BPD patients were viewed more as suffering from an affective disorder (e.g. depression or bipolar disorder). Kernberg (1967) defined borderline on a spectrum as the intermediate level of personality organization between psychotic personality organization and neurotic personality organization. As research showed that BPD was not a variation of schizophrenia and/or mood disorders, but differed in the sense of the course of the disorder (Gunderson, 2009), in the following decade BPD officially entered the psychiatric classification system as described in the Diagnostic and Statistical Manual of Mental Disorders (DSM-II) as Borderline Personality Disorder.

According to the current DSM-V, BPD is defined by a pervasive pattern of instability of emotions, interpersonal relationships, self-image and impulse control (American Psychiatric Association, 2013). The BPD specific symptomatology shows areas of deficits across four domains: A) disturbed emotion processing and emotion dysregulation including emotional instability, inappropriate anger and chronic feelings of emptiness (DSM-V criteria 6-8), B) behavioral dyscontrol, including impulse control and self-injury (DSM-V criteria 4 and 5), C) interpersonal deficits, including fear of abandonment and unstable relationships (DSM-V 
criteria 1 and 2), and D) maladaptive cognitive processes including identity disturbance and stress-related paranoid or dissociative symptoms (DSM-V criteria 3 and 9) (American Psychiatric Association, 2013; Lieb et al., 2004).

\section{Etiology of BPD}

Although BPD has attracted extensive clinical theorizing and research effort the etiology of BPD is still poorly understood. Here-to-date it is assumed that BPD evolves as an interaction between genetic factors as well as environmental factors. Empirical evidence for the role of genetic factors is sparse, but heritability is estimated at about 40\% (see for review Amad, Ramoz, Thomas, Jardri \& Gorwood, 2014). The most important environmental factors involved in the etiology of BPD are insecure attachment styles and childhood maltreatment. Various types of physical and emotional abuse or neglect, including traumatic experiences as abandonment and sexual abuse, during childhood are frequently reported (Lobbestael, Arntz \& Bernstein, 2010; Zanarini et al., 2002). No close association between either biological (e.g. specific genes) or psychosocial (e.g. aversive childhood events) and the development of BPD has been identified, therefore a biopsychosocial model will probably provide the best explanation for the etiology of BPD.

\section{Cognitive Perspective}

In the cognitive perspective on BPD, an essential role is given to the patient's maladaptive beliefs or schema's that are assumed to underlie the patient's dysfunctional behavior, thoughts, feelings and emotions (Beck \& Freeman, 1990). Maladaptive beliefs and schemas are overgeneralized, inflexible and considered hypervigilant when the threshold for its activation by a trivial trigger such as threat or danger is low. Three disturbed cognitive schemata are proposed to play a central role in BPD: "The world is (and others are) dangerous and malevolent", "I am powerless and vulnerable" and "I am inherently bad and unacceptable" (Arntz, Dreessen, Schouten \& Weertman, 2004; Pretzer, 1990). Additionally, dichotomous thinking (i.e. all-or-nothing or black-and-white thinking) is considered as a cognitive error for BPD.

Important factors in the development and maintenance of these maladaptive beliefs and schemas are biased cognitive information processing such as attention, interpretation and memory biases. A recent review on cognitive biases in BPD (Baer, Peters, Eisenlohr-Moul, Geiger \& Sauer, 2012) found that BPD patients show an attentional bias towards negative stimuli, which occurred in response to general negative stimuli, and responses were similar to various other clinical control groups, indicating that results were not specific for BPD related themes and BPD diagnosis. Furthermore, studies with regard to an interpretation bias in BPD show that BPD patients rate neutral or ambiguous interpersonal stimuli more 
negative (Baer et al., 2012). Finally, empirical literature shows a memory bias for BPD patients for either general negative as well as for BPD-related stimuli (Baer et al., 2012). The study of this maladaptive information processes might give insight in understanding BPD and be helpful to improve treatment.

\section{Neurobiological Perspective}

There is a widespread consensus that essential clinical features of BPD are emotional dysregulation and impulsivity, especially within an interpersonal context. Both features therefore have been the focus of empirical studies, and repeatedly identified as phenotypic traits of BPD (Siever \& Davis, 1991). One of the leading theories (Koenigsberg et al., 2009b; Linehan, 1993; Sebastian, Jacob, Lieb \& Tüscher, 2013a) proposes that BPD is best characterized as an emotional dysregulation disorder. The core of the emotion processing difficulties within BPD might be two-fold, with increased sensitivity on one hand and inability to regulate emotions on the other hand. Emotional sensitivity can be defined by a lower threshold to detect or respond to emotional stimuli. In other words, stimuli are more likely to be experienced as emotional. This might lead to hyperawareness to emotional stimuli and more frequent or intense emotional experiences. A common approach to examine emotional sensitivity is to expose participants to emotional challenges to provoke emotional responses. This can be done by means of the presentation of facial expressions, emotional scenes or (personalized) emotional stories, or a specific emotion-related task like an emotional discrimination task. Emotion regulation refers to the ability of individuals to influence the expression and experience of their emotions (Gross, 1998, 2007). This can be accomplished by means of cognitive control, such as reappraisal. Reappraisal refers to the ability to cognitively re-evaluate a stimulus or situation, and modulate a possible response while the emotion is experienced. Consequently, this allows us to decrease emotional experience. Therefore, the ability to regulate emotions has an impact on our mental and physical well-being (Gross, 1998, 2007). In other words, it makes ourselves feel better or worse. The first study that investigated the neural circuits of reappraisal of negative scenes in non-patients was performed by Ochsner et al. (2002). Two conditions were included in their regulation paradigm: 'attend' (experience naturally emotional state) and 'reappraise' (interpret pictures in a way that they no longer feel negative). Because both conditions involve attention to emotion, brain areas more active during reappraising reflect cognitive control of emotion, whereas brain areas more active during attending comprise emotional responding. According to the model of cognitive control of emotion (Ochsner, Silvers \& Buhle, 2012) brain areas involved in emotion generation are the amygdala, ventral striatum, anterior insula and orbitofrontal cortex. Brain areas most frequently associated with emotion regulation are the dorsolateral prefrontal cortex, ventrolateral prefrontal cortex, dorsomedial prefrontal cortex and dorsal anterior cingulate cortex (Ochsner et al., 2012; Phan et al., 2005). 
Emotion dysregulation might result in impulsive behavior, especially when under emotional conditions. Impulsivity is a broad and multidimensional concept and this leads to a diversity of definitions of impulsivity in the clinical domain (Evenden, 1999; Moeller, Barratt, Dougherty, Schmitz \& Swann, 2001). The pattern of impulsivity often diagnosed in BPD patients is associated within the behavioral domain, and defined as a predisposition of rapidly and unplanned responses without thinking and considering the consequences (Moeller et al., 2001). Therefore, impulsivity is often related to response inhibition. This pattern of disinhibition in BPD is characterized by physical self-destructive behavior such as self-mutilation and suicide attempts, and more general forms of impulsivity like excessive spending, unsafe sex, substance abuse, binge eating, reckless driving, substance abuse, verbal outburst and reactive aggression. To examine response inhibition the Stop signal and Go/no-go tasks are commonly applied to measure behavioral inhibition. Key brain areas associated with response inhibition are the inferior frontal gyrus/ventrolateral prefrontal cortex, dorsal anterior cingulate cortex, dorsolateral prefrontal cortex, ventromedial prefrontal cortex, inferior parietal lobe, pre-supplementary motor area, thalamus, dorsal striatum and nucleus subthalamicus (Aron, 2007; Garavan, Ross \& Stein, 1999; Horn, Dolan, Elliott, Deakin \&Woodruff, 2003; Sebastian et al., 2013b; Simmonds, Pekar \& Mostofsky, 2008; Swick, Ashley \& Turken, 2011).

Taken together, emotion dysregulation as well as impulsivity in BPD might both be considered as a dysfunction of the interaction between the underlying inhibitory mechanism and emotion, in which emotion dysregulation can be considered as cognitive inhibition referring to the ability to ignore irrelevant stimuli, and impulsivity is related to behavioral inhibition referring to the inhibition of motor responses.

\section{Investigating BPD: Functional Neuroimaging}

To test the differentiation between the inhibitory and emotional systems functional magnetic resonance imaging (fMRI) is a useful tool, because both processes involve brain areas that are spatially differently located in the brain. Particularly, the bottom-up limbic system (e.g. amygdala and anterior insula) is involved in emotion generation and the prefrontal areas are involved in top-down regulatory and inhibitory processes (dorsolateral, dorsomedial, ventrolateral prefrontal cortices and dorsal anterior cingulate cortex) (Phillips, Drevets, Rauch \& Lane, 2003).

FMRI gives useful insight in understanding the brain and is the primary research method of present dissertation. It is non-invasive and allows studying and localizing certain cognitive processes in the brain. Like muscles, neurons in the brain need oxygen when they are working. FMRI is based on the assumption that locally increased energy demands, caused by neurons firing, will result in more cerebral blood flow. Blood with a lot of oxygen in it has a different MRI signal than blood with less oxygen in it, and therefore blood-oxygen-level 
dependent (BOLD) signal changes represent a vascular coupling to neural activation.

A growing number of fMRI studies in BPD using emotion provocation paradigms highlight a dysfunctional fronto-limbic network comprised of increased limbic activity, involved in emotion generation, and a decreased activity in the prefrontal cortex, involved in regulatory and inhibitory processes (see reviews Krause-Utz, Winter, Niedtfeld \& Schmahl, 2014; Mauchnik \& Schmahl, 2010; Schulze, Schmahl \& Niedtfeld, 2015).

\section{Psychological Treatment}

Long-term psychotherapy is currently the primary treatment for BPD (Zanarini, 2009). The four most prominent psychological treatments for BPD are: Dialectical behavior therapy, Schema therapy, Mentalization-based therapy and Transference-focused psychotherapy. Dialectical behavior therapy and Schema therapy are based on cognitive-behavioral principles in which new learning experiences and self-management skills are acquired, whereas Mentalization-based therapy and Transference-focused psychotherapy are based on psychodynamic principles in which manifestations of the pathology in the therapeutic relationship (transference) are central. Dialectical behavior therapy is an adaptation of cognitive-behavioral therapy, which targets emotion dysregulation and was specifically designed to reduce self-injury and suicide attempts (Linehan, 1993). It combines principles of cognitive therapy and mindfulness, and in addition aims to change stress tolerance and interpersonal behavior. Schema therapy combines techniques from cognitive-behavioral, psychoanalytic and attachment theories, and helps to identify unmet basic emotional needs during childhood and replaces maladaptive coping styles (Young, Klosko \&Weishaar, 2003). Mentalization-based therapy is derived from the concept that BPD patients suffer from disorganized attachment and need to learn to 'mentalize' (Bateman \& Fonagy, 2004). In order to improve emotion regulation and interpersonal relationships it aims to increase the mentalizing capacity and recognize the feelings in others and those they experience themselves. Finally, transference-focused psychotherapy integrates the distorted perception of the self and significant others and undifferentiated affect (Kernberg, Yeomans, Clarkin \& Levy, 2008). The primary goal is to reduce self-destructive behavior (e.g. facilitate better impulse control) through clarification, confrontation and transference interpretation techniques. These four psychological treatments have been proven effective in BPD, nonetheless shorter less intensive and less costly versions of these treatments are needed to increase accessibility and to relieve the burden on patients and therapists (Zanarini, 2009).

\section{Aim of Present Dissertation}

Present neuroimaging findings concerning the neurobiological etiology of BPD are inconclusive and inconsistent (Chapter 2) and some important issues should be addressed 
to improve our understanding of BPD. First, studies in BPD on facial recognition report evidence of biased perception towards heightened sensitivity and difficulty recognizing for a range of negative facial expressions (social signals of threat and rejection), and a tendency to attribute ambiguous and neutral faces more negatively (see for reviews Daros, Zakzanis \& Ruocco, 2013; Mitchell, Dickens \& Picchioni, 2014). Based on these results it makes sense that most research focused on negative stimuli, however it remains unclear whether the increased emotional sensitivity is restricted to negative emotions or whether it also hold for other valences such as positive stimuli, pointing to a general emotional sensitivity. Nearly all previous studies assessed responses during the confrontation solely with negative stimuli (contrasted with neutral stimuli or fixation). Further research is needed to examine whether deficits are only to negative emotions, or whether they also involve other types of emotions. Second, BPD has high comorbidity with other Axis I and Axis II disorders, especially mood, anxiety (e.g. posttraumatic stress disorder), substance use, eating and Cluster-C personality disorders (i.e. avoidant, dependent and obsessive-compulsive personality disorder) (McGlashan et al., 2000; Zanarini et al., 1998a, 1998b; Zimmerman \& Mattia, 1999). Hence it remains uncertain whether findings are specific for BPD or characteristic of psychopathology in general, as previous studies lack a clinical control group. Third, most studies were statistically underpowered, which could be one of the main causes of the inconsistency in reported findings. For instance, it is interesting that the focus of many fMRI studies was on hyperactivity of the amygdala, an area important for emotion generation, however some studies did not find this increased response (Guitart-Masip et al., 2009; Koenigsberg et al., 2009b), while other only showed an amygdala response after a region-of-interest or small volume correction (Koenigsberg et al., 2009a; Schulze et al., 2011).

The present thesis covers the results of several studies examining the neural correlates of emotional sensitivity, emotion regulation (Chapter $3 \& 5$ ) and impulsivity (Chapter 4) in BPD. The aim is to investigate BPD uniqueness with regard to especially stimulus category specificity and diagnosis specificity.

\section{Outline of the Dissertation}

Chapter 2 of this dissertation starts with a detailed introduction of the neurobiological model of BPD, summarizing fMRI studies examining emotional sensitivity, emotion regulation and impulsivity in BPD. Following, shedding a critical light on important unanswered issues and topics for consideration to improve future research.

In order to improve our understanding of BPD, in Chapter $\mathbf{3}$ and $\mathbf{4}$ we aimed to address some of the issues hinted above and discussed in the critical review of Chapter 2. More specifically our objective is to study BPD uniqueness of neural correlates of emotional sensitivity, emotion regulation and impulsivity. We focus on the generalizability across stimulus categories, and diagnosis compared to other personality disorders. In addition, 
because of low statistical power in previous fMRI studies in BPD we increased the statistical power by increasing the sample size recruited from five different international institutes. It was hypothesized that BPD patients would show higher activity in emotion generation brain areas (e.g. amygdala and anterior insula) when passive viewing or inhibiting responses of emotional stimuli, compared to non-patients and Cluster-C personality disorder patients. Additionally, we expected that BPD patients would show dysregulated activity in regulatory or inhibitory brain areas (e.g. dorsolateral prefrontal cortex, ventrolateral prefrontal cortex and dorsal anterior cingulate cortex), whilst regulating or inhibiting responses of emotional stimuli, compared to non-patients and Cluster-C personality disorder patients. If brain responses in BPD would be specific towards negative stimuli and diagnosis specific for BPD, then a difference should be shown in the reported brain areas for positive and erotic stimuli, or compared to the Cluster-C personality disorder group.

FMRI is not only suitable to localize structures involved in mental operations, but also to study connectivity within and between brain networks. By means of functional connectivity one would be able to examine an interaction between brain regions rather than investigating brain areas in isolation. Large networks of brain structures preform a task together; one region receives input from other regions and sends output to yet other regions. In Chapter $\mathbf{5}$ we explored enduring effects of emotion regulation, by measuring whether the emotion regulation paradigm from Chapter 3 induces changes of the connectivity between the amygdala and prefrontal regions after the task during a task-free resting-state of the brain. It was expected that BPD patients would exhibit a weaker increase of resting-state functional connectivity between the amygdala and prefrontal regions after the task, compared to nonpatients, promoting difficulties with emotion regulation.

Chapter 6 summarizes and discusses the findings of this dissertation. Furthermore, methodological issues, theoretical and clinical implications, and recommendations for future research will be considered.

In the valorization addendum a broader societal and economic relevance will be described. 


\section{References}

Amad, A., Ramoz, N., Thomas, P., Jardri, R., \& Gorwood, P. (2014). Genetics of borderline personality disorder: systematic review and proposal of an integrative model. Neuroscience and Biobehavioral Reviews, 40, 6-19. doi: 10.1016/j.neubiorev.2014.01.003

American Psychiatric Association. (2001). Practice guideline for the treatment of patients with borderline personality disorder. American Psychiatric Association. The American Journal of Psychiatry, 158, 1-52.

American Psychiatric Association. (2013). Diagnostic and Statistical Manual of Mental Disorder (5th ed.). Arlington, VA: American Psychiatric Association.

Arntz, A., Dreessen, L., Schouten, E., \&Weertman, A. (2004). Beliefs in personality disorders: a test with the personality disorder belief questionnaire. Behaviour Research and Therapy, 42, 1215-1225. doi: 10.1016/j.brat.2003.08.004

Aron, A. R. (2007). The neural basis of inhibition in cognitive control. Neuroscientist, 13, 214-228. doi: $10.1177 / 1073858407299288$

Baer, R. A., Peters, J. R., Eisenlohr-Moul, T. A., Geiger, P. J., \& Sauer, S. E. (2012). Emotion-related cognitive processes in borderline personality disorder: a review of the empirical literature. Clinical Psychology Review, 32, 359-369. doi: 10.1016/j.cpr.2012.03.002

Bateman, A., \& Fonagy, P. (2004). Psychotherapy for Borderline Personality Disorder: Mentalization Based Treatment. Oxford: Oxford University Press.

Beck, A. T., \& Freeman, A. (1990). Cognitive therapy of personality disorders. New York: Guilford Press.

Daros, A. R., Zakzanis, K. K., \& Ruocco, A. C. (2013). Facial emotion recognition in borderline personality disorder. Psychological Medicine, 43, 1953-1963. doi: 10.1017/S0033291712002607

Evenden, J. L. (1999). Varieties of impulsivity. Psychopharmacology, 146, 348-361.

Garavan, H., Ross, T. J., \& Stein, E. A. (1999). Right hemispheric dominance of inhibitory control: an event-related functional MRI study. Proceedings of the National Academy of Sciences of the United States of America, 96, 83018306. doi: 10.1073/pnas.96.14.8301

Grant, B. F., Chou, S. P., Goldstein, R. B., Huang, B., Stinson, F. S., Saha, T. D., .. Ruan, W. J. (2008). Prevalence, correlates, disability, and comorbidity of DSM-IV borderline personality disorder: results from the Wave 2 National Epidemiologic Survey on Alcohol and Related Conditions. The Journal of Clinical Psychiatry, 69, 533-545.

Gross, J. J. (1998). The emerging field of emotion regulation: an integrative review. Review of General Psychology, 2, 271-299. doi: 10.1037//1089-2680.2.3.271

Gross, J. J. (2007). Handbook of Emotion Regulation. New York, NY: Guilford Publications

Guitart-Masip, M., Pascual, J. C., Carmona, S., Hoekzema, E., Berge, D., Perez, V., . . Vilarroya, O. (2009). Neural correlates of impaired emotional discrimination in borderline personality disorder: an fMRI study. Progress in Neuro-psychopharmacology \& Biological Psychiatry, 33, 1537-1545. doi: 10.1016/j.pnpbp.2009.08.022

Gunderson, J. G. (2009). Borderline personality disorder: ontogeny of a diagnosis. The American Journal of Psychiatry, 166, 530-539. doi: 10.1176/appi.ajp.2009.08121825

Horn, N. R., Dolan, M., Elliott, R., Deakin, J. F., \& Woodruff, P. W. (2003). Response inhibition and impulsivity: an fMRI study. Neuropsychologia, 41, 1959-1966. doi: 10.1016/S0028-3932(03)00077-0

Kernberg, O. (1967). Borderline personality organization. Journal of the American Psychoanalytic Association, 15, 641685.

Kernberg, O. F., Yeomans, F. E., Clarkin, J. F., \& Levy, K. N. (2008). Transference focused psychotherapy: overview and update. The International Journal of Psycho-analysis, 89, 601-620. doi: 10.1111/j.1745-8315.2008.00046.x

Knight, R. P. (1953). Borderline states. Bulletin of the Menninger Clinic, 17, 1-12.

Koenigsberg, H. W., Fan, J., Ochsner, K. N., Liu, X., Guise, K. G., Pizzarello, S., . . Siever, L. J. (2009a). Neural correlates of the use of psychological distancing to regulate responses to negative social cues: a study of patients with borderline personality disorder. Biological Psychiatry, 66, 854-863. doi: 10.1016/j.biopsych.2009.06.010

Koenigsberg, H. W., Siever, L. J., Lee, H., Pizzarello, S., New, A. S., Goodman, M., . . Prohovnik, I. (2009b). Neural correlates of emotion processing in borderline personality disorder. Psychiatry Research, 172, 192-199. doi: 10.1016/j.pscychresns.2008.07.010

Krause-Utz, A., Winter, D., Niedtfeld, I., \& Schmahl, C. (2014). The latest neuroimaging findings in borderline personality disorder. Current Psychiatry Reports, 16, 438. doi: 10.1007/s11920-014-0438-z 
Lenzenweger, M. F., Lane, M. C., Loranger, A. W., \& Kessler, R. C. (2007). DSM-IV personality disorders in the National Comorbidity Survey Replication. Biological Psychiatry, 62, 553-564. doi: 10.1016/j.biopsych.2006.09.019

Lieb, K., Zanarini, M. C., Schmahl, C., Linehan, M. M., \& Bohus, M. (2004). Borderline personality disorder. Lancet, 364, 453-461. doi: 10.1016/S0140-6736(04)16770-6

Linehan, M. M. (1993). Cognitive-Behavioral Treatment of Borderline Personality Disorder. New York: Guilford Press.

Lobbestael, J., Arntz, A., \& Bernstein, D. P. (2010). Disentangling the relationship between different types of childhood maltreatment and personality disorders. Journal of Personality Disorders, 24, 285-295. doi: 10.1521/ pedi.2010.24.3.285

Mauchnik, J., \& Schmahl, C. (2010). The latest neuroimaging findings in borderline personality disorder. Current Psychiatry Reports, 12, 46-55. doi: 10.1007/s11920-009-0089-7

McGlashan, T. H., Grilo, C. M., Skodol, A. E., Gunderson, J. G., Shea, M. T., Morey, L. C., .. Stout, R. L. (2000). The Collaborative Longitudinal Personality Disorders Study: baseline Axis I/II and II/II diagnostic co-occurrence. Acta Psychiatrica Scandinavica, 102, 256-264. doi: 10.1034/j.1600-0447.2000.102004256.x

Mitchell, A. E., Dickens, G. L., \& Picchioni, M. M. (2014). Facial emotion processing in borderline personality disorder: a systematic review and meta-analysis. Neuropsychological Review, 24, 166-184. doi: 10.1007/s11065-014-9254-9

Moeller, F. G., Barratt, E. S., Dougherty, D. M., Schmitz, J. M., \& Swann, A. C. (2001). Psychiatric aspects of impulsivity. The American Journal of Psychiatry, 158, 1783-1793. doi: 10.1176/appi.ajp.158.11.1783

Ochsner, K. N., Bunge, S. A., Gross, J. J., \& Gabrieli, J. D. (2002). Rethinking feelings: an fMRI study of the cognitive regulation of emotion. Journal of Cognitive Neuroscience, 14, 1215-1229. doi: 10.1162/089892902760807212

Ochsner, K. N., Silvers, J. A., \& Buhle, J.T. (2012). Functional imaging studies of emotion regulation: a synthetic review and evolving model of the cognitive control of emotion. Annals of the New York Academy of Sciences, 1251, E124. doi: 10.1111/j.1749-6632.2012.06751.x

Phan, K. L., Fitzgerald, D. A., Nathan, P. J., Moore, G. J., Uhde, T. W., \& Tancer, M. E. (2005). Neural substrates for voluntary suppression of negative affect: a functional magnetic resonance imaging study. Biological Psychiatry, 57, 210219. doi: 10.1016/j.biopsych.2004.10.030

Pretzer, J. (1990). Borderline personality disorder. In A. T. Beck \& A. Freeman (Eds.), Cognitive therapy of personality disorders (pp. 176-207). New York/London: Guilford Press.

Schulze, L., Domes, G., Kruger, A., Berger, C., Fleischer, M., Prehn, K., ... Herpertz, S. C. (2011). Neuronal correlates of cognitive reappraisal in borderline patients with affective instability. Biological Psychiatry, 69, 564-573. doi: 10.1016/j.biopsych.2010.10.025

Schulze, L., Schmahl, C., \& Niedtfeld, I. (2015). Neural correlates of disturbed emotion processing in borderline personality disorder: a multimodal meta-analysis. Biological Psychiatry. doi: 10.1016/j.biopsych.2015.03.027

Sebastian, A., Jacob, G., Lieb, K., \& Tüscher, O. (2013a). Impulsivity in borderline personality disorder: a matter of disturbed impulse control or a facet of emotional dysregulation? Current Psychiatry Reports, 15, 339. doi: 10.1007/s11920-012-0339-y

Sebastian, A., Pohl, M. F., Kloppel, S., Feige, B., Lange, T., Stahl, C., ... Tüscher, O. (2013b). Disentangling common and specific neural subprocesses of response inhibition. Neurolmage, 64, 601-615. doi: 10.1016/j. neuroimage.2012.09.020

Siever, L. J., \& Davis, K. L. (1991). A psychobiological perspective on the personality disorders. The American Journal of Psychiatry, 148, 1647-1658.

Simmonds, D. J., Pekar, J. J., \& Mostofsky, S. H. (2008). Meta-analysis of Go/No-go tasks demonstrating that fMRI activation associated with response inhibition is task-dependent. Neuropsychologia, 46, 224-232. doi: 10.1016/j. neuropsychologia.2007.07.015

Stern, A. (1938). Psychoanalytic investigation and therapy in the borderline group of neuroses. The Psychoanalytic Quarterly, 7, 467-489.

Swick, D., Ashley, V., \& Turken, U. (2011). Are the neural correlates of stopping and not going identical? Quantitative meta-analysis of two response inhibition tasks. Neurolmage, 56, 1655-1665. doi: 10.1016/j. neuroimage.2011.02.070

Trull, T. J., Jahng, S., Tomko, R. L., Wood, P. K., \& Sher, K. J. (2010). Revised NESARC personality disorder diagnoses: gender, prevalence, and comorbidity with substance dependence disorders. Journal of Personality Disorders, 24, 412-426. doi: 10.1521/pedi.2010.24.4.412 
van Asselt, A. D., Dirksen, C. D., Arntz, A., \& Severens, J. L. (2007). The cost of borderline personality disorder: societal cost of illness in BPD-patients. European Psychiatry, 22, 354-361. doi: 10.1016/j.eurpsy.2007.04.001

Young, J. E., Klosko, J. S., \& Weishaar, M. E. (2003). Schema Therapy: A Practitioner's Guide. New York: Guilford Press.

Zanarini, M. C. (2009). Psychotherapy of borderline personality disorder. Acta Psychiatrica Scandinavica, 120, 373377. doi: 10.1111/j.1600-0447.2009.01448.x

Zanarini, M. C., Frankenburg, F. R., Dubo, E. D., Sickel, A. E., Trikha, A., Levin, A., \& Reynolds, V. (1998a). Axis I comorbidity of borderline personality disorder. The American Journal of Psychiatry, 155, 1733-1739.

Zanarini, M. C., Frankenburg, F. R., Dubo, E. D., Sickel, A. E., Trikha, A., Levin, A., \& Reynolds, V. (1998b). Axis II comorbidity of borderline personality disorder. Comprehensive Psychiatry, 39, 296-302. doi: 10.1016/S0010-440X(98)90038-4

Zanarini, M. C., Yong, L., Frankenburg, F. R., Hennen, J., Reich, D. B., Marino, M. F., \& Vujanovic, A. A. (2002). Severity of reported childhood sexual abuse and its relationship to severity of borderline psychopathology and psychosocial impairment among borderline inpatients. The Journal of Nervous and Mental Disease, 190, 381 387. doi: 10.1097/00005053-200206000-00006

Zimmerman, M., \& Mattia, J. I. (1999). Axis I diagnostic comorbidity and borderline personality disorder. Comprehensive Psychiatry, 40, 245-252. doi: 10.1016/S0010-440X(99)90123-2 



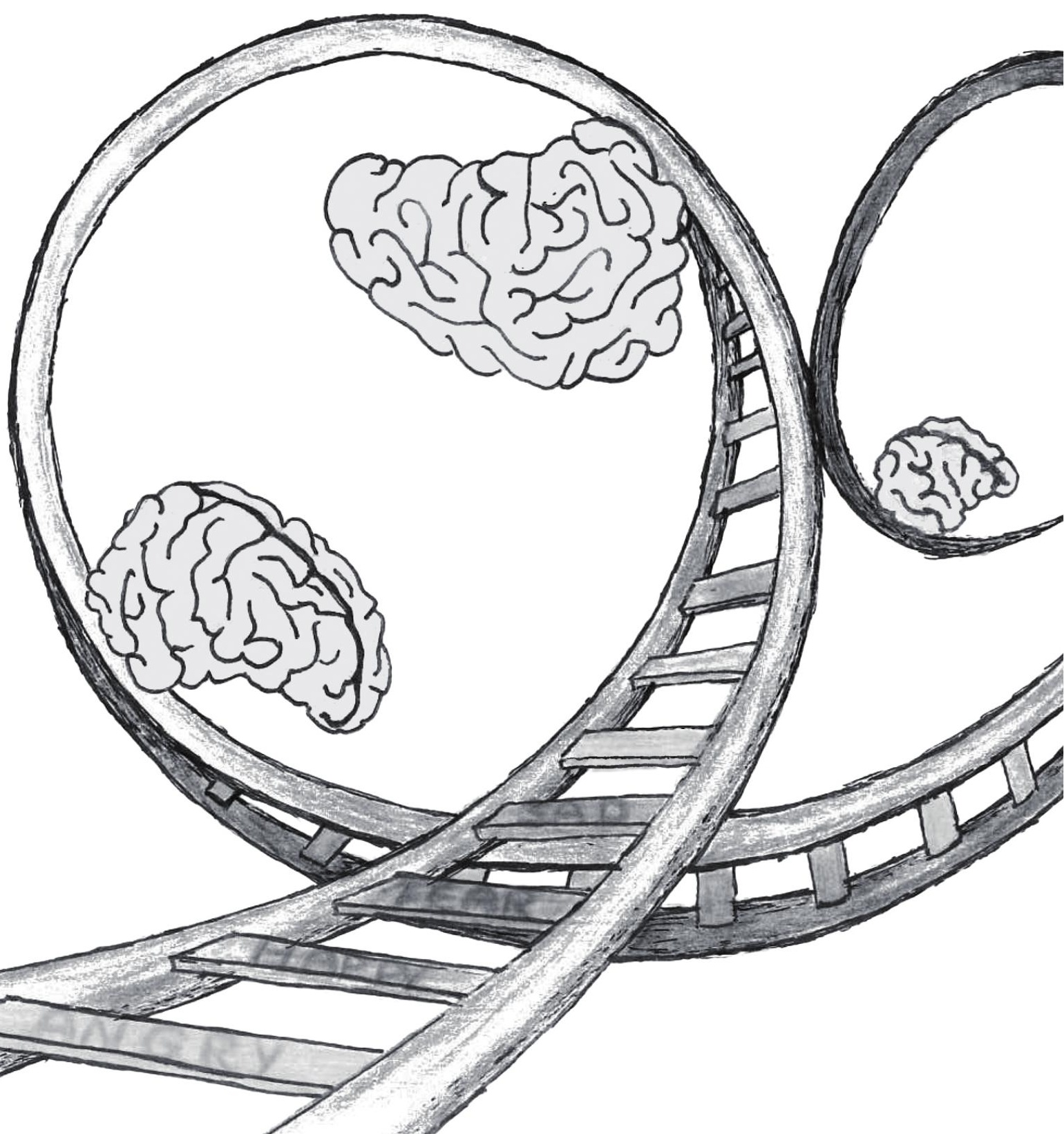




\section{Emotional sensitivity, emotion regulation and impulsivity in borderline personality disorder: A critical review of fMRI studies}

This chapter has been published as:

Van Zutphen, L., Siep, N., Jacob, G.A., Goebel, R. \& Arntz, A. (2015). Emotional sensitivity, emotion regulation and impulsivity in borderline personality disorder: A critical review of fMRI studies. Neuroscience and Biobehavioral Reviews, 51, 64-76. doi: 10.1016/j.neubiorev.2015.01.001. 


\section{Abstract}

Emotional sensitivity, emotion regulation and impulsivity are fundamental topics in research of borderline personality disorder (BPD). Studies using fMRI examining the neural correlates concerning these topics is growing and has just begun understanding the underlying neural correlates in BPD. However, there are strong similarities but also important differences in results of different studies. It is therefore important to know in more detail what these differences are and how we should interpret these. In present review a critical light is shed on the fMRI studies examining emotional sensitivity, emotion regulation and impulsivity in BPD patients. First an outline of the methodology and the results of the studies will be given. Thereafter important issues that remained unanswered and topics to improve future research are discussed. Future research should take into account the limited power of previous studies and focus more on BPD specificity with regard to time course responses, different regulation strategies, manipulation of self-regulation, medication use, a wider range of stimuli, gender effects and the inclusion of a clinical control group.

Keywords: Borderline personality disorder, fMRI, Neuroimaging, Emotional sensitivity, Emotion dysregulation, Emotional reactivity, Emotional responsivity, Impulsivity, Response inhibition 


\section{Introduction}

Borderline personality disorder (BPD) is a severe psychiatric disorder, which affects 1-3\% of the general population (Lenzenweger, Lane, Loranger \& Kessler, 2007; Trull, Jahng, Tomko, Wood \& Sher, 2010). The Diagnostic and Statistical Manual of Mental Disorders (DSM-V; American Psychiatric Association, 2013) defines the main features of BPD as a pervasive pattern of instability in interpersonal relationships, emotions, self-image as well as marked impulsive behavior. Furthermore, BPD patients are characterized by repeated self-injury, suicidal tendencies, reactive aggression and high rates of comorbid mental disorders (Leichsenring, Leibing, Kruse, New \& Leweke, 2011; Lieb, Zanarini, Schmahl, Linehan \& Bohus, 2004). BPD severely affects employment, and in combination with high use of the mental health care, results in high societal costs (van Asselt, Dirksen, Arntz \& Severens, 2007).

Leading theories on the etiology of BPD (Crowell, Beauchaine \& Linehan, 2009; Koenigsberg et al., 2009b; Linehan, 1993) propose that BPD is best understood as an emotion dysregulation disorder, caused by an increased emotional sensitivity and an inability to regulate emotional responses. The inability to regulate emotional responses might consequently result in marked impulsive behavior, characteristic for BPD patients. In the last decades researchers have used different behavioral and psychophysiological methods to test the hypothesized increased emotional sensitivity and diminished regulation (see for reviews Domes, Schulze \& Herpertz, 2009; Rosenthal et al., 2008). Results seem inconsistent. In line with the emotion dysregulation hypothesis, several studies show that BPD patients have a stronger emotional reaction to emotional stimuli when compared to non-patients (NPC), as indicated by an increased heart rate (Ebner-Priemer et al., 2007; Lobbestael \& Arntz, 2010), increased skin conductance responses (Lobbestael \& Arntz, 2010), larger startle response (Ebner-Priemer et al., 2005; Hazlett et al., 2007), decreased facial recognition accuracy (Bland, Williams, Scharer \& Manning, 2004; Levine, Marziali \& Hood, 1997) or a more accurate facial recognition (Lynch et al., 2006; Wagner \& Linehan, 1999). In contrast, studies also demonstrated that BPD patients had lower skin conductance responses (Herpertz, Kunert, Schwenger \& Sass, 1999) and lower levels of endocrine stress markers (Nater et al., 2010) when confronted with emotional stimuli or stressful situations, which seems to indicate lower levels of emotional reactivity. Behavioral data concerning impulsivity in BPD patients are also conflicting. Some studies did not report any differences between NPC and BPD patients during a go/no-go task (Jacob et al., 2010; Vollm et al., 2004), while others showed that BPD patients make significantly more commission errors, indicating that they are worse in response inhibition (Leyton et al., 2001; Mortensen, Rasmussen \& Håberg, 2010; Rentrop et al., 2008). Taken together, these studies show mixed findings concerning emotional reactivity as well as impulsivity in BPD.

In recent years an increasing number of neuroimaging studies have identified neural correlates associated with BPD (see for reviews Krause-Utz, Winter, Niedtfeld \& Schmahl, 
2014; Mauchnik \& Schmahl, 2010). Many of these studies (e.g. Koenigsberg et al., 2009b; Minzenberg, Fan, New, Tang \& Siever, 2007; Schulze et al., 2011; Silbersweig et al., 2007) highlighted a characteristic response in the frontolimbic brain network, comprised of an increased limbic activity and decreased prefrontal activity in BPD patients in response to emotional stimuli. As limbic brain areas have generally been associated with emotion detection and generation processes and prefrontal areas with cognitive control processes (Davidson \& Irwin, 1999; Phillips, Drevets, Rauch \& Lane, 2003), these findings have been interpreted as being in concordance with the emotional dysregulation hypothesis of BPD. Although there seems to be a concurrence of neural networks among the neuroimaging studies investigating emotional processing in BPD patients, like in behavioral studies also important differences have been observed. This raises questions about the conclusiveness of the results.

This article provides a critical review of fMRI studies investigating emotional sensitivity, emotion regulation and impulsivity in BPD patients. This review starts with a definition of the investigated constructs of emotional sensitivity, emotion regulation and impulsivity, followed by an overview of, to the best of the present authors' knowledge, all here-todate relevant publications. These publications are then discussed, summarizing important issues that remain unanswered and are suggested to be taken into consideration for future research. Included in this review are original published peer-reviewed articles, wherein authors make direct conclusions about emotional sensitivity, emotion regulation or impulsivity in BPD patients and compared the resulting brain activity to that of NPC. Given that in BPD research often negative stimuli are used, the present review will focus on $\mathrm{fMRI}$ studies using negative stimuli.

\section{Emotional sensitivity, emotion regulation and impulsivity: definitions}

Emotional sensitivity can be defined as a lower threshold to detect or respond to emotional stimuli, or a higher probability of experiencing stimuli as emotional (Carpenter \& Trull, 2013; Linehan, 1993). In BPD patients this might lead to hyperawareness to emotional stimuli and more frequent, intense and/or prolonged emotional experiences. Emotion regulation is a broad concept, referring to different methods and abilities that individuals use to influence the experience and expression of their emotions (Gross, 1998, 2007). These different methods can be categorized as either response-focused or antecedent-focused strategies (Gross, 1998, 2007). Response-focused strategies modulate emotional processes which occur late in the emotion generation process. This involves the inhibition of ongoing emotional expressive behavior such as facial expressions (bodily responses). Antecedentfocused strategies modulate emotional processes early in the emotion generation process, before the emotion response tendencies have become activated. The best-known examples of antecedent-focused strategies are attentional deployment (e.g. distraction) 
and cognitive reappraisal (e.g. distancing and reinterpretation). Cognitive reappraisal is an often studied emotion regulation strategy in BPD patients, which specifically refers to the ability to reinterpret a stimulus or situation (Gross, 1998, 2007).

Summarizing the set of brain areas involved in emotion generation and regulation is plagued by the fact that different authors use different, but also overlapping, sets of brain areas. In this review we follow the model of cognitive control of emotion (MCCE) as proposed by Ochsner et al. (2012). According to this model brain areas involved in emotion generation are the amygdala, ventral striatum, anterior insula and orbitofrontal cortex (OFC). Most frequently demonstrated brain areas showing an increased activity during emotion regulation of negative emotional stimuli are the dorsolateral prefrontal cortex (dIPFC), ventrolateral prefrontal cortex (VIPFC), dorsomedial prefrontal cortex (dmPFC) and anterior cingulate cortex (ACC) (Ochsner, Bunge, Gross \& Gabrieli, 2002; Ochsner et al., 2012; Phan et al., 2005). In accordance with the theory of emotional dysregulation in BPD, it is hypothesized that BPD patients show a greater activity in the aforementioned emotion generation brain areas and less activity in the emotion regulation brain areas during the application of emotion regulation strategies compared to NPC.

Impulsivity is a multidimensional concept consisting of a behavioral, cognitive and motivational dimension (Evenden, 1999; Moeller, Barratt, Dougherty, Schmitz \& Swann, 2001; Sebastian, Jacob, Lieb \& Tüscher, 2013a). The pattern of impulsivity often diagnosed in BPD patients is largely associated within the behavioral domain, including self-injury, reactive aggression, inappropriate anger, substance or alcohol abuse, unprotected sex, excessive spending of money and gambling, shoplifting, uncontrolled eating and reckless driving (American Psychiatric Association, 2013). Accordingly, in present review impulsivity is defined as a predisposition to rapidly and unplanned responses without considering the consequences (Moeller et al., 2001). Additionally, previous studies (Domes et al., 2006; Jacob et al., 2010; Sebastian et al., 2013a) state that impulsivity in BPD is especially evident under extreme emotional states or situations, this is specified as affective or emotional impulsivity. Key brain areas associated with impulsivity (response inhibition) in NPC are the inferior frontal cortex (IFG)/anterior insula, middle temporal gyrus, OFC/ventromedial prefrontal cortex (VmPFC), premotor areas, dIPFC, ACC and inferior parietal cortex (Garavan, Ross \& Stein, 1999; Horn, Dolan, Elliott, Deakin \& Woodruff, 2003; Sebastian et al., 2013b). In agreement with clinically shown marked impulsive behavior, it is hypothesized that BPD patients show less activity in these response inhibition areas compared to NPC. 


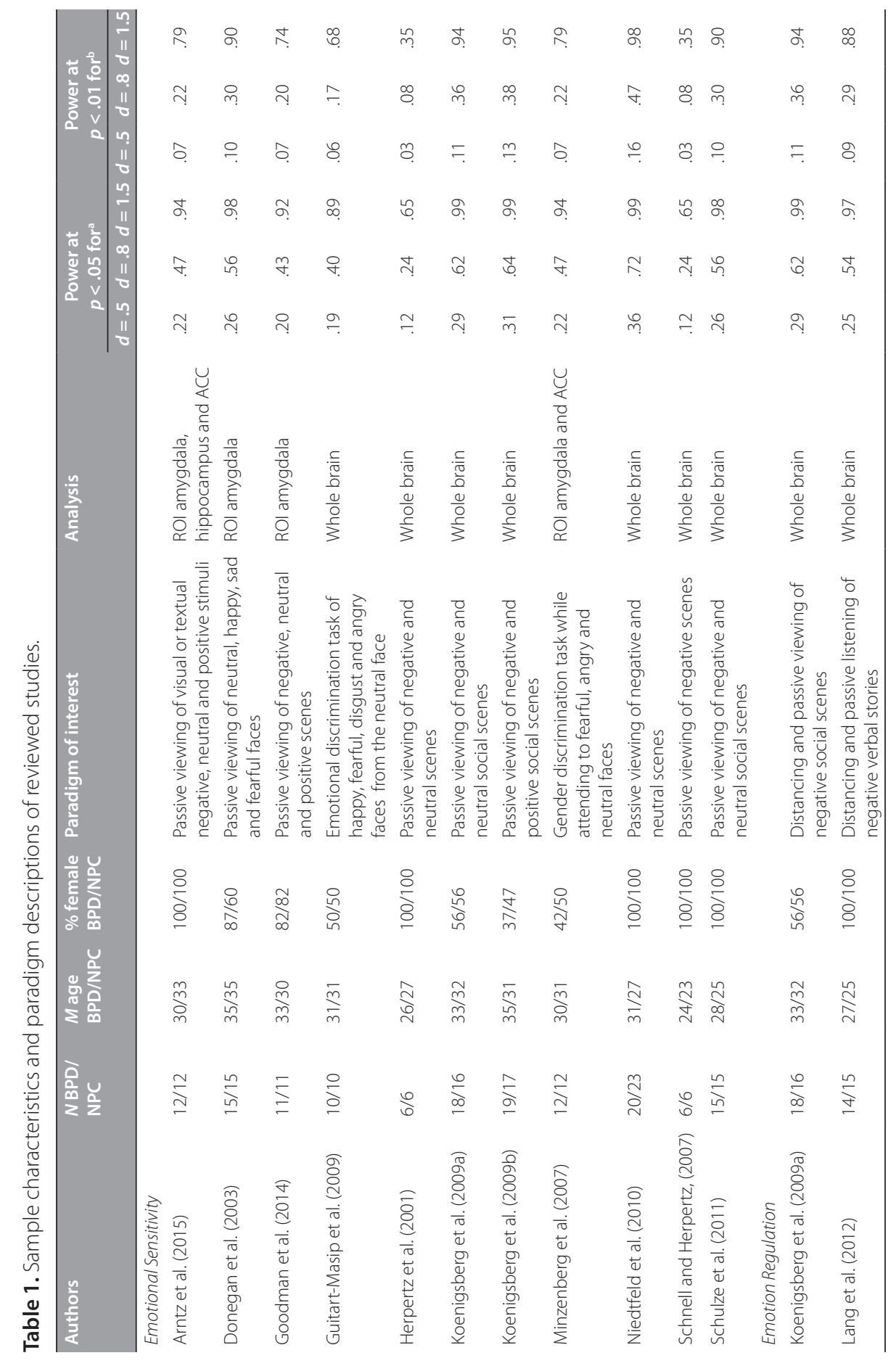




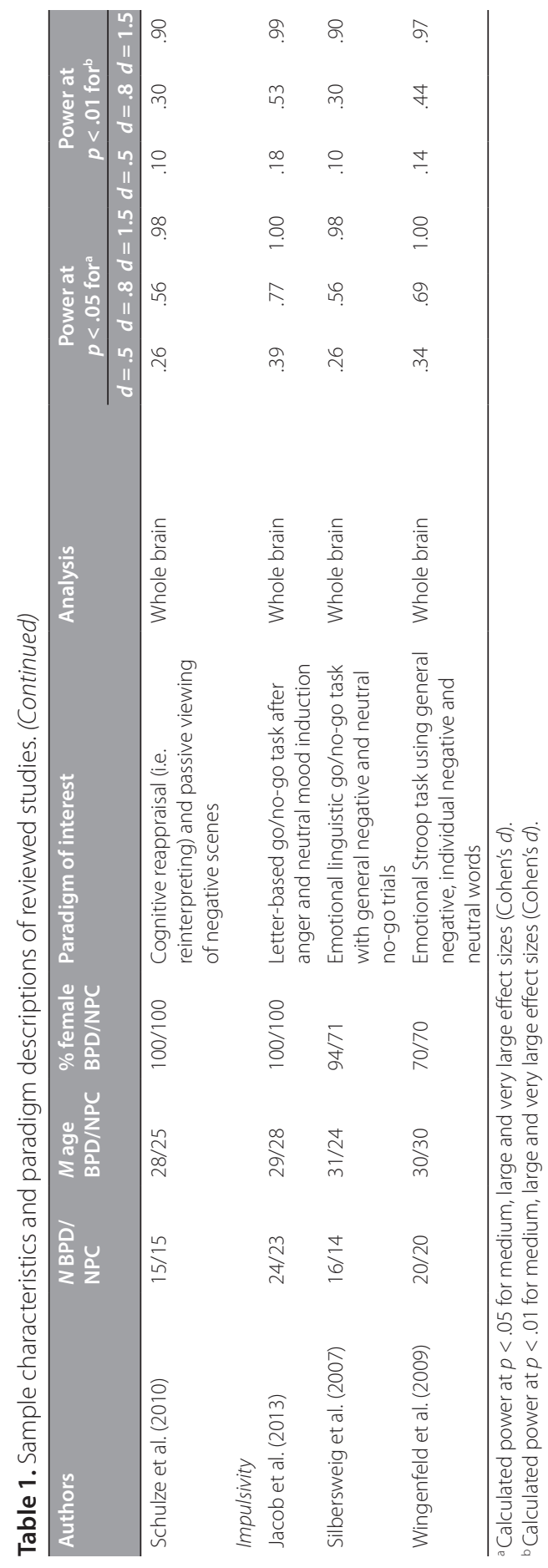




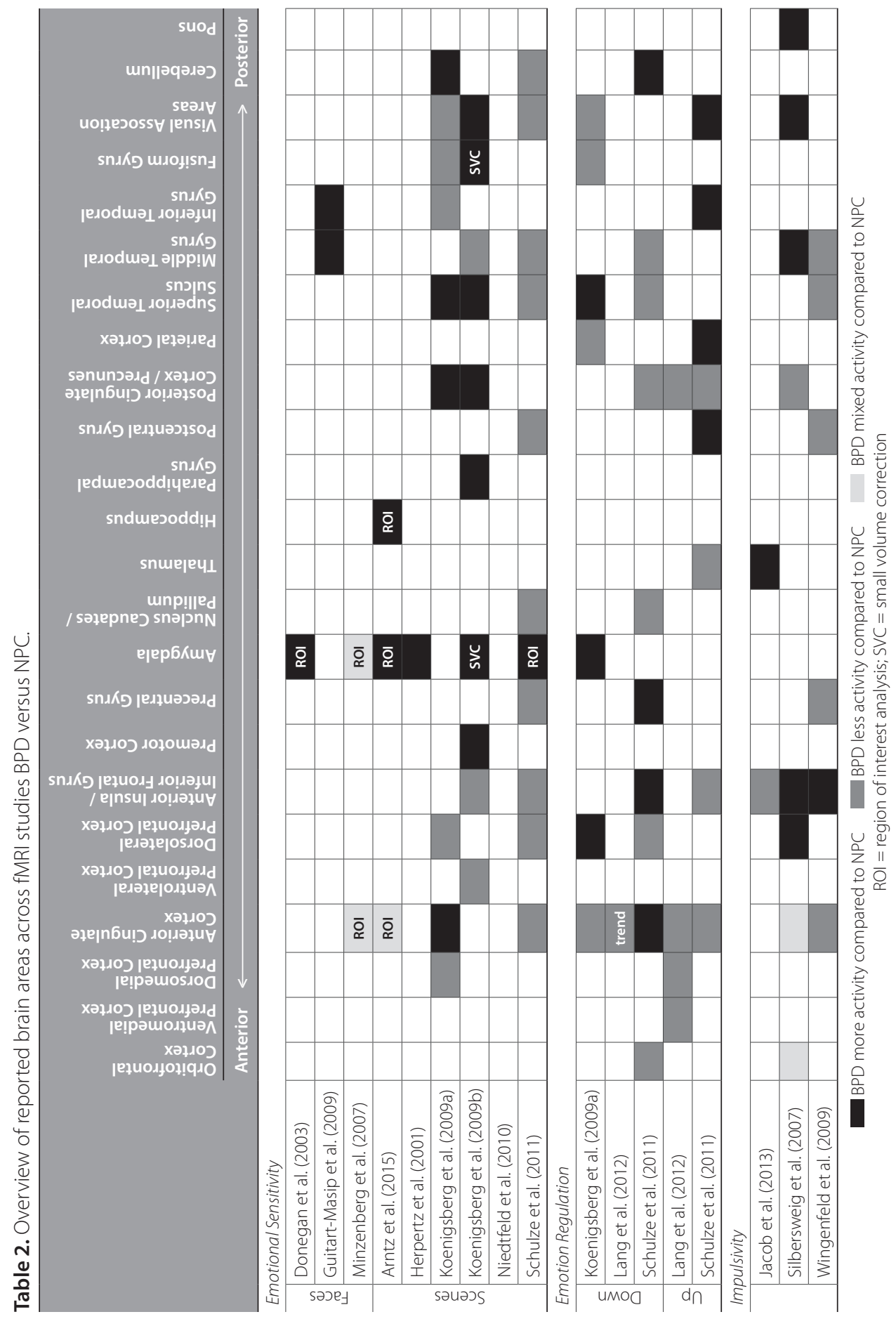




\section{Emotional sensitivity in BPD}

To examine emotional sensitivity in BPD patients, two different types of emotional stimuli have commonly been used in BPD fMRI studies so far. These two stimuli are facial expressions and emotional scenes. The first include the presentation of pictures of faces that vary in their emotional expression (e.g. happy, fearful, and angry) and the second presents pictures depicting scenes differing in their emotional content. Two studies used negative memories to examine emotional sensitivity, one of abandonment (Schmahl et al., 2003) and the other of childhood abuse (Schmahl, Vermetten, Elzinga \& Bremner, 2004). However, these are PET studies and therefore will be out of focus in current review. The results of the included studies will be discussed in the light of the model of the cognitive control of emotion. For an overview of all results see Table 2.

\section{Facial expressions}

To date, three studies have used stimuli depicting facial expressions to examine emotional sensitivity in BPD patients (Donegan et al., 2003; Guitart-Masip et al., 2009; Minzenberg et al., 2007; Table 1). All three studies used faces from the Ekman and Friesen set (1979), which contains 60 pictures of faces of ten different persons, six women and four men, displaying six different emotions: happy, sad, fearful, surprised, disgusted and angry. However, the facial expressions included, the task instructions and the analysis slightly varied across the three studies. In the study of Donegan et al. (2003) participants were instructed to attend to faces with a fearful, sad, happy or neutral expression. In the analyses, group differences in brain responses for these facial expressions were separately compared to the brain responses during the presentation of a fixation point. Minzenberg et al. (2007) instructed participants to identify the gender of neutral, fearful and angry faces that were presented. Brain responses to fearful and angry facial expressions were then compared to brain responses to neutral facial expressions. Finally, Guitart-Masip et al. (2009) used an emotional discrimination task, in which participants had to indicate which of two presented faces showed an emotional expression, or which of two lines was oriented vertical. Brain responses during the emotional discrimination trials of happy, fearful, disgust and angry facial expressions were then compared with brain responses during the orientation trials, using three contrasts: all emotional trials vs. orientation trials, positive discrimination (happy faces) vs. orientation trials and negative discrimination (fearful, disgust and angry faces) vs. orientation trials. To examine group differences two of the three studies (Donegan et al., 2003; Minzenberg et al., 2007) conducted a priori region of interest (ROI) analyses on the amygdala, the other study (Guitart-Masip et al., 2009) conducted a whole brain analysis and a post hoc ROI analyses on the amygdala. Moreover, Minzenberg et al. (2007) performed an ACC ROI analysis. 
The results of two studies (Donegan et al., 2003; Minzenberg et al., 2007) confirmed the hypothesis of increased emotional sensitivity, showing greater amygdala activity in BPD patients compared to NPC during the presentation of emotional facial expressions; Donegan et al. (2003) showed greater amygdala activity for the BPD patients during the presentation of all three emotional facial expressions (happy, sad and fearful) and even during the presentation of the neutral faces. Interestingly, Minzenberg et al. (2007) found a greater amygdala and lower ACC activity only during the presentation of fearful faces. This amygdala and ACC pattern was reversed for angry faces. Using the emotion discrimination paradigm, Guitart-Masip et al. (2009) could not replicate these differences in amygdala and ACC activity between the BPD and NPC group (Table 2).

At a $p$-value of .05 these three studies had an estimated power below $80 \%$ to correctly reject the null hypothesis at a medium $(d=0.5)$ or large $(d=0.8)$ effect size (Table 1$)$. To detect a very large effect size $(d=1.5)$ the power was sufficient (above $80 \%)$ for these three studies. Regarding a more strict $p$-value of .01 only Donegan et al. (2003) had a sufficient power of $90 \%$ to detect a very large effect size, Minzenberg et al. (2007) was close to having sufficient power (79\%) of correctly rejecting the null hypothesis at a very large effect size (Table 1).

\section{Emotional scenes}

So far, eight studies have used emotional scenes to study emotional sensitivity in BPD patients (Table 1). The first fMRI study using this approach (Herpertz et al., 2001) used intermixed social and non-social scenes, six later studies used social emotional scenes only (Goodman et al., 2014; Koenigsberg et al., 2009a; Koenigsberg et al., 2009b; Niedtfeld et al., 2010; Schulze et al., 2011) and one study used visual emotional scenes and textual emotional stimuli (Arntz et al., submitted). All studies used pictures selected from a standardized database (e.g. IAPS; Lang, Bradley \& Cuthbert, 1997). In these studies participants were either instructed to attend to the pictures (Goodman et al., 2014; Herpertz et al., 2001; Schnell \& Herpertz, 2007) or to look at the pictures and allow themselves to respond naturally (Arntz et al., submitted; Koenigsberg et al., 2009a; Koenigsberg et al., 2009b; Schulze et al., 2011). To examine the emotional sensitivity of the BPD patients, two contrasts were used among the studies: 1) brain responses during looking at negative scenes were compared with looking at neutral scenes (Arntz et al., submitted; Herpertz et al., 2001; Koenigsberg et al., 2009a; Niedtfeld et al., 2001; Schulze et al., 2011), or 2) brain response during looking at negative scenes were compared to a fixation condition (Koenigsberg et al., 2009b). Schnell and Herpertz (2007) and Goodman et al. (2014) did not compare negative scenes with either neutral scenes or to a fixation condition and therefore will not be further discussed in present review.

When comparing brain activity during the negative scenes to activity during the neutral scenes or fixation, BPD patients compared to NPC showed enhanced activity of 
the amygdala (Arntz et al., submitted; Herpertz et al., 2001; Koenigsberg et al., 2009b; Schulze et al., 2011) and diminished activity of the anterior insula (Koenigsberg et al., 2009b; Schulze et al., 2011). Interestingly also brain areas involved in emotion regulation are found across studies examining emotional sensitivity in BPD patients, including the ACC, vIPFC, dIPFC and dmPFC (Table 2). Using the contrast negative vs. neutral, Koenigsberg et al. (2009a) displayed an enhanced activity of the ACC in BPD patients compared to NPC, while Schulze et al. (2011) for the same contrast showed less activity in BPD patients. Arntz et al. (submitted) showed a weaker left and a stronger right ACC activity to negative vs. neutral emotional scenes for BPD patients compared to NPC. Furthermore, Koenigsberg et al. (2009b), Koenigsberg et al. (2009a) and Schulze et al. (2011) found a decreased activity in the VIPFC, dIPFC and dmPFC in BPD patients compared to NPC. Niedtfeld et al. (2010) showed a similar activity for negative stimuli as for neutral stimuli and therefore revealed no group differences of brain responses during negative vs. neutral scenes.

The estimated power of these studies at a $p$-value of .05, for a medium effect size, ranges between 12 and 36\% to correctly reject the null hypotheses, and between 24 and 72\% for a large effect size (Table 1). The power to detect a very large effect size was sufficient for most studies with the exception of the study with a very small sample size of only six participants in each group (Herpertz et al., 2001). At a p-value of .01 none of the studies had a power above $80 \%$ to correctly reject the null hypothesis at a medium or large effect size (Table 1). Again Herpertz et al. (2001) had insufficient power to detect a very large effect size, while Arntz et al. (submitted) had almost sufficient power (79\%) to detect a very large effect size. The four other studies had a power between 90 and 98\% to correctly reject the null hypothesis at a very large effect size (Table 1).

In conclusion, the most consistent finding in fMRI emotional sensitivity studies (7 out of 10 studies) is the greater amygdala activity in BPD patients during passive viewing of facial expressions as well as IAPS pictures, compared to NPC. In addition, BPD patients show an altered activity in an extended neural network, including the hippocampus, parahippocampal gyrus, anterior insula, ACC, vIPFC, dIPFC, dmPFC, superior temporal sulcus (STS) and posterior cingulate cortex (PCC)/precuneus. However, in particular areas some studies showed a decreased activity while other studies show an increased activity.

\section{Emotion regulation in BPD}

Although an important part of the emotion dysregulation theory focuses on impaired emotion regulation skills in BPD patients, to our knowledge only three published fMRI studies (Koenigsberg et al., 2009a; Lang et al., 2012; Schulze et al., 2011; Table 1) have examined this specific hypothesis. The results of these studies will be discussed with regard to previously reported brain areas involved in the model of the cognitive control of emotion. All results are shown in Table 2. 
Across the studies, BPD patients and NPC are instructed to apply different regulation strategies to modulate their emotional responses to presented stimuli. These stimuli were either negative emotional scenes (Koenigsberg et al., 2009a; Schulze et al., 2011) or negative verbal stories (Lang et al., 2012). With regard to the instructions, in the studies by Koenigsberg et al. (2009a) and Lang et al. (2012) participants were told to distance themselves from the stimuli by viewing or listening to the stimuli from a detached third-person perspective. Schulze et al. (2011) instructed their participants slightly different: participants had to imagine that the situation was not real or that they were a detached observer. To examine the effect of the emotion regulation instruction, brain responses during this condition were compared to brain responses during a look condition (experience the naturally evoked emotional state). In addition, Schulze et al. (2011) and Lang et al. (2012) explored the effects of up-regulation (increase). Therefore, an up-regulation minus look contrast was applied. During up-regulation participants were instructed to imagine that they, or a close relative, were involved in the situation (Schulze et al., 2011) or imagine themselves as the central figure of the story and heighten their feelings of closeness (Lang et al., 2012).

Result showed that application of the emotional down-regulation techniques during the presentation of the negative stimuli, relative to the passive viewing/listening condition of negative stimuli, led to greater activity in emotion generation areas in BPD patients compared to NPC, including the amygdala (Koenigsberg et al., 2009a) and anterior insula (Schulze et al., 2011), whereas activity in the OFC was diminished (Schulze et al., 2011). With regard to brain areas involved in regulation processes, Koenigsberg et al. (2009a) showed in BPD patients compared to NPC less activity in the ACC and greater activity in the dIPFC, whereas Schulze et al. (2011) reported the opposite pattern for both brain areas. In line with Koenigsberg et al. (2009a), Lang et al. (2012) reported a trend toward a diminished activity in the ACC for the BPD patients.

During the up-regulation condition of negative stimuli, relative to the passive viewing/ listening condition of negative stimuli, Schulze et al. (2011) found that BPD patients compared to NPC, showed a decreased activity in the ACC and anterior insula. Since they did not find an increased activity in emotional generation and regulatory brain areas during up-regulation, they stated that emotional dysregulation in BPD patients might be exclusive to down-regulation. In contrast to these results, Lang et al. (2012) did find less activity in regulation associated brain areas such as the VmPFC, dmPFC and ACC, in BPD patients compared to NPC. They could not conclude that emotional dysregulation in BPD patients was exclusive to down-regulation.

At a $p$-value of .05 and .01 these studies were able with an estimated power above $80 \%$ to correctly reject the null hypothesis at a very large effect size (Table 1). For both significance levels the power was below $80 \%$ to detect a medium or large effect size.

In sum, the most consistent finding in $\mathrm{fMRl}$ emotion regulation studies (4 out of 5 studies) 
is the decreased ACC activity in BPD patients compared to NPC during down-regulation as well as during up-regulation. Results across the medial and dorsolateral prefrontal activity during both regulation conditions of negative stimuli were variable. In addition, 2 out of 3 studies indicate a difficulty to modulate the activity of the amygdala and anterior insula shown by an increased activity of these areas during down-regulation. Together conclusions concerning the support of the conceptualization of emotional dysregulation in BPD patients for negative social stimuli should be drawn with caution. The number of studies is still limited and the results seem variable across the studies. Besides, especially beyond the emotional regulatory network, there seems to be a major variation of reported brain areas and some studies even report a complete opposite pattern of brain activity compared to other studies.

\section{Impulsivity in BPD}

To date, three fMRI studies have examined impulsivity in BPD, they used either a go/no-go task (Jacob et al., 2013; Silbersweig et al., 2007) or an affective Stroop task (Wingenfeld et al., 2009; Table 1). The results of these studies will be discussed with respect to earlier reported brain areas involved in impulsivity. An overview of all results is given in Table 2 .

Concerning both studies which used the go/no-go task, the task design and instructions differed. Silbersweig et al. (2007) used an affective go/no-go task in which participants were instructed to press a button immediately after reading a word. The words used differed in their emotional valence and appeared in normal or italic font. Participants were instructed to inhibit their response after a word in italics. Differences in impulsivity were examined by contrasting negative no-go word trials with neutral no-go word trials (negative no-go trials). Jacob et al. (2013) used a letter-based go/no-go task in which participants had to press a button corresponding to all letters except the letter "X", after the induction of an angry, joyful or neutral mood. To examine differences in impulsivity the no-go trials after the anger induction were contrasted with the no-go trials after neutral induction (anger no-go trials). Finally, in the Stroop task by Wingenfeld et al. (2009) the participants were instructed to name the color of the presented words as fast and accurate as possible. Each color was represented by a respective colored button on a button box. In this task neutral, general negative and individual negative words were used. Brain activity during general and individual words was compared with brain activity during neutral words to examine differences in impulsivity.

The results of the go/no-go task of Silbersweig et al. (2007) showed during the negative no-go trials a greater activity in the lateral OFC, anterior insula and ACC, and less activity in the medial OFC and subgenual ACC in BPD patients, compared to NPC. Additionally, the [no-go minus go] $\times$ [negative minus neutral] interaction showed a greater activity in the dIPFC in BPD patients compared to NPC. Jacob et al. (2013) reported in BPD patients 
compared to NPC, a decreased activity in the inferior frontal cortex during the anger nogo trials. The results of the Stroop task showed no significant differences between the BPD patients and NPC in brain areas associated with response inhibition during the presentation of the general negative minus the neutral trails (Wingenfeld et al., 2009). For the comparison of individual negative words a decreased ACC activity was shown in BPD patients. A different pattern of brain activity was shown between the go/no-go task and the Stroop task. The Stroop task was less associated with response inhibition related brain areas and a decreased ACC activity, while the go/no-go task was more associated with response inhibition related areas and an increased ACC activity.

At a $p$-value of .05 and .01 these studies had an estimated power above $80 \%$ to correctly reject the null hypothesis at a very large effect size (Table 1). For both significance levels the power was insufficient (below 80\%) to correctly reject the null hypothesis at a medium or large effect size.

Taken together, conflicting results of brain areas involved in inhibition are shown by the impulsivity studies when negative emotions are evoked in BPD patients. However, the fMRI studies examining impulsivity in BPD patients comprise quite a diversity of task designs, which might explain the variety of brain areas reported across the studies. From this data it seems too early to draw conclusions concerning the hypothesis that BPD patients have difficulty in modulating their impulses in the setting of a negative state.

\section{Discussion}

The present review provides an overview of fMRI studies examining the neural correlates of emotional sensitivity, emotion regulation and impulsivity in BPD patients in response to negative stimuli. Regarding emotional sensitivity in BPD patients, in line with the hypothesis an enhanced activity of the amygdala was shown to be most consistent across the studies. The hypothesis that emotional dysregulation in BPD patients would be manifested from neural correlates of increased limbic areas (e.g. amygdala) and decreased regulatory areas (e.g. vIPFC, dIPFC, ACC) was partly supported. A consistent decreased activity of the ACC was shown, while the medial and dorsolateral prefrontal areas showed variable activity. Evidence of a failure (lack of activation) of inhibitory areas (e.g. IFG, vmPFC, dIPFC, ACC) possibly underlying impulsivity in BPD patients is inconclusive. The overall findings are more inconclusive as expected, and open new avenues for future research.

First it should be noted that the studies reviewed have low statistical power, even at lenient (uncorrected) $p$-levels of .05 and .01, which limits the likelihood of finding a true effect. These studies could only detect very large effect sizes. The average statistical power used in different subfields of cognitive neuroimaging is already low, between 8 and $31 \%$, and it is with the scientific developments nowadays more important to investigate subtle effects 
than before (Button et al., 2013). In order to increase the power and detect smaller effects one should increase the sample size. Another approach is to try to increase the effect sizes by improving the signal/noise ratio by methodological and technical means. For example, scanning parameters can be optimized to reduce signal drop out and image distortions (Weiskopf, Hutton, Josephs \& Deichmann, 2006). Dependent on the brain area of interest, one can adjust the slice tilt, direction of the phase-encoding and z-shimming moment to optimize BOLD sensitivity. In addition, while it is assumed that there is a linear relationship between the neural activity and hemodynamic response, equal for all participants, it is well established that this relationship is not always linear and varies across participants. Therefore, nonlinear temporal statistical models might sometimes be more accurate in estimating the temporal relationship between the neural activity and hemodynamic response and could increase imaging power (Liu, Frank, Wong \& Buxton, 2001). Finally, taking into account anatomical variability, by means of refining brain normalization or performing a surface based alignment technique, could also increase statistical power (Frost \& Goebel, 2012). For instance, cortex based alignment uses detailed individual curvature information to align the brains of the individual participants to a group average. In conclusion, aforementioned recommendations can be applied in order to reach the necessary increase of imaging power to detect smaller effects.

Additionally, a number of other methodological issues could explain the inconsistencies such as different implementation of emotion regulation strategies, use of medication and choice of neutral stimuli. Also different measurement and analyzing methods concerning the amygdala could explain some inconsistencies concerning the involvement of the amygdala. Topics to consider improving future research are manipulation of self-regulation, a more extensive range of stimuli, gender differences and the use of a clinical control group. We will now discuss these issues in more detail.

\section{Inconsistencies concerning the involvement of the amygdala in BPD}

Early studies of the amygdala revealed that the amygdala plays a critical role in the processing of fear (Whalen, 1998). Nowadays it is suggested that the amygdala plays a broader role in emotion related processes for a wide range of stimuli (e.g. negative, positive and social; Adolphs \& Spezio, 2006; Phan, Wager, Taylor \& Liberzon, 2002). Therefore, recently more general approaches assume that the amygdala is not specifically sensitive to emotional valence of stimuli, but more associated with salience, novelty or arousal. Furthermore, the amygdala is extensively connected with cortical and subcortical structures. Because of its broad connectivity, the amygdala influences cognitive interactions in response to emotional stimuli such as emotional or reward learning, memory enhancement, attention modulation, decision making and regulation (e.g. Bechara, Damasio \& Damasio, 2003; Pessoa, 2011; Phelps, 2006). 
It is interesting that while the emotional dysregulation theory suggests an association with a hyperreactivity of the amygdala, some studies did not find this increased amygdala response (Guitart-Masip et al., 2009; Koenigsberg et al., 2009b), while others showed an amygdala response after a $\mathrm{ROI}$ analysis or a small volume correction (Koenigsberg et al., 2009a; Schulze et al., 2011). These inconsistent findings can be explained in three-fold. First, it can be difficult to detect amygdala responses. This could be due to its localization next to air-filled spaces leading to susceptible artifacts that result in signal dropouts and image distortions (Morawetz et al., 2008). Therefore, to get more insight in the involvement of the amygdala, it would be advised to optimize scanning acquisition parameters. A recent study investigated rather simple modifications at the acquisition level to reduce susceptibility artifacts of the amygdala (Morawetz et al., 2008). It is suggested that a slice thickness of $2 \mathrm{~mm}$, slice orientation along the AC-PC plane, $36 \mathrm{~mm}$ echo time and $4 \mathrm{~mm}$ spatial filter result in the least artifacts. Additionally, because of the massive corrections for multiple testing in $\mathrm{FMRI}$ data it is difficult to detect weak differential activation levels of the amygdala in a whole brain analysis. To reduce the multiple comparisons problem caused by the large number of voxels, ROI analyses can be performed (Poldrack, 2007). Since the response profile of the amygdala across hypothesis-relevant conditions forms an important basis of testing theories about emotional sensitivity and emotion regulation in BPD, it is suggested to perform a $\mathrm{ROI}$ analysis of this area (in combination with increased sample size, see above) in order to enhance detection sensitivity. This will also substantiate interpretations in case a lack of activation or a lack of group differences is found. The same argument holds for adjustment of the statistical threshold, one could consider exploring whether group differences of the amygdala are present at the most liberal $p$-value of .05 (uncorrected), to prevent type-Il errors.

Second, the possibility of amygdala habituation might be present when repeatedly presenting similar emotional stimuli. Previous studies in NPC showed that the amygdala response rapidly declines after repeatedly presented facial stimuli (Breiter et al., 1996) and visual scenes (Fischer, Furmark, Wik \& Fredrikson, 2000). The only study which showed a group difference in the amygdala after a whole brain analysis was the study of Herpertz et al. (2001). Interestingly, they measured emotional sensitivity during one single run, while other studies acquired images throughout more runs presenting stimuli repeatedly (Koenigsberg et al., 2009a; Koenigsberg et al., 2009b; Niedtfeld et al., 2010; Schulze et al., 2011). This might be suggestive evidence for a habituation effect of the amygdala. Consequently it would be worthwhile to take into account this possible habituation effect by means of including time as a variable in the analysis. This is especially interesting since the theory of Linehan makes a statement about delayed habituation effects concerning emotional responses in BPD patients. It is suggested that BPD patients are characterized by a prolonged emotional experience due to a slow return to baseline arousal compared to NPC. Two recent fMRI 
studies showed a lack of decrease of amygdala activity with time after repeatedly presented pictures in BPD patients compared to NPC (Hazlett et al., 2012; Kamphausen et al., 2013). This delayed habituation accounted for the hyperactivity of the amygdala and could be interpreted as evidence for the prolonged duration of emotional responses in BPD patients. Of note, habituation effects could also exhibit in various other brain areas implicated in emotion processing such as the ACC, medial PFC and dIPFC (Phan, Liberzon, Welsh, Britton \& Taylor, 2003; Wright et al., 2001). Studying habituation will help to identify a core neural network of emotional sensitivity in BPD patients.

Third, individual differences in dissociation might explain conflicting findings across the studies. BPD is associated with the presence of dissociative symptoms (American Psychiatric Association, 2013; Zanarini, Ruser, Frankenburg \& Hennen, 2000). According to the frontolimbic disconnection model of dissociation, the medial PFC inhibits the amygdala and dampens the emotional reactivity and the autonomic response (Sierra \& Berrios, 1998). A study in BPD patients showed that patients with low dissociation showed a larger startle response compared to patients with high dissociation (Ebner-Priemer et al., 2005). This suggests that the moderating effect of dissociation should be taken into account.

In sum, due to the importance of the amygdala for testing the hypothesis, scanning parameters to detect the amygdala should be optimized and (exploratory) ROI analysis or adjustment of the statistical threshold should be considered. Additionally, habituation of amygdala responses should be investigated explicitly, especially with regard to the hypothesis of a delayed habituation in BPD patients. Finally, dissociation differences should be taken into account.

\section{Beyond the amygdala}

When the emotional dysregulation theory of BPD was first proposed (Linehan, 1993) the main focus of fMRI studies was on the amygdala. However, considering the findings of the present review one might propose that the neural network involved in BPD entails more than a hyperactivity of the amygdala (Table 2). BPD patients do not only show enhanced activity in brain areas associated with emotional generation and salience (i.e. amygdala; Adolphs \& Spezio, 2006; Phillips et al., 2003), but also in important other brain areas, including the anterior insula, dIPFC, ACC, PCC/precuneus and STS. Other fMRI paradigms indicate that besides affective processing the anterior insula is also involved in self-awareness and empathy (Craig, 2009; Singer, Critchley \& Preuschoff, 2009), the PCC/precuneus in selfreferential processing (Cavanna \& Trimble, 2006; Vogt, Vogt \& Laureys, 2006), and the STS in mentalizing (Allison, Puce \& McCarthy, 2000; Saxe, Xiao, Kovacs, Perrett \& Kanwisher, 2004). These functions are often related to social information processing (Adolphs, 2001; Olsson \& Ochsner, 2008). Besides emotional instability, BPD is also characterized by difficulties of social information processing such as interpersonal relationships, social attachment and social 
rejection (American Psychiatric Association, 2013; Lazarus, Cheavens, Festa \& Rosenthal, 2014; Roepke, Vater, Preissler, Heekeren \& Dziobek, 2012). Therefore responses in the anterior insula, PCC/precuneus and STS might be confounded by an underlying impairment in social information processing in BPD patients. Consequently, next to an emotional social condition it might be useful to manipulate social versus non-social contents by means of including an emotional non-social condition, such as disgust, to examine which brain activity in BPD patients can be explained by difficulties in non-social emotional information processing and which can be explained by difficulties in social emotional information processing.

\section{Emotion regulation strategies}

It is suggested that BPD patients have problems to regulate their emotional responses due to failure to activate regulatory brain areas (e.g. vIPFC, dIPFC, ACC; Krause-Utz et al., 2014). An issue to pinpoint is that across the present studies in BPD patients only cognitive reappraisal strategies were investigated and it has not been examined whether certain emotion regulation strategies might be more efficient than others for BPD patients. Schulze et al. (2011) specifically state to have used a reappraisal strategy, namely reinterpretation, however the instructions used in this study could have resulted in a combination of reinterpretation and distancing. This is an important distinction to make, as Koenigsberg et al. (2009a) suggest that distancing might be a better strategy compared to reinterpreting for BPD patients since they have particular difficulty in interpersonal social situations. Therefore, it would be important to discriminate by means of a direct comparison between both reappraisal strategies reinterpretation and distancing in BPD patients which strategy is superior. Besides, it can also be helpful to extend research beyond the current applied reappraisal strategies for regulation. Another regulation strategy of interest could be distraction. Studies in NPC show that both reappraisal as well as distraction are successful in regulating emotional responses and in decreasing the activity of the amygdala, but a stronger reduction was shown for distraction (Kanske, Heissler, Schonfelder, Bongers \& Wessa, 2011; McRae et al., 2010). This might be in line with the assumption that in distraction one should focus the attention away from the emotion content of the stimulus, while in reappraisal one should focus on the emotion content of the stimulus in order to re-evaluate the stimulus. Both strategies showed an increased activation of the prefrontal and cingulate areas for cognitive control, in reappraisal associated with emotion processing and in distraction associated with selective attention. Taken this into account distraction could, next to reappraisal, also be an effective regulation strategy for BPD patients. Unlike reappraisal and distraction, suppression occurs late in the emotions generation process (i.e. response-focused) and requires more effort to manage emotional responses. Therefore, suppression is associated with poorer psychological health and considered to be an unhealthy regulation strategy (John \& Gross, 2004). It might be that BPD patients mainly 
use suppression as regulation strategy. However, this is difficult to examine with current regulation paradigms because BPD patients are instructed to apply reappraisal techniques. Consequently it should be examined which regulation strategy BPD patients use, and if they use suppression how this might differ from instructed regulation strategies such as reappraisal and distraction.

Additionally, research in NPC has demonstrated that with regard to timing the PFC shows an early response during cognitive reappraisal (0-4.5 s; Goldin, McRae, Ramel \& Gross, 2008). In accordance with these results, another study in BPD patients compared to NPC also showed brain activity particularly in the early interval in both groups (Lang et al., 2012). Nevertheless, in order to regulate their emotional responses BPD patients failed to activate the prefrontal brain areas (e.g. dIPFC, dmPFC, ACC) in this early phase. This suggests that optimal cognitive strategies are those that decrease limbic activity early in the emotion generation process.

Another matter to consider in future research might be the difficulty to verify how and if participants performed the regulation strategies the way they were instructed. Most of the studies relied on self-report measures, but did not include objective measures like eye-gaze to monitor performances of the participants within the scanner. Results of a study in NPC showed that during the regulation condition participants fixated shorter at the emotional parts of the image compared to the attend condition (van Reekum et al., 2007). These gaze fixations predicted changes in the blood-oxygen-level dependent (BOLD) signal change in brain areas associated with the regulation condition. So since looking away or closing their eyes could intervene with regulation responses monitoring eye-gaze is an interesting measure to control for this possible interference.

Taken together, to examine whether BPD patients differ in the neural correlates of different strategies of emotion regulation, more research should be done to, direct comparisons of various emotion regulation strategies, whilst assessing to what degree participants actually use the intended strategy. Furthermore, one should investigate which emotion regulation strategy is most effective for BPD patients.

\section{Manipulation of self-regulation}

BPD patients are often confronted with social-emotional triggers or situations in which they have difficulty regulating their emotions or inhibiting their impulsive responses. One could therefore hypothesize that the demands of the kind of studies discussed in the present review are high for BPD patients and therefore they might become exhausted. The strength model of self-regulation (limited-resources model) of Baumeister theorized that selfregulation relies upon limited resources, this is also referred to as ego depletion (Baumeister \& Heatherton, 1996). It is common that people try to bring themselves out a negative emotion, therefore we use our resources to intervene in ongoing emotions and regulate 
our negative emotions. In this view negative emotions might predispose attempts at selfregulation. Continuously regulating might, according to this model, result in 'depletion' of self-regulation resources. A recent study in NPC demonstrated that compared to the control group, participants who engaged in effortful self-regulation showed an increased activity of the amygdala and reduced functional connectivity between the amygdala and VmPFC for negative scenes (Wagner \& Heatherton, 2013). This might indicate that after effortful selfregulation a failure in top-down control of the amygdala occurs, which could result in an increased difficulty of regulating emotions or inhibiting impulsivity.

Recently research has begun to explore countermeasures to overcome ego depletion; a novel stimulus, a change of context or new motivation can bring about a return to previous levels of functioning, questioning the limited-resources model (Martijn, Alberts \& de Vries, 2006). This might suggest that a depleted state does not reflect complete exhaustion but a temporary deficit. Therefore, it could also be that the phenomena attributed to depletion in earlier studies are difficulties in maintaining sustained attention. In BPD patients this might be caused by a competition for priority of unpleasant emotions or intrusions. In the future experimental manipulation of specific self-regulation strategies in the social and/or emotional domain should be examined in BPD patients to further shed light on what selfregulation processes are compromised in BPD.

\section{Use of psychotropic medication}

Use of psychotropic medication is very common in BPD. About 75\% of the BPD patients are regularly taking psychotropic medication, and these rates remain relatively stable after six years follow-up (Zanarini, Frankenburg, Hennen \& Silk, 2004). It is assumed that different classes of psychotropic medication target specific symptoms of BPD; antidepressants and mood stabilizers are often used to target affective dysregulation and impulsive-behavioral dyscontrol, and antipsychotics for cognitive-perceptual symptoms (Lieb et al., 2004). Following these statistics, the majority of the BPD fMRI studies seem unrepresentative, as 10 out of the 15 reviewed studies excluded BPD patients currently using medication. However, research indicates that use of medication is an important confounding factor when studying brain activity. For example, it has been shown that psychotropic medication (e.g. SSRIs), compared to placebo, dampens amygdala activity in response to fearful facial expressions (Anderson et al., 2007; Arce, Simmons, Lovero, Stein \& Paulus, 2008; Murphy, Norbury, O'Sullivan, Cowen \& Harmer, 2009). Aware of these findings, many researchers are hesitant to include patients on medication and often require patients to be free of medication several weeks prior to scanning, ranging from 2 (Koenigsberg et al., 2009a; Koenigsberg et al., 2009b; Niedtfeld et al., 2010; Schulze et al., 2011) till 8 weeks (Guitart-Masip et al., 2009; Lang et al., 2012). However, for some medication the washed out effect could be several months and even if the medication is washed out there still can be lasting effects in the 
brain such as structural, connectivity or compensatory changes (Hafeman, Chang, Garrett, Sanders \& Phillips, 2012). Furthermore, requiring patients to stop their medication seems ethically problematic and is likely to contribute to some form of sampling bias.

An often observed approach to the restriction of medication use, is the application of post hoc statistical analyses in which medicated versus non-medicated participants are compared to replicate the changes in brain areas in both groups. A limitation of this approach is that these analyses often lack the power to reliably assess the influences of medication on brain activity. As a solution to this problem it is suggested that researchers should examine correlations between medication load (i.e. number and dose) and changes in brain activity, which should have more variance and there might be enough variance to add as a regressor in the analysis (Hafeman et al., 2012). Another problem with such approaches is that medication use is generally not randomized, and thus there is a considerable risk to covary out not so much possible medication effects, but other (unknown) relevant characteristics that are associated with (particular forms of) medication use.

A different approach to understanding the effect of medication on brain activity, comes from the field of pharmacological MRI (phMRI). A promising technique to get a better insight in the effect of medication on brain activity in default state and estimate medication-induced changes of brain activity, is arterial spin labeling (ASL; Wang, Chen, Fernandez-Seara \& Detre, 2011). ASL allows for measuring the absolute blood flow and thus in contrast to BOLD provides a more quantitative estimate of blood perfusion. Taken into account the information of medication effect on baseline perfusion, it is able to determine the net effect of medication in the active state (Wang et al., 2011).

In sum, more insight must be gained in the effect of medication on brain activity found within the BPD population. Therefore it is important to report adequately the type and dose of medication, number of medications and even past use of medication. To control as much as possible for the effects of medication on brain activity one might add medication load as a regressor in the analysis or use the ASL approach.

\section{How neutral is neutral?}

To examine the neural correlates of the processing of emotional stimuli, in fMRI studies it is common to use a neutral stimuli as a control condition. The comparison of for example negative versus neutral scenes gives the opportunity to examine the emotional content of the pictures by itself and therefore seems to be the most elegant contrast. However, an essential, but somehow neglected, issue in BPD neuroimaging research concerns the interpretation of neutral stimuli in BPD patients. Experimental studies showed that BPD patients tend to interpret neutral facial expressions as more negative compared to NPC (Domes et al., 2008; Wagner \& Linehan, 1999). The authors explain this in terms of a negativity bias, suggesting that BPD patients are hypersensitive to social cues, especially 
for social exclusion and abandonment, and therefore misinterpret (social) neutral stimuli. This claim is further supported by fMRI studies showing amygdala hyperreactivity to neutral faces (Donegan et al., 2003). One should note that facial expressions are a primary source of social behavior, and BPD patients are hypersensitive to interpersonal and social cues, so therefore BPD patients would more likely interpret neutral facial expressions as more negative. Additional evidence, however, is also provided by fMRI studies using IAPS pictures (Niedtfeld et al., 2010; Schulze et al., 2011). Strikingly, ROI analyses of the amygdala by Niedtfeld et al. (2010) and Schulze et al. (2011) showed that emotional reactivity was not only related to negative social scenes, but also holds for neutral social scenes. These findings suggest that BPD patients do not perceive neutral stimuli actually as neutral. This raises the question whether it is valid to ascribe neutral stimuli as a baseline measure.

For more detailed examination instead of using a simple subtraction design it is proposed to use factorial designs allowing to analyze the interaction between variables (Friston et al., 1996). Additionally, parametric designs are often used to give different levels a separate weighting. In this case the valence of each emotional picture could be rated and used as weight in the analysis of explained variance. Results from both designs are not strongly dependent on a neutral control condition as is the case for a simple two-mean (active vs. neutral) comparison.

\section{Stimuli types}

Another avenue of research is the range of emotional stimuli used in fMRI studies in BPD. This is usually restricted to negative and neutral stimuli. However, it is also important to include other stimulus categories with a different valence such as positive stimuli to examine whether in BPD patients evidence points to a general hyperreactivity to emotional stimuli or to a specificity of negative stimuli. Two studies included positive stimuli to examine emotional sensitivity in BPD patients (Arntz et al., submitted; Koenigsberg et al., 2009b). Koenigsberg et al. (2009b) did not show altered brain activity in the limbic system (e.g. amygdala) in BPD patients compared to NPC for the contrast positive minus rest. Using the contrast positive minus neutral Arntz et al. (submitted) did also not found an increased brain response in the amygdala specific for BPD patients. In addition, they found weaker brain responses for the textual stimuli. Taken together, both studies do not support a generalized hypersensitivity to emotional stimuli in BPD patients.

Furthermore given that sexual abuse during childhood is often reported in BPD patients (e.g. Lobbestael, Arntz \& Bernstein, 2010; Zanarini et al., 2002), it is useful to examine whether stimuli with erotic content might trigger intense emotional reactions in BPD patients. Subsequently this might advocate to investigate also other BPD salient stimuli such as physical, emotional or attachment trauma, emotional experiences of abandonment or social exclusion, and anger. 


\section{Gender differences}

The majority of the presently reviewed studies included solely females (7 out of 15; Table 1) or consistent predominately of females (5 out of 15; Table 1). The main reason why so few males are included is that in mental health settings BPD is more often diagnosed in females than in males, with an estimated ratio of 3:1 respectively (American Psychiatric Association, 2013; Lieb et al., 2004). Furthermore, it is expected that the inclusion of both genders will add to the observed fMRI data variability within groups, as research shows that males and females differ in emotion processing (e.g. emotional sensitivity and regulation) (Whittle, Yucel, Yap \& Allen, 2011) and impulsivity (Li, Huang, Constable \& Sinha, 2006), and that BPD symptomology interacts with gender by inflating these differences. In line with this assumption, research indicates gender differences in the clinical presentation of BPD (Johnson et al., 2003). BPD females are more likely to display internalizing emotional behavior (e.g. feeling sad, lonely and/or anxious, social withdrawal, poor self-esteem and self-injury), compared to BPD males who engage more in externalizing, disinhibited and reactive aggressive behavior (e.g. disobeying rules, vandalism, fighting, stealing and cursing) (Johnson et al., 2003). Consequently, it seems inappropriate to investigate mixed groups without taking gender effects into account (which calls for larger samples), though only investigating females will limit the generalizability to males.

Only a few studies investigated neurobiological differences between males and females with BPD (Perez-Rodriguez et al., 2012; Soloff, Kelly, Strotmeyer, Malone \& Mann, 2003). One study showed a decreased prolactin response in BPD males compared to NPC males, which was also inversely related to impulsivity and aggression measures (Soloff et al., 2003). No differences were found between the BPD females and NPC females. These results indicated that gender differences in the serotonergic system, reflected in the prolactin response, mediate increased impulsivity in BPD males. Another study displayed gender differences in the involvement of frontal-striatal circuits in BPD patients (Perez-Rodriguez et al., 2012). Results suggested that a reduced striatal glucose metabolism in BPD males compared to BPD females and NPC is associated with difficulties in affective learning and social decision making. Together, the aforementioned studies indicate that BPD has gender-specific symptom expressions and related neurobiological responses.

In conclusion, given the predominance of female BPD patients in mental health care samples and the effect of increasing variability of already very variable fMRI datasets when both sexes are included, the focus on female BPD patients in present fMRI studies is understandable. However, at the same time differences between male and female BPD patients in emotional sensitivity, emotion regulation and impulsivity remains understudied. Consequently, future studies should directly compare male and female BPD patients, to clarify the potential role of gender in BPD. 


\section{Importance of clinical control groups}

An important issue in BPD research is the psychiatric comorbidity within BPD patients. BPD patients often show a variety of comorbid Axis I and Axis II disorders. The most common comorbidities of Axis I are major depression (MDD; 61-83\%), posttraumatic stress disorder (PTSD; 36-56\%), substance abuse (52-66\%) and eating disorders (29-53\%) (McGlashan et al., 2000; Zanarini et al., 1998a; Zimmerman \& Mattia, 1999). In terms of Axis II, 14-30\% of BPD patients have co-occurrence with paranoid personality disorder, and regarding the Cluster C personality disorders comorbidity rates are $43-47 \%$ avoidant, $16-51 \%$ dependent and 18 26\% obsessive compulsive personality disorder (McGlashan et al., 2000; Zanarini et al., 1998b). On the one hand these comorbidities are typical in BPD; 'pure' BPD patients are rare and selecting them would result in a non-representative BPD group. On the other hand without proper clinical control groups we cannot rule out effects contributed by the comorbid disorders, which calls the BPD specificity of the findings into question. Unfortunately, sample sizes for subgroup analyses controlling for specific comorbid disorders are often too small to be valid. Therefore, future fMRI studies should include additional clinical groups as for example most common comorbidities like patients with PTSD, MDD and/or Cluster C personality disorder to examine BPD specificity.

In addition, given that traumatic experiences including various abuse and neglect are very common in BPD patients (Lobbestael et al., 2010; Zanarini et al., 2002) it might be important to take trauma into account. This would also make trauma-specific and BPDspecific comparisons possible and raises understanding to what degree trauma contributes to the neural mechanisms underlying emotional processing in BPD patients. Lang et al. (2012) did not find differences in brain activity between trauma-experienced BPD patients and trauma-experienced women, and therefore suggested that trauma experiences rather than BPD modulated emotional regulation. Both groups showed different brain activity compared to NPC.

It should be noted that results could be sensitive for a combination of certain comorbidities and symptoms in subgroups of BPD patients. As mentioned earlier BPD is often associated with PTSD, childhood trauma, and dissociation, therefore comparing BPD subgroups with these comorbidities or symptoms with clinical control groups also covering these comorbidities or symptoms is essential.

Finally, an interesting clinical control group is women with premenstrual dysphoric disorder (PMDD) or premenstrual syndrome (PMS). Symptoms of PMDD/PMS are mood swings, irritability, depressed mood, feelings of hopelessness, anxiety or tension, and impairment of interpersonal functioning (American Psychiatric Association, 2013; GradyWeliky, 2003). Many of these affective symptoms are similar to emotional instability of BPD. Therefore, to examine BPD specificity PMDD/PMS is an informative control group. Of note, two fMRI studies in NPC showed that the menstrual cycle modulates brain activity of the 
amygdala, hippocampus and OFC in response to emotional stimuli (Andreano \& Cahill, 2010; Protopopescu et al., 2005). For this reason controlling for menstrual cycle might be important to take into account.

In sum, to investigate BPD specificity appropriate clinical control groups should be included in (fMRI) research designs. Moreover, it is advised to take confounding factors such as trauma and menstrual cycle into consideration.

\section{Future directions: dimensional approach}

Recently the study of basic processes that play a role in various forms of psychopathology has been promoted as a better way to gain understanding of mental disorders, than the study of categorical disorders (Cuthbert, 2014; National Institute of Mental Health, 2008). The goal of the so called Research Domain Criteria project (RDoC) is to develop new ways of classifying mental disorders based on genetics, neuroscience and behavioral science, to provide a foundation for precision diagnostics and treatment. With regard to this project, fMRI research could focus on emotional regulation problems and impulsivity in general, rather than investigate single disorders. At the moment it seems difficult to tell whether that approach will bring the field further, though there is certainly an appeal in it. One of the problems is that it is difficult to determine what the essential underlying processes are. Impulsivity, for instance, is in itself a multidimensional construct (Evenden, 1999; Moeller et al., 2001) and the impulsivity that plays a role in the type of mental health problems we currently label with BPD is not necessarily the same as in attention deficit/hyperactivity disorder (ADHD) or in antisocial PD (Moeller et al., 2001). Similarly, emotion regulation has many facets (Gross, 1998, 2007) and it is unlikely that one facet is essential for all kinds of psychopathology. Such an approach would therefore involve the development of a taxonomy of the basic processes that are relevant for different types of psychopathology. On the other hand, neuroimaging studies could play an important role in defining the essential dimensions of such constructs and help clarifying what dimension plays a role in what disorder. Consequently, this will reduce the heterogeneity, makes it is easier to define and get clear insight in the research population and might have an important contribution to the possibility of finding small effects.

\section{Conclusion}

In conclusion, emotional sensitivity, emotion regulation and impulsivity in BPD patients are important topics in neuroimaging research today. Emotional sensitivity, emotion regulation and impulsivity problems in BPD patients can be understood in terms of an impaired inhibition from the prefrontal brain areas on the limbic areas. However, the present review shows that results across the studies appear not to be that clear as previously suggested. 
By all means some conclusions need to be interpreted with caution and advocate for more studies and replications of the results. Understanding underlying neurocircuits might provide important insights in BPD and might be valuable to improve therapies and ultimately personal conditions for BPD patients and societal burden. Future studies should increase the employed sample sizes to increase statistical power and examine more subtle effects. Additionally, they should focus more on BPD specificity such as the time courses of the amygdala, different regulation strategies, manipulation of self-regulation, medication use, a wider range of stimuli (e.g. positive, non-social), gender effects and the inclusion of a clinical control group. 


\section{References}

Adolphs, R. (2001). The neurobiology of social cognition. Current Opinion in Neurobiology, 11, 231-239. doi: 10.1016/ S0959-4388(00)00202-6

Adolphs, R., \& Spezio, M. (2006). Role of the amygdala in processing visual social stimuli. Progress in Brain Research, 156, 363-378. doi: 10.1016/S0079-6123(06)56020-0

Allison, T., Puce, A., \& McCarthy, G. (2000). Social perception from visual cues: role of the STS region. Trends in Cognitive Sciences, 4, 267-278. doi: 10.1016/S1364-6613(00)01501-1

American Psychiatric Association. (2013). Diagnostic and Statistical Manual of Mental Disorder (5th ed.). Arlington, VA: American Psychiatric Association.

Anderson, I. M., Del-Ben, C. M., McKie, S., Richardson, P., Williams, S. R., Elliott, R., \& Deakin, J. F. (2007). Citalopram modulation of neuronal responses to aversive face emotions: a functional MRI study. NeuroReport, 18, 13511355. doi: 10.1097/WNR.0b013e3282742115

Andreano, J. M., \& Cahill, L. (2010). Menstrual cycle modulation of medial temporal activity evoked by negative emotion. Neurolmage, 53, 1286-1293. doi: 10.1016/j.neuroimage.2010.07.011

Arce, E., Simmons, A. N., Lovero, K. L., Stein, M. B., \& Paulus, M. P. (2008). Escitalopram effects on insula and amygdala BOLD activation during emotional processing. Psychopharmacology, 196, 661-672. doi: 10.1007/s00213-0071004-8

Arntz, A., Gielen, D., van der Heijden, P., Siep, N., Cima, M., \& van der Veen, F. (n.d.). Central and peripheral nervous system responses to visual and textual emotional stimuli in borderline personality disorder patients: specificity and effects of psychotherapy. Submitted

Baumeister, R., \& Heatherton, T. (1996). Self-regulation failure: an overview. Psychological Inquiry, 7, 1-15. doi: 10.1207/s15327965pli0701_1

Bechara, A., Damasio, H., \& Damasio, A. R. (2003). Role of the amygdala in decision-making. Annals of the New York Academy of Sciences, 985, 356-369. doi: 10.1111/j.1749-6632.2003.tb07094.x

Bland, A. R., Williams, C. A., Scharer, K., \& Manning, S. (2004). Emotion processing in borderline personality disorders. Issues in Mental Health Nursing, 25, 655-672. doi: 10.1080/01612840490486692

Breiter, H. C., Etcoff, N. L., Whalen, P. J., Kennedy, W. A., Rauch, S. L., Buckner, R. L., .. Rosen, B. R. (1996). Response and habituation of the human amygdala during visual processing of facial expression. Neuron, 17, 875-887. doi: 10.1016/S0896-6273(00)80219-6

Button, K. S., loannidis, J. P., Mokrysz, C., Nosek, B. A., Flint, J., Robinson, E. S., \& Munafo, M. R. (2013). Power failure: why small sample size undermines the reliability of neuroscience. Nature Reviews. Neuroscience, 14, 365-376. doi: $10.1038 / \mathrm{nrn} 3475$

Carpenter, R. W., \& Trull, T. J. (2013). Components of emotion dysregulation in borderline personality disorder: a review. Current Psychiatry Reports, 15, 335. doi: 10.1007/s11920-012-0335-2

Cavanna, A. E., \&Trimble, M. R. (2006). The precuneus: a review of its functional anatomy and behavioural correlates. Brain, 129, 564-583. doi: 10.1093/brain/awl004

Craig, A. D. (2009). How do you feel--now? The anterior insula and human awareness. Nature Reviews. Neuroscience, 10, 59-70. doi: 10.1038/nrn2555

Crowell, S. E., Beauchaine, T. P., \& Linehan, M. M. (2009). A biosocial developmental model of borderline personality: elaborating and extending Linehan's theory. Psychological Bulletin, 135, 495-510. doi: 10.1037/a0015616

Cuthbert, B. N. (2014). The RDoC framework: facilitating transition from ICD/DSM to dimensional approaches that integrate neuroscience and psychopathology. World Psychiatry, 13, 28-35. doi: 10.1002/wps.20087

Davidson, R. J., \& Irwin, W. (1999). The functional neuroanatomy of emotion and affective style. Trends in Cognitive Sciences, 3, 11-21. doi: 10.1016/S1364-6613(98)01265-0

Domes, G., Czieschnek, D., Weidler, F., Berger, C., Fast, K., \& Herpertz, S. C. (2008). Recognition of facial affect in borderline personality disorder. Journal of Personality Disorders, 22, 135-147. doi: 10.1521/pedi.2008.22.2.135

Domes, G., Schulze, L., \& Herpertz, S. C. (2009). Emotion recognition in borderline personality disorder-a review of the literature. Journal of Personality Disorders, 23, 6-19. doi: 10.1521/pedi.2009.23.1.6

Domes, G., Winter, B., Schnell, K., Vohs, K., Fast, K., \& Herpertz, S. C. (2006). The influence of emotions on inhibitory functioning in borderline personality disorder. Psychological Medicine, 36, 1163-1172. doi: 10.1017/ S0033291706007756 
Donegan, N. H., Sanislow, C. A., Blumberg, H. P., Fulbright, R. K., Lacadie, C., Skudlarski, P., ... Wexler, B. E. (2003). Amygdala hyperreactivity in borderline personality disorder: implications for emotional dysregulation. Biological Psychiatry, 54, 1284-1293. doi: 10.1016/S0006-3223(03)00636-X

Ebner-Priemer, U. W., Badeck, S., Beckmann, C., Wagner, A., Feige, B., Weiss, I., . . Bohus, M. (2005). Affective dysregulation and dissociative experience in female patients with borderline personality disorder: a startle response study. Journal of Psychiatric Research, 39, 85-92. doi: 10.1016/j.jpsychires.2004.05.001

Ebner-Priemer, U. W., Welch, S. S., Grossman, P., Reisch, T., Linehan, M. M., \& Bohus, M. (2007). Psychophysiological ambulatory assessment of affective dysregulation in borderline personality disorder. Psychiatry Research, 150, 265-275. doi: 10.1016/j.psychres.2006.04.014

Ekman, P., \& Friesen, W. V. (1979). Pictures of Facial Affect. Palo Alto, CA: Consulting Psychologists.

Evenden, J. L. (1999). Varieties of impulsivity. Psychopharmacology, 146, 348-361.

Fischer, H., Furmark, T., Wik, G., \& Fredrikson, M. (2000). Brain representation of habituation to repeated complex visual stimulation studied with PET. NeuroReport, 11, 123-126. doi: 10.1097/00001756-200001170-00024

Friston, K. J., Price, C. J., Fletcher, P., Moore, C., Frackowiak, R. S., \& Dolan, R. J. (1996). The trouble with cognitive subtraction. Neurolmage, 4, 97-104. doi: 10.1006/nimg.1996.0033

Frost, M. A., \&Goebel, R. (2012). Measuring structural-functional correspondence: spatial variability of specialised brain regions after macro-anatomical alignment. Neurolmage, 59, 1369-1381. doi: 10.1016/j.neuroimage.2011.08.035

Garavan, H., Ross, T. J., \& Stein, E. A. (1999). Right hemispheric dominance of inhibitory control: an event-related functional MRI study. Proceedings of the National Academy of Sciences of the United States of America, 96, 83018306. doi: 10.1073/pnas.96.14.8301

Goldin, P. R., McRae, K., Ramel, W., \& Gross, J. J. (2008). The neural bases of emotion regulation: reappraisal and suppression of negative emotion. Biological Psychiatry, 63, 577-586. doi: 10.1016/j.biopsych.2007.05.031

Goodman, M., Carpenter, D., Tang, C. Y., Goldstein, K. E., Avedon, J., Fernandez, N., .. . Hazlett, E. A. (2014). Dialectical behavior therapy alters emotion regulation and amygdala activity in patients with borderline personality disorder. Journal of Psychiatric Research, 57, 108-116. doi: 10.1016/j.jpsychires.2014.06.020

Grady-Weliky, T. A. (2003). Clinical practice. Premenstrual dysphoric disorder. The New England Journal of Medicine, 348, 433-438. doi: 10.1056/NEJMcp012067

Gross, J. J. (1998). The emerging field of emotion regulation: an integrative review. Review of General Psychology, 2, 271-299. doi: 10.1037//1089-2680.2.3.271

Gross, J. J. (2007). Handbook of Emotion Regulation. New York, NY: Guilford Publications

Guitart-Masip, M., Pascual, J. C., Carmona, S., Hoekzema, E., Berge, D., Perez, V., . . Vilarroya, O. (2009). Neural correlates of impaired emotional discrimination in borderline personality disorder: an fMRI study. Progress in Neuro-psychopharmacology \& Biological Psychiatry, 33, 1537-1545. doi: 10.1016/j.pnpbp.2009.08.022

Hafeman, D. M., Chang, K. D., Garrett, A. S., Sanders, E. M., \& Phillips, M. L. (2012). Effects of medication on neuroimaging findings in bipolar disorder: an updated review. Bipolar Disorders, 14, 375-410. doi: 10.1111/j.13995618.2012.01023.x

Hazlett, E. A., Speiser, L. J., Goodman, M., Roy, M., Carrizal, M., Wynn, J. K., . . New, A. S. (2007). Exaggerated affectmodulated startle during unpleasant stimuli in borderline personality disorder. Biological Psychiatry, 62, 250255. doi: 10.1016/j.biopsych.2006.10.028

Hazlett, E. A., Zhang, J., New, A. S., Zelmanova, Y., Goldstein, K. E., Haznedar, M. M., ... Chu, K. W. (2012). Potentiated amygdala response to repeated emotional pictures in borderline personality disorder. Biological Psychiatry, 72 , 448-456. doi: 10.1016/j.biopsych.2012.03.027

Herpertz, S. C., Dietrich, T. M., Wenning, B., Krings, T., Erberich, S. G., Willmes, K., . . Sass, H. (2001). Evidence of abnormal amygdala functioning in borderline personality disorder: a functional MRI study. Biological Psychiatry, 50, 292-298. doi: 10.1016/S0006-3223(01)01075-7

Herpertz, S. C., Kunert, H. J., Schwenger, U. B., \& Sass, H. (1999). Affective responsiveness in borderline personality disorder: a psychophysiological approach. The American Journal of Psychiatry, 156, 1550-1556.

Horn, N. R., Dolan, M., Elliott, R., Deakin, J. F., \& Woodruff, P. W. (2003). Response inhibition and impulsivity: an fMRI study. Neuropsychologia, 41, 1959-1966. doi: 10.1016/S0028-3932(03)00077-0

Jacob, G. A., Gutz, L., Bader, K., Lieb, K., Tüscher, O., \& Stahl, C. (2010). Impulsivity in borderline personality disorder: impairment in self-report measures, but not behavioral inhibition. Psychopathology, 43, 180-188. doi: $10.1159 / 000304174$ 
Jacob, G. A., Zvonik, K., Kamphausen, S., Sebastian, A., Maier, S., Philipsen, A., . . Tüscher, O. (2013). Emotional modulation of motor response inhibition in women with borderline personality disorder: an fMRI study. Journal of Psychiatry \& Neuroscience, 38, 164-172. doi: 10.1503/jpn.120029

John, O. P., \& Gross, J. J. (2004). Healthy and unhealthy emotion regulation: personality processes, individual differences, and life span development. Journal of Personality, 72, 1301-1333. doi: 10.1111/j.14676494.2004.00298.x

Johnson, D. M., Shea, M. T., Yen, S., Battle, C. L., Zlotnick, C., Sanislow, C. A., .. Zanarini, M. C. (2003). Gender differences in borderline personality disorder: findings from the Collaborative Longitudinal Personality Disorders Study. Comprehensive Psychiatry, 44, 284-292. doi: 10.1016/S0010-440X(03)00090-7

Kamphausen, S., Schroder, P., Maier, S., Bader, K., Feige, B., Kaller, C. P., . . Tüscher, O. (2013). Medial prefrontal dysfunction and prolonged amygdala response during instructed fear processing in borderline personality disorder. The World Journal of Biological Psychiatry, 14, 307-318, S301-304. doi: 10.3109/15622975.2012.665174

Kanske, P., Heissler, J., Schonfelder, S., Bongers, A., \&Wessa, M. (2011). How to regulate emotion? Neural networks for reappraisal and distraction. Cerebral Cortex, 21, 1379-1388. doi: 10.1093/cercor/bhq216

Koenigsberg, H. W., Fan, J., Ochsner, K. N., Liu, X., Guise, K. G., Pizzarello, S., ... Siever, L. J. (2009a). Neural correlates of the use of psychological distancing to regulate responses to negative social cues: a study of patients with borderline personality disorder. Biological Psychiatry, 66, 854-863. doi: 10.1016/j.biopsych.2009.06.010

Koenigsberg, H. W., Siever, L. J., Lee, H., Pizzarello, S., New, A. S., Goodman, M., . . Prohovnik, I. (2009b). Neural correlates of emotion processing in borderline personality disorder. Psychiatry Research, 172, 192-199. doi: 10.1016/j.pscychresns.2008.07.010

Krause-Utz, A., Winter, D., Niedtfeld, I., \& Schmahl, C. (2014). The latest neuroimaging findings in borderline personality disorder. Current Psychiatry Reports, 16, 438. doi: 10.1007/s11920-014-0438-z

Lang, P. J., Bradley, M. M., \& Cuthbert, B. N. (1997). International Affective Picture System (IAPS): Instruction Manual and Affective Ratings. Gainesville, FL: Center of Research in Psychophysiology, University of Florida.

Lang, S., Kotchoubey, B., Frick, C., Spitzer, C., Grabe, H. J., \& Barnow, S. (2012). Cognitive reappraisal in traumaexposed women with borderline personality disorder. Neurolmage, 59, 1727-1734. doi: 10.1016/j. neuroimage.2011.08.061

Lazarus, S. A., Cheavens, J. S., Festa, F., \& Rosenthal, M. Z. (2014). Interpersonal functioning in borderline personality disorder: a systematic review of behavioral and laboratory-based assessments. Clinical Psychology Review, 34, 193-205. doi: 10.1016/j.cpr.2014.01.007

Leichsenring, F., Leibing, E., Kruse, J., New, A. S., \& Leweke, F. (2011). Borderline personality disorder. Lancet, 377, 7484. doi: 10.1016/S0140-6736(10)61422-5

Lenzenweger, M. F., Lane, M. C., Loranger, A. W., \& Kessler, R. C. (2007). DSM-IV personality disorders in the National Comorbidity Survey Replication. Biological Psychiatry, 62, 553-564. doi: 10.1016/j.biopsych.2006.09.019

Levine, D., Marziali, E., \& Hood, J. (1997). Emotion processing in borderline personality disorders. The Journal of Nervous and Mental Disease, 185, 240-246. doi: 10.1097/00005053-199704000-00004

Leyton, M., Okazawa, H., Diksic, M., Paris, J., Rosa, P., Mzengeza, S., .. Benkelfat, C. (2001). Brain regional alpha-[11C] methyl-L-tryptophan trapping in impulsive subjects with borderline personality disorder. The American Journal of Psychiatry, 158, 775-782. doi: 10.1176/appi.ajp.158.5.775

Li, C. S., Huang, C., Constable, R. T., \& Sinha, R. (2006). Gender differences in the neural correlates of response inhibition during a stop signal task. Neurolmage, 32, 1918-1929. doi: 10.1016/j.neuroimage.2006.05.017

Lieb, K., Zanarini, M. C., Schmahl, C., Linehan, M. M., \& Bohus, M. (2004). Borderline personality disorder. Lancet, 364 , 453-461. doi: 10.1016/S0140-6736(04)16770-6

Linehan, M. M. (1993). Cognitive-Behavioral Treatment of Borderline Personality Disorder. New York: Guilford Press.

Liu, T.T., Frank, L. R., Wong, E. C., \& Buxton, R. B. (2001). Detection power, estimation efficiency, and predictability in event-related fMRI. Neurolmage, 13, 759-773. doi: 10.1006/nimg.2000.0728

Lobbestael, J., \& Arntz, A. (2010). Emotional, cognitive and physiological correlates of abuse-related stress in borderline and antisocial personality disorder. Behaviour Research and Therapy, 48, 116-124. doi: 10.1016/j. brat.2009.09.015

Lobbestael, J., Arntz, A., \& Bernstein, D. P. (2010). Disentangling the relationship between different types of childhood maltreatment and personality disorders. Journal of Personality Disorders, 24, 285-295. doi: 10.1521/ pedi.2010.24.3.285 
Lynch, T. R., Rosenthal, M. Z., Kosson, D. S., Cheavens, J. S., Lejuez, C. W., \& Blair, R. J. (2006). Heightened sensitivity to facial expressions of emotion in borderline personality disorder. Emotion, 6, 647-655. doi: 10.1037/15283542.6.4.647

Martijn, C., Alberts, H. J. E. M., \& de Vries, N. K. (2006). Maintaining self-control: the role of expectancies. In D. T. D. de Ridder \& J. B. F. de Wit (Eds.), Self-regulation in Health Behavior (pp. 169-191). Chichester, West Sussex, England: John Wiley \& Sons Ltd.

Mauchnik, J., \& Schmahl, C. (2010). The latest neuroimaging findings in borderline personality disorder. Current Psychiatry Reports, 12, 46-55. doi: 10.1007/s11920-009-0089-7

McGlashan, T. H., Grilo, C. M., Skodol, A. E., Gunderson, J. G., Shea, M. T., Morey, L. C., . . Stout, R. L. (2000). The Collaborative Longitudinal Personality Disorders Study: baseline Axis I/II and II/II diagnostic co-occurrence. Acta Psychiatrica Scandinavica, 102, 256-264. doi: 10.1034/j.1600-0447.2000.102004256.x

McRae, K., Hughes, B., Chopra, S., Gabrieli, J. D., Gross, J. J., \& Ochsner, K. N. (2010). The neural bases of distraction and reappraisal. Journal of Cognitive Neuroscience, 22, 248-262. doi: 10.1162/jocn.2009.21243

Minzenberg, M. J., Fan, J., New, A. S., Tang, C. Y., \& Siever, L. J. (2007). Fronto-limbic dysfunction in response to facial emotion in borderline personality disorder: an event-related fMRI study. Psychiatry Research, 155, 231-243. doi: 10.1016/j.pscychresns.2007.03.006

Moeller, F. G., Barratt, E. S., Dougherty, D. M., Schmitz, J. M., \& Swann, A. C. (2001). Psychiatric aspects of impulsivity. The American Journal of Psychiatry, 158, 1783-1793. doi: 10.1176/appi.ajp.158.11.1783

Morawetz, C., Holz, P., Lange, C., Baudewig, J., Weniger, G., Irle, E., \& Dechent, P. (2008). Improved functional mapping of the human amygdala using a standard functional magnetic resonance imaging sequence with simple modifications. Magnetic Resonance Imaging, 26, 45-53. doi: 10.1016/j.mri.2007.04.014

Mortensen, J. A., Rasmussen, I. A., \& Håberg, A. (2010). Trait impulsivity in female patients with borderline personality disorder and matched controls. Acta Neuropsychiatrica, 22, 139-149. doi: 10.1111/j.1601-5215.2010.00468.x

Murphy, S. E., Norbury, R., O'Sullivan, U., Cowen, P. J., \& Harmer, C. J. (2009). Effect of a single dose of citalopram on amygdala response to emotional faces. The British Journal of Psychiatry, 194, 535-540. doi: 10.1192/bjp. bp.108.056093

Nater, U. M., Bohus, M., Abbruzzese, E., Ditzen, B., Gaab, J., Kleindienst, N., ... Ehlert, U. (2010). Increased psychological and attenuated cortisol and alpha-amylase responses to acute psychosocial stress in female patients with borderline personality disorder. Psychoneuroendocrinology, 35, 1565-1572. doi: 10.1016/j.psyneuen.2010.06.002

National Institute of Mental Health. (2008). The National Institute of Mental Health Strategic Plan, from http://www. nimh.nih.gov/about/strategic-planning-reports/index.shtml

Niedtfeld, I., Schulze, L., Kirsch, P., Herpertz, S. C., Bohus, M., \& Schmahl, C. (2010). Affect regulation and pain in borderline personality disorder: a possible link to the understanding of self-injury. Biological Psychiatry, 68, 383391. doi: 10.1016/j.biopsych.2010.04.015

Ochsner, K. N., Bunge, S. A., Gross, J. J., \& Gabrieli, J. D. (2002). Rethinking feelings: an fMRI study of the cognitive regulation of emotion. Journal of Cognitive Neuroscience, 14, 1215-1229. doi: 10.1162/089892902760807212

Ochsner, K. N., Silvers, J. A., \& Buhle, J.T. (2012). Functional imaging studies of emotion regulation: a synthetic review and evolving model of the cognitive control of emotion. Annals of the New York Academy of Sciences, 1251, E124. doi: 10.1111/j.1749-6632.2012.06751.x

Olsson, A., \& Ochsner, K. N. (2008). The role of social cognition in emotion. Trends in Cognitive Sciences, 12, 65-71. doi: 10.1016/j.tics.2007.11.010

Perez-Rodriguez, M. M., Hazlett, E. A., Rich, E. L., Ripoll, L. H., Weiner, D. M., Spence, N., . . New, A. S. (2012). Striatal activity in borderline personality disorder with comorbid intermittent explosive disorder: sex differences. Journal of Psychiatric Research, 46, 797-804. doi: 10.1016/j.jpsychires.2012.02.014

Pessoa, L. (2011). Reprint of: Emotion and cognition and the amygdala: from "what is it?" to "what's to be done?". Neuropsychologia, 49, 681-694. doi: 10.1016/j.neuropsychologia.2011.02.030

Phan, K. L., Fitzgerald, D. A., Nathan, P. J., Moore, G. J., Uhde, T. W., \&Tancer, M. E. (2005). Neural substrates for voluntary suppression of negative affect: a functional magnetic resonance imaging study. Biological Psychiatry, 57, 210219. doi: 10.1016/j.biopsych.2004.10.030

Phan, K. L., Liberzon, I., Welsh, R. C., Britton, J. C., \&Taylor, S. F. (2003). Habituation of rostral anterior cingulate cortex to repeated emotionally salient pictures. Neuropsychopharmacology, 28, 1344-1350. doi: 10.1038/sj.npp.1300186

Phan, K. L., Wager, T., Taylor, S. F., \& Liberzon, I. (2002). Functional neuroanatomy of emotion: a meta-analysis of emotion activation studies in PET and fMRI. Neurolmage, 16, 331-348. doi: 10.1006/nimg.2002.1087 
Phelps, E. A. (2006). Emotion and cognition: insights from studies of the human amygdala. Annual Review of Psychology, 57, 27-53. doi: 10.1146/annurev.psych.56.091103.070234

Phillips, M. L., Drevets, W. C., Rauch, S. L., \& Lane, R. (2003). Neurobiology of emotion perception I: the neural basis of normal emotion perception. Biological Psychiatry, 54, 504-514. doi: 10.1016/S0006-3223(03)00168-9

Poldrack, R. A. (2007). Region of interest analysis for fMRI. Social Cognitive and Affective Neuroscience, 2, 67-70. doi: $10.1093 /$ scan/nsm006

Protopopescu, X., Pan, H., Altemus, M., Tuescher, O., Polanecsky, M., McEwen, B., . . Stern, E. (2005). Orbitofrontal cortex activity related to emotional processing changes across the menstrual cycle. Proceedings of the National Academy of Sciences of the United States of America, 102, 16060-16065. doi: 10.1073/pnas.0502818102

Rentrop, M., Backenstrass, M., Jaentsch, B., Kaiser, S., Roth, A., Unger, J., . . Renneberg, B. (2008). Response inhibition in borderline personality disorder: performance in a Go/Nogo task. Psychopathology, 41, 50-57. doi: 10.1159/000110626

Roepke, S., Vater, A., Preissler, S., Heekeren, H. R., \& Dziobek, I. (2012). Social cognition in borderline personality disorder. Frontiers in Neuroscience, 6, 195. doi: 10.3389/fnins.2012.00195

Rosenthal, M. Z., Gratz, K. L., Kosson, D. S., Cheavens, J. S., Lejuez, C. W., \& Lynch, T. R. (2008). Borderline personality disorder and emotional responding: a review of the research literature. Clinical Psychology Review, 28, 75-91. doi: 10.1016/j.cpr.2007.04.001

Saxe, R., Xiao, D. K., Kovacs, G., Perrett, D. I., \& Kanwisher, N. (2004). A region of right posterior superior temporal sulcus responds to observed intentional actions. Neuropsychologia, 42, 1435-1446. doi: 10.1016/j. neuropsychologia.2004.04.015

Schmahl, C. G., Elzinga, B. M., Vermetten, E., Sanislow, C., McGlashan, T. H., \& Bremner, J. D. (2003). Neural correlates of memories of abandonment in women with and without borderline personality disorder. Biological Psychiatry, 54, 142-151. doi: 10.1016/S0006-3223(02)01720-1

Schmahl, C. G., Vermetten, E., Elzinga, B. M., \& Bremner, J. D. (2004). A positron emission tomography study of memories of childhood abuse in borderline personality disorder. Biological Psychiatry, 55, 759-765. doi: 10.1016/j.biopsych.2003.11.007

Schnell, K., \& Herpertz, S. C. (2007). Effects of dialectic-behavioral-therapy on the neural correlates of affective hyperarousal in borderline personality disorder. Journal of Psychiatric Research, 41, 837-847. doi: 10.1016/j. jpsychires.2006.08.011

Schulze, L., Domes, G., Kruger, A., Berger, C., Fleischer, M., Prehn, K., ... Herpertz, S. C. (2011). Neuronal correlates of cognitive reappraisal in borderline patients with affective instability. Biological Psychiatry, 69, 564-573. doi: 10.1016/j.biopsych.2010.10.025

Sebastian, A., Jacob, G., Lieb, K., \& Tüscher, O. (2013a). Impulsivity in borderline personality disorder: a matter of disturbed impulse control or a facet of emotional dysregulation? Current Psychiatry Reports, 15, 339. doi: 10.1007/s11920-012-0339-y

Sebastian, A., Pohl, M. F., Kloppel, S., Feige, B., Lange, T., Stahl, C., ... Tüscher, O. (2013b). Disentangling common and specific neural subprocesses of response inhibition. Neurolmage, 64, 601-615. doi: 10.1016/j. neuroimage.2012.09.020

Sierra, M., \& Berrios, G. E. (1998). Depersonalization: neurobiological perspectives. Biological Psychiatry, 44, 898-908. doi: 10.1016/S0006-3223(98)00015-8

Silbersweig, D., Clarkin, J. F., Goldstein, M., Kernberg, O. F., Tuescher, O., Levy, K. N., . . Stern, E. (2007). Failure of frontolimbic inhibitory function in the context of negative emotion in borderline personality disorder. The American Journal of Psychiatry, 164, 1832-1841. doi: 10.1176/appi.ajp.2007.06010126

Singer, T., Critchley, H. D., \& Preuschoff, K. (2009). A common role of insula in feelings, empathy and uncertainty. Trends in Cognitive Sciences, 13, 334-340. doi: 10.1016/j.tics.2009.05.001

Soloff, P. H., Kelly, T. M., Strotmeyer, S. J., Malone, K. M., \& Mann, J. J. (2003). Impulsivity, gender, and response to fenfluramine challenge in borderline personality disorder. Psychiatry Research, 119, 11-24. doi: 10.1016/S0165$1781(03) 00100-8$

Trull, T. J., Jahng, S., Tomko, R. L., Wood, P. K., \& Sher, K. J. (2010). Revised NESARC personality disorder diagnoses: gender, prevalence, and comorbidity with substance dependence disorders. Journal of Personality Disorders, 24, 412-426. doi: 10.1521/pedi.2010.24.4.412

van Asselt, A. D., Dirksen, C. D., Arntz, A., \& Severens, J. L. (2007). The cost of borderline personality disorder: societal cost of illness in BPD-patients. European Psychiatry, 22, 354-361. doi: 10.1016/j.eurpsy.2007.04.001 
van Reekum, C. M., Johnstone, T., Urry, H. L., Thurow, M. E., Schaefer, H. S., Alexander, A. L., \& Davidson, R. J. (2007). Gaze fixations predict brain activation during the voluntary regulation of picture-induced negative affect. Neurolmage, 36, 1041-1055. doi: 10.1016/j.neuroimage.2007.03.052

Vogt, B. A., Vogt, L., \& Laureys, S. (2006). Cytology and functionally correlated circuits of human posterior cingulate areas. Neurolmage, 29, 452-466. doi: 10.1016/j.neuroimage.2005.07.048

Vollm, B., Richardson, P., Stirling, J., Elliott, R., Dolan, M., Chaudhry, I., .. Deakin, B. (2004). Neurobiological substrates of antisocial and borderline personality disorder: preliminary results of a functional fMRI study. Criminal Behaviour and Mental Health, 14, 39-54. doi: 10.1002/cbm.559

Wagner, A. W., \& Linehan, M. M. (1999). Facial expression recognition ability among women with borderline personality disorder: implications for emotion regulation? Journal of Personality Disorders, 13, 329-344. doi: 10.1521/pedi.1999.13.4.329

Wagner, D. D., \& Heatherton, T. F. (2013). Self-regulatory depletion increases emotional reactivity in the amygdala. Social Cognitive and Affective Neuroscience, 8, 410-417. doi: 10.1093/scan/nss082

Wang, D. J., Chen, Y., Fernandez-Seara, M. A., \& Detre, J. A. (2011). Potentials and challenges for arterial spin labeling in pharmacological magnetic resonance imaging. The Journal of Pharmacology and Experimental Therapeutics, 337, 359-366. doi: 10.1124/jpet.110.172577

Weiskopf, N., Hutton, C., Josephs, O., \& Deichmann, R. (2006). Optimal EPI parameters for reduction of susceptibilityinduced BOLD sensitivity losses: a whole-brain analysis at 3 T and 1.5 T. Neurolmage, 33, 493-504. doi: 10.1016/j. neuroimage.2006.07.029

Whalen, P. J. (1998). Fear, vigilance, and ambiguity: initial neuroimaging studies of the human amygdala. Current Directions in Psychological Science, 7, 177-188. doi: 10.1111/1467-8721.ep10836912

Whittle, S., Yucel, M., Yap, M. B., \& Allen, N. B. (2011). Sex differences in the neural correlates of emotion: evidence from neuroimaging. Biological Psychology, 87, 319-333. doi: 10.1016/j.biopsycho.2011.05.003

Wingenfeld, K., Rullkoetter, N., Mensebach, C., Beblo, T., Mertens, M., Kreisel, S., . . Woermann, F. G. (2009). Neural correlates of the individual emotional stroop in borderline personality disorder. Psychoneuroendocrinology, 34, 571-586. doi: 10.1016/j.psyneuen.2008.10.024

Wright, C. I., Fischer, H., Whalen, P. J., Mclnerney, S. C., Shin, L. M., \& Rauch, S. L. (2001). Differential prefrontal cortex and amygdala habituation to repeatedly presented emotional stimuli. NeuroReport, 12, 379-383. doi: 10.1097/00001756-200102120-00039

Zanarini, M. C., Frankenburg, F. R., Dubo, E. D., Sickel, A. E., Trikha, A., Levin, A., \& Reynolds, V. (1998a). Axis I comorbidity of borderline personality disorder. The American Journal of Psychiatry, 155, 1733-1739.

Zanarini, M. C., Frankenburg, F. R., Dubo, E. D., Sickel, A. E., Trikha, A., Levin, A., \& Reynolds, V. (1998b). Axis II comorbidity of borderline personality disorder. Comprehensive Psychiatry, 39, 296-302. doi: 10.1016/S0010-440X(98)90038-4

Zanarini, M. C., Frankenburg, F. R., Hennen, J., \& Silk, K. R. (2004). Mental health service utilization by borderline personality disorder patients and Axis II comparison subjects followed prospectively for 6 years. The Journal of Clinical Psychiatry, 65, 28-36.

Zanarini, M. C., Ruser, T., Frankenburg, F. R., \& Hennen, J. (2000). The dissociative experiences of borderline patients. Comprehensive Psychiatry, 41, 223-227. doi: 10.1016/S0010-440X(00)90051-8

Zanarini, M. C., Yong, L., Frankenburg, F. R., Hennen, J., Reich, D. B., Marino, M. F., \& Vujanovic, A. A. (2002). Severity of reported childhood sexual abuse and its relationship to severity of borderline psychopathology and psychosocial impairment among borderline inpatients. The Journal of Nervous and Mental Disease, 190, 381387. doi: 10.1097/00005053-200206000-00006

Zimmerman, M., \& Mattia, J. I. (1999). Axis I diagnostic comorbidity and borderline personality disorder. Comprehensive Psychiatry, 40, 245-252. doi: 10.1016/S0010-440X(99)90123-2 



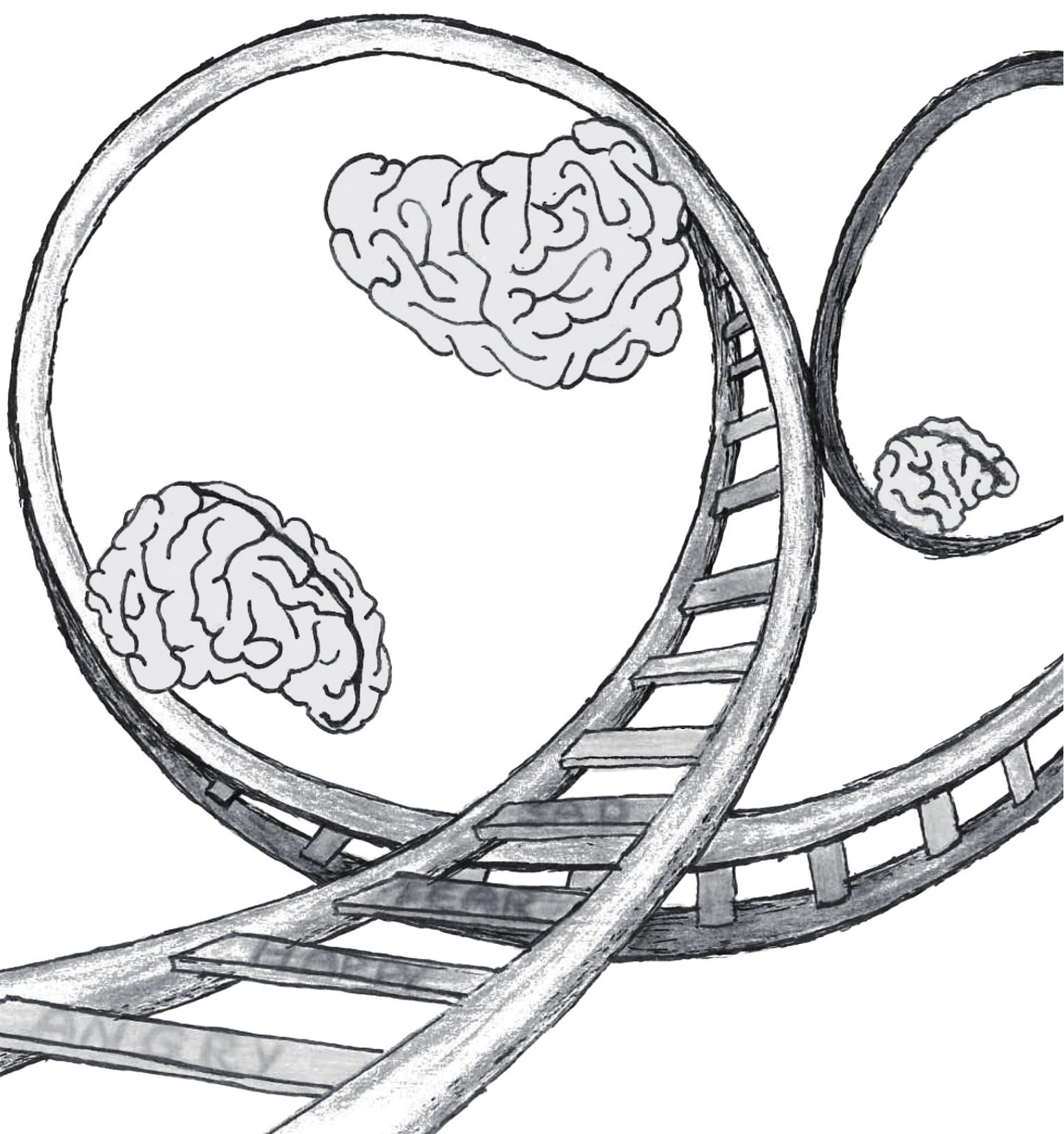




\section{Always on guard: Emotion regulation in borderline personality disorder compared to non-patients and cluster-c personality disorder patients}

This chapter has been submitted as:

Van Zutphen, L., Siep, N., Jacob, G.A., Domes, G., Sprenger, A., Willenborg, B., Goebel, R. \& Arntz, A. (n.d.). Always on guard: Emotion regulation in borderline personality disorder compared to non-patients and cluster-c personality disorder patients. Submitted 


\section{Abstract \\ Background}

We investigated neural correlates of hypothesized increased emotional sensitivity and impaired emotion regulation of borderline personality disorder (BPD) patients to negative, positive and erotic stimuli, by comparing them to non-patients and cluster-C PD patients.

\section{Methods}

During fMRI scanning, 55 BPD patients, 42 non-patients and 24 cluster-C PD patients completed an emotion regulation task, including social pictures. As regulation strategy we instructed participants to realize they were safe, inspired by schema therapy theory.

\section{Results}

Passive viewing of negative stimuli resulted in higher activity in the anterior insula, temporoparietal junction and dorsolateral prefrontal cortex in BPD patients compared to non-patients. The increased activity in the anterior insula and temporoparietal junction was not specific for negative stimuli, but was also present when BPD patients viewed positive stimuli. During regulation of negative stimuli, non-patients showed higher activity in the dorsal anterior cingulate cortex, dorsolateral prefrontal cortex, middle temporal gyrus and bilateral inferior parietal lobule. BPD patients did not show this increase in activity. Overall, cluster-C patients showed activity that was in-between BPD and non-patients.

\section{Conclusions}

When looking at emotional stimuli BPD patients showed a unique pattern of activity, suggesting an increase in brain activity involved in mentalizing processes and emotional bodily states. In the case of negative stimuli this is accompanied by increased activity in regulation areas. In contrast, increase of regulation processes seems absent when BPD patients are explicitly instructed to regulate. Results of diagnosis specificity support a dimensional rather than a dichotomous differentiation between BPD and control patients regarding emotional sensitivity and emotion regulation of social stimuli.

Keywords: BPD, Emotional sensitivity, Emotion regulation, Emotional instability, Emotion reactivity, $\mathrm{fMRI}$ 


\section{Introduction}

The life of a borderline personality disorder (BPD) patient can be described as an emotional rollercoaster, as it is characterized by a pervasive pattern of instability in affect regulation and impulse control (American Psychiatric Association, 2013). BPD is a life threatening illness, affecting 1-3\% of the general population (Lenzenweger, Lane, Loranger \& Kessler, 2007; Trull, Jahng, Tomko, Wood \& Sher, 2010) and, is associated with high rates of selfinjury, suicidal tendencies and reactive aggression (Leichsenring, Leibing, Kruse, New \& Leweke, 2011). Apart from the severe functional impairments, extensive use of healthcare treatments results in high societal costs (Leichsenring et al., 2011; van Asselt, Dirksen, Arntz \& Severens, 2007).

Leading theories of BPD (Koenigsberg et al., 2009b; Linehan, 1993) propose that emotional instability can be best explained in terms of an increase in emotional sensitivity and impairments to regulate emotional responses. Supporting these theories, empirical neurobiological studies show that BPD patients have increased activity in the limbic brain areas, involved in emotion generation, and decreased activity in the prefrontal cortex, involved in regulatory processes (Krause-Utz, Winter, Niedtfeld \& Schmahl, 2014; Mauchnik \& Schmahl, 2010; Schulze, Schmahl \& Niedtfeld, 2015). Unfortunately, research findings here-to-date are rather inconclusive and inconsistent (van Zutphen, Siep, Jacob, Goebel \& Arntz, 2015), and important issues remain unanswered. First, it remains unclear whether the increased emotional sensitivity is restricted to negative emotions, as nearly all previous studies used negative emotional stimuli only, or whether it also involves other types of emotions. Second, it remains uncertain whether reported findings are specific for BPD or rather characteristic of psychopathology in general, as previous studies often lack clinical control groups. Third, most studies were statistically underpowered (van Zutphen et al., 2015), possibly an important cause of inconsistency in reported findings. In order to improve our understanding of BPD, future research should address these issues.

In the present international, multicenter fMRI study we investigated emotional sensitivity and emotion regulation abilities in BPD with a focus on stimulus category specificity and diagnosis specificity. We used an emotion sensitivity and regulation paradigm (Koenigsberg et al., 2009a; Ochsner, Bunge, Gross \& Gabrieli, 2002; Schulze et al., 2011) with different categories of emotional social stimuli. As regulation strategy we instructed participants to realize they were safe, inspired by schema therapy theory (Arntz \& van Genderen, 2009). We extended previous research by adding positive stimuli, and given the high rates of childhood sexual abuse (Zanarini et al., 2002) and relationship problems (American Psychiatric Association, 2013) in BPD erotic stimuli were added. We expected erotic stimuli to evoke emotional responses similar to the negative stimuli in BPD. As control groups we used nonpatients (NPC) and Cluster-C personality disorder patients (CCP). Following the model of cognitive control of emotion (Ochsner, Silvers \& Buhle, 2012), we hypothesized that BPD 
patients would show higher activity in emotion generation brain areas (amygdala, ventral striatum, anterior insula, ventromedial prefrontal cortex) when passively viewing negative and erotic pictures, compared to both control groups. Additionally, we expected that BPD patients would show decreased activity in emotion regulation brain areas (dorsolateral (dIPFC), posterior, ventrolateral (vIPFC), and dorsomedial prefrontal cortex (dmPFC), dorsal anterior cingulate cortex (dACC), inferior parietal cortex), whilst regulating emotions during the presentation of especially negative and erotic pictures, compared to NPC and CCP.

\section{Materials and Methods}

\section{Participants}

Sixty-two BPD patients, 48 NPC and 31 CCP were scanned. Four BPD patients, one NPC and one CCP were excluded because of invalid or incomplete data, one BPD and one CCP because of too much head motion (if head motion in 2 or more runs was $>4 \mathrm{~mm}$ ) during scanning, three NPC because of scores above 70 on the Brief Symptom Inventory and three CCP because of scores above 100 on the BPD checklist. Two BPD and two CCP were excluded because they had an estimated IQ outside the range of 70-120. Additionally, as there was a disproportionate number of NPC with IQ higher than the highest IQ in the BPD patients, we excluded two NPC with IQ scores above the 95-percentile to guarantee that NPC were matched to the BPD patients. After exclusion of participants not meeting scanning or clinical criteria, 55 BPD patients, 42 NPC and 24 CCP were left for the analyses, implying that this study was powered at $80 \%$ to detect a large effect size $(d>.76)$ between groups at $p=.005$.

Participants were recruited from two sites in the Netherlands (Maastricht, Heerlen) and three sites in Germany (Freiburg, Lübeck, Hamburg). Patients were recruited from mental health clinics at local sites, Virenze-Riagg Maastricht (The Netherlands), PsyQ Heerlen (The Netherlands), the BPD treatment unit of the Department of Psychiatry and Psychotherapy at the Medical Center Freiburg (Germany), the Department of Psychiatry and Psychotherapy at the University Hospital Lübeck (Germany) and the Institute for Behavior Therapy Training Hamburg (Germany). BPD patients were recruited within the context of an international multicenter RCT on group schema therapy versus treatment-as-usual (Wetzelaer et al., 2014). NPC were recruited among the general population at each site via postings and personal contacts. Participants had to be hetero- or bisexual females, aged 18-65, sufficient understanding of the language at the local sites. Only females were chosen since gender might influence emotional processing (Whittle, Yucel, Yap \& Allen, 2011), and because in mental health care BPD is more often diagnosed in females. We excluded homosexual females, because we used heterosexual erotic stimuli. General exclusion criteria were lifetime psychotic or bipolar disorder type-I, attention-deficit/hyperactivity disorder, dissociative 
identity disorder, serious and/or unstable medical illness, substance dependence needing clinical detoxification and fMRI exclusion criteria (i.e. claustrophobia, metal objects, cardiac arrhythmia, epilepsy, tattoos at neck/head and pregnancy).

BPD and CCP patients were diagnosed according to the DSM-IV criteria, using the Structural Clinical Interview (SCID) II (First, Spitzer, Gibbon, Williams \& Benjamin, 1997) and I (First, Spitzer, Gibbon \& Williams, 1994), assessed by trained interviewers. Preferably patients did not start treatment yet. If the patient started treatment, measurements had to be finished within three months from the start of therapy. BPD patients were further screened by means of the BPD Severity Index (Arntz et al., 2003; Giesen-Bloo, Wachters, Schouten \& Arntz, 2010; Kroger et al., 2013), for inclusion this score was $>20$ (31.83 \pm 7.32$)$. Narcissistic and antisocial personality disorders, full or sub-threshold, were excluded for reasons related to the clinical trial in which this study sample participated (Wetzelaer et al., 2014). CCP were not allowed to score full or sub-threshold Cluster-B personality disorder and > 2 BPD criteria. NPC did not meet current diagnostic criteria for Axis I or Axis II disorders, assessed with SCID-I and II screeners (First et al., 1994; First et al., 1997). Positive items on screeners were checked with SCID interviews. Additional assessments included the Brief Symptom Inventory (Derogatis, 1993), BPD checklist (Arntz \& Dreessen, 1995) and Interview for Trauma Events in Childhood (Lobbestael, Arntz, Harkema-Schouten \& Bernstein, 2009). See supplemental materials for details measurement descriptions. Table 1 presents demographic and clinical data of all groups. The groups did not significantly differ concerning age, handedness and IQ.

After complete description of the study, written informed consent was obtained. Participants received a small financial remuneration. The study was approved by the local medical ethical committees (Wetzelaer et al., 2014).

\section{Experimental task}

We used an adapted version of a classic emotion regulation paradigm with pictorial stimuli (Koenigsberg et al., 2009a; Ochsner et al., 2002; Schulze et al., 2011). Present paradigm (Figure 1) included a traditional Look condition, requiring participants to attend to the pictures and respond naturally without altering their emotional state. Our adapted regulation condition was the so called Safe condition, in which participants were instructed to realize themselves being safe. This regulation strategy was inspired by schema therapy, a highly effective therapy for BPD (Sempertegui, Karreman, Arntz \& Bekker, 2013). Based on one of the components of schema therapy that 'unmet safety needs during childhood' underlie emotional problems, we hypothesized that BPD patients feel unsafe while experiencing negative emotions (Arntz \& van Genderen, 2009), even when realizing they are in a currently safe situation. 
Table 1. Descriptive statistics for demographic and clinical variables across the three groups.

\begin{tabular}{|c|c|c|c|c|c|}
\hline & \multirow{2}{*}{$\begin{array}{l}\text { BPD } \\
(n=55)\end{array}$} & \multirow{2}{*}{$\begin{array}{l}\text { NPC } \\
(n=42)\end{array}$} & \multirow{2}{*}{$\begin{array}{l}\text { CCP } \\
(n=24)\end{array}$} & \multicolumn{2}{|c|}{ Test Statistics } \\
\hline & & & & $F$ & $p$ \\
\hline Age, years, mean (SD) & $30.80(8.78)$ & $28.33(10.50)$ & $30.38(11.46)$ & .77 & .465 \\
\hline Education levela, No. (\%) & & & & $8.90^{\mathrm{b}}$ & $.012^{\mathrm{b}}$ \\
\hline Level 1 & $13(23.6)$ & $7(16.7)$ & $4(16.7)$ & & \\
\hline Level 2 & $8(14.5)$ & $2(4.8)$ & $4(16.7)$ & & \\
\hline Level 3 & $15(27.3)$ & $4(9.5)$ & $7(29.2)$ & & \\
\hline Level 4 & $3(5.5)$ & $2(4.8)$ & $3(12.5)$ & & \\
\hline Level 5 & $13(23.6)$ & $19(45.2)$ & $3(12.5)$ & & \\
\hline Level 6 & $3(5.5)$ & $7(16.7)$ & $3(12.5)$ & & \\
\hline Estimated IQc mean (SD) & $96.66(9.93)$ & $100.43(11.03)$ & $98.05(9.42)$ & 1.59 & .208 \\
\hline Handedness, No. L/R/M & $5 / 46 / 3$ & $2 / 40 /-$ & $-/ 24 /-$ & $6.72^{d}$ & $.151^{\circ}$ \\
\hline $\mathrm{BSI}$, mean (SD), total & $1.74(.57)$ & $.13(.13)$ & $1.08(.45)$ & 150.74 & $<.001^{\mathrm{e}}$ \\
\hline BPD checklist, mean (SD), total & $120.60(26.92)$ & $50.73(5.03)$ & $72.96(17.02)$ & 147.41 & $<.001^{\mathrm{f}}$ \\
\hline ITEC, mean (SD) & & & & 9.90 & $<.0019$ \\
\hline Sexual abuse & $9.10(8.95)$ & $.13(.41)$ & $1.97(5.01)$ & 21.57 & $<.001$ \\
\hline Physical abuse & $16.93(11.77)$ & $1.63(3.51)$ & $7.12(10.55)$ & 27.41 & $<.001$ \\
\hline Emotional abuse & $20.36(8.53)$ & $2.32(3.27)$ & $12.92(8.50)$ & 63.46 & $<.001$ \\
\hline Emotional neglect & $10.89(6.95)$ & $.77(2.02)$ & $6.37(6.46)$ & 32.19 & $<.001$ \\
\hline Physical neglect & $9.99(9.08)$ & $.82(2.74)$ & $4.76(6.99)$ & 17.17 & $<.001$ \\
\hline Dissociation, mean (SD) & & & & 8.97 & $<.001^{\mathrm{h}}$ \\
\hline prior scanning & $20.16(19.44)$ & $5.29(6.51)$ & $7.04(8.34)$ & 14.46 & $<.001$ \\
\hline post scanning & $31.86(26.11)$ & $6.69(8.36)$ & $16.60(20.65)$ & 17.45 & $<.001$ \\
\hline Anxiety, mean (SD) & & & & 7.68 & $<.001^{\mathrm{i}}$ \\
\hline prior scanning & $41.90(30.59)$ & $10.00(20.03)$ & $30.87(30.91)$ & 15.37 & $<.001$ \\
\hline post scanning & $25.60(27.17)$ & $5.93(14.75)$ & $23.70(32.86)$ & 7.66 & .001 \\
\hline Nervousness, mean (SD) & & & & 10.85 & $<.001^{\mathrm{j}}$ \\
\hline prior scanning & $54.75(32.28)$ & $15.68(21.94)$ & $36.78(30.67)$ & 20.90 & $<.001$ \\
\hline post scanning & $27.49(27.14)$ & $6.05(13.90)$ & $30.17(36.52)$ & 9.79 & $<.001$ \\
\hline Axis I disorders, No. (\%) & & & & & $p^{k}$ \\
\hline Major depressive disorder & $49(89.1)$ & & $13(54.2)$ & & .001 \\
\hline Dysthymic & $4(7.3)$ & & $1(4.2)$ & & .602 \\
\hline Bipolar type II & $1(1.8)$ & & - & & .506 \\
\hline Generalized anxiety disorder & $2(3.6)$ & & $1(4.2)$ & & .910 \\
\hline Panic disorder with agoraphobia & $7(12.7)$ & & $2(8.3)$ & & .572 \\
\hline Panic disorder & $7(12.7)$ & & $3(12.5)$ & & .978 \\
\hline Agoraphobia & $4(7.3)$ & & - & & .175 \\
\hline Specific phobia & $10(18.2)$ & & $1(4.2)$ & & .098 \\
\hline Social phobia & $19(34.5)$ & & $6(25.0)$ & & .402 \\
\hline Obsessive compulsive disorder & $8(14.5)$ & & $2(8.3)$ & & .445 \\
\hline Posttraumatic stress disorder & $20(36.4)$ & & $3(12.5)$ & & .032 \\
\hline Somatoform disorder & $5(9.1)$ & & $4(16.7)$ & & .330 \\
\hline Eating disorders & $22(40.0)$ & & $8(33.3)$ & & .574 \\
\hline
\end{tabular}


Table 1. Descriptive statistics for demographic and clinical variables across the three groups. (Continued)

\begin{tabular}{|c|c|c|c|c|c|}
\hline & \multirow{2}{*}{$\begin{array}{l}\text { BPD } \\
(n=55)\end{array}$} & \multirow{2}{*}{$\begin{array}{l}\text { NPC } \\
(n=42)\end{array}$} & \multirow{2}{*}{$\begin{array}{l}\text { CCP } \\
(n=24)\end{array}$} & \multicolumn{2}{|c|}{ Test Statistics } \\
\hline & & & & $F$ & $p$ \\
\hline Substance abuse & $27(49.1)$ & & $1(4.2)$ & & $<.001$ \\
\hline Intermitted explosive disorder & $1(1.8)$ & & - & & .506 \\
\hline \multicolumn{6}{|l|}{ Axis II disorders, No. (\%) } \\
\hline Avoidant PD & $27(49.1)$ & & $17(70.8)$ & & .074 \\
\hline Dependent PD & $10(18.2)$ & & $2(8.3)$ & & .262 \\
\hline Obsessive compulsive PD & $11(20.0)$ & & $8(33.3)$ & & .202 \\
\hline Passive aggressive PD & $4(7.3)$ & & - & & .175 \\
\hline Depressive PD & $15(27.3)$ & & $2(8.3)$ & & .060 \\
\hline Paranoid PD & $15(27.3)$ & & - & & .004 \\
\hline Schizotypal PD & $1(1.8)$ & & - & & .506 \\
\hline Schizoid PD & $1(1.8)$ & & - & & .506 \\
\hline \multicolumn{6}{|l|}{ Medication, No. (\%) } \\
\hline Antidepressants & $37(67.3)$ & & $9(37.5)$ & & .014 \\
\hline Antipsychotics & $8(14.3)$ & & - & & .049 \\
\hline Hypnotics & $3(5.5)$ & & - & & .243 \\
\hline Mood Stabilizers & $1(1.8)$ & & - & & .506 \\
\hline
\end{tabular}

Abbreviations: $\mathrm{L}=$ = Left; $\mathrm{R}=$ Right; $\mathrm{M}=$ Mixed; BSI = Brief Symptom Inventory; BPD checklist = Borderline checklist; ITEC = Interview Traumatic Events Childhood; PD = Personality Disorders.

a Level of education of both the Dutch and German educational systems were translated into the International Standard Classification of Education (ISCED), in current study six levels of education were divided ranging from lower secondary school to Master's degree.

${ }^{b}$ Value is based on the Kruskal-Wallis test, data of one NPC not available.

c Assessed with four subtasks of the WAIS, data of one NPC not available

${ }^{d}$ Value is based on Chi-square, data of one BPD patient not available.

e All three groups significantly differed from each other $(p<.001)$, data of two NPC not available.

${ }^{f}$ All three groups significantly differed from each other $(p<.001)$, data of two NPC and one CCP not available.

${ }^{9}$ MANOVA and ANOVAs showed significant group effects over traumas. BPD patients experienced significantly more trauma compared to both control groups regarding sexual abuse (both groups $p<.001$ ), physical abuse (both groups $p$ $<.001$ ) and physical neglect (vs. NPC $p<.001$; vs. CCP $p=.014$ ). The three groups significantly differed from each other concerning emotional abuse $(p<.001)$ and emotional neglect (BPD vs. NPC $p<.001$; BPD vs. CCP $p=.006$; NPC vs. CCP $p$ $=.002$ ), with BPD patients experiencing the most trauma, followed by the CCP and the NPC experienced the least trauma. Data of eight NPC and one CCP not available.

${ }^{\text {h }}$ Available data of 51 BPD patients, 40 NPC and 23 CCP. MANOVA and ANOVAs showed significant group effects over dissociation. BPD patients dissociated significantly more prior and post scanning compared to both control groups (prior scanning: BPD vs. NPC $p<.001$, BPD vs. CCP $p=.001$; post scanning: BPD vs. NPC $p<.001$, BPD vs. NPC $p=.011$ ). Repeated measures ANOVA showed a significant group $x$ dissociation interaction $\left[F_{2.11}=5.27 ; p=.007\right]$. BPD patients dissociated significantly more post scanning versus prior compared to NPC $(p=.006)$.

' Available data of 52 BPD patients, 40 NPC and 23 CCP. MANOVA and ANOVAs showed significant group effects over anxiety. NPC were significantly less anxious prior and post scanning compared to both other groups (prior scanning: BPD vs. NPC $p<.001$, NPC vs. CCP $p=.013$; post scanning: BPD vs. NPC $p=.001$, NPC vs. CCP $p=.023$ ). Repeated measures ANOVA showed no significant group $x$ anxiety interaction $\left[F_{2.112}=2.12 ; p=.125\right]$.

javailable data of 51 BPD patients, 40 NPC and 23 CCP. MANOVA and ANOVAs showed significant group effects over nervousness. All three groups significantly differed from each other in their nervousness prior scanning, BPD patients were most nervous, followed by the CCP and next the NPC (BPD vs. NPC $p<.001$, BPD vs. CCP $p=.042$ and NPC vs CCP $p=.017$ ). NPC were significantly less nervous post scanning compared to both other groups (BPD vs. NPC $p<.001$. NPC Vs. CCP $p=.002)$. Repeated measures ANOVA showed a significant group $x$ nervousness interaction $\left[F_{2,111}=5.57 ; p=.005\right]$. BPD patients were significantly more nervous post scanning versus prior compared to $\operatorname{NPC}(p=.016)$.

${ }^{k}$ Value is based on Chi-square. 
In other words, whilst NPC would be able to use the Safe instruction to regulate negative emotions, BPD patients would have problems in using this to regulate emotions as negative emotions are intrinsically threatening for them. (A forthcoming study investigates whether the ability of BPD patients to profit from realizing the present situation is safe improves along treatment.) The two conditions were presented in a pseudo-randomized order (no more than three identical conditions in a row) which, was used for all participants. Preceded by an instruction cue, participants were presented with a picture. Just after the picture disappeared, participants assessed their momentary emotional state using a horizontal -100 to $100 \mathrm{~mm}$ visual analogue scale. The task consisted of 96 trials divided into four runs of 24 trials each. After scanning, participants evaluated arousal and valence of each presented picture, using the Self-Assessment Manikin Scale (Bradley \& Lang, 1994).

We used four stimulus categories containing: 24 negative, 24 neutral, 24 positive and 24 erotic pictures. Pictures were selected from the International Affective Picture System (Lang, Bradley \& Cuthbert, 1997), and additional erotic pictures from Jacob et al. (2011). Only pictures with social content were selected, since BPD patients are particularly responsive to interpersonal stimuli (Koenigsberg et al., 2009a). Pictures were randomly presented per participant and balanced across condition types. Presentation of the stimuli and recordings of behavioral responses were controlled by Presentation (Neurobehavioral Systems Inc., Albany, CA, USA). The visual stimuli were projected via PC and beamer onto a screen that was viewed through a mirror on the headcoil or via a goggle-system.

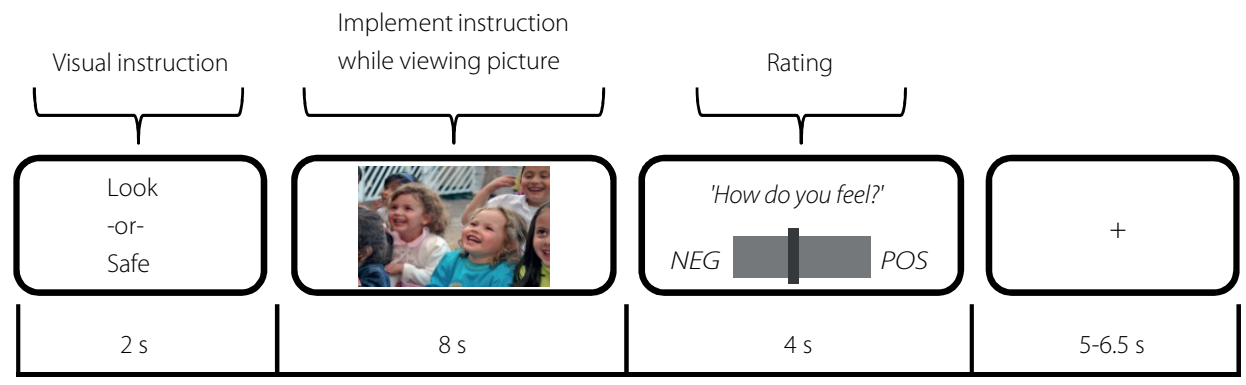

Figure 1. Schematic overview of a single trial of the task

Each 19-20.5 s trial consisted of a $2 \mathrm{~s}$ visual instruction to either 'look' or 'realize being safe', a $8 \mathrm{~s}$ presentation of the picture for carrying out the instruction, a $4 \mathrm{~s}$ rating period and a 5-6.5 s fixation (relax). During the rating period, participants indicated their emotional experience at the moment moving the pointer on the horizontal scale using a button box between negative (-100) to positive (100). 


\section{Procedure}

To ensure correct and confident use of the emotion regulation strategy and the nature of the stimuli, prior to scanning the participant was trained with a practice task outside the scanner. This task was similar to the experimental task inside the scanner. After completing the practice task the participant entered the scanner and the scanning session was performed, lasting 75 minutes. As part of the scanning session participants also underwent two resting state scans (results reported separately Baczkowski et al., 2016). Before and after scanning the anxiety and dissociation state was assessed (Stiglmayr, Shapiro, Stieglitz, Limberger \& Bohus, 2001). In addition, at the end of the session the participant completed an 'exit'-questionnaire, assessing information about a description of which strategy the participant used concerning both instructions (look and safe) and general experience with the fMRI-measurement. Finally, the participants had to rate their subjective reaction of each picture they had seen during the session.

\section{fMRI acquisition}

Functional MRI was performed on 3-Tesla scanners at the three sites, at Maastricht on a Siemens Magnetom Allegra head-only scanner equipped with a birdcage headcoil (Siemens Medical Systems, Erlangen, Germany), at Freiburg on a Siemens tim-Trio Magnetom whole body scanner (Siemens Medical Systems, Erlangen, Germany) equipped with an 8-channel headcoil, and at Lübeck on a Philips Achiva whole body scanner equipped with an 8-channel headcoil (Philips Healthcare, Best, The Netherlands). The BPD patients from Heerlen were scanned in Maastricht and the BPD patients from Hamburg were scanned in Lübeck. In Maastricht 13 BPD, 10 NPC and 11 CCP were scanned, Freiburg scanned 14 BPD, 18 NPC and 8 CCP, finally 28 BPD, 14 NPC and 5 CCP were scanned in Lubeck.

Participants were scanned in head first supine position. Head movements were minimalized using foam paddings. Additionally, participants were instructed not to look away from the stimuli or to close her eyes, and avoid moving as much as possible during scanning. T2*-weighted images were acquired via echo planar imaging (EPI), using the following imaging parameters: repetition time $=2000 \mathrm{~ms}$, echo time $=27 \mathrm{~ms}$, flip angle $=90^{\circ}$, field of view $=192 \times 192 \mathrm{~mm}$, voxel size $=3 \times 3 \times 3 \mathrm{~mm}$, and matrix $=64 \times 64$. Images were recorder in four runs of 240 images in Maastricht and 252 images in Freiburg and Lübeck. One volume in Maastricht consisted of 32, and in Freiburg and Lübeck of 34, interleaved measured axial slices. The $\mathrm{T} 2^{*}$-weighted slices were optimized with a negative tilt of $30^{\circ}$, to minimize susceptibility and distortion artifacts within the amygdala (Morawetz et al., 2008) in Maastricht and Freiburg. A whole brain anatomical scan in sagittal plane was acquired, using a high resolution T1-weigthed sequence (repetition time $=2250 \mathrm{~ms}$, echo time $=2.6 \mathrm{~ms}$, flip angle $=9^{\circ}$, field of view $=256 \times 256 \mathrm{~mm}$, voxel size $1 \times 1 \times 1 \mathrm{~mm}$ ). In total, 192 images were obtained in Maastricht, 160 in Freiburg and 170 in Lübeck. 


\section{fMRI preprocessing}

All preprocessing and statistical analyses were performed with BrainVoyager 2.6 (Brain Innovation, Maastricht, The Netherlands). The first two images of each run were discarded because of saturation effects. Preprocessing comprised slice time correction with sinc interpolation, 3D motion correction for three translation and three rotation parameters with trilinear interpolation for detection, and sinc interpolation for motion correction and removal of low-frequency drifts was performed by high-pass temporal filtering of 2 sines/ cosines per run (Goebel, Esposito \& Formisano, 2006). In all anatomical scans, the brain was peeled from the skull and corrected for intensity inhomogeneities. Participants underwent a second session concerning another task (data presented elsewhere van Zutphen et al., submitted), in which also an anatomical scan was conducted. To obtain a high resolution and high contrast anatomical scan, both anatomical scans were averaged when possible. After preprocessing the functional data were coregistered with the anatomical data per run, and for each run a volume-time-course was created. Each volume-time-course was spatially smoothed with a $6 \mathrm{~mm}$ full-width-at-half-maximum isotropic Gaussian kernel. Spatial normalization was performed using standard Talairach transformation procedures (Talairach and Tournoux, 1988).

\section{Data analyses}

To model the hemodynamic response the applied general linear model included 10 predictors: instruction, negative-look, positive-look, erotic-look, neutral-look, negative-safe, positive-safe, erotic-safe, neutral-safe and ratings. Additionally, six motion parameters $(x, y$, z-translation and $x, y, z$-rotation) were added as confound predictors.

As specificity of differences in brain activity between BPD and NPC during emotional sensitivity and emotion regulation concerning negative stimuli were the primary focus of this study, for each participant statistical parametric maps were calculated from contrasts of interest: 1) negative-look versus neutral-look, and 2) negative-safe versus negative-look. These contrast images were entered into group-level analyses, including two betweensubject factors; group (BPD, NPC) and site (Maastricht, Freiburg, Lubeck). Subsequently, two whole-brain random-effects (RFX) ANOVA were carried out: 1) stimuli (negative-look vs. neutral-look) $x$ group (BPD vs. NPC) to examine differences in emotional sensitivity, and 2) instruction (negative-safe vs. negative-look) x group (BPD vs. NPC) to investigate differences in emotion regulation. The resulting F-maps were thresholded at a significance level of $p<$ .005 and cluster-size, which seemed an adequate compromise of reducing type-I and II errors (Lieberman \& Cunningham, 2009). The minimal cluster-size threshold, being 15 voxels for the emotional sensitivity F-map and 20 voxels for the emotion regulation F-map, was determined with the cluster-level statistical threshold estimation tool in BrainVoyager that implements a Monte Carlo simulation-based approach of cluster-level correction of multiple 
comparisons (1000 simulations: Forman et al., 1995). Detailed analyses of the resulting clusters was performed in SPSS 21 (IBM Corporation, New York), using the extracted mean cluster beta of each predictor per participant.

To investigate BPD-specificity of the results, mean beta values per cluster of the CCP were additionally extracted from the active clusters and used in post-hoc comparisons. The same strategy was applied to examine BPD response uniqueness to negative stimuli, for mean beta's per cluster of positive and erotic stimuli. Finally, each cluster was checked for confounding effects of medication, within BPD [stimulus or instruction $\mathrm{x}$ medication (medicated vs. non-medicated)].

\section{Results}

\section{Manipulation checks}

Supporting the assumption that task instructions were implemented successfully, subjects' ratings demonstrated a significant interaction of task instruction (look vs. safe) $x$ stimulus category (negative, positive, erotic vs. neutral) $\left[F_{3,116}=4.41 ; p=.006\right]$. Neutral and negative, but not positive and erotic stimuli were experienced more pleasant during the safe versus look condition across groups.

Stimulus evaluations after scanning confirmed that negative stimuli were most arousing across groups, followed by erotic stimuli, positive and neutral stimuli were equally least arousing $\left[F_{3,85}=67.38 ; p<.001\right]$. Valence ratings confirmed that negative stimuli were evaluated as most unpleasant, followed by neutral stimuli, erotic stimuli and last positive stimuli $\left[F_{3.85}=297.39 ; p<.001\right]$. See supplemental materials for details of self-reported emotional state during scanning and stimulus evaluations after scanning.

\section{fMRI results of emotional sensitivity}

The whole-brain RFX ANOVA F-map: stimulus (negative-look vs. neutral-look) x group (BPD vs. NPC) resulted in four significant clusters, which were identified the right anterior insula (AIC), left dIPFC, left temporoparietal junction (TPJ) and left cerebellum (Table 2; Figure 2). In line with our hypothesis of higher emotional sensitivity in BPD, simple effects showed higher activity in the AIC for BPD patients compared to NPC for negative stimuli. Interestingly, BPD patients also showed increased activity in the dIPFC and TPJ compared to NPC for negative stimuli.

Resulting clusters were tested for stimulus category specificity. Results showed that, compared to NPC, BPD patients also showed higher activity for positive versus neutral stimuli in the TPJ $\left[F_{1,95}=6.68 ; p=.011\right]$ and at trend level in the $\operatorname{AIC}\left[F_{1,95}=3.85 ; p=.053\right]$. 
Table 2. Resulting clusters for whole-brain RFX ANOVA testing differences in emotional sensitivity and emotion regulation between BPD and NPC regarding negative stimuli.

\begin{tabular}{|c|c|c|c|c|c|c|c|c|}
\hline \multirow{2}{*}{ Brain area } & \multirow{2}{*}{ L/R } & \multirow{2}{*}{ BA } & \multirow{2}{*}{$\begin{array}{c}\text { Cluster size } \\
\mathrm{mm}^{3}\end{array}$} & \multicolumn{3}{|c|}{ Talairach peak voxel } & \multirow{2}{*}{$F$} & \multirow{2}{*}{$p$} \\
\hline & & & & $x$ & $y$ & $z$ & & \\
\hline
\end{tabular}

\section{Emotional Sensitivity}

RFX ANOVA Negative Look versus Neutral Look

\begin{tabular}{|c|c|c|c|c|c|c|c|c|}
\hline Anterior insula & $\mathrm{R}$ & 13 & 1069 & 33 & 17 & 16 & 9.76 & .002 \\
\hline Middle frontal gyrus, dorsolateral & $L$ & 8 & 654 & -30 & 35 & 43 & 12.54 & .001 \\
\hline $\begin{array}{l}\text { Supramarginal gyrus, Temporoparietal } \\
\text { junction }\end{array}$ & L & 40 & 413 & -45 & -43 & 37 & 9.38 & .003 \\
\hline Cerebellum & $\mathrm{L}$ & & 985 & -21 & -34 & -38 & 12.59 & .001 \\
\hline \multicolumn{9}{|l|}{ Emotion Regulation } \\
\hline \multicolumn{9}{|l|}{ RFX ANOVA Negative Safe versus Negative Look } \\
\hline Middle frontal gyrus, dorsolateral & $\mathrm{R}$ & 8 & 831 & 36 & 26 & 37 & 13.96 & $<.001$ \\
\hline Dorsal anterior cingulate cortex & $\mathrm{R}$ & 32 & 1791 & 12 & 17 & 34 & 17.80 & $<.001$ \\
\hline Middle temporal gyrus & $\mathrm{R}$ & 21 & 1596 & 57 & -25 & -11 & 20.47 & $<.001$ \\
\hline Supramarginal gyrus, Inferior parietal lobule & $\mathrm{R}$ & 40 & 1234 & 51 & -52 & 31 & 12.59 & .001 \\
\hline Supramarginal gyrus, Inferior parietal lobule & $L$ & 40 & 2496 & -45 & -43 & 34 & 17.54 & $<.001$ \\
\hline Cerebellum & $\mathrm{R}$ & & 569 & 27 & -37 & -42 & 8.35 & \\
\hline Cerebellum & $L$ & & 1165 & -6 & -73 & -41 & 3.03 & \\
\hline
\end{tabular}

Abbreviations: $\mathrm{L}=$ Left; $\mathrm{R}=$ Right; $\mathrm{BA}=$ Brodmann area.

Thresholded at $p<.005$ and cluster size.

When testing the resulting clusters for diagnosis specificity, a significant difference between BPD and CCP was found in the TPJ $\left[F_{1,77}=4.44 ; p=.038\right]$, suggesting specificity for BPD. No significant differences were found in the AIC and dIPFC for the comparison BPD versus CCP, indicating that activity in these clusters generalizes over personality disorders. Results remained similar when medication was added as a covariate. We post-hoc tested linear and quadratic trends of brain responses in relation to severity of personality psychopathology from NPC to CCP to BPD. We only examined the trends within the clusters which showed a stimulus category or instruction $x$ group interaction. Significant linear trends support the hypothesis that strength of response is a linear function of degree of personality pathology, with cluster-C in-between NPC and BPD; whereas significant quadratic trends support the hypothesis that cluster- $C$ is either similar to NPC, or to BPD. Results supported the linear severity hypothesis, with the CCP scoring in-between BPD and NPC, whereas no evidence for quadratic trends was shown (Table 3A).

Since the whole-brain RFX ANOVA did not result in the hypothesized and previously reported (Herpertz et al., 2001; Koenigsberg et al., 2009b; Schulze et al., 2015) significant differential amyg dala activity, we performed an additional less stringent amygdala region-ofinterest analysis. A main effect of stimulus category was found, in which both BPD and NPC showed increased amygdala activity for negative versus neutral stimuli (Table 3B; Figure 3). 

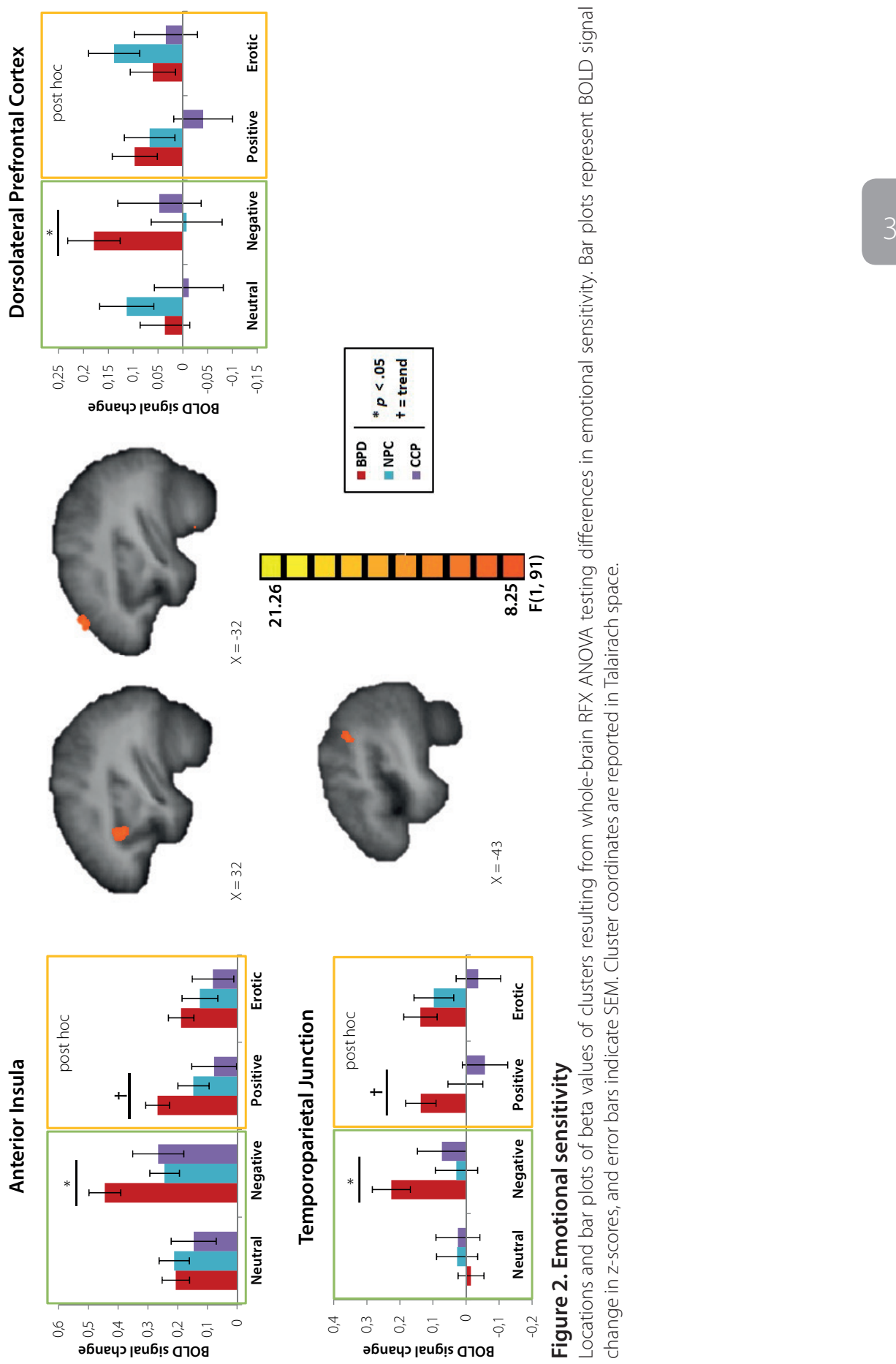
Table 3. Overview of test statistics of additional analyses.

A. Significance levels of linear and quadratic trends of brain responses in relation to severity of personality psychopathology: from NPC to CCP to BPD

\begin{tabular}{|c|c|c|c|c|}
\hline \multirow{2}{*}{ Brain area } & \multicolumn{2}{|c|}{ Look Negative minus Look Neutral } & \multicolumn{2}{|c|}{ Look Positive minus Look Neutral } \\
\hline & Linear & Quadratic & Linear & Quadratic \\
\hline Anterior insula & .002 & .827 & .042 & .330 \\
\hline Dorsolateral prefrontal cortex & .001 & .564 & & \\
\hline \multirow[t]{3}{*}{ Temporoparietal junction } & .002 & .394 & .008 & .050 \\
\hline & \multicolumn{2}{|c|}{ Safe Negative minus Look Negative } & & \\
\hline & Linear & Quadratic & & \\
\hline Dorsolateral prefrontal cortex & .001 & .879 & & \\
\hline Dorsal anterior cingulate cortex & $<.001$ & .809 & & \\
\hline Middle temporal gyrus & $<.001$ & .452 & & \\
\hline Inferior parietal lobule, right & .001 & .307 & & \\
\hline Inferior parietal lobule, left & $<.001$ & .494 & & \\
\hline
\end{tabular}

B. Results of amygdala region-of-interest analyses and separately controlled for potential confounding factors

\begin{tabular}{|c|c|c|c|c|}
\hline & \multicolumn{2}{|c|}{ Left Amygdala } & \multicolumn{2}{|c|}{ Right Amygdala } \\
\hline & $F$ & $p$ & $F$ & $p$ \\
\hline Main effect stimulus & 26.68 & $<.001$ & 30.55 & $<.001$ \\
\hline Interaction stimulus $\times$ group & .73 & .395 & .025 & .876 \\
\hline \multicolumn{5}{|l|}{ controlled for } \\
\hline Time single run 1 & .01 & .922 & 4.19 & .044 \\
\hline Time run 1 to 4 & .49 & .484 & .01 & .935 \\
\hline Dissociation $^{a}$ & .71 & .791 & .54 & .463 \\
\hline Childhood trauma severity ${ }^{b}$ & 1.22 & .272 & .01 & .913 \\
\hline PTSD & 1.73 & .192 & .01 & .925 \\
\hline Depression (BSI subscale) & .24 & .628 & .09 & .767 \\
\hline Medication & .01 & .920 & 1.55 & .217 \\
\hline Lateralization & .14 & .708 & & see left amygdala \\
\hline
\end{tabular}

Abbreviations: PTSD = Posttraumatic stress disorder; BSI = Brief Symptom Inventory.

a Dissociation is mean score of dissociation prior and post scanning.

${ }^{b}$ Childhood trauma severity is the total score of the five subscales: sexual, physical and emotional abuse, and emotional and physical neglect.

However, no significant interaction effects could be detected, even at an uncorrected $p$-value of .05. It seems unlikely that ambiguity of the neutral social stimuli accounts for the failure to detect group differences in amygdala responses (Herpertz et al., 2001), as no evidence was found for increased amygdala responses to the neutral stimuli in BPD (Figure 3). Additional timing analysis, taking the fast habituation of amygdala responses into account (Breiter et al., 1996; Fischer, Furmark, Wik \& Fredrikson, 2000), could not explain 
this null finding. The right amygdala did show a significant stimulus $\times$ group interaction during the first run, simple effect however revealed no group differences but higher activity during negative versus neutral stimuli in both groups. Other potential confounding factors including medication, dissociation, trauma, PTSD, depression (BSI subscale), or lateralization did also not explain this null finding (Table 3B).

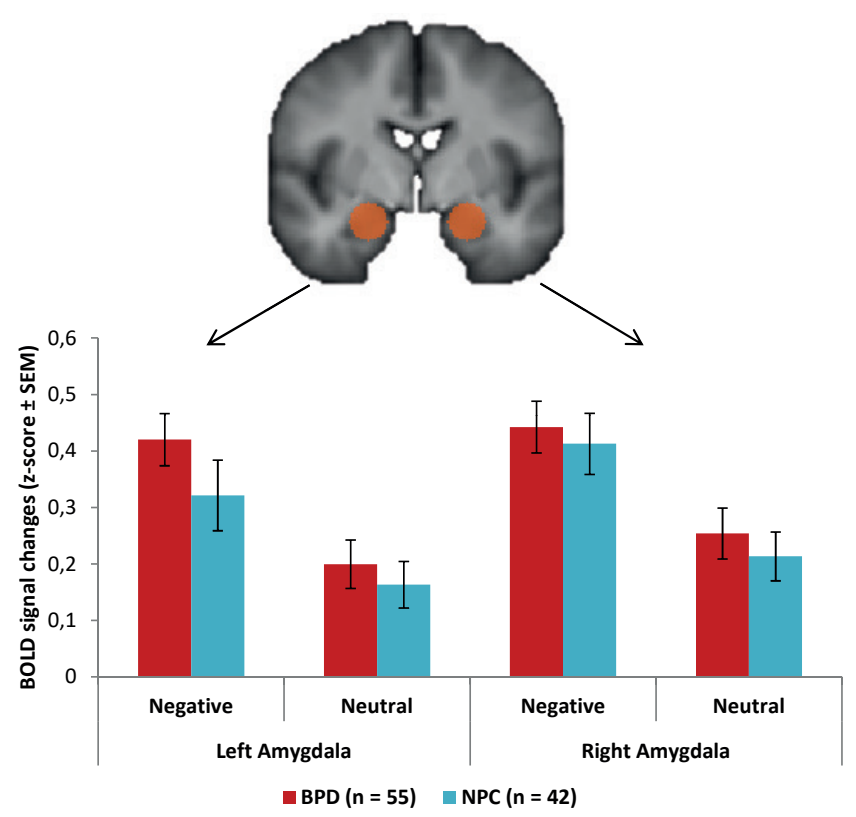

\section{Figure 3. Amygdala seeds}

Location of the left and right amygdala regions-of-interest (Talairach coordinates: $x= \pm 21, y=-5, z=-15$ ) and bar plots of beta values of both amygdala seeds showing a main effect of stimulus category comparing negative versus neutral stimuli.

\section{fMRI results of emotion regulation}

The whole-brain RFX ANOVA F-map: instruction (negative-look vs. negative-safe) x group (BPD vs. NPC) resulted in seven significant clusters, which were identified at the left dACC, left dIPFC, right middle temporal gyrus (MTG), bilateral inferior parietal lobule (IPL) and bilateral cerebellum (Table 2; Figure 4). In line with our hypothesis of impaired emotional regulation abilities in BPD, simple effects showed no significant difference between the safe and look condition in the dIPFC, and decreased activity during the safe versus look condition in the $\mathrm{dACC}$ for BPD patients regarding negative stimuli. Additionally, BPD patients showed less activity during the safe compared to the look condition in the MTG. In contrast, NPC did show significantly more activity in the AACC, dIPFC, MTG and bilateral IPL during the safe versus look condition. 

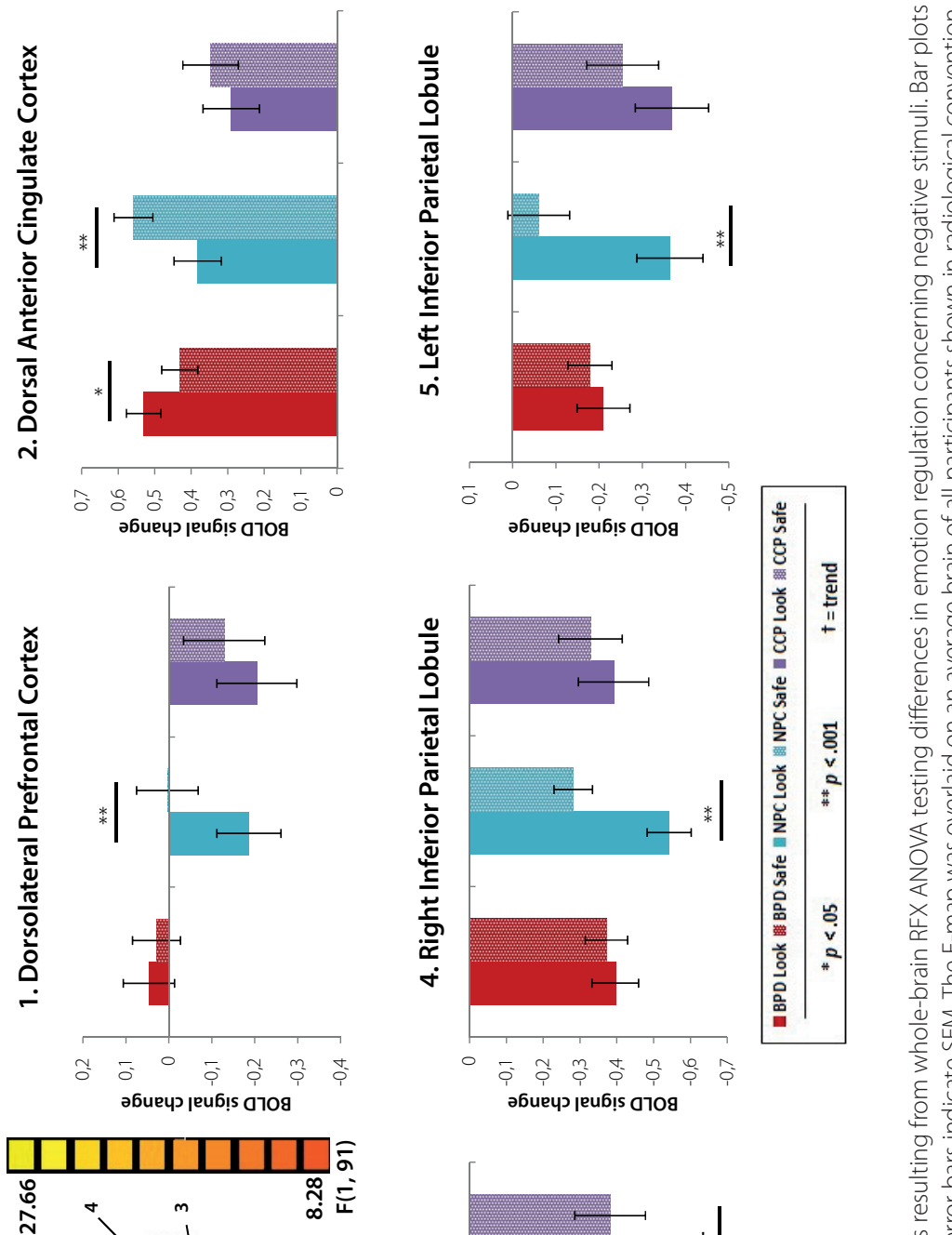
సั
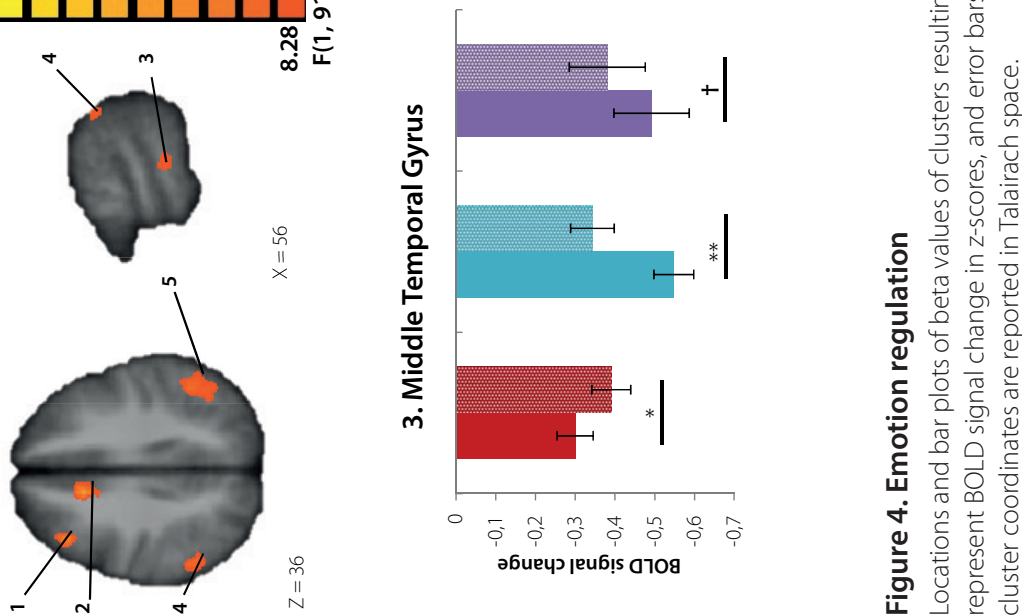
Resulting clusters were tested for stimulus category specificity. No differences were found for the comparison of BPD versus NPC for positive and erotic stimuli in the safe compared to the look condition. This indicates that activity in these clusters is specific for negative stimuli. Results remained the same when corrected for activity during neutral stimuli.

When testing the resulting clusters for diagnosis specificity, a significant interaction for the comparison with CCP was found in the MTG $\left[F_{1,77}=6.66 ; p=.012\right]$, in which CCP showed a significant difference between safe and look, suggesting BPD-diagnosis specificity. Other clusters did not reveal a significant difference between BPD versus CCP, indicating that activity in these clusters is not specific for BPD. When medication was added as a covariate the results remained the same. Again testing post-hoc linear and quadratic trends of brain responses in relation to severity of personality psychopathology, showed a linear relationship over the three groups, with the CCP scoring in-between BPD and NPC (Table 3A).

\section{Discussion}

The aim of the current study was to investigate stimulus category specificity and diagnosis specificity of neural correlates related to the hypothesized increased emotional sensitivity and impaired emotion regulation, underlying emotional instability in BPD. Extending previous research, we added positive and erotic stimuli to the traditional negative and neutral stimuli, and included NPC and CCP groups. In line with our hypothesis, passive viewing of negative stimuli resulted in higher activity in the AIC of BPD compared to NPC. Interestingly, passive viewing of negative stimuli also led to higher activity in the TPJ and dIPFC in BPD. The effect in the AIC and TPJ was not specific for negative stimuli as passive viewing of positive stimuli also resulted in higher activity in BPD compared to NPC. The results of the regulation condition showed higher activity in the AACC, dIPFC, MTG and bilateral IPL when the NPC were instructed to realize to be safe for negative stimuli only, BPD patients did not show this significant increase in activity. As the dACC and dIPFC are important areas for emotion regulation (MacDonald, Cohen, Stenger \& Carter, 2000; Ochsner et al., 2012), this finding supports the hypothesis of impaired emotion regulation in BPD.

Very surprising, previous findings of increased amygdala activity in BPD during the presentation of emotional stimuli (Herpertz et al., 2001; Koenigsberg et al., 2009b; Schulze et al., 2015) were not replicated in the present well-powered study. We therefore run additional analyses, taking dissociation, trauma, posttraumatic stress disorder, depression (BSI subscale), medication, lateralization or fast habituation into account, but could not explain this null finding. Supporting the notion that the amygdala is involved in processing emotional stimuli, we did find a main effect of stimulus category, as both BPD and NPC 
showed increased amygdala activity for negative versus neutral stimuli. However, when differentiating BPD from NPC, our findings rather hint at a key role of the AIC and TPJ in BPD regarding emotional sensitivity. Previous research suggests that the AIC is involved in processing emotional state and affective experience (Craig, 2009), and the TPJ is proposed to be involved in mentalizing (Frith \& Frith, 2006; Shamay-Tsoory, 2011). Maybe BPD patients mainly engage in mentalizing processes when confronted with negative or positive social stimuli, which affect their own emotional bodily state and affective experience.

Also striking, in contrast to previous studies (Koenigsberg et al., 2009a; Schulze et al., 2011; Schulze et al., 2015) current study showed enhanced activity in the dIPFC when BPD patients passively viewed negative stimuli. The dIPFC is commonly associated with effortful control and inhibition processes (MacDonald et al., 2000; Ochsner et al., 2012). Potentially this enhanced activity is an implicit reaction to the increase in bodily state (e.g. arousal) and emotional affect as indicated by the higher activity in the AIC and TPJ. This constant, at least partially failing effort for regulation, might exhaust BPD patients and thus contribute to their emotional instability. Additionally, the IPL within the attentional network is involved in preventing reorientation to unimportant stimuli (Corbetta, Patel \& Shulman, 2008). Therefore, the inability of BPD patients to activate the IPL during emotion regulation might interfere with cognitive control, resulting in difficulties to disengage attention from negative stimuli. It should be noted that reported findings here might differ from previous studies (Koenigsberg et al., 2009a; Schulze et al., 2011) because we applied a new regulation condition.

The specific failure of BPD patients to activate emotion regulation areas when instructed to realize they are safe is in line with the view that BPD is characterized by a lack of safety when experiencing negative emotions, even when reminded (Arntz \& van Genderen, 2009). An important goal of treatment might therefore be to help patients experience negative emotions whilst feeling safe, so that negative emotions no longer need to be avoided and can be processed.

Results regarding stimulus category specificity during emotional sensitivity showed that the AIC and TPJ activity was higher for both negative and positive stimuli, pointing to a general increase in emotional sensitivity in BPD. The emotion regulation results were unique for negative stimuli. This however makes sense, considering that regulation of positive emotions is in most cases undesirable. Finally, we did not find the hypothesized effect for the erotic stimuli. Ratings showed that the valence of the erotic stimuli were more positive as expected, especially in BPD. Our erotic stimuli were related to romance and intimacy and might have been too soft to trigger the expected negative emotional responses related to sexual abuse.

Regarding diagnosis specificity we found a linear relationship in many areas with activity of CCP in-between NPC and BPD. This supports the idea that cluster-C patients display a 
number of features in common with BPD patients, yet weaker, and that the observed effects are more a matter of degree than dichotomous. Considering that social stimuli were used, it makes sense that cluster-C patients show similar response patterns as BPD patients, as both personality disorders show interpersonal difficulties (American Psychiatric Association, 2013). Consequently, it might be useful to manipulate social versus non-social stimuli, to examine whether this dimensionality holds.

Main strengths of the current study are a wider range of stimuli, inclusion of the clinical control group and high statistical power compared to previous studies (van Zutphen et al., 2015). However some limitations need also to be acknowledged. First, we only recruited women which limit the generalizability of our results to men. Second, many BPD patients (69.1\%) were taking psychotropic medication, which might alter individuals'brain responses and is therefore a potentially confounding factor (Delaveau et al., 2011; Ma, 2015). However, we decided not to exclude patients on medication to recruit a representative and severe clinical sample. Within any of the resulting clusters no significant interactions of medication within the BPD group were found, indicating they were robust to medication effects. It should also be acknowledged that it is not clear whether the assumption of requesting patients to be free of medication several weeks before scanning (Koenigsberg et al., 2009a; Koenigsberg et al., 2009b; Schulze et al., 2011) results in a normal brain state at the moment of scanning. Moreover, requiring patients to stop their medication contribute to a sampling bias. Furthermore, for some medication the washed out effect can be several months and long-lasting effects in the brain cannot be ruled out (Hafeman, Chang, Garrett, Sanders \& Phillips, 2012). Third, since we did not monitor eye-movement, we cannot rule out interference of looking away or closing eyes during the task, even though participants were explicitly instructed not to do so. Fourth, we did not consider the menstrual cycle of the participants, which might have affected results, especially with regard to erotic stimuli (Gizewski et al., 2006; Zhu et al., 2010). On the other hand, these effects should be random given the large sample size.

In conclusion, BPD patients showed an elevated response in brain areas important for generation and regulation of emotions when passively viewing negative stimuli compared to NPC. Additionally, BPD patients did not show an increased activity in emotion regulation areas during the safe versus look condition, when presented with negative stimuli, whereas NPC did. In BPD patients, the enhanced responses in brain areas important for emotion generation processing were also present during passive viewing of positive stimuli. Linearity analyses showed evidence of intermediate responses between BPD and NPC of the CCP, implying a dimensional rather than a dichotomous differentiation. 


\section{References}

American Psychiatric Association. (2013). Diagnostic and Statistical Manual of Mental Disorder (5th ed.). Arlington, VA: American Psychiatric Association.

Arntz, A., \& Dreessen, L. (1995). BPD-Klachtenlijst 47 [BPD Checklist]. The Netherlands: Maastricht University.

Arntz, A., van den Hoorn, M., Cornelis, J., Verheul, R., van den Bosch, W. M., \& de Bie, A. J. (2003). Reliability and validity of the borderline personality disorder severity index. Journal of Personality Disorders, 17, 45-59. doi: 10.1521/ pedi.17.1.45.24053

Arntz, A., \& van Genderen, H. (2009). Schema therapy for borderline personality disorder. Chichester: Wiley.

Baczkowski, B. M., van Zutphen, L., Siep, N., Jacob, G. A., Domes, G., Maier, S., . . van de Ven, V. (2016). Deficient amygdala-prefrontal intrinsic connectivity after effortful emotion regulation in borderline personality disorder. European Archives of Psychiatry and Clinical Neuroscience. doi: 10.1007/s00406-016-0760-z

Bradley, M. M., \& Lang, P. J. (1994). Measuring emotion: the Self-Assessment Manikin and the Semantic Differential. Journal of Behavior Therapy and Experimental Psychiatry, 25, 49-59. doi: 10.1016/0005-7916(94)90063-9

Breiter, H. C., Etcoff, N. L., Whalen, P. J., Kennedy, W. A., Rauch, S. L., Buckner, R. L., .. Rosen, B. R. (1996). Response and habituation of the human amygdala during visual processing of facial expression. Neuron, 17, 875-887. doi: 10.1016/S0896-6273(00)80219-6

Corbetta, M., Patel, G., \& Shulman, G. L. (2008). The reorienting system of the human brain: from environment to theory of mind. Neuron, 58, 306-324. doi: 10.1016/j.neuron.2008.04.017

Craig, A. D. (2009). How do you feel--now? The anterior insula and human awareness. Nature Reviews. Neuroscience, 10, 59-70. doi: 10.1038/nrn2555

Delaveau, P., Jabourian, M., Lemogne, C., Guionnet, S., Bergouignan, L., \& Fossati, P. (2011). Brain effects of antidepressants in major depression: a meta-analysis of emotional processing studies. Journal of Affective Disorders, 130, 66-74. doi: 10.1016/j.jad.2010.09.032

Derogatis, L. R. (1993). BSI Brief Symptom Inventory: Administration, Scoring, and Procedure Manual (4th ed.). Minneapolis, MN: National Computer Systems.

First, M. B., Spitzer, R. L., Gibbon, M., \&Williams, J. B. W. (1994). Structured clinical interview for DSM-IV axis I disorders (SCID-I). New York: Biometric Research Department.

First, M. B., Spitzer, R. L., Gibbon, M., Williams, J. B. W., \& Benjamin, L. (1997). Structured clinical interview for DSM-IV axis II personality disorders (SCID-II). New York: Biometric Research Department.

Fischer, H., Furmark, T., Wik, G., \& Fredrikson, M. (2000). Brain representation of habituation to repeated complex visual stimulation studied with PET. NeuroReport, 11, 123-126. doi: 10.1097/00001756-200001170-00024

Forman, S. D., Cohen, J. D., Fitzgerald, M., Eddy, W. F., Mintun, M. A., \& Noll, D. C. (1995). Improved assessment of significant activation in functional magnetic resonance imaging (fMRI): use of a cluster-size threshold. Magnetic Resonance in Medicine, 33, 636-647.

Frith, C. D., \& Frith, U. (2006). The neural basis of mentalizing. Neuron, 50, 531-534. doi: 10.1016/j.neuron.2006.05.001

Giesen-Bloo, J. H., Wachters, L. M., Schouten, E., \& Arntz, A. (2010). The Borderline Personality Disorder Severity IndexIV: psychometric evaluation and dimensional structure. Personality and Individual Differences, 49, 136-141. doi: DOl 10.1016/j.paid.2010.03.023

Gizewski, E. R., Krause, E., Karama, S., Baars, A., Senf, W., \& Forsting, M. (2006). There are differences in cerebral activation between females in distinct menstrual phases during viewing of erotic stimuli: a fMRI study. Experimental Brain Research, 174, 101-108.

Goebel, R., Esposito, F., \& Formisano, E. (2006). Analysis of functional image analysis contest (FIAC) data with brainvoyager QX: From single-subject to cortically aligned group general linear model analysis and selforganizing group independent component analysis. Human Brain Mapping, 27, 392-401. doi: 10.1002/ hbm.20249

Hafeman, D. M., Chang, K. D., Garrett, A. S., Sanders, E. M., \& Phillips, M. L. (2012). Effects of medication on neuroimaging findings in bipolar disorder: an updated review. Bipolar Disorders, 14, 375-410. doi: 10.1111/j.13995618.2012.01023.x

Herpertz, S. C., Dietrich, T. M., Wenning, B., Krings, T., Erberich, S. G., Willmes, K., . . Sass, H. (2001). Evidence of abnormal amygdala functioning in borderline personality disorder: a functional MRI study. Biological Psychiatry, 50, 292-298. doi: 10.1016/S0006-3223(01)01075-7 
Jacob, G. A., Arntz, A., Domes, G., Reiss, N., \& Siep, N. (2011). Positive erotic picture stimuli for emotion research in heterosexual females. Psychiatry Research, 190, 348-351. doi: 10.1016/j.psychres.2011.05.044

Koenigsberg, H. W., Fan, J., Ochsner, K. N., Liu, X., Guise, K. G., Pizzarello, S., .. Siever, L. J. (2009a). Neural correlates of the use of psychological distancing to regulate responses to negative social cues: a study of patients with borderline personality disorder. Biological Psychiatry, 66, 854-863. doi: 10.1016/j.biopsych.2009.06.010

Koenigsberg, H. W., Siever, L. J., Lee, H., Pizzarello, S., New, A. S., Goodman, M., .. Prohovnik, I. (2009b). Neural correlates of emotion processing in borderline personality disorder. Psychiatry Research, 172, 192-199. doi: 10.1016/j.pscychresns.2008.07.010

Krause-Utz, A., Winter, D., Niedtfeld, I., \& Schmahl, C. (2014). The latest neuroimaging findings in borderline personality disorder. Current Psychiatry Reports, 16, 438. doi: 10.1007/s11920-014-0438-z

Kroger, C., Vonau, M., Kliem, S., Roepke, S., Kosfelder, J., \& Arntz, A. (2013). Psychometric properties of the German version of the borderline personality disorder severity index--version IV. Psychopathology, 46, 396-403. doi: $10.1159 / 000345404$

Lang, P. J., Bradley, M. M., \& Cuthbert, B. N. (1997). International Affective Picture System (IAPS): Instruction Manual and Affective Ratings. Gainesville, FL: Center of Research in Psychophysiology, University of Florida.

Leichsenring, F., Leibing, E., Kruse, J., New, A. S., \& Leweke, F. (2011). Borderline personality disorder. Lancet, 377, 7484. doi: 10.1016/S0140-6736(10)61422-5

Lenzenweger, M. F., Lane, M. C., Loranger, A. W., \& Kessler, R. C. (2007). DSM-IV personality disorders in the National Comorbidity Survey Replication. Biological Psychiatry, 62, 553-564. doi: 10.1016/j.biopsych.2006.09.019

Lieberman, M. D., \& Cunningham, W. A. (2009). Type I and Type II error concerns in fMRI research: re-balancing the scale. Social Cognitive and Affective Neuroscience, 4, 423-428. doi: 10.1093/scan/nsp052

Linehan, M. M. (1993). Cognitive-Behavioral Treatment of Borderline Personality Disorder. New York: Guilford Press.

Lobbestael, J., Arntz, A., Harkema-Schouten, P., \& Bernstein, D. (2009). Development and psychometric evaluation of a new assessment method for childhood maltreatment experiences: the interview for traumatic events in childhood (ITEC). Child Abuse \& Neglect, 33, 505-517. doi: 10.1016/j.chiabu.2009.03.002

Ma, Y. (2015). Neuropsychological mechanism underlying antidepressant effect: a systematic meta-analysis. Molecular Psychiatry, 20, 311-319. doi: 10.1038/mp.2014.24

MacDonald, A. W., 3rd, Cohen, J. D., Stenger, V. A., \& Carter, C. S. (2000). Dissociating the role of the dorsolateral prefrontal and anterior cingulate cortex in cognitive control. Science, 288, 1835-1838. doi: 10.1126/ science.288.5472.1835

Mauchnik, J., \& Schmahl, C. (2010). The latest neuroimaging findings in borderline personality disorder. Current Psychiatry Reports, 12, 46-55. doi: 10.1007/s11920-009-0089-7

Morawetz, C., Holz, P., Lange, C., Baudewig, J., Weniger, G., Irle, E., \& Dechent, P. (2008). Improved functional mapping of the human amygdala using a standard functional magnetic resonance imaging sequence with simple modifications. Magnetic Resonance Imaging, 26, 45-53. doi: 10.1016/j.mri.2007.04.014

Ochsner, K. N., Bunge, S. A., Gross, J. J., \& Gabrieli, J. D. (2002). Rethinking feelings: an fMRI study of the cognitive regulation of emotion. Journal of Cognitive Neuroscience, 14, 1215-1229. doi: 10.1162/089892902760807212

Ochsner, K. N., Silvers, J. A., \& Buhle, J.T. (2012). Functional imaging studies of emotion regulation: a synthetic review and evolving model of the cognitive control of emotion. Annals of the New York Academy of Sciences, 1251, E124. doi: 10.1111/j.1749-6632.2012.06751.x

Schulze, L., Domes, G., Kruger, A., Berger, C., Fleischer, M., Prehn, K., ... Herpertz, S. C. (2011). Neuronal correlates of cognitive reappraisal in borderline patients with affective instability. Biological Psychiatry, 69, 564-573. doi: 10.1016/j.biopsych.2010.10.025

Schulze, L., Schmahl, C., \& Niedtfeld, I. (2015). Neural correlates of disturbed emotion processing in borderline personality disorder: a multimodal meta-analysis. Biological Psychiatry. doi: 10.1016/j.biopsych.2015.03.027

Sempertegui, G. A., Karreman, A., Arntz, A., \& Bekker, M. H. (2013). Schema therapy for borderline personality disorder: a comprehensive review of its empirical foundations, effectiveness and implementation possibilities. Clinical Psychology Review, 33, 426-447. doi: 10.1016/j.cpr.2012.11.006

Shamay-Tsoory, S. G. (2011). The neural bases for empathy. The Neuroscientist, 17, 18-24. doi: $10.1177 / 1073858410379268$ 
Stiglmayr, C. E., Shapiro, D. A., Stieglitz, R. D., Limberger, M. F., \& Bohus, M. (2001). Experience of aversive tension and dissociation in female patients with borderline personality disorder -- a controlled study. Journal of Psychiatric Research, 35, 111-118. doi: 10.1016/S0022-3956(01)00012-7

Trull, T. J., Jahng, S., Tomko, R. L., Wood, P. K., \& Sher, K. J. (2010). Revised NESARC personality disorder diagnoses: gender, prevalence, and comorbidity with substance dependence disorders. Journal of Personality Disorders, 24, 412-426. doi: 10.1521/pedi.2010.24.4.412

van Asselt, A. D., Dirksen, C. D., Arntz, A., \& Severens, J. L. (2007). The cost of borderline personality disorder: societal cost of illness in BPD-patients. European Psychiatry, 22, 354-361. doi: 10.1016/j.eurpsy.2007.04.001

van Zutphen, L., Siep, N., Jacob, G. A., Goebel, R., \& Arntz, A. (2015). Emotional sensitivity, emotion regulation and impulsivity in borderline personality disorder: A critical review of fMRI studies. Neuroscience and Biobehavioral Reviews, 51C, 64-76. doi: 10.1016/j.neubiorev.2015.01.001

van Zutphen, L., Siep, N., Jacob, G. A., Domes, G., Sprenger, A., Willenburg, B., ... Arntz, A. (n.d.). Impulse control under emotion processing: an fMRI investigation in borderline personality disorder compared to non-patients and cluster-C personality disorder patients. Submitted

Wetzelaer, P., Farrell, J., Evers, S., Jacob, G. A., Lee, C. W., Brand, O., ... Arntz, A. (2014). Design of an international multicentre RCT on group schema therapy for borderline personality disorder. BMC Psychiatry, 14, 319. doi: 10.1186/s12888-014-0319-3

Whittle, S., Yucel, M., Yap, M. B., \& Allen, N. B. (2011). Sex differences in the neural correlates of emotion: evidence from neuroimaging. Biological Psychology, 87, 319-333. doi: 10.1016/j.biopsycho.2011.05.003

Zanarini, M. C., Yong, L., Frankenburg, F. R., Hennen, J., Reich, D. B., Marino, M. F., \& Vujanovic, A. A. (2002). Severity of reported childhood sexual abuse and its relationship to severity of borderline psychopathology and psychosocial impairment among borderline inpatients. The Journal of Nervous and Mental Disease, 190, 381 387. doi: 10.1097/00005053-200206000-00006

Zhu, X., Wang, X., Parkinson, C., Cai, C., Gao, S., \& Hu, P. (2010). Brain activation evoked by erotic films varies with different menstrual phases: an fMRI study. Behavioural Brain Research, 206, 279-285. doi: 10.1016/j. bbr.2009.09.027 


\section{Supplementary Methods}

\section{Measures}

\section{Brief Symptom Inventory (BSI)}

The BSI is a brief psychological self-report inventory of general symptoms of psychopathology during the past week (Derogatis, 1993). It is a short alternative for the Symptom Checklist-90-R from which it was developed. It contains 53 items divided over nine dimensions: somatization, obsession-compulsion, interpersonal sensitivity, depressive mood, anxiety, hostility, phobic anxiety, paranoid ideation and psychoticism. Answers are scored on a 5-point Likert scale, ranging from 0 (not at all) to 4 (extremely). Scores of the dimensions are calculated by summing the values for the items divided by the number of items within the subscales. The total score measures the level of symptomatology, which is the sum of the nine dimensions plus the four additional items divided by total number of items. The internal consistency showed a Cronbach's a of .96 for the total instrument and ranged between .71 and .85 for its subscales (De Beurs \& Zitman, 2006; Derogatis, 1993).To distinguish patients from non-patients a cutoff score of 70 is suggested (De Beurs, 2004).

\section{BPD Checklist}

The BPD Checklist is a self-report questionnaire used to assess the burden of BPD symptoms as experienced during the last month (Arntz \& Dreessen, 1995). It consists of 47 items based on the nine dimensions of BPD in DSM-IV. Items must be rated on 5-point Likert scale, ranging from 1 (not at all) to 5 (extremely). Next to the total sumscore, also the scores for the nine subscales can be calculated. Scores above 100 signify BPD pathology and therefore is indicative as cutoff for inclusion criteria. When control patients showed an elevated score, an extra check with the SCID-II BPD section was done.

\section{Interview for Traumatic Events in Childhood (ITEC)}

The ITEC is a retrospective, semi-structured interview to measure childhood maltreatment prior the age of 18 including sexual (12 items), physical (13 items) and emotional abuse (9 items), and emotional (6 items) and physical neglect (15 items) (Lobbestael, Arntz, HarkemaSchouten \& Bernstein, 2009). For each item the participant experienced maltreatment, follow-up questions are used to gather more detailed information about the perpetrator(s), age of onset, frequency, duration of the trauma and the impact on the victim in the past and in the present. This information was used to calculate a severity score between 0 and 1 , such that the score increased with the severity of the event itself, the closeness of the perpetrator, the younger age of onset, the longer duration of the event, and the higher impact on the victim. For each subscales the severity scores for the events are summed, the higher this score, the more severe the maltreatment. Internal consistencies of these scales 
were moderate to excellent, with Cronbach's a varying between .58 and .89 with a mean of .79 (Lobbestael et al., 2009). In addition to the victimization scales, similar scales were created for witnessing the various forms of maltreatment. In current study only the victim scales were reported. On average the administration time is about 30 minutes but can take up to one hour in case of multiple maltreatments.

\section{Dissociation and Anxiety}

Present state dissociative experiences were assessed using four items of the DissociationTension-Scale (Stiglmayr, Shapiro, Stieglitz, Limberger \& Bohus, 2001), containing derealization and changes in perception of one's body, hearing and pain. Additionally two items concerning the level of anxiety and the level of nervousness were added. Responses were indicated on a visual analogue scale, ranging from 0 not at all to 100 extreme. The dissociation score was the averaged across the four dissociation items. Internal consistencies of the dissociation score proved to be good in current sample, with a Cronbach's $a$ of .83 concerning dissociation before scanning and a Cronbach's a of .86 concerning dissociation after scanning.

\section{Self-Assessment Manikin Scale}

The pictures shown during the scanning session were qualitatively assessed for valence and arousal using the Self-Assessment Manikin Scale. The Self-Assessment Manikin Scale consists of a series of human-like figures to measures the affective reaction of a person to stimuli (Bradley \& Lang, 1994). Intensity of valence and arousal were both rated on a 9-point scale, with for valence 1 being extremely unpleasant and 9 being extremely pleasant, and for arousal 1 being most calm and 9 being most aroused.

\section{Wechsler Adult Intelligence Scale}

IQ was estimated by means of four subtests of the Wechsler Adult Intelligence Scale, including two verbal (i.e. Vocabulary and Similarities) and two nonverbal tests (i.e. Block design and Matrix reasoning). Together these subtests correlate strongly with general intellectual ability (Schrimsher, O'Bryant, O'Jile \& Sutker, 2008). IQ was estimated based on the optimized regression equation: $39.05+(1.54 *$ comprehension score $)+(1.64 *$ matrix reasoning score $)+(1.48 *$ similarities score $)+(.98 *$ picture arrangement score $)$. If the WAIS score was not available (BPD patients $n=11, \operatorname{NPC} n=3$ and CCP $n=1$ ) an estimation of the IQ was made based on the education level, using the regression equation from our present sample per group; IQ BPD patients $=85.932+(3.360 *$ ISCED code), IQ NPC $=85.996+(3.659$

* ISCED code) and IQ CCP $=81.529+(4.226 *$ ISCED code). These regression equations explained respectively $22 \%, 31 \%$ and $36 \%$ of the variance in IQ. Level of education of both 
the Dutch and German educational systems were transformed into the International Standard Classification of Education (ISCED).

\section{Borderline Personality Disorder Severity Index (BPDSI)}

The BPDSI is a semi-structured clinical interview assessing frequency and severity of BPD manifestations (Arntz et al., 2003; Giesen-Bloo, Wachters, Schouten \& Arntz, 2010; Kroger et al., 2013). The 70-items reflect the nine BPD criteria described in the DSM-IV. For each item the frequency of the last three months is rated on an 11-point scale, ranging from 0 (never) to 10 (daily). The scores on the subscales provide information on the severity of each of the DSM-IV dimensions, derived by averaging the items scores. The total score is the sum of the nine dimensions scores, ranging from 0 to 90, with an internal consistency of Cronbach's $a=.93$ and subscales ranged between .41 and .83 (Arntz et al., 2003). A total score of 20 distinguishes BPD from other personality disorders (Giesen-Bloo et al., 2010). 


\section{Supplementary Results}

\section{Behavioral data}

To examine whether the experienced emotion during scanning (Table S1) after each trial depended on the stimulus category or differed between groups an instruction (look vs. safe) $x$ stimulus (negative, positive, erotic vs. neutral) x group (BPD, NPC vs. CCP) repeated measures ANOVA was performed. No significant three-way interaction was shown. Besides the significant instruction $x$ stimulus interaction $\left[F_{3.114}=3.94 ; p=.010\right.$ : see main text $]$ the stimulus $\times$ group interaction $\left[F_{6,230}=2.56 ; p=.020\right]$ was significant. Post hoc tests revealed that BPD patients reported significantly stronger emotions during erotic $\left[t_{93}=-3.19 ; p=\right.$ .002] and neutral $\left[t_{93}=-3.28 ; p=.001\right]$ stimuli than NPC. BPD patients did not significantly differ from CCP, who did not differ from NPC.

To examine whether the arousal and valence ratings of the stimulus evaluations after scanning (Table S1) differed between groups two stimulus (negative, positive, erotic vs. neutral) $x$ group (BPD, NPC vs. CCP) repeated measures ANOVAs were performed, one involving arousal ratings and the other involving valence ratings. The stimulus evaluations after scanning showed besides a main effect of arousal $\left[F_{3,80}=74.38 ; p<.001\right.$; see main text $]$ a significant valence $x$ group interaction $\left[F_{6,162}=2.52 ; p=.024\right]$. Post hoc tests showed that BPD patients rated the erotic $\left[t_{61}=-2.96 ; p=.004\right]$, neutral $\left[t_{61}=-3.85 ; p<.001\right]$ and positive $\left[t_{61}=3.19 ; p=.002\right]$ stimuli significantly less pleasant than NPC. CCP rated neutral stimuli significantly less pleasant than NPC $\left[t_{54}=2.59 ; p=.012\right]$. Negative stimuli did not differ in valence rating across groups.

\section{Exploratory analyses of BPD patients compared to both control groups when viewing positive stimuli versus neutral stimuli}

The whole brain random effects ANOVA F-map: stimulus (positive-look vs. neutral-look) $x$ group (BPD vs. NPC) resulted in five clusters, which were anatomically identified at the left amygdala, left middle occipital gyrus and the cerebellum (Table S2). Simple effects showed higher activity in the amygdala and middle occipital gyrus for NPC when presented with positive versus neutral stimuli. No differences were found for the comparison of BPD with $\mathrm{CCP}$, also when corrected for the significant difference of medication between BPD and CCP.

\section{Exploratory analyses of BPD patients compared to both control groups when viewing erotic stimuli versus neutral stimuli}

The whole brain random effects ANOVA F-map: stimulus (erotic-look vs. neutral-look) $x$ group (BPD vs. NPC) resulted in two clusters, which were anatomically identified at the medial prefrontal cortex and temporal pole (Table S2). In the medial prefrontal cortex, simple effects showed higher activity in the NPC for erotic versus neutral stimuli. Furthermore, no 
differences were found for the comparison of BPD and CCP. In the temporal pole, simple effects showed less activity for BPD patients compared to NPC when presented with neutral stimuli. Additionally, a significant difference was found between BPD and CCP $\left[F_{1,77}=5.09 ; p\right.$ $=.007]$. These results did hold when corrected for medication.

\section{Exploratory analyses of BPD patients compared to both control groups during the safe versus look conditions when presented with positive stimuli}

The whole brain random effects ANOVA F-map: stimulus (positive-safe vs. positive-look) $x$ group (BPD vs. NPC) resulted in two clusters, which were anatomically identified at the anterior prefrontal cortex and pons (Table S2). Relative to NPC, simple effects showed in BPD higher activity during the safe compared to the look condition in the anterior prefrontal cortex. Moreover, as a significant difference was found for the comparison with CCP $\left[F_{1,77}=\right.$ 8.89; $p=.004]$, these results point to be specific for BPD. Results remained the same when controlled for activity during neutral stimuli and medication.

Table S1. Ratings of experienced emotion during scanning and stimulus evaluation after scanning of the three groups.

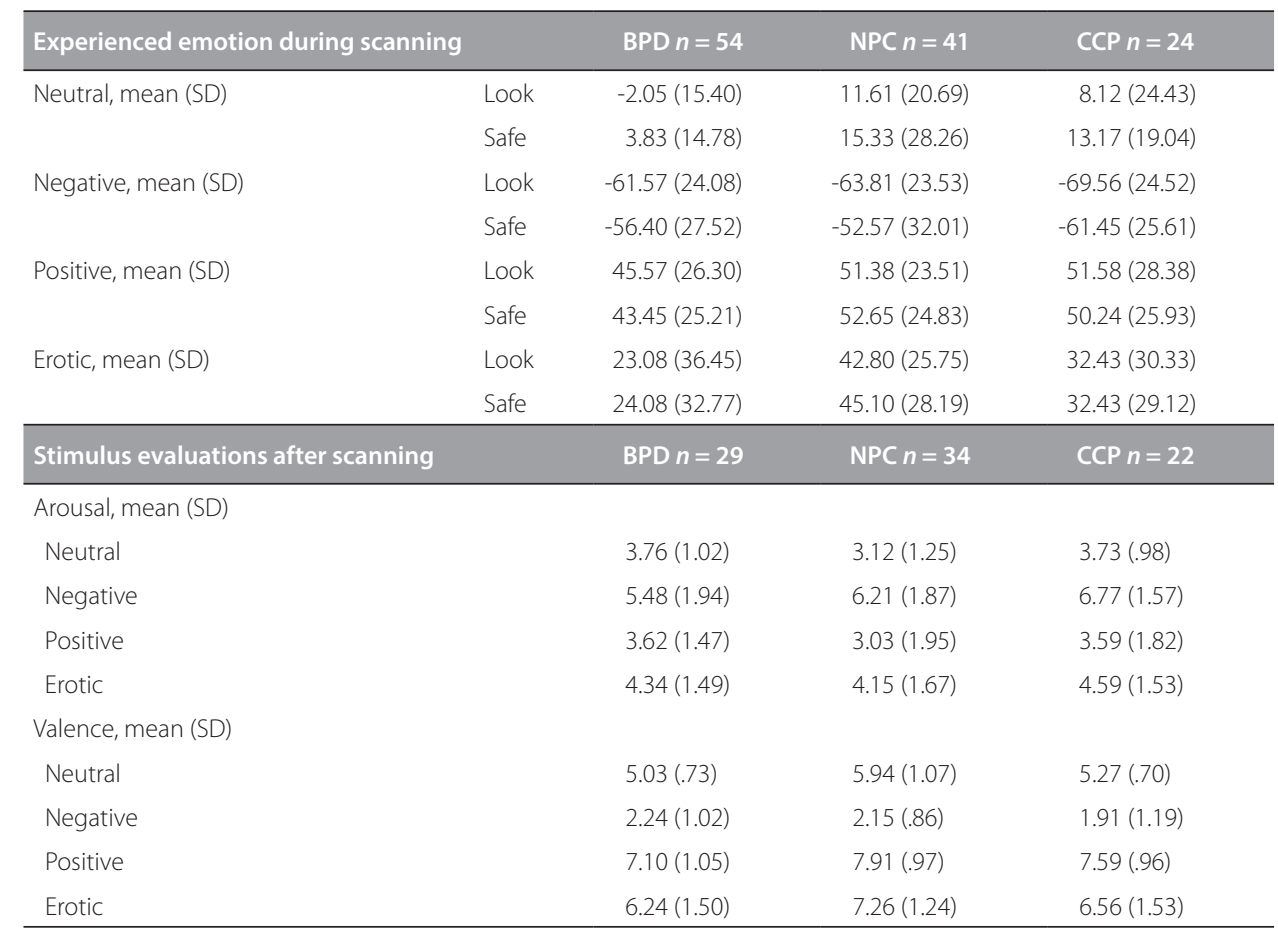


Table S2. Significant brain areas of between BPD and NPC comparison for additional whole brain random effects ANOVAs.

\begin{tabular}{|c|c|c|c|c|c|c|c|c|}
\hline \multirow{2}{*}{ Brain area } & \multirow{2}{*}{ L/R } & \multirow{2}{*}{ BA } & \multirow{2}{*}{$\begin{array}{c}\text { Cluster size } \\
\mathrm{mm}^{3}\end{array}$} & \multicolumn{3}{|c|}{ Talairach peak voxel } & \multirow{2}{*}{$F$} & \multirow{2}{*}{$p$} \\
\hline & & & & $x$ & $y$ & $z$ & & \\
\hline
\end{tabular}

\section{Emotional Sensitivity}

Look Positive versus Look Neutral, cluster threshold $=15$

\begin{tabular}{|c|c|c|c|c|c|c|c|c|}
\hline Amygdala & L & & 430 & -24 & -10 & -11 & 11.20 & .001 \\
\hline Middle occipital gyrus & $\mathrm{R}$ & 19 & 658 & 18 & -85 & 16 & 12.69 & .001 \\
\hline Cerebellum & $\mathrm{R}$ & & 1795 & 12 & -88 & -30 & 15.14 & $<.001$ \\
\hline Cerebellum & $\mathrm{R}$ & & 459 & 12 & -64 & -41 & 8.05 & .006 \\
\hline Cerebellum & $\mathrm{R}$ & & 854 & -12 & -73 & -17 & 14.11 & $<.001$ \\
\hline \multicolumn{9}{|c|}{ Look Erotic versus Look Neutral, cluster threshold $=11$} \\
\hline Medial frontal gyrus & $L$ & 10 & 408 & -3 & 59 & 7 & 14.24 & $<.001$ \\
\hline Superior temporal gyrus, temporal pole & $\mathrm{R}$ & 38 & 411 & 18 & 11 & -36 & 9.41 & .003 \\
\hline
\end{tabular}

\section{Emotion Regulation}

Safe Positive versus Look Positive, cluster threshold $=10$

\begin{tabular}{|c|c|c|c|c|c|c|c|}
\hline Middle frontal gyrus, anterior & $\mathrm{R}$ & 352 & 45 & 53 & 4 & 9.30 & .003 \\
\hline Pons & $\mathrm{R}$ & 504 & 12 & -16 & -20 & 12.46 & .001 \\
\hline
\end{tabular}

Abbreviations: $L=$ Left; $R=$ Right; $B A=$ Brodmann area. Thresholded at $p<.005$ and cluster size. 


\section{References}

Arntz, A., \& Dreessen, L. (1995). BPD-Klachtenlijst 47 [BPD Checklist]. The Netherlands: Maastricht University.

Arntz, A., van den Hoorn, M., Cornelis, J., Verheul, R., van den Bosch, W. M., \& de Bie, A. J. (2003). Reliability and validity of the borderline personality disorder severity index. Journal of Personality Disorders, 17, 45-59. doi: 10.1521/ pedi.17.1.45.24053

Bradley, M. M., \& Lang, P. J. (1994). Measuring emotion: the Self-Assessment Manikin and the Semantic Differential. Journal of Behavior Therapy and Experimental Psychiatry, 25, 49-59. doi: 10.1016/0005-7916(94)90063-9

De Beurs, E. (2004). Handleiding bij de Brief Symptom Inventory (BSI). Leiden: Pits Publishers.

De Beurs, E., \& Zitman, F. G. (2006). De Brief Symptom Inventory (BSI): De betrouwbaarheid en validiteit van een handzaam alternatief voor de SCL-90 [The Brief Symptom Inventory (BSI): The reliability and validity of a brief alternative of the SCL-90]. Maandblad Geestelijke Volksgezondheid, 61, 120-141.

Derogatis, L. R. (1993). BSI Brief Symptom Inventory: Administration, Scoring, and Procedure Manual (4th ed.). Minneapolis, MN: National Computer Systems.

Giesen-Bloo, J. H., Wachters, L. M., Schouten, E., \& Arntz, A. (2010). The Borderline Personality Disorder Severity IndexIV: psychometric evaluation and dimensional structure. Personality and Individual Differences, 49, 136-141. doi: DOl 10.1016/j.paid.2010.03.023

Kroger, C., Vonau, M., Kliem, S., Roepke, S., Kosfelder, J., \& Arntz, A. (2013). Psychometric properties of the German version of the borderline personality disorder severity index--version IV. Psychopathology, 46, 396-403. doi: $10.1159 / 000345404$

Lobbestael, J., Arntz, A., Harkema-Schouten, P., \& Bernstein, D. (2009). Development and psychometric evaluation of a new assessment method for childhood maltreatment experiences: the interview for traumatic events in childhood (ITEC). Child Abuse \& Neglect, 33, 505-517. doi: 10.1016/j.chiabu.2009.03.002

Schrimsher, G. W., O'Bryant, S. E., O'Jile, J. R., \& Sutker, P. B. (2008). Comparison of tetradic WAIS-III short forms in predicting full scale IQ scores in neuropsychiatric clinic settings. Journal of Psychopathology and Behavioral Assessment, 30, 235-240. doi: DOI 10.1007/s10862-007-9066-9

Stiglmayr, C. E., Shapiro, D. A., Stieglitz, R. D., Limberger, M. F., \& Bohus, M. (2001). Experience of aversive tension and dissociation in female patients with borderline personality disorder -- a controlled study. Journal of Psychiatric Research, 35, 111-118. doi: 10.1016/S0022-3956(01)00012-7 


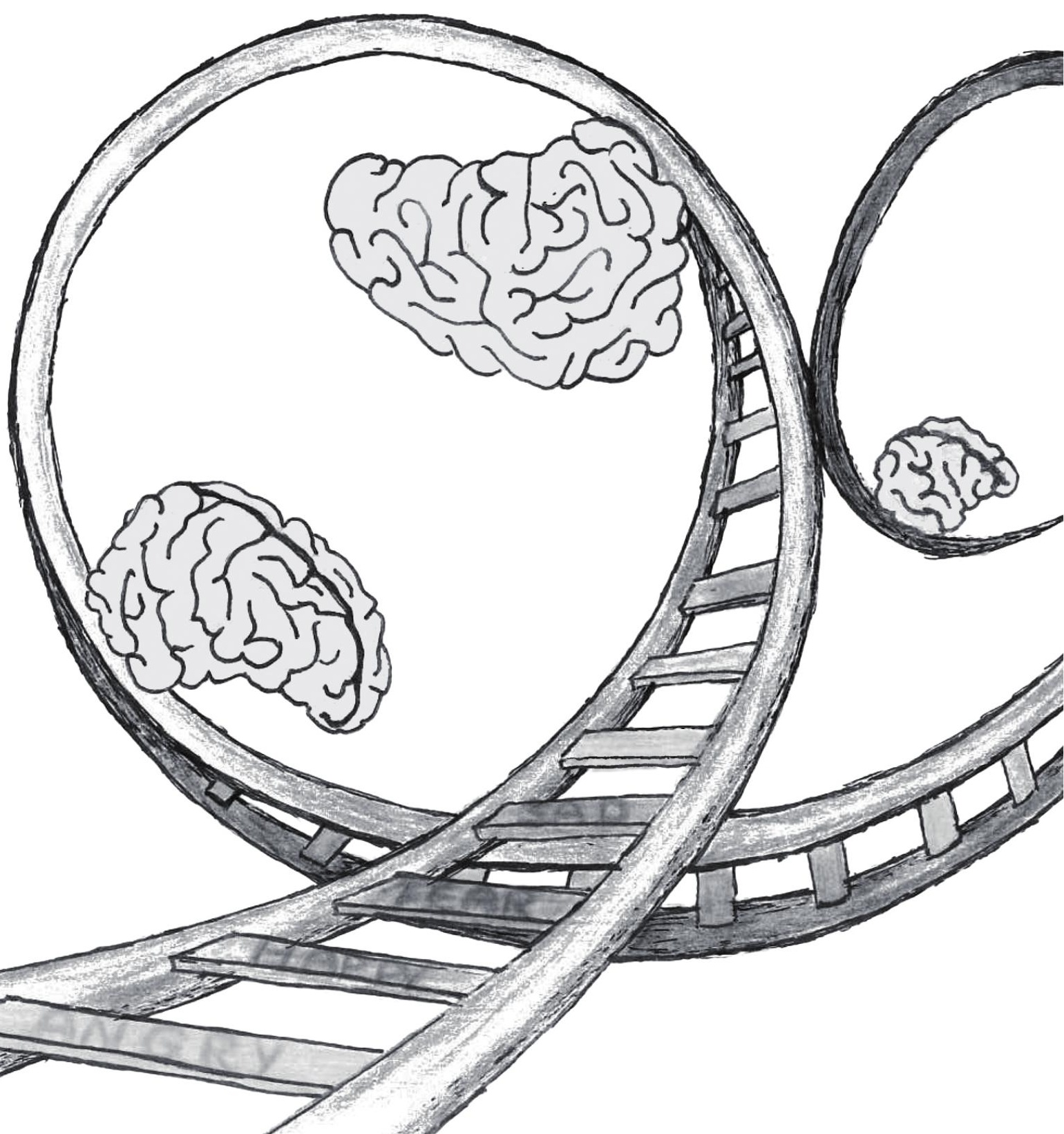




\section{Impulse control under emotion processing: an $\mathrm{fMRI}$ investigation in borderline personality disorder compared to non-patients and cluster-c personality disorder patients}

\section{This chapter has been submitted as:}

Van Zutphen, L., Siep, N., Jacob, G.A., Domes, G., Sprenger, A., Willenborg, B., Goebel, R., Tüscher O. \& Arntz, A. (n.d.). Impulse control under emotion processing: an fMRI investigation of borderline personality disorder compared to non-patients and cluster-c personality disorder patients. submitted 


\begin{abstract}

\section{Background}

Impulsivity is a characteristic syndromal and neurobehavioral feature of borderline personality disorder (BPD). Research suggests an important interaction between high negative emotions and low behavioral inhibition in BPD. However, knowledge about the generalizability across stimulus categories and diagnosis specificity is limited. We investigated neural correlates of hypothesized impaired response inhibition of BPD patients to negative, positive and erotic stimuli, by comparing them to non-patients and cluster-C personality disorder patients.
\end{abstract}

\title{
Methods
}

During fMRI scanning, 53 BPD patients, 34 non-patients and 21 cluster-C personality disorder patients completed an affective go/no-go task, including social pictures.

\section{Results}

BPD patients showed more omission errors than non-patients, independent of the stimulus category. Furthermore, BPD patients showed higher activity in the frontal eye fields when inhibiting negative stimuli, while non-patients showed less activity in the inferior parietal lobule when inhibiting negative stimuli. In general, cluster-C patients showed activity that was in-between BPD and non-patients.

\section{Conclusions}

When inhibiting emotional stimuli, BPD patients showed an altered brain activity in the inferior parietal lobe and frontal eye fields. BPD patients showed a general responsivity across stimulus categories in the inferior parietal lobe, whereas effects in the frontal eye fields were specific for negative stimuli. Results of diagnosis specificity support a dimensional rather than a categorical differentiation between BPD and cluster- $C$ patients during inhibition of social emotional stimuli. Supported by behavioral results, BPD patients showed no deficiencies in emotionally modulated response inhibition per se but the present findings rather hint at attentional difficulties for emotional information.

Keywords: BPD, Response inhibition, Impulsivity, Emotion, fMRI, Neuroimaging 


\section{Introduction}

One of the hallmarks of borderline personality disorder (BPD) is impulsivity; commonly associated with self-injury, anger outbursts, substance abuse, unprotected sex, excessive spending, reckless driving, and uncontrolled eating (American Psychiatric Association, 2013; Sebastian, Jacob, Lieb \& Tüscher, 2013a). Impulsivity is a broad concept often defined as a pattern of behavioral disinhibition, including a predisposition of rapidly and unplanned responses without considering the consequences (Moeller, Barratt, Dougherty, Schmitz \& Swann, 2001). Hence, impulsivity has often been related to response inhibition difficulties.

Behavioral data resulting from BPD studies investigating the hypothesized impaired response inhibition, using go/no-go tasks, are inconsistent (Sebastian et al., 2013a). Some studies did not report differences between BPD and non-patients (NPC) (Jacob et al., 2010; Vollm et al., 2004), while others showed that BPD patients make significantly more commission errors, supporting that they are indeed worse in response inhibition (Leyton et al., 2001; Mortensen, Rasmussen \& Håberg, 2010; Rentrop et al., 2008). Neuroimaging studies examining response inhibition in BPD are also inconclusive. An fMRI study in BPD patients compared to NPC showed a negative correlation between the number of commission errors and activation in the medial frontal gyrus, anterior cingulate cortex (ACC), temporal gyrus and striatum (Leyton et al., 2001). Two other fMRI studies did not show any differences in brain activity between BPD and NPC (Mortensen et al., 2010; van Eijk et al., 2015).

In response to the conflicting results, it has been suggested that BPD patients act impulsive especially in negative emotional conditions (Domes et al., 2006; Jacob et al., 2010; Sebastian et al., 2013a). This interaction between negative emotions and impulsivity has been examined in two fMRI studies using go/no-go tasks (Jacob et al., 2013; Silbersweig et al., 2007). Silbersweig et al. (2007) reported a decreased ventromedial prefrontal cortex (VmPFC) and increased lateral orbitofrontal cortex (OFC) and dorsolateral PFC (dIPFC) activity in BPD compared to NPC during response inhibition of negative words. Additionally, in BPD a negative correlation between impulsivity scores and VmPFC activity was shown (Silbersweig et al., 2007). Another study (Jacob et al., 2013) examined response inhibition after anger induction and found in BPD compared to NPC a decreased inferior frontal cortex (IFC) and increased nucleus subthalamicus activity. Since no significant group differences were shown at behavioral level, the authors suggested a compensatory mechanism involving the subthalamic nucleus for the missing IFC activity (Jacob et al., 2013). Together, negative emotions might interfere with behavioral inhibition and underlie impulsivity in BPD, represented by heightened activity in brain areas responding to emotions accompanied by decreased activity in brain areas that control behavioral impulses.

The first aim of the present international, multicenter study is to examine neurocircuits involved in response inhibition in BPD. Both previous studies exclusively reported negative with neutral stimuli contrasts. Accordingly it remains unclear whether impaired response 
inhibition solely involves negative emotions, or whether it also holds for positive emotions, pointing to a general impaired response inhibition. Furthermore, given that history of sexual traumatization is often reported in BPD (Zanarini et al., 2002), erotic content might trigger intense emotional reactions leading to impulsive behavior. Consequently, erotic stimuli were included. Additionally, because of high rates of comorbid Axis I and II disorders (McGlashan et al., 2000; Zanarini et al., 1998a, 1998b; Zimmerman \& Mattia, 1999) within BPD it remains uncertain whether findings are diagnosis specific or characteristic of psychopathology in general. By adding a clinical control group (CCP) the second aim of the present study is to examine BPD-specificity of impulsivity. Motivated by prior work it was hypothesized that BPD patients have more problems with inhibiting their response under emotional states compared to both control groups, related to higher activity in emotion-related brain areas (amygdala, hippocampus, ventral striatum, anterior insula, posterior cingulate cortex (PCC)) (Goldstein et al., 2007; Ochsner, Silvers \& Buhle, 2012; Shafritz, Collins \& Blumberg, 2006) and dysregulated activity in areas related to response inhibition (IFC/ventrolateral PFC (vIPFC), dorsal ACC, dIPFC, vmPFC, inferior parietal lobe (IPL), pre-supplementary motor area, thalamus, dorsal striatum, nucleus subthalamicus) (Goldstein et al., 2007; Sebastian et al., 2013b; Shafritz et al., 2006; Simmonds, Pekar \& Mostofsky, 2008; Swick, Ashley \& Turken, 2011).

\section{Materials and Methods}

\section{Participants}

The present study complements our recent study that examined stimulus category specificity and diagnosis specificity of neural correlates of emotional regulation in BPD (van Zutphen et al., submitted). Fifty-nine BPD patients, 41 NPC and 29 CCP underwent the second scanning session, of which 53 BPD patients, 34 NPC and 21 CCP met scanning and clinical criteria and were left for the analyses.

Participants were recruited from two sites in the Netherlands (Maastricht, Heerlen) and three sites in Germany (Freiburg, Hamburg, Lübeck). Patients were recruited from the mental health clinics at local sites. NPC were recruited among the general population at each site via postings and personal contacts. Participants had to be hetero- or bisexual females, aged 18-65, and have sufficient understanding of the language at the local site. We additionally assessed the Brief Symptom Inventory (Derogatis, 1993), BPD checklist (Arntz \& Dreessen, 1995) and Interview for Trauma Events in Childhood (Lobbestael, Arntz, Harkema-Schouten \& Bernstein, 2009). For demographic and diagnostic variables of all groups see Table 1. No significant group differences were shown for age, handedness and IQ. See supplemental materials for participant recruitment and measurements.

Before onset of the study, written informed consent was obtained. Participants received a small financial remuneration. The study was approved by the local medical ethical committees (Wetzelaer et al., 2014). 
Table 1. Demographic and diagnostic variables of the three groups.

\begin{tabular}{|c|c|c|c|c|c|}
\hline & \multirow{2}{*}{$\begin{array}{c}\text { BPD } \\
(n=53) \\
\end{array}$} & \multirow{2}{*}{$\begin{array}{c}\text { NPC } \\
(n=34)\end{array}$} & \multirow{2}{*}{$\begin{array}{c}\text { CCP } \\
(n=21)\end{array}$} & \multicolumn{2}{|c|}{ Test Statistics } \\
\hline & & & & $F$ & $p$ \\
\hline Age, years, mean (SD) & $31.02(8.77)$ & $29.44(11.31)$ & $30.10(10.40)$ & .269 & .765 \\
\hline Education levela, No. (\%) & & & & $4.21^{b}$ & .122 \\
\hline Level 1 & $12(22.6)$ & $7(20.6)$ & $3(14.3)$ & & \\
\hline Level 2 & $8(15.1)$ & $2(5.9)$ & $4(19.0)$ & & \\
\hline Level 3 & $15(28.3)$ & $4(11.8)$ & $6(28.6)$ & & \\
\hline Level 4 & $2(3.8)$ & $2(5.9)$ & $3(14.3)$ & & \\
\hline Level 5 & $13(24.5)$ & $14(41.2)$ & $3(14.3)$ & & \\
\hline Level 6 & $3(5.7)$ & $5(14.7)$ & $2(9.5)$ & & \\
\hline Estimated IQ', mean (SD) & $96.43(9.88)$ & $98.82(11.05)$ & $98.22(9.62)$ & .629 & .535 \\
\hline Handedness, No. L/R/M & $3 / 46 / 3$ & 1/33/- & $-/ 21 /-$ & $4.87^{\mathrm{d}}$ & .301 \\
\hline BSI, mean (SD), total & $1.74(.56)$ & $.14(.15)$ & $1.09(.45)$ & 132.30 & $<.001^{\mathrm{e}}$ \\
\hline BPD checklist, mean (SD), total & $119.92(25.03)$ & $51.26(6.46)$ & $74.25(17.74)$ & 134.70 & $<.001^{\mathrm{f}}$ \\
\hline ITEC, mean (SD) & & & & 8.53 & $<.001^{9}$ \\
\hline Sexual abuse & $9.02(9.05)$ & $.11(.39)$ & $2.26(5.43)$ & 17.45 & $<.001$ \\
\hline Physical abuse & $17.26(11.79)$ & $1.64(3.58)$ & $7.34(10.83)$ & 24.63 & $<.001$ \\
\hline Emotional abuse & $20.24(8.78)$ & $2.47(3.48)$ & $13.45(8.56)$ & 51.17 & $<.001$ \\
\hline Emotional neglect & $11.31(6.75)$ & $.80(1.94)$ & $6.36(6.67)$ & 31.23 & $<.001$ \\
\hline Physical neglect & $10.24(9.14)$ & $.96(2.95)$ & $4.51(6.89)$ & 15.43 & $<.001$ \\
\hline Dissociation, mean (SD) & & & & 7.04 & $<.001^{\mathrm{h}}$ \\
\hline prior scanning & $19.09(19.89)$ & $2.37(2.49)$ & $7.10(10.63)$ & 14.10 & $<.001$ \\
\hline post scanning & $26.20(23.15)$ & $4.96(7.55)$ & $10.15(15.66)$ & 15.26 & $<.001$ \\
\hline Anxiety, mean (SD) & & & & 6.92 & $<.001^{i}$ \\
\hline prior scanning & $26.10(25.55)$ & $3.18(5.46)$ & $15.14(20.93)$ & 12.98 & $<.001$ \\
\hline post scanning & $18.94(24.40)$ & $1.88(1.93)$ & $7.24(9.48)$ & 10.28 & $<.001$ \\
\hline Nervousness, mean (SD) & & & & 7.16 & $<.001^{\mathrm{j}}$ \\
\hline prior scanning & $32.75(27.31)$ & $5.26(9.63)$ & $19.05(22.09)$ & 15.79 & $<.001$ \\
\hline post scanning & $20.77(24.79)$ & $2.88(3.55)$ & $11.52(16.79)$ & 9.19 & $<.001$ \\
\hline Axis I disorders, No. (\%) & & & & & $p^{k}$ \\
\hline Major depressive disorder & $47(88.7)$ & & $13(61.9)$ & & .008 \\
\hline Dysthymic & $4(7.5)$ & & $1(4.8)$ & & .667 \\
\hline Bipolar type II & $1(1.9)$ & & - & & .526 \\
\hline Generalized anxiety disorder & $1(1.9)$ & & - & & .526 \\
\hline Panic disorder with agoraphobia & $7(13.2)$ & & $1(4.8)$ & & .292 \\
\hline Panic disorder & $7(13.2)$ & & $3(14.3)$ & & .903 \\
\hline Agoraphobia & $3(5.7)$ & & - & & .266 \\
\hline Specific phobia & $10(18.9)$ & & - & & .032 \\
\hline Social phobia & $18(34.0)$ & & $5(23.8)$ & & .395 \\
\hline Obsessive compulsive disorder & $8(15.1)$ & & $2(9.5)$ & & .527 \\
\hline Posttraumatic stress disorder & $20(37.7)$ & & $3(14.3)$ & & .049 \\
\hline Somatoform disorder & $5(9.4)$ & & $4(19.0)$ & & .254 \\
\hline
\end{tabular}


Table 1. Demographic and diagnostic variables of the three groups. (Continued)

\begin{tabular}{|c|c|c|c|c|c|}
\hline & \multirow{2}{*}{$\begin{array}{c}\text { BPD } \\
(n=53)\end{array}$} & \multirow{2}{*}{$\begin{array}{c}\text { NPC } \\
(n=34)\end{array}$} & \multirow{2}{*}{$\begin{array}{c}\text { CCP } \\
(n=21)\end{array}$} & \multicolumn{2}{|c|}{ Test Statistics } \\
\hline & & & & $F$ & $p$ \\
\hline Eating disorders & $20(37.7)$ & & $7(33.3)$ & & .723 \\
\hline Substance abuse & $26(49.1)$ & & $1(4.8)$ & & $<.001$ \\
\hline Intermitted explosive disorder & $1(1.9)$ & & - & & .526 \\
\hline \multicolumn{6}{|l|}{ Axis II disorders, No. (\%) } \\
\hline Avoidant PD & $26(50.9)$ & & $15(71.4)$ & & .081 \\
\hline Dependent PD & $9(17.0)$ & & $2(9.5)$ & & .416 \\
\hline Obsessive compulsive PD & $10(18.9)$ & & $6(28.6)$ & & .361 \\
\hline Passive aggressive PD & $4(7.5)$ & & - & & .196 \\
\hline Depressive PD & $15(28.3)$ & & $2(9.5)$ & & .083 \\
\hline Paranoid PD & $15(28.3)$ & & - & & .006 \\
\hline Schizotypal PD & $1(1.9)$ & & - & & .526 \\
\hline Schizoid PD & $1(1.9)$ & & - & & .526 \\
\hline \multicolumn{6}{|l|}{ Medication, No. (\%) } \\
\hline Antidepressants & $36(67.9)$ & & $9(42.9)$ & & .046 \\
\hline Antipsychotics & $8(15.1)$ & & - & & .059 \\
\hline Hypnotics & $3(5.7)$ & & - & & .266 \\
\hline Mood Stabilizers & $1(1.9)$ & & - & & .526 \\
\hline
\end{tabular}

Abbreviations: $\mathrm{L}=$ Left; $\mathrm{R}=$ Right; $\mathrm{M}=$ Mixed; BSI = Brief Symptom Inventory; BPD checklist = Borderline checklist; ITEC = Interview Traumatic Events Childhood; PD = Personality Disorder.

a Level of education of both the Dutch and German educational systems were translated into the International Standard Classification of Education (ISCED), in current study six levels of education were divided ranging from lower secondary school to Master's degree.

${ }^{\mathrm{b}}$ Value is based on Kruskal-Wallis.

cAssessed with four subtasks of the WAIS.

${ }^{d}$ Value is based on Chi-square, data of one BPD patient not available.

e All three groups significantly differed from each other $(p<.001)$.

${ }^{f}$ All three groups significantly differed from each other $(p<.001)$, data of one CCP not available.

${ }^{9}$ MANOVA and ANOVAs showed significant group effects over traumas. BPD patients experienced significantly more trauma compared to both control groups regarding sexual abuse (vs. NPC $p<.001$; vs. CCP $p=.001$ ), physical abuse (vs. NPC $p<.001$; vs. CCP $p=.001$ ) and physical neglect (vs. NPC $p<.001$; vs. CCP $p=.012$ ). The three groups significantly differed from each other concerning emotional abuse (BPD vs. NPC $p<.001$; BPD vs. CCP $p=.003$; NPC vs. CCP $p<.001$ ) and emotional neglect (BPD vs. NPC $p<.001$; BPD vs. CCP $p=.005$; NPC vs. CCP $p=.004$ ), with BPD patients experiencing the most trauma, followed by the CCP and the NPC experienced the least trauma. Data of five NPC and one CCP not available. ${ }^{h}$ MANOVA and ANOVAs showed significant group effects over dissociation. BPD patients dissociated significantly more prior and post scanning compared to both control groups (prior scanning: BPD vs. NPC $p<.001$, BPD vs. CCP $p=.008$; post scanning: BPD vs. NPC $p<.001$, BPD vs. NPC $p=.003$ ). Data of six BPD patients and one CCP not available.

'MANOVA and ANOVAs showed significant group effects over anxiety. BPD patients were more anxious compared to NPC prior scanning, and more anxious compared to both control groups post scanning (prior scanning: BPD vs. NPC $p<.001$; post scanning: BPD vs. NPC $p=.001$, BPD vs. CCP $p=.033$ ). Data of five BPD patients not available.

${ }^{j}$ MANOVA and ANOVAs showed significant group effects over nervousness. BPD patients were more anxious compared to NPC prior and post scanning (prior scanning: BPD vs. NPC $p<.001$; post scanning: BPD vs. NPC $p<.001$ ). Data of five BPD patients not available.

${ }^{k}$ Value is based on Chi-square. 


\section{Go/No-go task}

Participants underwent scanning while they performed a visual affective go/no-go task (Figure 1; adapted from Silbersweig et al. (2007)). Neutral, negative, positive and erotic pictures were presented in a blue or yellow frame. Participants were instructed to perform as fast and correctly as possible a right-index-finger button-press when the picture was framed within a blue square (go-trials), while they had to inhibit this motor response when the picture was framed within a yellow square (nogo-trials). Button-press responses and reaction times were recorded. Pictures were selected from the International Affective Picture System (Lang, Bradley \& Cuthbert, 1997) and additional erotic pictures from Jacob et al. (Jacob, Arntz, Domes, Reiss \& Siep, 2011). Only pictures with a social content were selected since BPD patients are particularly responsive to interpersonal cues (Koenigsberg et al., 2009).

A
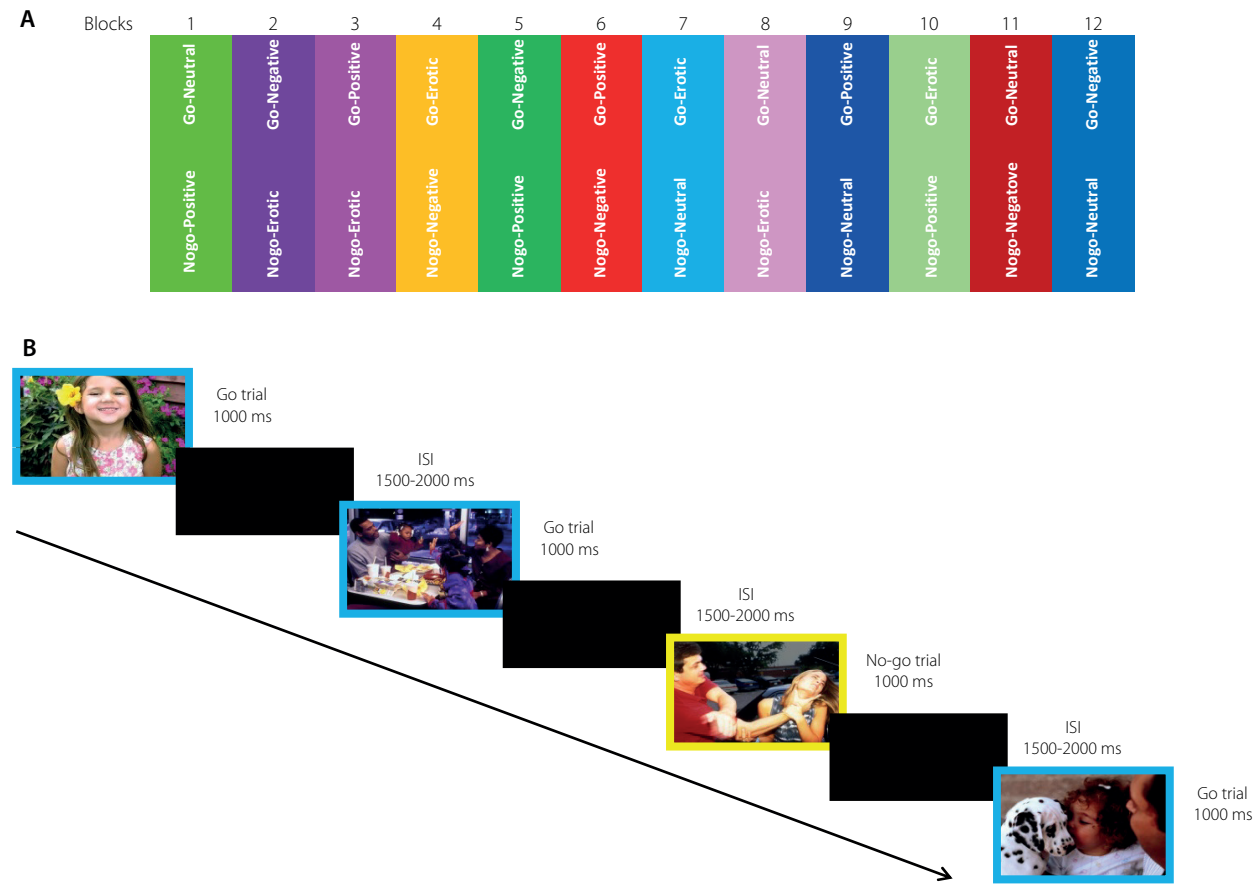

\section{Figure 1. Task design}

Panel A depicts the order of the blocks and set go/nogo-combinations. Panel B shows a block in which positive pictures were combined with go-trials and negative pictures with nogo-trials. Stimuli were presented for 1000 msec and followed by a variable inter-stimulus-interval (ISI) of 1500-2000 msec. Participants had to make a button press for the go trials (blue square), while they had to inhibit this motor response for the no-go trials (yellow square). 
The task consisted of four runs with 12 blocks per run. Each block consisted of 16 randomized trials of two stimulus categories with $25 \%$ nogo-trials and $75 \%$ go-trials. Due to the differentiation of go and no-go stimuli, and because of the four different affective picture categories, 12 go/no-go combinations were set. All combinations of picture categories were used so each nogo-trial is controlled for any other picture category (Figure 1A). In order to induce a prepotent motor response each block started with a minimum of four go-trials.

\section{Data analyses}

Images were obtained on 3-Tesla scanners. All preprocessing and statistical analyses were performed with BrainVoyager 2.6 (Brain Innovation, Maastricht, The Netherlands). Procedure, scanning parameters and preprocessing are described in the supplementary material. To model the hemodynamic nogo-response the first four go-trials of each block (to induce the prepotent response tendency) were considered as separate go-blocks, with the next 12 mixed go/nogo-trials constituting a nogo-block. This resulted in 24 block types, 12 for each no-go distinguished by the stimulus category of the go, and 12 for each go differentiated by the stimulus category of the no-go. Since we were interested in response inhibition under emotional processing in general and not in specific go/no-go combinations the applied general linear model included eight predictors collapsed irrespective of the specific stimulus category of the go's or no-go's; denoting go-neutral, go-negative, go-positive, goerotic, nogo-neutral, nogo-negative, nogo-positive, nogo-erotic. Subsequently, six motion parameters were added as confound predictors.

Differences in brain activity between BPD and NPC during response inhibition of negative stimuli were first used to define the clusters, in a second step we looked for the effects of positive and erotic stimuli, and CCP. Individual statistical parametric maps were generated for the nogo-negative versus nogo-neutral contrast. These contrast images were entered into group-level analyses, including group (BPD, NPC) and site (Maastricht, Freiburg, Lubeck) as between-factors. Next, a whole-brain random-effects (RFX) ANOVA was carried out including nogo-stimulus (nogo-negative vs. nogo-neutral) x group (BPD vs. NPC). The resulting F-maps were thresholded at $p<.005$ and cluster-size, being 12 voxels, to balance type-I and II errors (Lieberman \& Cunninham, 2009). The minimal cluster-size was determined by a cluster-level estimation plugin implemented in BrainVoyager, which performs a clusterlevel correction of multiple comparisons using a Monte Carlo simulation-based approach (1000 iterations: Forman et al., 1995). For each cluster beta values per predictor, per run, of each participant individually were extracted and exported to SPSS 21 (IBM Corporation, New York). To investigate how BPD-specific our results were, mean betas per cluster of the CCP were additionally extracted from the clusters and used in post-hoc comparisons. The same strategy was applied to examine the BPD response-uniqueness to negative stimuli; mean betas per cluster of the positive and erotic stimuli were extracted. 
Subsequently, to explore differences in brain activity during response inhibition of positive or erotic stimuli the following interactions were performed: nogo-stimulus (nogopositive vs. nogo-neutral) x group (BPD vs. NPC), and nogo-stimulus (nogo-erotic vs. nogoneutral) $x$ group (BPD vs. NPC).

Linear mixed model (LMM) analyses were used for detailed analyses, to control for 1) the response of the go-trials within the blocks by using the beta values of the go's as a time-dependent covariate, and 2) the unbalanced design (as go's and no-go's of the same stimulus category were never combined in one block). First-order autoregression was chosen as covariance structure for the repeated part, including run and order of the block within the run, as this led to the best fitting models. Backwards stepwise deletion was used to eliminate non-significant variables and interactions. The fixed part contained dummies of the nogo-stimulus categories, group and their interactions, and the response on the go's and order of the block as running covariates. A random intercept and slope for run, with covariance components as covariance structure, of each participant were included as this resulted in a better fit of the models.

\section{Results}

\section{Behavioral data}

To examine group differences the number of commission errors (go reaction in nogo-trial), omission errors (no-go reaction in go-trial), and reaction times of commissions or hits (Table 2) four LMM-analyses were performed. No significant interactions were shown. The number of commissions, reaction times of commissions and reaction times of hits showed a main effect of stimulus category $\left(F_{3,3040.74}=4.13, p=.006 ; F_{3,3050.71}=4.94, p=.002 ; F_{3,2640.30}=6.97, p\right.$ $<.001$, respectively). Post-hoc tests revealed more commissions $(B=-.065, \mathrm{SE}=.019, p=$ .003 ) and longer reaction times of these commissions $(B=-31.12, \mathrm{SE}=8.11, p=.001)$ for negative versus erotic stimuli. The reaction time of the positive hits were longer versus the neutral $(B=-10.05, S E=2.24, p<.001)$ and erotic hits $(B=-6.44, S E=2.39, p=.043)$. The number of omissions showed a main effect of group $\left(F_{2,70.18}=4.87, p=.015\right)$, in which BPD patients made more omissions than NPC $(B=.137, \mathrm{SE}=.047, p=.011)$ and marginally more omissions than CCP ( $B=.119, \mathrm{SE}=.051, p=.069)$ (Neural correlates of group differences regarding response inhibition are reported in the supplementary materials).

\section{Functional MRI results}

The RFX ANOVA F-map: nogo-stimulus (nogo-negative vs. nogo-neutral) $x$ group (BPD vs. NPC) resulted in the left IPL and left frontal eye fields (FEF) (Table 3A). LMM-analysis showed higher activity in the FEF when inhibiting negative stimuli in BPD, whereas NPC showed less activity in the IPL when inhibiting negative stimuli (Figure 2). Furthermore, BPD showed marginally less activity in the IPL compared to NPC for neutral stimuli. 
Table 2. Behavioral data of the three groups during the go/no-go task for the neutral, negative, positive and erotic stimuli.

\begin{tabular}{lccc} 
& BPD $(n=27)$ & NPC $(n=26)$ & CCP $(n=19)$ \\
\hline Omissions, mean (SD) & & & \\
Neutral & $2.26(3.74)$ & $.58(1.39)$ & $.89(1.24)$ \\
Negative & $2.22(3.76)$ & $.50(1.14)$ & $.89(1.47)$ \\
Positive & $1.89(3.14)$ & $.73(1.43)$ & $.26(.45)$ \\
Erotic & $2.67(3.88)$ & $.38(1.06)$ & $.79(1.08)$ \\
Commissions, mean (SD) & & & \\
Neutral & $1.93(1.70)$ & $2.35(2.91)$ & $2.37(2.85)$ \\
Negative & $2.59(1.91)$ & $2.31(2.29)$ & $2.95(3.46)$ \\
Positive & $2.15(2.01)$ & $1.96(1.99)$ & $2.95(2.70)$ \\
Erotic & $1.74(1.78)$ & $1.85(2.01)$ & $1.95(2.46)$ \\
RT Commissions, & & & \\
mean (SD), msec & & $287.19(207.08)$ & $260.00(206.29)$ \\
Neutral & $388.46(229.93)$ & $391.28(240.02)$ & $323.00(231.45)$ \\
Negative & $430.41(182.16)$ & $271.32(195.52)$ & $393.53(137.11)$ \\
Positive & $323.91(211.99)$ & $278.94(220.05)$ & $273.18(216.89)$ \\
Erotic & $310.28(259.13)$ & & \\
RT Hits, mean (SD), msec & & $457.47(59.05)$ & $472.37(55.72)$ \\
Neutral & $487.02(58.62)$ & $448.62(63.15)$ & $469.99(58.93)$ \\
Negative & $479.88(57.43)$ & $445.06(59.59)$ & $457.46(59.61)$ \\
Positive & $477.12(56.79)$ & $451.13(54.36)$ & $466.22(57.11)$ \\
Erotic & $483.01(55.13)$ &
\end{tabular}

Results showed a significant interaction for positive and erotic stimuli in the IPL (Table 3A). Pairwise comparisons showed higher activity when inhibiting neutral versus erotic stimuli in NPC (Figure 2). The comparison BPD versus CCP did not show significant differences. In addition to the go-response, a dummy of the go-stimulus category (to equalize the gostimulus category across the nogo-negative and nogo-neutral blocks) was added. Post-hoc sensitivity analyses with go-stimulus category as additional covariate showed similar results. Since we did not detect differences between BPD and CCP, we post-hoc tested linear and quadratic trends of brain responses in relation to severity of personality psychopathology from NPC to CCP to BPD. Both clusters support a linear relationship for the significant nogostimulus $x$ group interactions over the three groups, with the CCP scoring in-between the BPD and NPC (Table 3B; Figure 2).

Each cluster was checked for confounding effect of medication. LMM-analyses within BPD showed no significant [stimulus $x$ medication (medicated vs. non-medicated)] interactions in the clusters. Moreover the results remained the same for the BPD versus CCP comparison when medication was added to the LMM-analyses including both groups. 


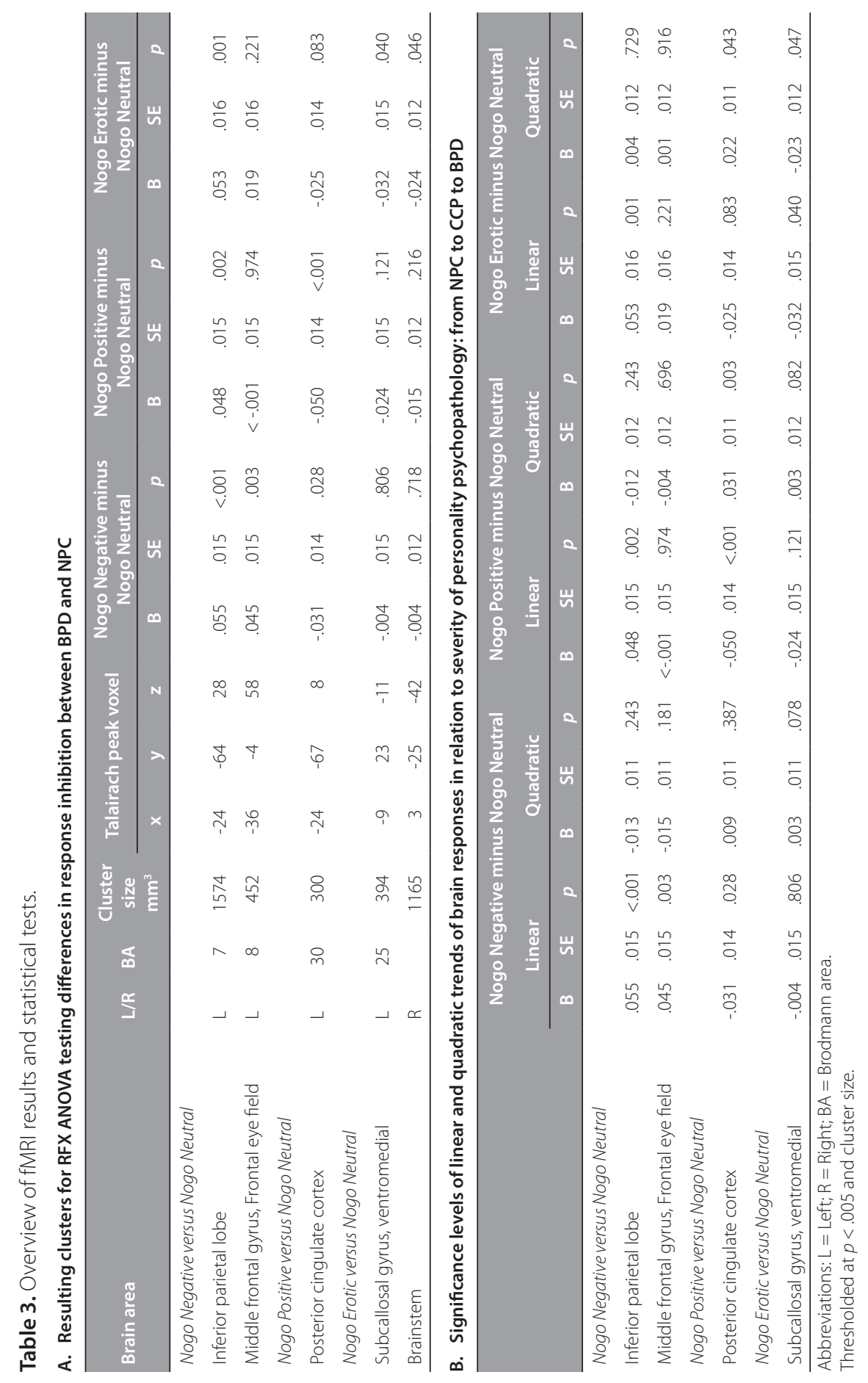




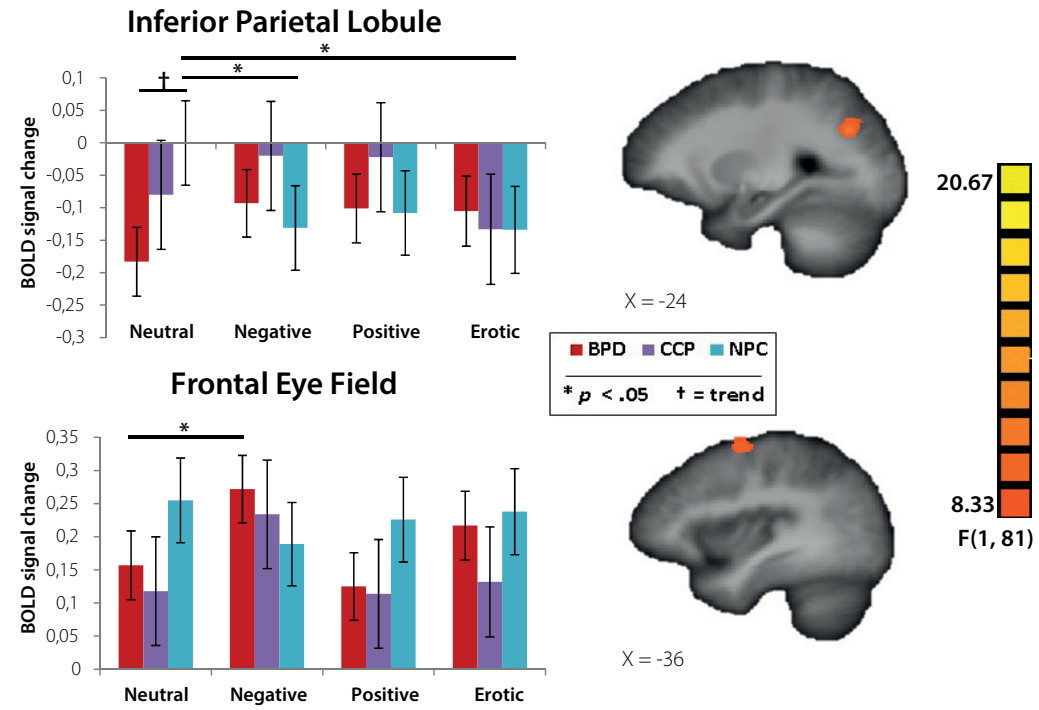

Figure 2. Response inhibition

Locations of clusters resulting from the whole-brain RFX ANOVA testing differences in response inhibition. Cluster coordinates are reported in Talairach space. Bar plots represent mean estimates and standard error of beta values based on linear mixed model analyses.

\section{Exploratory analyses of BPD patients compared to both control groups when inhibiting positive and erotic stimuli versus neutral stimuli}

To explore the differences in brain activity between BPD and NPC specifically during response inhibition of positive or erotic stimuli the following two interactions were conducted: nogostimulus (nogo-positive vs. nogo-neutral) x group (BPD vs. NPC), and nogo-stimulus (nogoerotic vs. nogo-neutral) $\times$ group (BPD vs. NPC). The minimal cluster-size thresholds of the resulting F-maps were 11 and 12 voxels, respectively. The interaction regarding response inhibition of positive stimuli revealed the left PCC (Table 3A). LMM-analysis showed less activity in BPD compared to NPC when inhibiting positive versus neutral stimuli. No differences were found for the comparison of BPD and CCP. The interaction concerning response inhibition of erotic stimuli exhibited the left vmPFC and right brainstem (Table $3 \mathrm{~A}$ ). A significant difference was found between BPD and CCP in the VMPFC, BPD showed less activity compared to CCP for erotic stimuli $(B=-.050, \mathrm{SE}=.018, p=.006)$. Post-hoc sensitivity analyses with go-stimulus category as additional covariate showed similar results for the PCC and vmPFC. Furthermore, analyses of linear or quadratic relationship of brain responses in relation to severity of personality psychopathology showed no exclusive evidence in both clusters (Table 3B). Finally, LMM-analyses within BPD showed no significant stimulus $x$ medication interactions, and the results of the comparison of BPD with CCP did also hold when corrected for medication. 


\section{Discussion}

The aim of the current fMRI study was to investigate stimulus category specificity and diagnosis specificity of response inhibition under emotional processing in BPD. Elaborating on previous research, we used an affective go/no-go paradigm and added positive and erotic stimuli to the traditional negative and neutral stimuli, and compared BPD to nonpatient and cluster-C personality disorder groups. Behavioral data showed that BPD made more omission errors compared to NPC and CCP, whereas comparable commission errors were shown. Stimulus category had no effect on the number of omissions. Neuroimaging results showed higher activity in the FEF when inhibiting negative stimuli in BPD, while NPC showed less activity in the IPL when inhibiting negative stimuli. Furthermore, a marginally diminished response in the IPL was found during response inhibition of neutral stimuli in BPD compared to NPC. BPD patients showed a general responsivity across stimulus categories in the IPL, whereas effects in the FEF were specific for negative stimuli. In both clusters the comparison BPD versus CCP revealed no differences, indicating that activity in these clusters is not BPD-specific. Contrary to our expectations, these findings do not support the hypothesized impaired response inhibition in BPD.

The present study could not replicate the altered prefrontal activity (decreased vmPFC and IFC, increased lateral OFC and dIPFC) in BPD as reported in the two previous studies on BPD impulsivity (Jacob et al., 2013; Silbersweig et al., 2007). One explanation could be that we used a different go/no-go paradigm than previous studies. Silbersweig et al. (2007) used a linguistic go/no-go task, in which words were used, whereas we used pictures. Jacob et al. (2013) used a simple letter-based go/no-go task after an emotion induction, while we used a complex go/no-go task comprising emotional stimuli. Moreover, the current study had more statistical power than previous studies. Taken together, as fMRI studies examining the hypothesized impaired response inhibition in BPD comprise a diversity of task designs, more research is necessary to explain the underlying neurocircuits.

Investigation of diagnosis specificity indicates a linear relationship, with an intermediate response of the CCP, placed between NPC and BPD. This supports the idea that cluster-C patients exhibit common emotional and interpersonal problems (American Psychiatric Association, 2013) with BPD, and that the observed effects are more a dimensional rather than a categorical differentiation.

Results concerning stimulus category specificity showed that the IPL also showed a significant interaction involving positive and erotic stimuli, caused by an activity modulation across stimulus categories in NPC. This modulation by positive and erotic stimuli was absent in BPD. This result indicates, in contrast to the hypotheses that BPD is not affected by the stimulus category. However, the FEF did show higher activity during the presentation of the negative stimuli in BPD compared to the other stimuli categories. This effect was not present in the NPC. This might suggest that if top-down modulation does not work on the 
perceptional input channel at the level of the IPL, in BPD this must happen in the output channel of the FEF.

IPL activity during response inhibition is previously linked to attentional processes to go/nogo-stimuli (Simmonds et al., 2008; Swick et al., 2011). The left FEF and left IPL are advocated to be core regions in the left dorsal attention network, which is involved in topdown attentional control over brain areas of the sensory cortices and ventral attention network (Corbetta, Patel \& Shulman, 2008). Attention is a bottleneck in many information processing streams, and therefore might interfere with higher cognitive processes. Hence, we speculate that the findings of altered activity of the left dorsal attention network in BPD hints towards an impaired top-down attentional bias implementation and inappropriate top-down control over the ventral attention network. This might mean that BPD patients constantly shift their attention towards unimportant stimuli and/or that they have difficulties withdrawing their attention from emotional stimuli. Subsequently, continuous reorientation and/or slowed disengagement might interfere with cognitive resources required to disengage attention from sensory salient but behaviorally irrelevant stimuli. This is in line with behavioral studies, reporting on biased attention towards emotional stimuli in BPD (Arntz, Appels \& Sieswerda, 2000; von Ceumern-Lindenstjerna et al., 2010). Further this idea is supported by our behavioral data in which no group differences were shown for commissions, but more omissions were observed for BPD patients compared to NPC, indicating no deficiencies in response inhibition per se but pointing to attentional difficulties. However, this warrants further research using attention paradigms.

The strengths of the current study include an extension of stimulus categories, inclusion of the clinical control group and a well-powered sample size compared to previous studies (van Zutphen, Siep, Jacob, Goebel \& Arntz, 2015). There are also some limitations to be recognized. Firstly, only women were recruited, which limits the generalizability of our results to men. Secondly, patients were taking psychotropic medication for clinical reasons, which is a potentially confounding factor (Delaveau et al., 2011; Ma, 2015). However, excluding patients on medication would result in a non-representative and less severe clinical sample. As adding medication as a covariate removes variance associated with group differences, additional analyses within the BPD were performed. Interactions of medication within the BPD were not significant within any of the resulting brain areas, suggesting that medication did not influence the results. Thirdly, an unbalanced design was used in this study, in which blocks containing the same stimulus category for the go's and no-go's were missing and the neutral nogo-blocks were always presented in a block containing emotional go-trials. However, by using LMM we could control for the unbalanced design by adding the initial go's brain responses as covariates.

In conclusion, BPD patients showed altered responses in the IPL and FEF, when inhibiting emotional stimuli. BPD patients showed a general responsivity across stimulus categories 
in the IPL, whereas effects in the FEF were specific for negative stimuli. Linearity analyses implied a dimensional rather than a categorical differentiation, with responses of CCP inbetween NPC and BPD. In face of the current discussion on impulse control deficits in BPD (Sebastian et al., 2013a; van Eijk et al., 2015), the current results add further evidence in the view that interference control in BPD might be impaired in early processing stages, rather than in motor inhibitory control itself (Sebastian et al., 2013a; van Eijk et al., 2015). 


\section{References}

American Psychiatric Association. (2013). Diagnostic and Statistical Manual of Mental Disorder (5th ed.). Arlington, VA: American Psychiatric Association.

Arntz, A., Appels, C., \& Sieswerda, S. (2000). Hypervigilance in borderline disorder: a test with the emotional Stroop paradigm. Journal of Personality Disorders, 14, 366-373.

Arntz, A., \& Dreessen, L. (1995). BPD-Klachtenlijst 47 [BPD Checklist]. The Netherlands: Maastricht University.

Corbetta, M., Patel, G., \& Shulman, G. L. (2008). The reorienting system of the human brain: from environment to theory of mind. Neuron, 58, 306-324. doi: 10.1016/j.neuron.2008.04.017

Delaveau, P., Jabourian, M., Lemogne, C., Guionnet, S., Bergouignan, L., \& Fossati, P. (2011). Brain effects of antidepressants in major depression: a meta-analysis of emotional processing studies. Journal of Affective Disorders, 130, 66-74. doi: 10.1016/j.jad.2010.09.032

Derogatis, L. R. (1993). BSI Brief Symptom Inventory: Administration, Scoring, and Procedure Manual (4th ed.). Minneapolis, MN: National Computer Systems.

Domes, G., Winter, B., Schnell, K., Vohs, K., Fast, K., \& Herpertz, S. C. (2006). The influence of emotions on inhibitory functioning in borderline personality disorder. Psychological Medicine, 36, 1163-1172. doi: 10.1017/ S0033291706007756

Forman, S. D., Cohen, J. D., Fitzgerald, M., Eddy, W. F., Mintun, M. A., \& Noll, D. C. (1995). Improved assessment of significant activation in functional magnetic resonance imaging (fMRI): use of a cluster-size threshold. Magnetic Resonance in Medicine, 33, 636-647.

Goldstein, M., Brendel, G., Tuescher, O., Pan, H., Epstein, J., Beutel, M., .. Silbersweig, D. (2007). Neural substrates of the interaction of emotional stimulus processing and motor inhibitory control: an emotional linguistic go/ no-go fMRI study. Neurolmage, 36, 1026-1040. doi: 10.1016/j.neuroimage.2007.01.056

Jacob, G. A., Arntz, A., Domes, G., Reiss, N., \& Siep, N. (2011). Positive erotic picture stimuli for emotion research in heterosexual females. Psychiatry Research, 190, 348-351. doi: 10.1016/j.psychres.2011.05.044

Jacob, G. A., Gutz, L., Bader, K., Lieb, K., Tüscher, O., \& Stahl, C. (2010). Impulsivity in borderline personality disorder: impairment in self-report measures, but not behavioral inhibition. Psychopathology, 43, 180-188. doi: $10.1159 / 000304174$

Jacob, G. A., Zvonik, K., Kamphausen, S., Sebastian, A., Maier, S., Philipsen, A., . . Tüscher, O. (2013). Emotional modulation of motor response inhibition in women with borderline personality disorder: an fMRI study. Journal of Psychiatry \& Neuroscience, 38, 164-172. doi: 10.1503/jpn.120029

Koenigsberg, H. W., Fan, J., Ochsner, K. N., Liu, X., Guise, K. G., Pizzarello, S., .. Siever, L. J. (2009). Neural correlates of the use of psychological distancing to regulate responses to negative social cues: a study of patients with borderline personality disorder. Biological Psychiatry, 66, 854-863. doi: 10.1016/j.biopsych.2009.06.010

Lang, P. J., Bradley, M. M., \& Cuthbert, B. N. (1997). International Affective Picture System (IAPS): Instruction Manual and Affective Ratings. Gainesville, FL: Center of Research in Psychophysiology, University of Florida.

Leyton, M., Okazawa, H., Diksic, M., Paris, J., Rosa, P., Mzengeza, S., . . Benkelfat, C. (2001). Brain regional alpha-[11C] methyl-L-tryptophan trapping in impulsive subjects with borderline personality disorder. The American Journal of Psychiatry, 158, 775-782. doi: 10.1176/appi.ajp.158.5.775

Lieberman, M. D., \& Cunningham, W. A. (2009). Type I and Type II error concerns in fMRI research: re-balancing the scale. Social Cognitive and Affective Neuroscience, 4, 423-428. doi: 10.1093/scan/nsp052

Lobbestael, J., Arntz, A., Harkema-Schouten, P., \& Bernstein, D. (2009). Development and psychometric evaluation of a new assessment method for childhood maltreatment experiences: the interview for traumatic events in childhood (ITEC). Child Abuse \& Neglect, 33, 505-517. doi: 10.1016/j.chiabu.2009.03.002

Ma, Y. (2015). Neuropsychological mechanism underlying antidepressant effect: a systematic meta-analysis. Molecular Psychiatry, 20, 311-319. doi: 10.1038/mp.2014.24

McGlashan, T. H., Grilo, C. M., Skodol, A. E., Gunderson, J. G., Shea, M. T., Morey, L. C., . . Stout, R. L. (2000). The Collaborative Longitudinal Personality Disorders Study: baseline Axis I/II and II/I diagnostic co-occurrence. Acta Psychiatrica Scandinavica, 102, 256-264. doi: 10.1034/j.1600-0447.2000.102004256.x

Moeller, F. G., Barratt, E. S., Dougherty, D. M., Schmitz, J. M., \& Swann, A. C. (2001). Psychiatric aspects of impulsivity. The American Journal of Psychiatry, 158, 1783-1793. doi: 10.1176/appi.ajp.158.11.1783

Mortensen, J. A., Rasmussen, I. A., \& Håberg, A. (2010). Trait impulsivity in female patients with borderline personality disorder and matched controls. Acta Neuropsychiatrica, 22, 139-149. doi: 10.1111/j.1601-5215.2010.00468.x 
Ochsner, K. N., Silvers, J. A., \& Buhle, J. T. (2012). Functional imaging studies of emotion regulation: a synthetic review and evolving model of the cognitive control of emotion. Annals of the New York Academy of Sciences, 1251, E124. doi: 10.1111/j.1749-6632.2012.06751.x

Rentrop, M., Backenstrass, M., Jaentsch, B., Kaiser, S., Roth, A., Unger, J., . . Renneberg, B. (2008). Response inhibition in borderline personality disorder: performance in a Go/Nogo task. Psychopathology, 41, 50-57. doi: $10.1159 / 000110626$

Sebastian, A., Jacob, G., Lieb, K., \& Tüscher, O. (2013a). Impulsivity in borderline personality disorder: a matter of disturbed impulse control or a facet of emotional dysregulation? Current Psychiatry Reports, 15, 339. doi: 10.1007/s11920-012-0339-y

Sebastian, A., Pohl, M. F., Kloppel, S., Feige, B., Lange, T., Stahl, C., ... Tüscher, O. (2013b). Disentangling common and specific neural subprocesses of response inhibition. Neurolmage, 64, 601-615. doi: 10.1016/j. neuroimage.2012.09.020

Shafritz, K. M., Collins, S. H., \& Blumberg, H. P. (2006). The interaction of emotional and cognitive neural systems in emotionally guided response inhibition. Neurolmage, 31, 468-475. doi: 10.1016/j.neuroimage.2005.11.053

Silbersweig, D., Clarkin, J. F., Goldstein, M., Kernberg, O. F., Tuescher, O., Levy, K. N., . . Stern, E. (2007). Failure of frontolimbic inhibitory function in the context of negative emotion in borderline personality disorder. The American Journal of Psychiatry, 164, 1832-1841. doi: 10.1176/appi.ajp.2007.06010126

Simmonds, D. J., Pekar, J. J., \& Mostofsky, S. H. (2008). Meta-analysis of Go/No-go tasks demonstrating that fMRI activation associated with response inhibition is task-dependent. Neuropsychologia, 46, 224-232. doi: 10.1016/j. neuropsychologia.2007.07.015

Swick, D., Ashley, V., \& Turken, U. (2011). Are the neural correlates of stopping and not going identical? Quantitative meta-analysis of two response inhibition tasks. Neurolmage, 56, 1655-1665. doi: 10.1016/j. neuroimage.2011.02.070

van Eijk, J., Sebastian, A., Krause-Utz, A., Cackowski, S., Demirakca, T., Biedermann, S. V., . . Tüscher, O. (2015). Women with borderline personality disorder do not show altered BOLD responses during response inhibition. Psychiatry Research, 234, 3378-389. doi: 10.1016/j.pscychresns.2015.09.017

van Zutphen, L., Siep, N., Jacob, G. A., Domes, G., Sprenger, A., Willenburg, B., . . Arntz, A. (n.d.). Always on guard: Emotion regulation in borderline personality disorder compared to non-patients and cluster-C personality disorder patients. Submitted

van Zutphen, L., Siep, N., Jacob, G. A., Goebel, R., \& Arntz, A. (2015). Emotional sensitivity, emotion regulation and impulsivity in borderline personality disorder: A critical review of fMRI studies. Neuroscience and Biobehavioral Reviews, 51C, 64-76. doi: 10.1016/j.neubiorev.2015.01.001

Vollm, B., Richardson, P., Stirling, J., Elliott, R., Dolan, M., Chaudhry, I., .. . Deakin, B. (2004). Neurobiological substrates of antisocial and borderline personality disorder: preliminary results of a functional fMRI study. Criminal Behaviour and Mental Health, 14, 39-54. doi: 10.1002/cbm.559

von Ceumern-Lindenstjerna, I. A., Brunner, R., Parzer, P., Mundt, C., Fiedler, P., \& Resch, F. (2010). Attentional bias in later stages of emotional information processing in female adolescents with borderline personality disorder. Psychopathology, 43, 25-32. doi: 10.1159/000255960

Wetzelaer, P., Farrell, J., Evers, S., Jacob, G. A., Lee, C. W., Brand, O., . . Arntz, A. (2014). Design of an international multicentre RCT on group schema therapy for borderline personality disorder. BMC Psychiatry, 14, 319. doi: 10.1186/s12888-014-0319-3

Zanarini, M. C., Frankenburg, F. R., Dubo, E. D., Sickel, A. E., Trikha, A., Levin, A., \& Reynolds, V. (1998a). Axis I comorbidity of borderline personality disorder. The American Journal of Psychiatry, 155, 1733-1739.

Zanarini, M. C., Frankenburg, F. R., Dubo, E. D., Sickel, A. E., Trikha, A., Levin, A., \& Reynolds, V. (1998b). Axis II comorbidity of borderline personality disorder. Comprehensive Psychiatry, 39, 296-302. doi: 10.1016/S0010-440X(98)90038-4

Zanarini, M. C., Yong, L., Frankenburg, F. R., Hennen, J., Reich, D. B., Marino, M. F., \& Vujanovic, A. A. (2002). Severity of reported childhood sexual abuse and its relationship to severity of borderline psychopathology and psychosocial impairment among borderline inpatients. The Journal of Nervous and Mental Disease, 190, 381387. doi: 10.1097/00005053-200206000-00006

Zimmerman, M., \& Mattia, J. I. (1999). Axis I diagnostic comorbidity and borderline personality disorder. Comprehensive Psychiatry, 40, 245-252. doi: 10.1016/S0010-440X(99)90123-2 


\section{Supplementary Materials}

The text below is almost identical to van Zutphen et al. (submitted) due to two parallel studies performed by the same research groups.

\section{Methods}

\section{Participants}

Patients were recruited from mental health clinics at local sites, Virenze-Riagg Maastricht (The Netherlands), PsyQ Heerlen (The Netherlands), the BPD treatment unit of the Department of Psychiatry and Psychotherapy at the Medical Center Freiburg (Germany), the Department of Psychiatry and Psychotherapy at the University Hospital Lübeck (Germany) and the Institute for Behavior Therapy Training Hamburg (Germany). Borderline personality disorder (BPD) patients were recruited within the context of an international multicenter RCT on group schema therapy versus treatment-as-usual (Wetzelaer et al., 2014). Only females were chosen since gender might influence emotional processing (Whittle, Yucel, Yap \& Allen, 2011), and because in mental health care BPD is more often diagnosed in females. We excluded homosexual females, because we used heterosexual erotic stimuli. General exclusion criteria were lifetime psychotic or bipolar disorder type-l, attentiondeficit/hyperactivity disorder, dissociative identity disorder, serious and/or unstable medical illness, substance dependence needing clinical detoxification and fMRI exclusion criteria (i.e. claustrophobia, metal objects, cardiac arrhythmia, epilepsy, tattoos at neck/head and pregnancy).

BPD and cluster-C (CCP) patients underwent the Structural Clinical Interview (SCID) for Axis I (First, Spitzer, Gibbon \& Williams, 1994) and II (First, Spitzer, Gibbon, Williams \& Benjamin, 1997) assessed by trained interviewers and were diagnosed according to the DSM-IV criteria. Measurements were collected before start of patients' therapy, unless impossible due to scheduling problems fMRI-measurements had to be finished within three months from the start of therapy. BPD patients that scored full or sub-threshold on narcissistic and antisocial PD were excluded for reasons related to the clinical trial in which this study sample participated (Wetzelaer et al., 2014). Moreover, BPD patients were further screened by means of BPD Severity Index (Arntz et al., 2003; Giesen-Bloo, Wachters, Schouten \& Arntz, 2010; Kroger et al., 2013), for inclusion this score was > 20 (31.36 \pm 6.86 ). CCP were not allowed to score full or sub-threshold Cluster-B PD, or $>2$ BPD criteria. Nonpatients (NPC) did not meet current diagnostic criteria for any Axis I or II disorder. They were screened with the SCID I and II screeners (First et al., 1994; First et al., 1997), positive items on the screeners were checked with SCID interviews.

Three BPD patients, one NPC and one CCP were excluded because of invalid or incomplete data, two NPC and one CCP because of too much motion (if head motion in 2 
or more runs was $>4 \mathrm{~mm}$ ) during scanning, and one NPC and one CCP because of incorrect responses on the behavioral data, two NPC because of scores above .70 on the BSI and three CCP because of scores above 100 on the BPD checklist. Finally, three BPD and two CCP were excluded because they had an estimated IQ outside the range of 75-120. Additionally, as there was a disproportionate number of NPC with IQ higher than the highest IQ in the BPD group, we excluded one NPC with IQ scores above the 95-percentile to guarantee a better match between NPC and BPD patients.

\section{Measures}

\section{Brief Symptom Inventory (BSI)}

The BSI is a brief psychological self-report inventory of general symptoms of psychopathology during the past week (Derogatis, 1993). It is a short alternative for the Symptom Checklist-90-R from which it was developed. It contains 53 items divided over nine dimensions: somatization, obsession-compulsion, interpersonal sensitivity, depressive mood, anxiety, hostility, phobic anxiety, paranoid ideation and psychoticism. Answers are scored on a 5-point Likert scale, ranging from 0 (not at all) to 4 (extremely). Scores of the dimensions are calculated by summing the values for the items divided by the number of items within the subscales. The total score measures the level of symptomatology, which is the sum of the nine dimensions plus the four additional items divided by total number of items. The internal consistency showed a Cronbach's a of .96 for the total instrument and ranged between .71 and .85 for its subscales (De Beurs \& Zitman, 2006; Derogatis, 1993). To distinguish patients from non-patients a cutoff score of 70 is suggested (De Beurs, 2004).

\section{BPD Checklist}

The BPD Checklist is a self-report questionnaire used to assess the burden of BPD symptoms as experienced during the last month (Arntz \& Dreessen, 1995). It consists of 47 items based on the nine dimensions of BPD in DSM-IV. Items must be rated on 5-point Likert scale, ranging from 1 (not at all) to 5 (extremely). Next to the total sumscore, also the scores for the nine subscales can be calculated. Scores above 100 signify BPD-pathology. When control patients showed an elevated score, an extra check with the SCID II BPD section was done.

\section{Interview for Traumatic Events in Childhood (ITEC)}

The ITEC is a retrospective, semi-structured interview to measure childhood maltreatment prior the age of 18 , including sexual (12 items), physical (13 items) and emotional abuse (9 items), and emotional ( 6 items) and physical neglect (15 items) (Lobbestael, Arntz, HarkemaSchouten \& Bernstein, 2009). For each item the participant experienced maltreatment, follow-up questions are used to gather more detailed information about the perpetrator(s), 
age of onset, frequency, duration of the trauma and the impact on the victim in the past and in the present. This information was used to calculate a severity score between 0 and 1 , such that the score increased with the severity of the event itself, the closeness of the perpetrator, the younger age of onset, the longer duration of the event, and the higher impact on the victim. For each subscales the severity scores for the events are summed, the higher this score, the more severe the maltreatment. Internal consistencies of these scales were moderate to excellent, with Cronbach's a varying between .58 and .89 with a mean of .79 (Lobbestael et al., 2009). In addition to the victimization scales, similar scales were created for witnessing the various forms of maltreatment. In current study only the victim scales are used. One average the administration time is about 30 minutes but can take up to one hour in case of multiple maltreatments.

\section{Dissociation and Anxiety}

Present state dissociative experiences were assessed using four items of the DissociationTension-Scale (Stiglmayr, Shapiro, Stieglitz, Limberger \& Bohus, 2001), containing derealization and changes in perception of one's body, hearing and pain. Additionally two items concerning the level of anxiety and the level of nervousness were added. Responses were indicated on a visual analogue scale, ranging from 0 (not at all) to 10 (extremely). The dissociation score was the averaged across the four dissociation items. Internal consistencies of the dissociation score proved to be good in current sample, with a Cronbach's $a$ of .86 concerning dissociation before scanning and Cronbach's $a=.87$ concerning dissociation after scanning.

\section{Self-Assessment Manikin Scale}

The pictures shown during the scanning session were qualitatively assessed for valence and arousal using the Self-Assessment Manikin Scale. The Self-Assessment Manikin Scale consists of a series of human-like figures to measures the affective reaction of a person to stimuli (Bradley \& Lang, 1994). Intensity of valence and arousal were both rated on a 9-point scale, with for valence 1 being extremely unpleasant and 9 being extremely pleasant, and for arousal 1 being most calm and 9 being most aroused.

\section{Wechsler Adult Intelligence Scale}

IQ was estimated by means of four subtests of the Wechsler Adult Intelligence Scale, including two verbal (i.e. Vocabulary and Similarities) and two nonverbal tests (i.e. Block design and Matrix reasoning). Together these subtests correlate strongly with general intellectual ability (Schrimsher, O'Bryant, O'Jile \& Sutker, 2008). IQ was estimated based on the optimized regression equation: $39.05+(1.54 *$ comprehension score $)+(1.64 *$ matrix 
reasoning score $)+(1.48 *$ similarities score $)+(.98 *$ picture arrangement score). If the WAIS score was not available (BPD $n=12$ and NPC $n=1$ ) an estimation of the IQ was made based on the education level, using the regression equation from our present sample per group; IQ BPD $=85.932+(3.360 *$ ISCED code) and IQ NPC $=85.996+(3.659 *$ ISCED code). These regression equations explained respectively $22 \%$ and $31 \%$ of the variance in IQ. Level of education of both the Dutch and German educational systems were transformed into the International Standard Classification of Education (ISCED).

\section{Borderline Personality Disorder Severity Index (BPDSI)}

The BPDSI is a semi-structured clinical interview assessing frequency and severity of BPD manifestations (Arntz et al., 2003; Giesen-Bloo et al., 2010; Kroger et al., 2013). The 70-items reflect the nine BPD criteria described in the DSM-IV. For each item the frequency of the last three months is rated on an 11-point scale, ranging from 0 (never) to 10 (daily). The scores on the subscales provide information on the severity of each of the DSM-IV dimensions, derived by averaging the items scores. The total score is the sum of the nine dimensions scores, ranging from 0 to 90, with an internal consistency of Cronbach's $a=.93$ and subscales ranged between .41 and .83 (Arntz et al., 2003). A total score of 20 distinguishes BPD from other personality disorders (Giesen-Bloo et al., 2010).

\section{Procedure}

Prior to scanning all participants were trained on a practice task outside the scanner. This task was similar to the experimental task inside the scanner. After finishing the practice task the participant entered the scanner and the scanning session of 75 minutes was completed. Presentation of the stimuli and recordings of behavioral responses were controlled by Presentation (Neurobehavioral Systems Inc., Albany, CA, USA). The visual stimuli were projected via $\mathrm{PC}$ and beamer onto a screen that was viewed through a mirror on the headcoil or via a goggle-system. As part of the scanning session participants also underwent two resting state scans (data reported separately). At the end of the session the participant completed an 'exit'-questionnaire, assessing information about their general experience with the fMRI. In addition, before and after scanning the anxiety and dissociation state was assessed (Stiglmayr et al., 2001). Finally, the participants had to rate their subjective reaction of each picture they had seen during the session.

\section{fMRI acquisition}

Functional MRI was performed on 3T scanners at all three sites, at Maastricht on a Siemens Magnetom Allegra head-only scanner equipped with a birdcage headcoil (Siemens Medical Systems, Erlangen, Germany), at Freiburg on a Siemens tim-Trio Magnetom whole 
body scanner (Siemens Medical Systems, Erlangen, Germany) equipped with an 8-channel headcoil, and at Lübeck on a Philips Achiva whole body scanner equipped with an 8-channel headcoil (Philips Healthcare, Best, The Netherlands). The BPD patients from Heerlen were scanned in Maastricht and from Hamburg were scanned in Lübeck. In Maastricht 11 BPD, 10 NPC and 11 CCP were scanned, Freiburg scanned 13 BPD, 11 NPC and 6 CCP, finally 29 BPD, 13 NPC and 4 CCP were scanned in Lubeck.

Participants were scanned in head first supine position. Head movements were minimalized using foam paddings. Additionally, the participant was instructed to avoid moving as much as possible during scanning. T2*-weighted images were acquired via echo planar imaging (EPI), using the following imaging parameters: TR $=2000 \mathrm{~ms}$, TE $=27 \mathrm{~ms}$, flip angle $=90^{\circ}$, FoV $=192 \times 192 \mathrm{~mm}$, voxel size $=3 \times 3 \times 3 \mathrm{~mm}$, and matrix $=64 \times 64$. Images were recorder in four runs of 280 images in Maastricht, 276 images in Freiburg and 256 images Lübeck. One volume in Maastricht consisted of 32, and in Freiburg and Lübeck of 34 , interleaved measured axial slices. A slice tilt correction of $-30^{\circ}$ was used to optimize the susceptibility and minimize the distortion artifacts within the amygdala the $T 2^{*}$-weighted images (Morawetz et al., 2008) in Maastricht and Freiburg. A whole-brain anatomical scan in sagittal plane was acquired, using a high resolution T1-weigthed sequence (TR $=2250 \mathrm{~ms}$, $\mathrm{TE}=2.6 \mathrm{~ms}$, flip angle $=9^{\circ}, \mathrm{FoV}=256 \times 256 \mathrm{~mm}$, voxel size $\left.1 \times 1 \times 1 \mathrm{~mm}\right)$. In total, 192 images were obtained in Maastricht, 160 in Freiburg and 170 in Lübeck.

\section{fMRI preprocessing}

All preprocessing and statistical analyses were performed with BrainVoyager 2.6 (Brain Innovation, Maastricht, The Netherlands). The first two images of each run were discarded because of saturation effects. Preprocessing contained slice time correction with sinc interpolation, 3D motion correction for three translation and three rotation parameters with trilinear interpolation for detection and sinc interpolation for motion correction and removal of low-frequency drifts was performed by high-pass temporal filtering of 2 sines/cosines per run (Goebel, Espositio \& Formasino, 2006). To improve data quality all anatomical scans were peeled from the skull and corrected for intensity inhomogeneities. Participants underwent another session concerning a different task (data presented elsewhere, van Zutphen et al., submitted), in which also an anatomical scan was conducted. To obtain a high resolution and high contrast anatomical scan, both anatomical scans were averaged when possible. After preprocessing the functional data were coregistered with the anatomical data per run, and for each run a volume-time-course was created. Each volume-time-course was spatially smoothed with a $6 \mathrm{~mm}$ full-width-at-half-maximum isotropic Gaussian kernel. Spatial normalization was performed using standard Talairach transformation procedures (Talairach and Tournoux, 1988). 


\section{Supplementary Results}

\section{Analyses of BPD patients compared to both control groups of inhibition}

The whole brain random effects ANOVA F-map: inhibition (no-go vs. go) x group (BPD vs. NPC) resulted in two clusters, which were anatomically identified at the left superior temporal gyrus (peak Tal: $x=32, y=-52, z=15 ; B A 22 ; 381 \mathrm{~mm}^{3} ; F_{1,85}=14.51, p<.001$ ) and left hippocampus (peak Tal: $x=-34, y=-20, z=-9 ; 521 \mathrm{~mm}^{3} ; F_{1,85}=9.88, p=.002$ ). In both areas simple effects showed less activity in the NPC for no-go versus go. Furthermore, in the superior temporal gyrus BPD patients showed more activity for no-go versus go. No differences were found for the comparison of BPD and CCP. 


\section{References}

Arntz, A., \& Dreessen, L. (1995). BPD-Klachtenlijst 47 [BPD Checklist]. The Netherlands: Maastricht University.

Arntz, A., van den Hoorn, M., Cornelis, J., Verheul, R., van den Bosch, W. M., \& de Bie, A. J. (2003). Reliability and validity of the borderline personality disorder severity index. Journal of Personality Disorders, 17, 45-59. doi: 10.1521/ pedi.17.1.45.24053

Bradley, M. M., \& Lang, P. J. (1994). Measuring emotion: the Self-Assessment Manikin and the Semantic Differential. Journal of Behavior Therapy and Experimental Psychiatry, 25, 49-59. doi: 10.1016/0005-7916(94)90063-9

De Beurs, E. (2004). Handleiding bij de Brief Symptom Inventory (BSI). Leiden: Pits Publishers.

De Beurs, E., \& Zitman, F. G. (2006). De Brief Symptom Inventory (BSI): De betrouwbaarheid en validiteit van een handzaam alternatief voor de SCL-90 [The Brief Symptom Inventory (BSI): The reliability and validity of a brief alternative of the SCL-90]. Maandblad Geestelijke Volksgezondheid, 61, 120-141.

Derogatis, L. R. (1993). BSI Brief Symptom Inventory: Administration, Scoring, and Procedure Manual (4th ed.) Minneapolis, MN: National Computer Systems.

First, M. B., Spitzer, R. L., Gibbon, M., \& Williams, J. B. W. (1994). Structured clinical interview for DSM-IV axis I disorders (SCID-I). New York: Biometric Research Department.

First, M. B., Spitzer, R. L., Gibbon, M., Williams, J. B. W., \& Benjamin, L. (1997). Structured clinical interview for DSM-IV axis II personality disorders (SCID-II). New York: Biometric Research Department.

Giesen-Bloo, J. H., Wachters, L. M., Schouten, E., \& Arntz, A. (2010). The Borderline Personality Disorder Severity IndexIV: psychometric evaluation and dimensional structure. Personality and Individual Differences, 49, 136-141. doi: DOI 10.1016/j.paid.2010.03.023

Goebel, R., Esposito, F., \& Formisano, E. (2006). Analysis of functional image analysis contest (FIAC) data with brainvoyager QX: From single-subject to cortically aligned group general linear model analysis and selforganizing group independent component analysis. Human Brain Mapping, 27, 392-401. doi: 10.1002/ hbm.20249

Kroger, C., Vonau, M., Kliem, S., Roepke, S., Kosfelder, J., \& Arntz, A. (2013). Psychometric properties of the German version of the borderline personality disorder severity index--version IV. Psychopathology, 46, 396-403. doi: $10.1159 / 000345404$

Lobbestael, J., Arntz, A., Harkema-Schouten, P., \& Bernstein, D. (2009). Development and psychometric evaluation of a new assessment method for childhood maltreatment experiences: the interview for traumatic events in childhood (ITEC). Child Abuse \& Neglect, 33, 505-517. doi: 10.1016/j.chiabu.2009.03.002

Morawetz, C., Holz, P., Lange, C., Baudewig, J., Weniger, G., Irle, E., \& Dechent, P. (2008). Improved functional mapping of the human amygdala using a standard functional magnetic resonance imaging sequence with simple modifications. Magnetic Resonance Imaging, 26, 45-53. doi: 10.1016/j.mri.2007.04.014

Schrimsher, G. W., O'Bryant, S. E., O'Jile, J. R., \& Sutker, P. B. (2008). Comparison of tetradic WAIS-III short forms in predicting full scale IQ scores in neuropsychiatric clinic settings. Journal of Psychopathology and Behavioral Assessment, 30, 235-240. doi: DOI 10.1007/s10862-007-9066-9

Stiglmayr, C. E., Shapiro, D. A., Stieglitz, R. D., Limberger, M. F., \& Bohus, M. (2001). Experience of aversive tension and dissociation in female patients with borderline personality disorder -- a controlled study. Journal of Psychiatric Research, 35, 111-118. doi: 10.1016/S0022-3956(01)00012-7

van Zutphen, L., Siep, N., Jacob, G. A., Domes, G., Sprenger, A., Willenburg, B., . . Arntz, A. (n.d.). Always on guard: Emotion regulation in borderline personality disorder compared to non-patients and cluster-C personality disorder patients. Submitted

Wetzelaer, P., Farrell, J., Evers, S., Jacob, G. A., Lee, C. W., Brand, O., . . Arntz, A. (2014). Design of an international multicentre RCT on group schema therapy for borderline personality disorder. BMC Psychiatry, 14, 319. doi: 10.1186/s12888-014-0319-3

Whittle, S., Yucel, M., Yap, M. B., \& Allen, N. B. (2011). Sex differences in the neural correlates of emotion: evidence from neuroimaging. Biological Psychology, 87, 319-333. doi: 10.1016/j.biopsycho.2011.05.003 



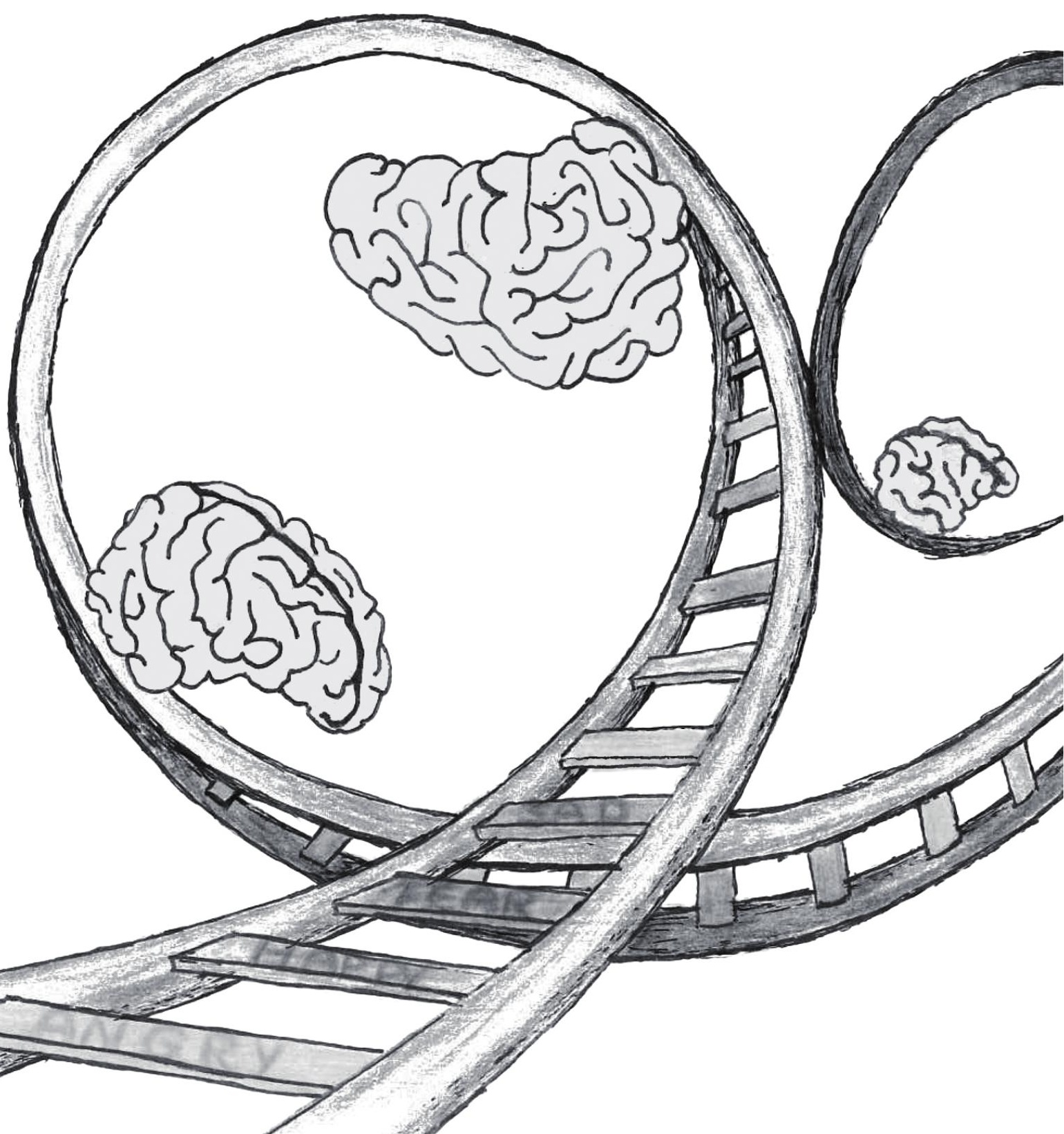




\section{Deficient amygdala-prefrontal intrinsic connectivity after effortful emotion regulation in borderline personality disorder}

This chapter has been accepted as:

Baczkowski, B.M.*, van Zutphen, L.*, Siep, N., Jacob, G.A., Domes, G., Maier, S. Sprenger, A., Senft, A., Willenborg, B., Tüscher, O., Arntz, A. $\&$ van de Ven, V. (2016). Deficient amygdalaprefrontal intrinsic connectivity after effortful emotion regulation in borderline personality disorder. European Archives of Psychiatry and Clinical Neuroscience. doi: 10.1007/s00406-0160760-z

* Authors contributed equally to this work 


\begin{abstract}

\section{Background}

Emotion instability in borderline personality disorder (BPD) has been associated with an impaired fronto-limbic inhibitory network. However, functional connectivity (FC) underlying altered emotion regulation in BPD has yet to be established. Here, we used resting-state fMRI to investigate enduring effects of effortful emotion regulation on the amygdala intrinsic FC in BPD.
\end{abstract}

\title{
Methods
}

In this multicenter study, resting-state $\mathrm{fMRI}$ was acquired before and after an emotion regulation task in 48 BPD patients and 39 non-patient comparison individuals. The bilateral amygdalae were used as a seed in the whole-brain FC analysis and two-way mixed ANOVA to test whether BPD patients exhibited weaker post-task increase in the amygdala intrinsic FC with the prefrontal cortex (PFC), compared to non-patients. Subsequently, we explored whether the results are common for personality disorders characterized by emotional problems, using additional data of 21 cluster-C personality disorder patients.

\section{Results}

In contrast to non-patients, BPD patients failed to show increased post-task amygdala resting-state FC with the medial, dorsolateral, ventrolateral PFC, and superior temporal gyrus, but surprisingly exhibited decreased FC with the posterior cingulate cortex and increased FC with the superior parietal lobule.

\section{Conclusions}

In BPD patients, the emotion regulation task failed to increase resting-state amygdala FC with brain regions essential for effortful emotion regulation, which suggests: (a) altered cognitive control typically used to indirectly alleviate distress by reinterpreting the meaning of emotional stimuli; (b) impaired direct regulation of emotional responses, which might be common for personality disorders; (c) avoidance of self-related appraisals induced by social emotional stimuli.

Keywords: BPD, emotion regulation, amygdala, functional connectivity, resting-state fMRI 


\section{Introduction}

Borderline personality disorder (BPD) is a severe psychiatric disorder with the prevalence in the general population estimated at approximately 1-3\% (Lenzenweger, Lane, Loranger \& Kessler, 2007; Trull, Jahng, Tomko, Wood \& Sher, 2010). Patients with BPD are characterized by a pervasive pattern of instability in self-image, interpersonal relationships, affect, and impulsive behavior (DSM-V, American Psychiatric Association, 2013). Leading conceptualizations posit that BPD is best understood as a disorder of emotion regulation (Carpenter \& Trull, 2013; Linehan, 1993; Sebastian, Jacob, Lieb \& Tüscher, 2013).

Functional neuroimaging studies highlight that clinically well-observed BPD features of emotion dysregulation - emotional hypersensitivity and intense emotional reactions - are due to increased limbic and diminished prefrontal activity (for meta-analysis and reviews: Krause-Utz, Winter, Niedtfeld \& Schmahl, 2014c; Schulze, Schmahl \& Niedtfeld, 2015; van Zutphen, Siep, Jacob, Goebel \& Arntz, 2015), which suggests an impaired fronto-limbic inhibitory network. Altered subcortical-cortical functional connectivity (FC) of BPD patients has been identified with FMRI during experimental induction of negative emotions (Cullen et al., 2011; Kamphausen et al., 2013; Niedtfeld et al., 2012) and in task-free resting-state (e.g. Krause-Utz et al., 2014b). Compared to non-patients (NPC), BPD patients exhibit increased amygdala FC with the subgenual anterior cingulate cortex (ACC) when viewing fearful faces (Cullen et al., 2011) and with the ventromedial prefrontal cortex (VmPFC) when exposed to threat (Kamphausen et al., 2013). In BPD, induction of pain together with emotionally arousing pictures is associated with enhanced negative FC of the amygdala with the medial and dorsolateral PFC (Niedtfeld et al., 2012). Furthermore, BPD patients exhibit stronger FC of both the amygdala and dorsal ACC with the dorsomedial prefrontal cortex (dmPFC) during emotional distraction (Krause-Utz et al., 2014a). Resting-state fMRI studies showed that emotional hypersensitivity of BPD patients is associated with hyperconnectivity within the salience network (Menon, 2015), i.e. between the amygdala and bilateral insula together with dorsal ACC (Das, Calhoun \& Malhi, 2014; Doll et al., 2013; Krause-Utz et al., 2014b; Salvador et al., 2014; Wolf et al., 2011), while their impaired control over emotional reactions is associated mostly with diminished intrinsic connectivity between the central executive fronto-parietal regions and salience network (Das et al., 2014; Doll et al., 2013). Both Doll and colleagues (2013) and Wolf and colleagues (2011) reported aberrant FC between the regions of the central executive fronto-parietal network at rest. Taken together, BPD patients show altered FC within the amygdala-PFC network when confronted with negative emotions, which is assumed to contribute to their affective instability and in the long-term shapes the organization of their resting-state networks.

However, brain FC associated with emotion regulation in BPD patients has yet to be established. Despite long-term stability of resting-state networks, resting-state functional connectivity ( $\mathrm{rsFC}$ ) exhibits substantial variations at the timescales of minutes (for review, 
Hutchison et al., 2013) and has been successfully used to investigate post-task changes in FC induced by cortical activation during behavioral manipulations (e.g. Grigg \& Grady, 2010; Lewis, Baldassarre, Committeri, Romani \& Corbetta, 2009), and stress exposure (Cremers et al., 2015; Veer et al., 2011) or memory processes (Gordon, Breeden, Bean \& Vaidya, 2014; Tambini, Ketz \& Davachi, 2010). Hence, resting-state fMRI can be used to investigate whether effortful emotion regulation induces enduring aberrant patterns of FC in BPD patients.

In the present international multicenter study, resting-state fMRI data were acquired before and after an emotion regulation task in 48 BPD patients and 39 NPC. We used an amygdala seed-based approach, since the amygdala is involved in emotion generation and its activity can be modulated by the prefrontal regions during effortful emotion regulation (Buhle et al., 2014; Kohn et al., 2014; Ochsner, Silvers \& Buhle, 2012). The increased coupling of the amygdala with the prefrontal regions is typically present during active regulation and consequently may alleviate emotional distress (Banks, Eddy, Angstadt, Nathan \& Phan, 2007; Urry et al., 2006). We hypothesized that in NPC effortful emotion regulation would lead to increased post-task amygdala rsFC with these prefrontal regions, whereas BPD patients would exhibit weaker increases. Subsequently, we explored whether the results are specific for BPD or common for personality disorders, including 21 cluster- $C$ personality disorder (CCP) patients. BPD patients are often additionally diagnosed with one of the CCPs (Zanarini et al., 1998), and emotional problems are common for both disorders, which, however, might be associated with different neurocognitive mechanisms (Koenigsberg et al., 2014).

\section{Materials and Methods}

\section{Participants}

Sixty-two BPD patients, 48 NPC, and 31 CCP patients were recruited from two sites in the Netherlands (Maastricht, Heerlen) and three sites in Germany (Freiburg, Lübeck, Hamburg). BPD and CCP patients were recruited from mental health clinics at local sites. NPC were recruited among the general population at each site. Participants had to be hetero- or bisexual females, aged 18-65, and sufficient understanding of the language of the local sites. Participants who did not fulfill the scanning or clinical criteria were excluded, and the final sample comprised 48 BPD patients, 39 NPC, and 21 CCP patients. Detailed description of additional measures including: BPD Severity Index (BPDSI; Arntz et al., 2003; GiesenBloo, Wachters, Schouten \& Arntz, 2010; Kroger et al., 2013), Brief Symptom Inventory (BSI; Derogatis, 1993), BPD checklist (Arntz \& Dreessen, 1995) and Interview for Trauma Events in Childhood (ITEC; Lobbestael, Arntz, Harkema-Schouten \& Bernstein, 2009), participant recruitment and exclusion procedure, is provided in the supplementary material.

Demographics and clinical measures of the sample are presented in Table 1. The groups did not significantly differ for age, handedness preference, and IQ. After complete 
description of the study, all participants provided written informed consent and received financial remuneration for their participation. The study was approved by the local medical ethical committees (Wetzelaer et al., 2014).

Table 1. Descriptive statistics of the three groups: borderline personality disorder (BPD), non-patient comparison individuals (NPC), and cluster-C personality disorder (CCP).

\begin{tabular}{|c|c|c|c|c|c|c|c|c|}
\hline & \multicolumn{2}{|c|}{$\begin{array}{c}\text { BPD } \\
(n=48) \\
\end{array}$} & \multicolumn{2}{|c|}{$\begin{array}{c}\text { NPC } \\
(n=39) \\
\end{array}$} & \multicolumn{2}{|c|}{$\begin{array}{c}\text { CCP } \\
(n=21) \\
\end{array}$} & \multicolumn{2}{|c|}{ Test Statistics } \\
\hline & Mean & SD & Mean & SD & Mean & SD & $F$ & $p$ \\
\hline Age (years) & 30.79 & 9.21 & 28.67 & 10.70 & 31.48 & 11.80 & .67 & .512 \\
\hline Estimated $I^{a}$ & 96.88 & 10.08 & 100.73 & 11.38 & 98.45 & 9.26 & 1.45 & $.239^{b}$ \\
\hline Brief Symptom Inventory & 1.68 & .55 & .13 & .13 & 1.11 & .43 & 142.19 & $<.001^{\mathrm{c}}$ \\
\hline BPD checklist & 118.44 & 25.58 & 50.68 & 5.21 & 74.80 & 17.31 & 135.21 & $<.001^{c}$ \\
\hline Interview Traumatic Events Childhood & & & & & & & 8.85 & $<.001^{\mathrm{d}}$ \\
\hline Sexual abuse & 9.44 & 8.85 & .13 & .42 & 2.26 & 5.43 & 21.21 & $<.001$ \\
\hline Physical abuse & 17.15 & 12.40 & 1.69 & 3.61 & 7.00 & 10.95 & 23.68 & $<.001$ \\
\hline Emotional abuse & 20.35 & 8.93 & 2.47 & 3.32 & 13.06 & 8.49 & 54.52 & $<.001$ \\
\hline Emotional neglect & 11.28 & 6.99 & .82 & 2.07 & 6.14 & 6.55 & 31.66 & $<.001$ \\
\hline Physical neglect & 10.68 & 9.42 & .88 & 2.82 & 4.35 & 6.90 & 17.67 & $<.001$ \\
\hline Dissociatione & & & & & & & 5.00 & $<.01^{f}$ \\
\hline prior scanning & 19.81 & 19.41 & 5.18 & 6.60 & 6.37 & 6.98 & 13.55 & $<.001^{9}$ \\
\hline \multirow[t]{2}{*}{ post scanning } & 31.87 & 26.85 & 6.40 & 7.81 & 15.36 & 20.60 & 16.02 & $<.001^{9}$ \\
\hline & $\%$ & n & $\%$ & n & $\%$ & $n$ & $x^{2}$ & $p$ \\
\hline Education level ${ }^{h}$ & & & & & & & 7.70 & $.02^{i}$ \\
\hline Level 1 & 22.9 & 11 & 17.9 & 7 & 14.3 & 3 & & \\
\hline Level 2 & 14.6 & 7 & 5.1 & 2 & 19.0 & 4 & & \\
\hline Level 3 & 27.1 & 13 & 10.3 & 4 & 28.6 & 6 & & \\
\hline Level 4 & 4.2 & 2 & 5.1 & 2 & 14.3 & 3 & & \\
\hline Level 5 & 25.0 & 12 & 43.6 & 17 & 14.3 & 3 & & \\
\hline Level 6 & 6.3 & 3 & 17.9 & 7 & 9.5 & 2 & & \\
\hline Handedness & & & & & & & 6.00 & $.20^{\mathrm{j}}$ \\
\hline Left & 8 & 4 & 5 & 2 & - & - & & \\
\hline Right & 86 & 41 & 95 & 37 & 100 & 21 & & \\
\hline Mixed & 6 & 3 & - & - & - & - & & \\
\hline \multicolumn{9}{|l|}{ Axis I disorders } \\
\hline Major depressive disorder & 87.5 & 42 & & & 61.9 & 13 & 5.92 & .02 \\
\hline Dysthymic & 8.3 & 4 & & & 4.8 & 1 & .28 & .60 \\
\hline Bipolar type II & 2.1 & 1 & & & - & - & .44 & .51 \\
\hline Generalized anxiety disorder & 4.2 & 2 & & & 4.8 & 1 & .12 & .91 \\
\hline Panic disorder with agoraphobia & 12.5 & 6 & & & - & - & 2.88 & .09 \\
\hline Panic disorder & 12.5 & 6 & & & 14.3 & 3 & .41 & .84 \\
\hline Agoraphobia & 8.3 & 4 & & & - & - & 1.86 & .17 \\
\hline Specific phobia & 18.8 & 9 & & & 4.8 & 1 & 2.31 & .13 \\
\hline
\end{tabular}


Table 1. Descriptive statistics of the three groups: borderline personality disorder (BPD), non-patient comparison individuals (NPC), and cluster-C personality disorder (CCP). (Continued)

\begin{tabular}{|c|c|c|c|c|c|c|c|c|}
\hline & \multicolumn{2}{|c|}{$\begin{array}{c}\text { BPD } \\
(n=48)\end{array}$} & \multicolumn{2}{|c|}{$\begin{array}{c}\text { NPC } \\
(n=39)\end{array}$} & \multicolumn{2}{|c|}{$\begin{array}{c}\text { CCP } \\
(n=21)\end{array}$} & \multicolumn{2}{|c|}{ Test Statistics } \\
\hline & $\%$ & $n$ & $\%$ & $\mathrm{n}$ & $\%$ & $\mathrm{n}$ & $x^{2}$ & $p$ \\
\hline Social phobia & 31.2 & 15 & & & 23.8 & 5 & .39 & .53 \\
\hline Obsessive compulsive disorder & 14.6 & 7 & & & 9.5 & 2 & .33 & .57 \\
\hline Posttraumatic stress disorder & 35.4 & 17 & & & 14.3 & 3 & 3.17 & .08 \\
\hline Somatoform disorder & 10.4 & 5 & & & 19.0 & 4 & .96 & .33 \\
\hline Eating disorders & 35.4 & 17 & & & 33.3 & 7 & .03 & .87 \\
\hline Substance abuse & 43.8 & 21 & & & 4.8 & 1 & 10.23 & .001 \\
\hline Intermitted explosive disorder & 2.1 & 1 & & & - & - & .44 & .51 \\
\hline \multicolumn{9}{|l|}{ Axis II disorders } \\
\hline Avoidant PD & 43.8 & 21 & & & 71.4 & 15 & 4.49 & .03 \\
\hline Dependent PD & 14.6 & 7 & & & 9.5 & 2 & .33 & .57 \\
\hline Obsessive compulsive PD & 20.8 & 10 & & & 33.3 & 7 & 1.23 & .27 \\
\hline Passive aggressive PD & 6.2 & 3 & & & - & - & 1.37 & .24 \\
\hline Depressive PD & 25.0 & 12 & & & 9.5 & 2 & 2.16 & .14 \\
\hline Paranoid PD & 29.2 & 14 & & & - & - & 7.68 & $<.01$ \\
\hline Schizotypal PD & 2.1 & 1 & & & - & - & .44 & .51 \\
\hline Schizoid PD & 2.1 & 1 & & & - & - & .44 & .51 \\
\hline \multicolumn{9}{|l|}{ Medication } \\
\hline Antidepressants & 64.6 & 31 & & & 36.4 & 8 & 4.17 & .04 \\
\hline Antipsychotics & 10.4 & 5 & & & - & - & 2.36 & .13 \\
\hline Hypnotics & 4.2 & 2 & & & - & - & .90 & .34 \\
\hline Mood Stabilizers & 2.1 & 1 & & & - & - & .44 & .51 \\
\hline
\end{tabular}

a Assessed with four subtasks of the WAIS (i.e. vocabulary, similarities, block design, and matrix reasoning).

${ }^{b}$ Data of one NPC was not available.

cAll three groups significantly differed from each other $(p<.001)$. Data of two NPC and one CCP patient were not available.

${ }^{d}$ MANOVA and ANOVAs showed significant group differences over the childhood traumatic events. BPD patients reported more traumatic events than either CCP patients or NPC with respect to sexual abuse, physical abuse, emotional abuse, emotional neglect, and physical neglect (all $p s<.01$ ). CCP patients reported more traumatic events than NPC with respect to emotional abuse and emotional neglect (all $p s<.01$ ). Data from seven NPC and one CCP patient were not available.

e Measured with the four somatic dissociation items of the Dissociation-Tension-Scale (i.e. derealization and changes in perception of one's body, hearing, and pain).

${ }^{\mathrm{f}}$ Two-way mixed-design ANOVA showed a significant group $\times$ time interaction. In BPD patients, the increase in reported dissociative states was significantly larger than in NPC $(p=.001)$. Data from five BPD patients, two NPC, and one CCP patient were not available.

9 ANOVA showed a significant group effect over dissociation. BPD patients showed increased level of dissociation as compared to NPC $(p<.001)$ as well as to CCP group $(p<.001)$.

${ }^{\mathrm{h}}$ Level of education of both the Dutch and German educational systems was translated into the International Standard Classification of Education (ISCED), and in the current study, six levels of education were divided ranging from lower secondary school to Master's degree.

'Value is based on the Kruskal-Wallis test. Data of one NPC was not available.

JValue is based on the Chi-square goodness-of-fit test. 


\section{Study design}

Two six-minute resting-state runs, during which participants were instructed to lie still, relax, and keep their eyes open, were part of a larger study investigating emotion dysregulation in BPD [van Zutphen et al., submitted]. Resting-state runs were acquired before and after an emotion regulation task, which was an adapted version of previously published emotional regulation paradigm (Koenigsberg et al., 2009; Ochsner, Bunge, Gross \& Gabrieli, 2002). In this task, participants were presented with negative, positive, erotic, and neutral pictures and instructed to either attend to the picture and respond naturally without altering their emotional state (passive viewing condition) (Ochsner et al., 2002), or to regulate their emotional state by realizing that they are safe (regulation condition), a technique inspired by schema therapy (Arntz \& van Genderen, 2009). Since BPD patients are particularly responsive to interpersonal stimuli (Koenigsberg et al., 2009), only pictures with a social content (i.e. one person emotionally relating to the viewer or two or more persons in interaction) were selected. Each trial consisted of a $2 \mathrm{~s}$ visual instruction to 'look' or 'realize being safe, 8 s presentation of the pictures while implementing the instruction, $4 \mathrm{~s}$ rating period and variable 5-6.5s fixation period (for schematic overview Figure S1). During the rating period, participants assessed their momentary emotional state on a horizontal visual analogue scale (-100 to $100 \mathrm{~mm}$ ). The order of the conditions and stimulus categories were equally divided and presented in a pseudo-randomized order. The task consisted of 96 trials divided into four runs of 24 trials each. The time between the two resting-state scans was about 45 minutes (two runs of the task, then an anatomical scan, next two more runs of the task). The experimental manipulation successfully affected the subjective ratings and brain activation [van Zutphen et al., submitted]. After the MRI data acquisition, participants evaluated the arousal and valence of each picture presented in the task, using the SelfAssessment Manikin (Bradley \& Lang, 1994). Furthermore, participants were asked to fill out an exit questionnaire, in which they stated their compliance to the study instructions. Finally, prior to the scanning session and immediately afterward participants rated their level of dissociative experiences with the Dissociation-Tension-Scale (Stiglmayr, Shapiro, Stieglitz, Limberger \& Bohus, 2001) as well as the level of anxiety and nervousness.

\section{FMRI data acquisition and statistical analyses}

Structural and functional MRI data were acquired with 3-Tesla scanners at each site. Participants were scanned in head first supine position. Head movements were minimized using foam paddings. Functional images were taken with a $\mathrm{T}^{*}$-weighted echo planar imaging (EPI) sequence with the following parameters: 180 volumes, $\mathrm{TR}=2000 \mathrm{~ms}$, TE $=$ $27 \mathrm{~ms}$, flip angle $=90^{\circ}$, FoV $=192 \times 192 \mathrm{~mm}$, voxel size $=3 \times 3 \times 3 \mathrm{~mm}$, and matrix $=64 \times$ 64. One volume in Maastricht consisted of 32, and in Freiburg and Lübeck of 34, interleaved 
measured axial slices. The T2*-weighted slices were optimized with a negative tilt of $30^{\circ}$, to minimize susceptibility and distortion artifacts within the amygdala (Morawetz et al., 2008) in Maastricht and Freiburg. Anatomical images were acquired with high-resolution T1-weighted sequence with the following parameters: TR $=2250 \mathrm{~ms}$, TE $=2.6 \mathrm{~ms}$, flip angle $=9^{\circ}$, FoV $=256 \times 256 \mathrm{~mm}$, voxel size $1 \times 1 \times 1 \mathrm{~mm}$. In total, 192 slices were obtained in Maastricht, 160 in Freiburg, and 170 in Lübeck. Scanner specifications and preprocessing steps are described in the supplementary material.

The statistical analyses were performed using BrainVoyager 2.8 (Brain Innovation, Maastricht, The Netherlands), SPSS Statistics 21 (IBM Corp, NY), NeuroElf (MR imaging analysis toolbox, www.neuroelf.net), and custom routines in Matlab (Mathworks, Natick, MA). An amygdala seed-based whole-brain correlation approach was used. The masks for left and right amygdala were obtained by applying a sphere of $5 \mathrm{~mm}$ radius around the coordinates (Tal: $\pm 22,-6,-14)$ which is similar to that used in the previous rsFC studies in NPC (Veer et al., 2011) and in BPD (Krause-Utz et al., 2014b). The amygdala seeds of the left and right hemisphere are depicted in Figure S2.

Functional connectivity was estimated with the Pearson $r$ correlation coefficient. The time courses of the two amygdala seeds were extracted, due to high correlations between the left and right seed averaged (Krause-Utz et al., 2014a; Krause-Utz et al., 2014b), and then correlated with the time course of all other voxels in the brain on the individual level. The resulting $r$ values were converted to z-scores using Fisher's transformation in order to increase normality of the distribution. The obtained z-scores were then entered into a second-level analysis. We attempted to equalize the scanner parameters across sites, but at the local sites not everything could be translated exactly the same; therefore, a discrete factor representing site was entered into a regression analysis to estimate the effect of site on the amygdala rsFC. The resulting residual amygdala rsFC data were used in the subsequent random effects analysis, thereby accounting for effects of site. Multiple comparisons across space were FWE corrected on a cluster-level, using Monte Carlo simulation (1000 iterations) based on the smoothness of a statistical map (Forman et al., 1995; Goebel, Esposito \& Formisano, 2006). Statistical maps were first thresholded at $p=.01$ and then corrected at the 3D cluster level at $p=.05$.

To determine whether the emotion regulation task led to differential changes in amygdala rsFC in BPD compared to NPC, we calculated a two-way mixed ANOVA and analyzed the time (before vs. after the task) $\times$ group (BPD vs. NPC) interaction. To avoid detection of 'spurious' clusters of interaction, we restricted the interpretation of the interaction F-map in the following way. First, in order to indicate whether there were any baseline group differences in the amygdala rsFC before the emotion regulation task, we performed two-tailed independent sample t-test with the contrast BPD vs. NPC. Second, to indicate whether there were significant changes in the rsFC of the amygdala after the 
task, we performed two-tailed paired t-test in each group with the contrast resting-state after vs. before the task. The t-map represents a putative change in the amygdala rsFC for each group, respectively. Following, we used a conjunction analysis of the two t-maps to determine whether there was any common network of the post-task change for both groups. With these restrictions, we limited our interpretation to only those interaction effects that were based on an effect of the task in at least one group regardless any potential baseline group differences. The individual average $z$-score (the strength of the rsFC) was extracted from the remaining clusters and exported to SPSS for analysis of simple effects to indicate the direction of a significant connectivity change in each group. Finally, each cluster was investigated post-hoc for the confounding effect of medication within the BPD group.

Further, we performed correlation analyses within the BPD group to test whether the differences in severity of BPD pathology and traumatic events in childhood were associated with the magnitude of change in the amygdala rsFC network representing differential posttask change in BPD compared to NPC. BPD pathology was indicated by individual scores of the BPDSI, BSI, BPD checklist, and the baseline level of dissociation measured with a four-item Dissociation-Tension-Scale. The severity of childhood abuse and neglect was expressed by a summation of the ITEC subscales. In addition, we assessed whether the change (increase) in the level of dissociation before vs. after scanning is associated with change in the amygdala rsFC to explore the impact of more state-dependent BPD characteristics. These scores were correlated with the magnitude of change in amygdala rsFC with the clusters retrieved from the group $\times$ time interaction. The magnitude was indicated by subtracting the $z$-scores of the resting-state before from the resting-state after the task, indicating the higher the $z$-score, the more increase in the rsFC after the task. Each correlational analysis was familywise error (FWE) corrected by Bonferroni procedure.

Lastly, we explored whether the results of altered changes in the amygdala rsFC after the emotion regulation task are specific for BPD or common with CCP. We performed a two-way mixed ANOVA in BPD vs. CCP, and analyzed the time $\times$ group interaction on the individual $z$-scores. We restricted the ANOVA model to the brain regions that previously indicated the significant interaction effect between BPD and NPC. The analysis was FWE corrected by Bonferroni procedure.

\section{Results}

\section{Behavioral results}

To determine whether each group implemented the task instructions and regulated their emotional state, the ratings of emotional state obtained immediately after the passive viewing or regulation condition across stimulus categories were compared between BPD 
and NPC. Both groups implemented the task instructions, as indicated by the significant change in their behavioral performance of the regulation condition, compared to the passive viewing condition [NPC: $M=16.67, \mathrm{SD}=41.81, t_{37}=2.46, p=.02$; BPD: $\mathrm{M}=11.90$, SD $\left.=31.41, t_{46}=2.60, p=.01\right]$. Additionally, the condition $\times$ group interaction was not significant $(p=.55)$, indicating that both groups regulated their emotional state to a similar extent between the two resting-state runs.

\section{Group differences in functional connectivity at baseline}

A common network of the intrinsic amygdala connectivity was obtained in both BPD and NPC before and after the emotion regulation task (Figure S3A), which was identified as similar to patterns previously described in the literature (e.g. Roy et al., 2009).

At baseline, BPD patients exhibited decreased amygdala rsFC with a cluster comprising the right ventral ACC and right orbitofrontal cortex [peak coordinates Tal: 11, 46, $0 ; t_{85}=3.89$; Figure 1]. No significant differences were obtained for the cluster between BPD patients who were medicated vs. non-medicated $(p=.85)$.

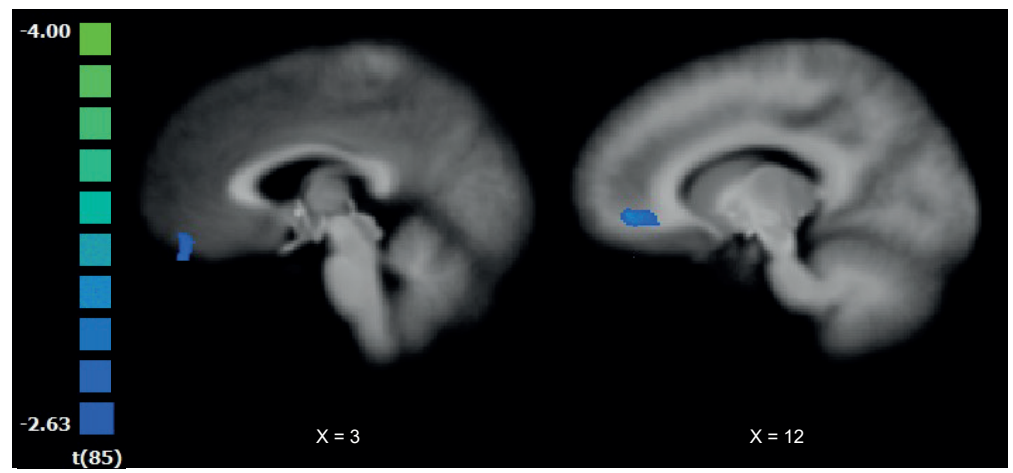

Figure 1. Baseline differences in the functional connectivity between BPD and NPC

The t-map was corrected at the cluster level $\left(p=.05 ; \mathrm{k}=675 \mathrm{~mm}^{3}\right)$ and overlaid on an anatomical image averaged over all participants in the Talairach standard space, according to the radiological convention. The cold colors indicate weaker resting-state functional connectivity of the amygdala in the BPD patients compared to the NPC group.

\section{Change in functional connectivity after the emotion regulation task}

After the emotion regulation task, NPC exhibited increased amygdala rsFC with the following areas: bilateral insula, striatum, superior frontal gyrus, middle frontal gyrus, ACC, lingual gyrus, right posterior cingulate cortex (PCC), medial PFC, posterior part of the left middle temporal gyrus, right precentral gyrus, right fusiform gyrus, and left cuneus (Figure 2A; Table 2). 

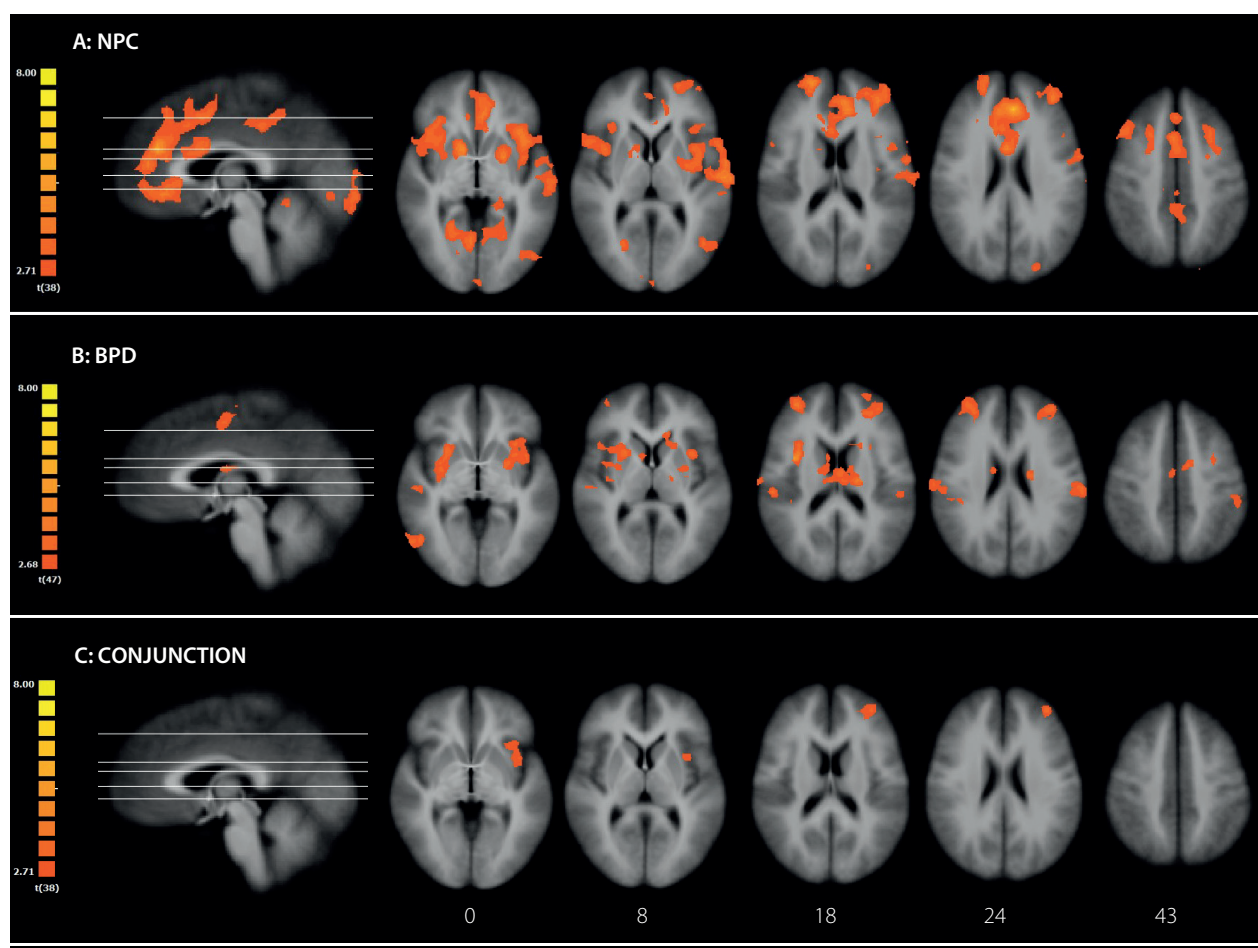

D:
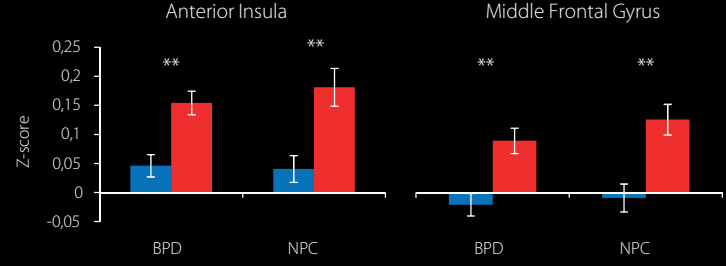

Before - After

\section{Figure 2. Change in the functional connectivity after the emotion regulation task}

Panel A depicts the change in the amygdala resting-state functional connectivity in the NPC group, whereas panel $\mathrm{B}$ depicts the change in BPD patients. Panel $\mathrm{C}$ depicts the results of the conjunction analysis to indicate $\mathrm{a}$ common network of the change in the amygdala resting-state functional connectivity for both groups. Panel $D$ depicts bar plots of the mean $z$-scores ( \pm SEM) of the clusters resulting from the conjunction analysis. The t-maps were corrected at the cluster level $\left(p=.05 ; \mathrm{k}=1134,891,837 \mathrm{~mm}^{3}\right.$ for the map of NPC, BPD, and conjunction, respectively) and overlaid on an anatomical image averaged over all participants in the Talairach standard space, according to the radiological convention. The hot colors indicate increased resting-state functional connectivity of the amygdala after the task. Numbers in the bottom row indicate $z$-coordinates of the axial sections in the Talairach standard space.

In BPD patients, we observed increased amygdala rsFC after the emotion regulation task with the following areas: bilateral insula, left caudate, bilateral middle frontal gyrus, posterior part of the right middle temporal gyrus, left postcentral gyrus, right supplementary motor area, and left superior and inferior parietal lobule (Figure 2B; Table 2). The time (before vs. after) 
$\times$ medication (medicated vs. non-medicated) interaction within this network did not yield significant results. None of the identified clusters overlapped with the cluster indicating baseline differences between BPD and NPC, which did not significantly change after the task ( $p>$.06, uncorrected).

Table 2. Change in the functional connectivity after the emotion regulation task.

\begin{tabular}{|c|c|c|c|c|c|c|c|}
\hline \multirow{2}{*}{ Region } & \multirow{2}{*}{$L / R$} & \multirow{2}{*}{ BA } & \multicolumn{3}{|c|}{$\begin{array}{c}\text { Peak voxel coordinates } \\
\text { (Talairach) }\end{array}$} & \multirow{2}{*}{$\begin{array}{l}\text { Size } \\
\left(\mathrm{mm}^{3}\right)\end{array}$} & \multirow{2}{*}{$t$} \\
\hline & & & $\mathrm{x}$ & y & z & & \\
\hline \multicolumn{8}{|l|}{ NPC } \\
\hline Caudate & $\mathrm{R}$ & - & 11 & 7 & 0 & 10394 & 4.79 \\
\hline Precentral Gyrus & $\mathrm{R}$ & 9 & 44 & 19 & 39 & 1932 & 3.87 \\
\hline Superior Frontal Gyrus & $\mathrm{R}$ & 10 & 20 & 58 & 18 & 3068 & 4.81 \\
\hline Fusiform Gyrus & $\mathrm{R}$ & 37 & 29 & -50 & -9 & 9590 & 4.97 \\
\hline Medial Frontal Gyrus & $\mathrm{R}$ & 32 & 20 & 10 & 45 & 2483 & 3.69 \\
\hline Anterior Cingulate Gyrus & $L$ & 32 & -4 & 37 & 24 & 21876 & 6.34 \\
\hline Posterior Cingulate Gyrus & $\mathrm{R}$ & 31 & 5 & -35 & 39 & 3282 & 4.42 \\
\hline Lingual Gyrus & $L$ & 18 & -1 & -92 & -6 & 1279 & 4.13 \\
\hline Culmen & $\mathrm{L}$ & - & -10 & -50 & -9 & 10382 & 5.22 \\
\hline Cuneus & L & 19 & -25 & -86 & 33 & 1395 & 4.40 \\
\hline Middle Frontal Gyrus & $\mathrm{L}$ & 10 & -37 & 43 & 21 & 5195 & 4.43 \\
\hline Insula & $L$ & 13 & -37 & 10 & 3 & 18351 & 5.10 \\
\hline Middle Frontal Gyrus & L & 8 & -25 & 16 & 39 & 4776 & 4.72 \\
\hline Middle Temporal Gyrus & $\mathrm{L}$ & 37 & -43 & -65 & 9 & 1682 & 3.88 \\
\hline \multicolumn{8}{|l|}{$\mathrm{BPD}$} \\
\hline Superior Temporal Sulcus & $\mathrm{R}$ & 22 & 44 & -26 & -6 & 3661 & 4.55 \\
\hline Middle Temporal Gyrus & $\mathrm{R}$ & 37 & 47 & -62 & 0 & 1269 & 3.79 \\
\hline Middle Frontal Gyrus & $\mathrm{R}$ & 10 & 32 & 49 & 18 & 4197 & 4.15 \\
\hline Insula & $\mathrm{R}$ & 13 & 35 & 4 & 19 & 6486 & 5.44 \\
\hline Caudate & $\mathrm{L}$ & - & -20 & -14 & 21 & 2932 & 5.04 \\
\hline Supplementary Motor Area & $\mathrm{R}$ & 6 & 2 & -8 & 48 & 1679 & 3.83 \\
\hline Insula & $\mathrm{L}$ & 13 & -31 & 10 & 12 & 6027 & 5.31 \\
\hline Superior Parietal Lobule & $\mathrm{L}$ & 7 & -19 & -56 & 60 & 2429 & 4.43 \\
\hline Sub-Gyral & $L$ & 6 & -25 & -2 & 57 & 1081 & 3.66 \\
\hline Middle Frontal Gyrus & $L$ & 10 & -31 & 46 & 21 & 2665 & 3.93 \\
\hline Inferior Parietal Lobule & $L$ & 40 & -52 & -32 & 36 & 1160 & 3.99 \\
\hline Postcentral Gyrus & $L$ & 2 & -61 & -20 & 24 & 1029 & 3.89 \\
\hline \multicolumn{8}{|l|}{ Conjunction } \\
\hline Middle Frontal Gyrus & $L$ & 10 & -34 & 46 & 21 & 1238 & 3.76 \\
\hline Insula & $\mathrm{L}$ & 13 & -34 & 7 & 6 & 1232 & 3.48 \\
\hline
\end{tabular}

Statistical maps (resting-state after vs. before the task) are corrected at the cluster level $\left(p=.05 ; \mathrm{k}=1134,891,837 \mathrm{~mm}^{3}\right.$ for the NPC, BPD, and conjunction map, respectively). Anatomical labels of the peak voxel coordinates are identified with the "nearest gray matter" option in the Talairach Client (www.talairach.org) (Lancaster et al. 2000). BA = Brodmann area; $R=$ right hemisphere; $L=$ left hemisphere. 
The conjunction analysis showed similar increases after versus before the emotion regulation task in the amygdala rsFC with the left middle frontal gyrus and left anterior insula in both groups (Figure 2C and 2D; Table 2).

These results indicate that the emotion regulation task induced changes in the pattern of amygdala rsFC in both groups, and a common network of post-task changes comprised increased amygdala rsFC with the middle frontal gyrus and anterior insula.

\section{Change in functional connectivity in BPD and NPC after the emotion regulation task}

The resulting F-map revealed a significant group $\times$ time interaction in the following clusters: the right superior and left inferior frontal gyrus, left superior and inferior temporal gyrus, medial PFC, left PCC, right cuneus, and left superior parietal lobule (Figure 3A; Table 3). The clusters of the cuneus and inferior temporal gyrus were not analyzed further, because they did not show overlap with the t-map of either BPD or NPC.

Simple effects on the $z$-scores extracted from the clusters indicated specific group differences in the change of the amygdala rsFC after the task (Figure 3B). After the task, NPC showed an increase in the amygdala rsFC with all clusters except for the left superior parietal lobule. In contrast to NPC, BPD patients exhibited decreased post-task amygdala rsFC with PCC and increased rsFC with the left superior parietal lobule. None of these clusters overlapped with the cluster indicating baseline differences between BPD and NPC, of which the non-significant interaction effect was confirmed in the post-hoc analysis ( $p$ > .69 , uncorrected). No significant time $\times$ medication interaction within the BPD group was observed for any of the clusters ( $p>.06$, uncorrected). Taken together, these results indicate that BPD patients and NPC showed different post-task patterns of the amygdala rsFC.

Table 3. Differential changes in the functional connectivity between BPD and NPC after the emotion regulation task.

\begin{tabular}{|c|c|c|c|c|c|c|c|}
\hline \multirow{2}{*}{ Region } & \multirow{2}{*}{$L / R$} & \multirow{2}{*}{ BA } & \multicolumn{3}{|c|}{ Peak voxel coordinates (Talairach) } & \multirow{2}{*}{ Size $\left(\mathrm{mm}^{3}\right)$} & \multirow{2}{*}{$F$} \\
\hline & & & $\mathbf{x}$ & $\mathbf{y}$ & $\mathbf{z}$ & & \\
\hline Superior Frontal Gyrus & $\mathrm{R}$ & 6 & 17 & 22 & 60 & 748 & 11.87 \\
\hline Cuneus & $\mathrm{R}$ & 19 & 20 & -83 & 30 & 662 & 10.63 \\
\hline (Posterior) Cingulate Gyrus & $\mathrm{L}$ & 31 & -1 & -32 & 39 & 2650 & 14.71 \\
\hline Medial Prefrontal Gyrus & $\mathrm{R}$ & - & 2 & 49 & 3 & 2271 & 14.67 \\
\hline Superior Parietal Lobule & $L$ & 7 & -16 & -56 & 63 & 1290 & 15.08 \\
\hline Inferior Frontal Gyrus & $L$ & 45 & -58 & 13 & 21 & 481 & 11.64 \\
\hline Superior Temporal Gyrus & L & 42 & -67 & -13 & 9 & 544 & 12.74 \\
\hline Inferior Temporal Gyrus & $\mathrm{L}$ & 20 & -61 & -23 & -24 & 484 & 12.97 \\
\hline
\end{tabular}

Statistical maps are corrected at the cluster level $\left(p=.05 ; \mathrm{k}=459 \mathrm{~mm}^{3}\right)$. Anatomical labels of the peak voxel coordinates are identified with the "nearest gray matter" option in the Talairach Client (www.talairach.org) (Lancaster et al. 2000). $\mathrm{BA}=$ Brodmann area; $\mathrm{R}=$ right hemisphere; $\mathrm{L}=$ left hemisphere. 


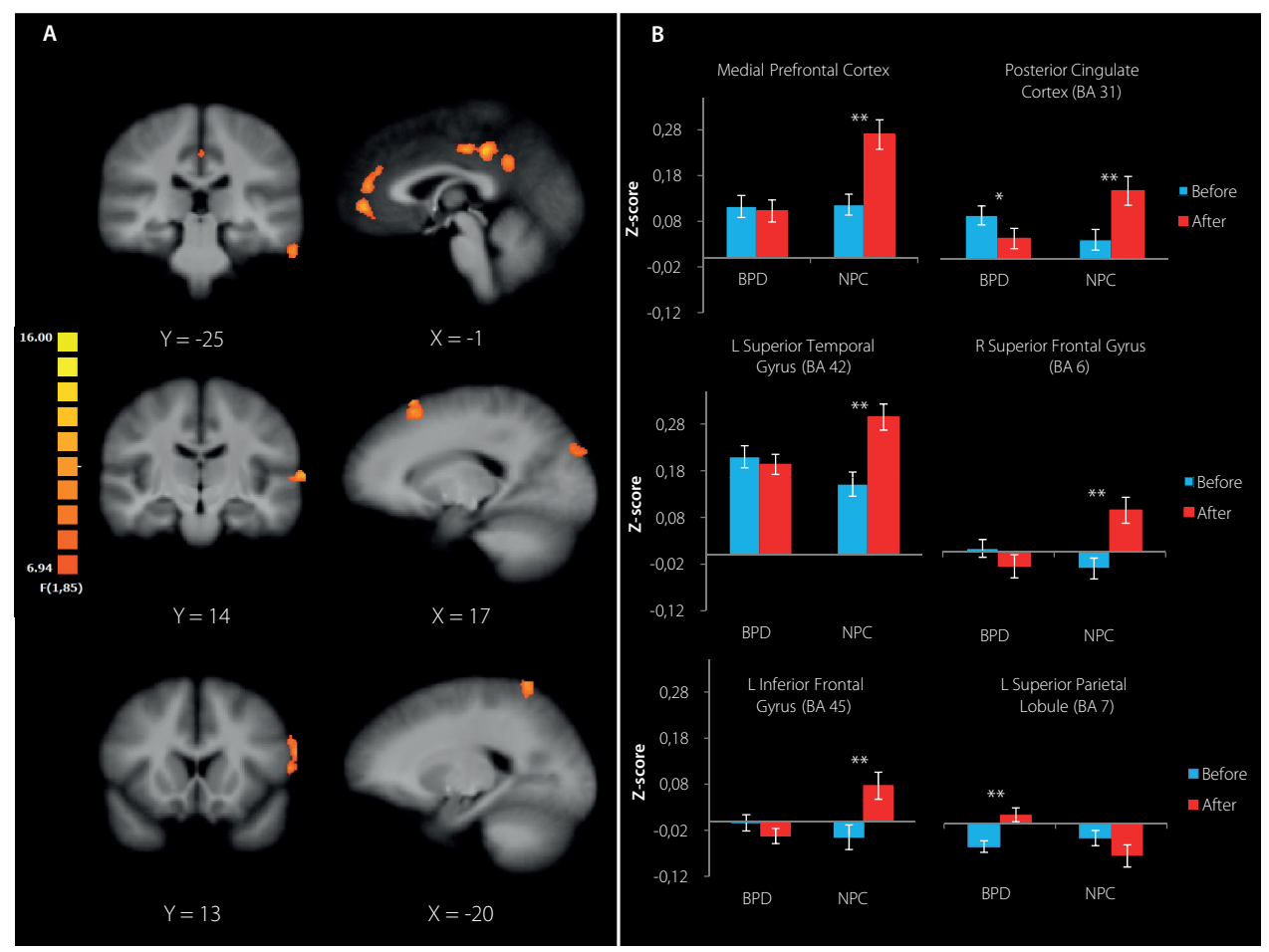

Figure 3. Changes in the functional connectivity in BPD and NPC after the emotion regulation task

Panel A depicts the F-map of the time $\times$ group interaction (corrected at the cluster level, $p=.05 ; \mathrm{k}=459 \mathrm{~mm}^{3}$ ) and overlaid on an anatomical image averaged over all participants in the Talairach standard space, according to the radiological convention. Panel B depicts the bar plots of the mean z-scores ( \pm SEM) in each group before and after the emotion regulation task and indicates significant within-group difference. The z-scores were extracted from six out of eight significant clusters (see Methods). The between-group comparisons after the task were significant in all clusters ( $p s<.01)$, and no group differences were shown before the task.

\section{Change in the functional connectivity and BPD characteristics}

The correlation analysis did not show significant associations between the severity of BPD pathology, including baseline level of dissociation, and any of the clusters retrieved from the group $\times$ time interaction. Subsequently, we tested the hypothesis that BPD characteristics are not associated with changes in the network of differential effects between BPD and NPC, but rather with the network that showed a putative response for BPD patients. Therefore, we performed a whole-brain correlation analysis with the mask of the network that showed significantly increased post-task amygdala rsFC in BPD patients. Again, we did not observe significant results for the BSI, BPD checklist or baseline level of dissociation. We, however, observed a positive association between BPDSI and the magnitude of change in the amygdala rsFC with a cluster corresponding to the left middle frontal gyrus $[r(46)=$ 
45, $p=.001$; peak coordinates Tal: $-25,43,18$; Figure 4A]. We repeated the same analytical strategy for the correlation analysis with the ITEC. Again, no significant association was found between ITEC and any of the clusters of the group $\times$ time interaction. When using the mask of post-task amygdala rsFC network observed in BPD, we found a negative association of the ITEC with the magnitude of change in the amygdala rsFC with the ventral part of the left insula $[r(46)=-.52, p<.001$; peak coordinates Tal: $-34,-2,-2$; Figure 4B].

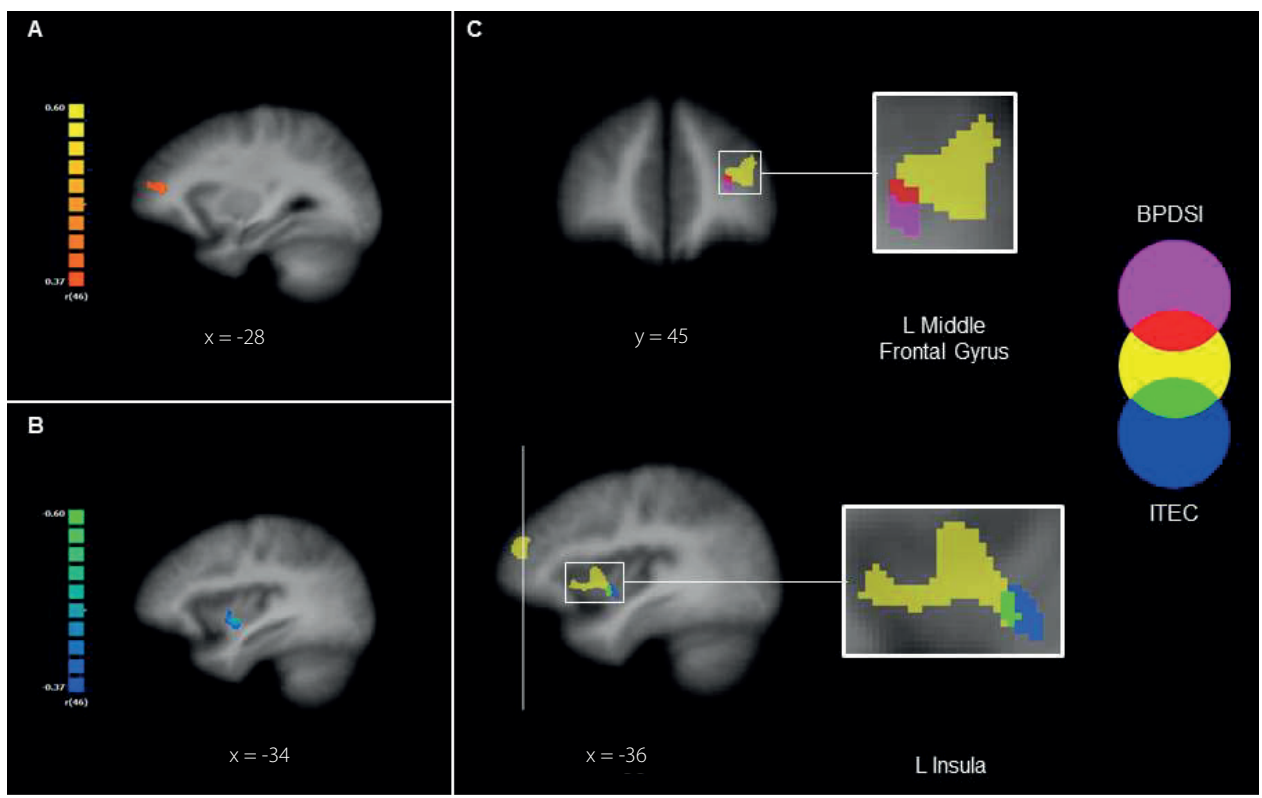

Figure 4. Correlational analyses of the change in the functional connectivity and BPD

\section{characteristics}

Panel A depicts the correlation analysis between the magnitude of change in the amygdala resting-state functional connectivity after the task and the severity of the BPD psychopathology (BPDSI). Panel B depicts the correlation analysis with the severity of the reported childhood traumatic events (ITEC). The r-maps were corrected at the cluster level $\left(p=.05 ; \mathrm{k}=270 \mathrm{~mm}^{3}\right.$ and $351 \mathrm{~mm}^{3}$ for the map of BPDSI and ITEC, respectively) and restricted to the mask of the network that showed significantly increased resting-state functional connectivity of the amygdala after the task in the BPD group (see Methods). Positive association is shown in hot colors whereas cold colors indicate negative association. In Panel C the two r-maps were overlaid with the map of the conjunction analysis (see Methods), which is depicted in yellow. Statistical maps were overlaid on an anatomical image averaged over all participants in the Talairach standard space, according to the radiological convention.

The two clusters showed minimal overlap with the clusters of the left middle frontal gyrus and the left insula that were retrieved from our conjunction analysis representing a common network of post-task changes of the BPD and NPC (Figure 4C). These findings suggest that the severity of BPD psychopathology and childhood traumatic are associated with the changes of the intrinsic amygdala connectivity that are putative for BPD but not for NPC. 
Increase in the level of dissociation in BPD patients after versus before scanning was positively associated with the change of coupling between the amygdala and two clusters retrieved from the interaction: inferior frontal gyrus $(r=.34, p=.025)$ and superior temporal gyrus ( $r=.33, p=.030$ ), demonstrating in BPD stronger amygdala rsFC with more change in dissociation. However, these results did not survive Bonferroni correction. Hence, similarly to the previous analyses, we performed whole-brain analysis with the mask of post-task amygdala rsFC network observed in BPD. We did not observe any significant results. These findings suggest that in BPD patients increased level of dissociation after scanning does not significantly contribute to their altered change of amygdala rsFC.

\section{Diagnosis-specificity: comparison with Cluster-C personality disorders patients}

From the behavioral measures, we observed a weak trend toward a significant difference between the passive viewing and regulation condition across stimulus categories in CCP patients $\left(M=11.74, \mathrm{SD}=28.46, t_{20}=1.89, p=.07\right)$. There was no significant difference between CCP and BPD ( $p=.98)$ as well as NPC ( $p=.63)$ in the difference between the passive viewing and regulation condition, which suggests that the difference score is similar in magnitude and failed to reach significance in CCP likely due to smaller number of participants. The amygdala rsFC network observed in CCP before and after the task was similar to the patterns observed in the BPD and NPC (Figure S3B). The interaction analysis between BPD and CCP patients did not yield significant results after Bonferroni correction. We additionally performed a conjunction analysis of the whole-brain F-maps indicating the interaction effects BPD vs. NPC and CCP vs. NPC to explore whether there were common altered changes in the amygdala rsFC in both patients groups. Again, no significant results were observed. However, the uncorrected map (thresholded at $p=.01$ ) showed a cluster in the left vmPFC (peak coordinates Tal: -4, 43, -3). We used the z-scores extracted from the cluster in the subsequent post-hoc analysis, using two-way mixed ANOVA with three groups and observed a significant time $\times$ group interaction $(p=.001)$. Only NPC exhibited a significant post-task increase in the amygdala rsFC with the cluster $\left[t_{38}=3.86 ; p<.001\right]$. The exploratory results suggest that effortful emotion regulation leads to an altered amygdala rsFC with the vmPFC that might be common for personality disorders characterized by emotional problems.

\section{Discussion}

In the present study, we investigated whether a cognitive emotion regulation task induces different changes of the amygdala rsFC with the PFC in BPD patients, compared to NPC. Although according to the behavioral results both groups reported similar change in their 
emotional state due to cognitive regulation, BPD patients exhibited a different post-task amygdala rsFC, which did not involve the brain network of pre-task differences between the groups. While NPC showed increased post-task amygdala rsFC with the medial, dorsolateral and ventrolateral PFC, and superior temporal gyrus, BPD patients exhibited a lack of change in this network. Compared to NPC, BPD patients surprisingly showed decreased post-task amygdala rsFC with the PCC and increased rsFC with the superior parietal lobule.

Effortful emotion regulation is a complex cognitive process that involves neuronal systems responsible for emotion generation and regulation (Etkin, Buchel \& Gross, 2015; Ochsner et al., 2012). The amygdala is a key node detecting potential threats (Phelps \& LeDoux, 2005; Phillips, Drevets, Rauch \& Lane, 2003) and together with the striatum, anterior insula and dACC forms a salience network (Menon, 2015), which selects stimuli as relevant for current goals and thus initiates subsequent regulatory mechanisms subserved by a fronto-parietal cognitive control network (Ochsner et al., 2009). We observed in NPC that our emotion regulation task induced changes in the amygdala rsFC patterns that involved both the emotion generating and regulatory networks. Hence, we infer that such enduring changes reflect employment of neuronal circuits, which subserve the complete process of effortful emotion regulation including the generation (i.e. effects of passive viewing or presentation of different stimulus categories) and regulation of emotions, as these components could not be strictly disentangled.

In light of this, BPD patients seem to modulate their emotions with different brain networks. They showed altered post-task amygdala rsFC with the dIPFC, vIPFC, and temporal cortex that comprise the cognitive control network used to maintain, select, evaluate, and reinterpret the emotion-related representations retrieved from the lateral temporal cortex (Buhle et al., 2014; Ochsner et al., 2012). Moreover, when BPD patients are confronted with the effortful emotion regulation task, they exhibit additional post-task cross-talk between the amygdala and superior parietal lobule, which is implicated in attention (Corbetta \& Shulman, 2002; Han et al., 2004) and episodic memory retrieval (Cabeza, Ciaramelli, Olson \& Moscovitch, 2008). Additionally, the more severe the condition of BPD according to the BPDSI, the more areas of the left middle frontal gyrus within the post-task intrinsic amygdala network was observed. We speculate that severely affected BPD patients engaged more the middle frontal gyrus to maintain their reappraisals in working memory (Buhle et al., 2014; Kohn et al., 2014) due to following reasons. Over the course of the task, the passive viewing condition can induce (un-)intentional regulation in patients who are emotionally hyperreactive (e.g. Donegan et al., 2003; Herpertz et al., 2001; Koenigsberg et al., 2014) and/ or the regulation cannot be terminated by the impaired inhibitory loop, which involves the VIPFC, when a goal-appropriate reappraisal has been selected (Morawetz, Bode, Baudewig, Kirilina \& Heekeren, 2015). To overcome this deficit, BPD patients might employ a compensatory but maladaptive strategy, such as dissociation, which typically occur due to 
overwhelming emotions in stressful situations (Lanius et al., 2010). While previous research shows that dissociation is associated with rsFC and predicts changes in brain activity due to emotional tasks (Krause-Utz et al., 2014a; Krause-Utz et al., 2014b; Wolf et al., 2011), in the current study, we did not find evidence for similar associations. Yet, these associations might become apparent when BPD patients encounter emotional challenge. To this end, we additionally explored whether increased dissociation after scanning is associated with differential change of the rsFC in BPD. Although this additional analysis did not survive correction for multiple comparisons, it is noteworthy for future studies to report that increased dissociation was moderately associated with increased amygdala rsFC with the inferior frontal gyrus and superior temporal gyrus. While these findings might potentially indicate a tendency for a compensatory mechanism in BPD, they are speculative and should be taken with caution.

It is assumed that the cognitive control network of effortful emotion regulation modulates the amygdala activity indirectly via the vmPFC (Motzkin, Philippi, Wolf, Baskaya \& Koenigs, 2015; Ochsner et al., 2012; Urry et al., 2006). The amygdala is extensively interconnected with the mPFC (Ghashghaei, Hilgetag \& Barbas, 2007) and its FC strength predicts lower levels of anxiety and effective emotion regulation (Kim et al., 2011). Previously, Kamphausen and colleagues (2013) reported that an exaggerated amygdala response to threat in BPD patients is associated with a failure of the regulatory amygdala-mPFC connectivity loop. Similarly, the present results show that enduring effects of emotion regulation in BPD are absent in the amygdala-mPFC circuit. The plausible dysfunction of this network may underlie impaired cognitive emotion regulation in BPD patients. Although the altered neurocognitive component of effortful emotion regulation might be specific for BPD (Das et al., 2014), our results suggest that the direct regulation of the amygdala by vmPFC seems to be common for other personality disorders characterized by emotional problems.

Effortful emotion regulation induced altered post-task changes of the intrinsic amygdala connectivity with the PCC, which we did not expect. The PCC is a key node in the default mode network (DMN) and has been implicated in self-referential processing and autobiographical memory (Buckner \& Carroll, 2007; Cavanna \& Trimble, 2006). Veer and colleagues (2011) reported increased amygdala rsFC with PCC and the adjacent precuneus after acute social stress in NPC, which after the task might promote the evaluation of emotionally salient events stored in autobiographical memory to prepare for future challenges. Individuals who experienced childhood maltreatment may not be able to benefit from such an adaptive strategy (van derWerffet al., 2013). Childhood abuse and neglect is prevalent in BPD patients (Zanarini et al., 2002) and contributes to their emotion dysregulation (Fernando et al., 2014). BPD patients also show evidence for altered self-referential processing at rest (KrauseUtz et al., 2014b; Wolf et al., 2011) and during pain, which is associated with dissociation (Kluetsch et al., 2012), likely influenced by childhood traumatization (Vermetten \& Spiegel, 
2014). Although we did not find the severity of early-life abuse and neglect in BPD patients to be associated with post-task changes in the amygdala rsFC with the PCC, a negative association was observed in the left insula, a brain region implicated in somatosensory processing and interoception (Craig, 2009) and responsible for switching between the DMN and fronto-parietal control network (Uddin, 2015). Hence, we speculate that BPD patients tend to avoid self-related appraisals of salient emotional stimuli, which might be partly mediated by their blunted affect caused by childhood maltreatment. Future studies could directly investigate brain connectivity of the intertwined relations between emotion, autobiographical memory, and childhood abuse and neglect in BPD (Jones et al., 1999; Winter, Elzinga \& Schmahl, 2014).

\section{Limitations}

In the current study, we included only females, which hamper generalizability to males. Second, the BPD patients represented a rather heterogeneous group given the presence of co-occurring disorders, with most prominent depression and substance abuse. Comorbid Axis I disorders are typical in BPD, and BPD patients without Axis I co-occurring disorders are rare and not representative for the disorder. As a consequence, we cannot exclude the possibility that our results might be affected by these comorbidities. Furthermore, lefthanded participants were included in the study. A third limitation of our study pertains to the possible impact of medication intake, which might have influenced the intrinsic organization of connectivity networks (Phillips, Travis, Fagiolini \& Kupfer, 2008). However, to recruit a representative and severe clinical sample we did not exclude patients on medication. We performed additional analyses within the BPD group, medicated vs. nonmedicated, to exclude acute effects of medication on our results, as adding medication as a covariate could remove some substantial part of the variance associated with group differences. Fourth, we have reported all results at a relatively lenient initial threshold of $p<$ .01 and corrected for multiple comparisons at cluster level at $p<.05$. However, to limit the possibility of false positives we restricted the interpretation of only those interaction effects that were based on an effect of the task in at least one group regardless potential group differences. Finally, we should always be cautious about the interpretation of the amygdala FC, because the blood-oxygenation level-dependent signal of the amygdala is susceptible to physiological confounds due to its proximity to draining veins. Of note, the results of the correlation analyses should be interpreted with some caution, because of a small sample size. 


\section{Conclusion}

In BPD patients, the emotion regulation task failed to increase amygdala intrinsic FC with brain regions essential for effortful emotion regulation, which suggests: (a) altered cognitive control typically used to indirectly alleviate distress by reinterpreting the meaning of emotional stimuli; (b) impaired direct regulation of emotional responses, which might be common for personality disorders; (c) avoidance of self-related appraisals induced by social emotional stimuli. The lack of enduring post-task effects in BPD patients in these networks might hamper their cognitive control over subsequent emotional challenge. These findings portray a complex picture and vicious circle of emotion dysregulation in BPD. 


\section{References}

American Psychiatric Association. (2013). Diagnostic and Statistical Manual of Mental Disorders (5th ed.). Arlengtion, VA: American Psychiatric Association.

Arntz, A., \& Dreessen, L. (1995). BPD-Klachtenlijst 47 [BPD Checklist]. The Netherlands: Maastricht University.

Arntz, A., van den Hoorn, M., Cornelis, J., Verheul, R., van den Bosch, W. M., \& de Bie, A. J. (2003). Reliability and validity of the borderline personality disorder severity index. Journal of Personality Disorders, 17, 45-59. doi: 10.1521/ pedi.17.1.45.24053

Arntz, A., \& van Genderen, H. (2009). Schema therapy for borderline personality disorder. Chichester: Wiley.

Banks, S. J., Eddy, K. T., Angstadt, M., Nathan, P. J., \& Phan, K. L. (2007). Amygdala-frontal connectivity during emotion regulation. Social Cognitive and Affective Neuroscience, 2, 303-312. doi: 10.1093/scan/nsm029

Bradley, M. M., \& Lang, P. J. (1994). Measuring emotion: the Self-Assessment Manikin and the Semantic Differential. Journal of Behavior Therapy and Experimental Psychiatry, 25, 49-59. doi: 10.1016/0005-7916(94)90063-9

Buckner, R. L., \& Carroll, D. C. (2007). Self-projection and the brain. Trends in Cognitive Sciences, 11, 49-57. doi: 10.1016/j.tics.2006.11.004

Buhle, J. T., Silvers, J. A., Wager, T. D., Lopez, R., Onyemekwu, C., Kober, H., . . Ochsner, K. N. (2014). Cognitive reappraisal of emotion: a meta-analysis of human neuroimaging studies. Cerebral Cortex, 24, 2981-2990. doi: 10.1093/cercor/bht154

Cabeza, R., Ciaramelli, E., Olson, I. R., \& Moscovitch, M. (2008). The parietal cortex and episodic memory: an attentional account. Nature Reviews. Neuroscience, 9, 613-625. doi: 10.1038/nrn2459

Carpenter, R. W., \& Trull, T. J. (2013). Components of emotion dysregulation in borderline personality disorder: a review. Current Psychiatry Reports, 15, 335. doi: 10.1007/s11920-012-0335-2

Cavanna, A. E., \& Trimble, M. R. (2006). The precuneus: a review of its functional anatomy and behavioural correlates. Brain, 129, 564-583. doi: 10.1093/brain/awl004

Corbetta, M., \& Shulman, G. L. (2002). Control of goal-directed and stimulus-driven attention in the brain. Nature Reviews. Neuroscience, 3, 201-215. doi: 10.1038/nrn755

Craig, A. D. (2009). How do you feel--now? The anterior insula and human awareness. Nature Reviews. Neuroscience, 10, 59-70. doi: 10.1038/nrn2555

Cremers, H. R., Veer, I. M., Spinhoven, P., Rombouts, S. A., Yarkoni, T., Wager, T. D., \& Roelofs, K. (2015). Altered corticalamygdala coupling in social anxiety disorder during the anticipation of giving a public speech. Psychological Medicine, 45, 1521-1529. doi: 10.1017/S0033291714002657

Cullen, K. R., Vizueta, N., Thomas, K. M., Han, G. J., Lim, K. O., Camchong, J., .. S Schulz, S. C. (2011). Amygdala functional connectivity in young women with borderline personality disorder. Brain Connectivity, 1, 61-71. doi: 10.1089/ brain.2010.0001

Das, P., Calhoun, V., \& Malhi, G. S. (2014). Bipolar and borderline patients display differential patterns of functional connectivity among resting state networks. Neurolmage, 98, 73-81. doi: 10.1016/j.neuroimage.2014.04.062

Derogatis, L. R. (1993). BSI Brief Symptom Inventory: Administration, Scoring, and Procedure Manual (4th ed.). Minneapolis, MN: National Computer Systems.

Doll, A., Sorg, C., Manoliu, A., Woller, A., Meng, C., Forstl, H., ... Riedl, V. (2013). Shifted intrinsic connectivity of central executive and salience network in borderline personality disorder. Frontiers in Human Neuroscience, 7, 727. doi: 10.3389/fnhum.2013.00727

Donegan, N. H., Sanislow, C. A., Blumberg, H. P., Fulbright, R. K., Lacadie, C., Skudlarski, P., ... Wexler, B. E. (2003). Amygdala hyperreactivity in borderline personality disorder: implications for emotional dysregulation. Biological Psychiatry, 54, 1284-1293.

Etkin, A., Buchel, C., \& Gross, J. J. (2015). The neural bases of emotion regulation. Nature Reviews. Neuroscience, 16, 693-700. doi: 10.1038/nrn4044

Fernando, S. C., Beblo, T., Schlosser, N., Terfehr, K., Otte, C., Lowe, B., . . Wingenfeld, K. (2014). The impact of selfreported childhood trauma on emotion regulation in borderline personality disorder and major depression. Journal of Trauma \& Dissociation, 15, 384-401. doi: 10.1080/15299732.2013.863262

Forman, S. D., Cohen, J. D., Fitzgerald, M., Eddy, W. F., Mintun, M. A., \& Noll, D. C. (1995). Improved assessment of significant activation in functional magnetic resonance imaging (fMRI): use of a cluster-size threshold. Magnetic Resonance in Medicine, 33, 636-647. 
Ghashghaei, H. T., Hilgetag, C. C., \& Barbas, H. (2007). Sequence of information processing for emotions based on the anatomic dialogue between prefrontal cortex and amygdala. Neurolmage, 34, 905-923. doi: 10.1016/j. neuroimage.2006.09.046

Giesen-Bloo, J. H., Wachters, L. M., Schouten, E., \& Arntz, A. (2010). The Borderline Personality Disorder Severity IndexIV: psychometric evaluation and dimensional structure. Personality and Individual Differences, 49, 136-141. doi: DOI 10.1016/j.paid.2010.03.023

Goebel, R., Esposito, F., \& Formisano, E. (2006). Analysis of functional image analysis contest (FIAC) data with brainvoyager QX: From single-subject to cortically aligned group general linear model analysis and selforganizing group independent component analysis. Human Brain Mapping, 27, 392-401. doi: 10.1002/ hbm.20249

Gordon, E. M., Breeden, A. L., Bean, S. E., \& Vaidya, C. J. (2014). Working memory-related changes in functional connectivity persist beyond task disengagement. Human Brain Mapping, 35, 1004-1017. doi: 10.1002/ hbm.22230

Grigg, O., \& Grady, C. L. (2010). Task-related effects on the temporal and spatial dynamics of resting-state functional connectivity in the default network. PLoS One, 5, e13311. doi: 10.1371/journal.pone.0013311

Han, S., Jiang, Y., Gu, H., Rao, H., Mao, L., Cui, Y., \& Zhai, R. (2004). The role of human parietal cortex in attention networks. Brain, 127, 650-659. doi: 10.1093/brain/awh071

Herpertz, S. C., Dietrich, T. M., Wenning, B., Krings, T., Erberich, S. G., Willmes, K., . . Sass, H. (2001). Evidence of abnormal amygdala functioning in borderline personality disorder: a functional MRI study. Biological Psychiatry, 50, 292-298.

Hutchison, R. M., Womelsdorf, T., Allen, E. A., Bandettini, P. A., Calhoun, V. D., Corbetta, M., ... Chang, C. (2013). Dynamic functional connectivity: promise, issues, and interpretations. Neurolmage, 80, 360-378. doi: 10.1016/j. neuroimage.2013.05.079

Jones, B., Heard, H., Startup, M., Swales, M., Williams, J. M., \& Jones, R. S. (1999). Autobiographical memory and dissociation in borderline personality disorder. Psychological Medicine, 29, 1397-1404.

Kamphausen, S., Schroder, P., Maier, S., Bader, K., Feige, B., Kaller, C. P., . . Tüscher, O. (2013). Medial prefrontal dysfunction and prolonged amygdala response during instructed fear processing in borderline personality disorder. The World Journal of Biological Psychiatry, 14, 307-318, S301-304. doi: 10.3109/15622975.2012.665174

Kim, M. J., Loucks, R. A., Palmer, A. L., Brown, A. C., Solomon, K. M., Marchante, A. N., \&Whalen, P. J. (2011). The structural and functional connectivity of the amygdala: from normal emotion to pathological anxiety. Behavioural Brain Research, 223, 403-410. doi: 10.1016/j.bbr.2011.04.025

Kluetsch, R. C., Schmahl, C., Niedtfeld, I., Densmore, M., Calhoun, V. D., Daniels, J., ... Lanius, R. A. (2012). Alterations in default mode network connectivity during pain processing in borderline personality disorder. Archives of General Psychiatry, 69, 993-1002. doi: 10.1001/archgenpsychiatry.2012.476

Koenigsberg, H. W., Denny, B. T., Fan, J., Liu, X., Guerreri, S., Mayson, S. J., .. . Siever, L. J. (2014). The neural correlates of anomalous habituation to negative emotional pictures in borderline and avoidant personality disorder patients. The American Journal of Psychiatry, 171, 82-90. doi: 10.1176/appi.ajp.2013.13070852

Koenigsberg, H. W., Fan, J., Ochsner, K. N., Liu, X., Guise, K. G., Pizzarello, S., .. Siever, L. J. (2009). Neural correlates of the use of psychological distancing to regulate responses to negative social cues: a study of patients with borderline personality disorder. Biological Psychiatry, 66, 854-863. doi: 10.1016/j.biopsych.2009.06.010

Kohn, N., Eickhoff, S. B., Scheller, M., Laird, A. R., Fox, P. T., \& Habel, U. (2014). Neural network of cognitive emotion regulation--an ALE meta-analysis and MACM analysis. Neurolmage, 87, 345-355. doi: 10.1016/j. neuroimage.2013.11.001

Krause-Utz, A., Elzinga, B. M., Oei, N. Y., Paret, C., Niedtfeld, I., Spinhoven, P., . . Schmahl, C. (2014a). Amygdala and dorsal anterior cingulate connectivity during an emotional working memory task in borderline personality disorder patients with interpersonal trauma history. Frontiers in Human Neuroscience, 8, 848. doi: 10.3389/ fnhum.2014.00848

Krause-Utz, A., Veer, I. M., Rombouts, S. A., Bohus, M., Schmahl, C., \& Elzinga, B. M. (2014b). Amygdala and anterior cingulate resting-state functional connectivity in borderline personality disorder patients with a history of interpersonal trauma. Psychological Medicine, 44, 2889-2901. doi: 10.1017/S0033291714000324

Krause-Utz, A., Winter, D., Niedtfeld, I., \& Schmahl, C. (2014C). The latest neuroimaging findings in borderline personality disorder. Current Psychiatry Reports, 16, 438. doi: 10.1007/s11920-014-0438-z 
Kroger, C., Vonau, M., Kliem, S., Roepke, S., Kosfelder, J., \& Arntz, A. (2013). Psychometric properties of the German version of the borderline personality disorder severity index--version IV. Psychopathology, 46, 396-403. doi: $10.1159 / 000345404$

Lanius, R. A., Vermetten, E., Loewenstein, R. J., Brand, B., Schmahl, C., Bremner, J. D., \& Spiegel, D. (2010). Emotion modulation in PTSD: clinical and neurobiological evidence for a dissociative subtype. The American Journal of Psychiatry, 167, 640-647. doi: 10.1176/appi.ajp.2009.09081168

Lenzenweger, M. F., Lane, M. C., Loranger, A. W., \& Kessler, R. C. (2007). DSM-IV personality disorders in the National Comorbidity Survey Replication. Biological Psychiatry, 62, 553-564. doi: 10.1016/j.biopsych.2006.09.019

Lewis, C. M., Baldassarre, A., Committeri, G., Romani, G. L., \& Corbetta, M. (2009). Learning sculpts the spontaneous activity of the resting human brain. Proceedings of the National Academy of Sciences of the United States of America, 106, 17558-17563. doi: 10.1073/pnas.0902455106

Linehan, M. M. (1993). Cognitive-Behavioral Treatment of Borderline Personality Disorder. New York: Guilford Press.

Lobbestael, J., Arntz, A., Harkema-Schouten, P., \& Bernstein, D. (2009). Development and psychometric evaluation of a new assessment method for childhood maltreatment experiences: the interview for traumatic events in childhood (ITEC). Child Abuse \& Neglect, 33, 505-517. doi: 10.1016/j.chiabu.2009.03.002

Menon, V. (2015). Salience Network. In A. W. Toga (Ed.), Brain mapping: an encyclopedic reference (pp. 597-611): Academic Press: Elsevier.

Morawetz, C., Bode, S., Baudewig, J., Kirilina, E., \& Heekeren, H. R. (2015). Changes in effective connectivity between dorsal and ventral prefrontal regions moderate emotion regulation. Cerebral Cortex. doi: 10.1093/cercor/ bhv005

Morawetz, C., Holz, P., Lange, C., Baudewig, J., Weniger, G., Irle, E., \& Dechent, P. (2008). Improved functional mapping of the human amygdala using a standard functional magnetic resonance imaging sequence with simple modifications. Magnetic Resonance Imaging, 26, 45-53. doi: 10.1016/j.mri.2007.04.014

Motzkin, J. C., Philippi, C. L., Wolf, R. C., Baskaya, M. K., \& Koenigs, M. (2015). Ventromedial prefrontal cortex is critical for the regulation of amygdala activity in humans. Biological Psychiatry, 77, 276-284. doi: 10.1016/j. biopsych.2014.02.014

Niedtfeld, I., Kirsch, P., Schulze, L., Herpertz, S. C., Bohus, M., \& Schmahl, C. (2012). Functional connectivity of painmediated affect regulation in borderline personality disorder. PLoS One, 7, e33293. doi: 10.1371/journal. pone.0033293

Ochsner, K. N., Bunge, S. A., Gross, J. J., \& Gabrieli, J. D. (2002). Rethinking feelings: an fMRI study of the cognitive regulation of emotion. Journal of Cognitive Neuroscience, 14, 1215-1229. doi: 10.1162/089892902760807212

Ochsner, K. N., Ray, R. R., Hughes, B., McRae, K., Cooper, J. C., Weber, J., ... Gross, J. J. (2009). Bottom-up and top-down processes in emotion generation: common and distinct neural mechanisms. Psychological Science, 20, 13221331. doi: 10.1111/j.1467-9280.2009.02459.x

Ochsner, K. N., Silvers, J. A., \& Buhle, J. T. (2012). Functional imaging studies of emotion regulation: a synthetic review and evolving model of the cognitive control of emotion. Annals of the New York Academy of Sciences, 1251, E124. doi: 10.1111/j.1749-6632.2012.06751.x

Phelps, E. A., \& LeDoux, J. E. (2005). Contributions of the amygdala to emotion processing: from animal models to human behavior. Neuron, 48, 175-187. doi: 10.1016/j.neuron.2005.09.025

Phillips, M. L., Drevets, W. C., Rauch, S. L., \& Lane, R. (2003). Neurobiology of emotion perception I: the neural basis of normal emotion perception. Biological Psychiatry, 54, 504-514.

Phillips, M. L., Travis, M. J., Fagiolini, A., \& Kupfer, D. J. (2008). Medication effects in neuroimaging studies of bipolar disorder. The American Journal of Psychiatry, 165, 313-320. doi: 10.1176/appi.ajp.2007.07071066

Roy, A. K., Shehzad, Z., Margulies, D. S., Kelly, A. M., Uddin, L. Q., Gotimer, K., . . Milham, M. P. (2009). Functional connectivity of the human amygdala using resting state fMRI. Neurolmage, 45, 614-626. doi: 10.1016/j. neuroimage.2008.11.030

Salvador, R., Vega, D., Pascual, J. C., Marco, J., Canales-Rodriguez, E. J., Aguilar, S., . . Pomarol-Clotet, E. (2014). Converging medial frontal resting state and diffusion-based abnormalities in borderline personality disorder. Biological Psychiatry, 79, 107-116. doi: 10.1016/j.biopsych.2014.08.026

Schulze, L., Schmahl, C., \& Niedtfeld, I. (2015). Neural correlates of disturbed emotion processing in borderline personality disorder: a multimodal meta-analysis. Biological Psychiatry, 69, 564-573. doi: 10.1016/j. biopsych.2015.03.027 
Sebastian, A., Jacob, G., Lieb, K., \& Tüscher, O. (2013). Impulsivity in borderline personality disorder: a matter of disturbed impulse control or a facet of emotional dysregulation? Current Psychiatry Reports, 15, 339.

Stiglmayr, C. E., Shapiro, D. A., Stieglitz, R. D., Limberger, M. F., \& Bohus, M. (2001). Experience of aversive tension and dissociation in female patients with borderline personality disorder -- a controlled study. Journal of Psychiatry Research, 35, 111-118.

Tambini, A., Ketz, N., \& Davachi, L. (2010). Enhanced brain correlations during rest are related to memory for recent experiences. Neuron, 65, 280-290. doi: 10.1016/j.neuron.2010.01.001

Trull, T. J., Jahng, S., Tomko, R. L., Wood, P. K., \& Sher, K. J. (2010). Revised NESARC personality disorder diagnoses: gender, prevalence, and comorbidity with substance dependence disorders. Journal of Personality Disorders, 24, 412-426. doi: 10.1521/pedi.2010.24.4.412

Uddin, L. Q. (2015). Salience processing and insular cortical function and dysfunction. Nature Reviews. Neuroscience, 16, 55-61. doi: 10.1038/nrn3857

Urry, H. L., van Reekum, C. M., Johnstone, T., Kalin, N. H., Thurow, M. E., Schaefer, H. S., . . Davidson, R. J. (2006). Amygdala and ventromedial prefrontal cortex are inversely coupled during regulation of negative affect and predict the diurnal pattern of cortisol secretion among older adults. Journal of Neuroscience, 26, 4415-4425. doi: 10.1523/JNEUROSCI.3215-05.2006

van der Werff, S. J., Pannekoek, J. N., Veer, I. M., van Tol, M. J., Aleman, A., Veltman, D. J., . . van der Wee, N. J. (2013). Resting-state functional connectivity in adults with childhood emotional maltreatment. Psychological Medicine, 43, 1825-1836. doi: 10.1017/S0033291712002942

van Zutphen, L., Siep, N., Jacob, G. A., Domes, G., Sprenger, A., Willenburg, B., . . Arntz, A. (n.d.). Always on guard: Emotion regulation in borderline personality disorder compared to non-patients and cluster-C personality disorder patients. Submitted

van Zutphen, L., Siep, N., Jacob, G. A., Goebel, R., \& Arntz, A. (2015). Emotional sensitivity, emotion regulation and impulsivity in borderline personality disorder: A critical review of fMRI studies. Neuroscience and Biobehavioral Reviews, 51C, 64-76. doi: 10.1016/j.neubiorev.2015.01.001

Veer, I. M., Oei, N. Y., Spinhoven, P., van Buchem, M. A., Elzinga, B. M., \& Rombouts, S. A. (2011). Beyond acute social stress: increased functional connectivity between amygdala and cortical midline structures. Neurolmage, 57, 1534-1541. doi: 10.1016/j.neuroimage.2011.05.074

Vermetten, E., \& Spiegel, D. (2014). Trauma and dissociation: implications for borderline personality disorder. Current Psychiatry Reports, 16, 434. doi: 10.1007/s11920-013-0434-8

Wetzelaer, P., Farrell, J., Evers, S., Jacob, G. A., Lee, C. W., Brand, O., . . Arntz, A. (2014). Design of an international multicentre RCT on group schema therapy for borderline personality disorder. BMC Psychiatry, 14, 319. doi: 10.1186/s12888-014-0319-3

Winter, D., Elzinga, B., \& Schmahl, C. (2014). Emotions and memory in borderline personality disorder. Psychopathology, 47, 71-85. doi: 10.1159/000356360

Wolf, R. C., Sambataro, F., Vasic, N., Schmid, M., Thomann, P. A., Bienentreu, S. D., \& Wolf, N. D. (2011). Aberrant connectivity of resting-state networks in borderline personality disorder. Journal of Psychiatry \& Neuroscience, 36, 402-411. doi: 10.1503/jpn.100150

Zanarini, M. C., Frankenburg, F. R., Dubo, E. D., Sickel, A. E., Trikha, A., Levin, A., \& Reynolds, V. (1998). Axis II comorbidity of borderline personality disorder. Comprehensive Psychiatry, 39, 296-302.

Zanarini, M. C., Yong, L., Frankenburg, F. R., Hennen, J., Reich, D. B., Marino, M. F., \& Vujanovic, A. A. (2002). Severity of reported childhood sexual abuse and its relationship to severity of borderline psychopathology and psychosocial impairment among borderline inpatients. The Journal of Nervous and Mental Disorders, 190, 381387. 


\section{Supplementary Materials}

The text below is almost identical to van Zutphen et al, [submitted] because it is a part of a larger study performed by the same research group.

\section{Participants}

Borderline personality disorder (BPD) and Cluster-C personality disorder (CCP) patients were recruited from the mental health clinics at local sites: the Virenze-Riagg in Maastricht (the Netherlands), PsyQ in Heerlen (the Netherlands), the borderline treatment unit of the Department of Psychiatry and Psychotherapy at the Medical Center Freiburg (Germany), the Department of Psychiatry and Psychotherapy at the University Hospital Lübeck (Germany) and the Institute for Behavior Therapy Training Hamburg (Germany). BPD patients were taking part in an international multicenter RCT on group schema therapy for BPD (for detailed description, Wetzelaer et al., 2014) and their measurements reported in this study were collected before the start of their therapy, unless impossible due to scheduling problems (scanning only) in which case the fMRI-measurements had to be finished within three months from the start of the treatment. Only females were chosen since gender might influence emotional processing (Whittle, Yucel, Yap \& Allen, 2011), and because in mental health care borderline personality disorder is more often diagnosed in females. We excluded homosexual females, because we used heterosexual erotic stimuli. General exclusion criteria were lifetime psychotic or bipolar disorder type-l, attention-deficit/hyperactivity disorder, dissociative identity disorder, serious and/or unstable medical illness, substance dependence needing clinical detoxification and fMRI exclusion criteria (i.e. claustrophobia, metal objects, cardiac arrhythmia, epilepsy, tattoos at neck/head and pregnancy).

BPD and CCP patients were diagnosed according to the DSM-IV criteria, using the Structural Clinical Interview (SCID) I (First, Spitzer, Gibbon \& Williams, 1994) and II (First, Spitzer, Gibbon, Williams \& Benjamin, 1997), assessed by trained interviewers. BPD patients were further screened with the BPD Severity Index (Arntz et al., 2003; Giesen-Bloo, Wachters, Schouten \& Arntz, 2010; Kroger et al., 2013), of which the score > 20 indicates an additional inclusion criterion for BPD (Wetzelaer et al., 2014). Narcissistic and antisocial personality disorder (PD), full or sub-threshold, were excluded for reasons related to the clinical trial in which the study sample participated (Wetzelaer et al., 2014). CCP patients were not allowed to score full or sub-threshold cluster-B PD, and > 2 BPD criteria. NPC did not meet DSMIV criteria for any Axis I or Axis II disorder, as assessed by SCID-screeners (First et al., 1994; First et al., 1997). Positive items on screeners were checked with SCID interviews. Additional assessments involved the Brief Symptom Inventory (Derogatis, 1993), BPD checklist (Arntz \& Dreessen, 1995) and Interview for Trauma Events in Childhood (Lobbestael, Arntz, HarkemaSchouten \& Bernstein, 2009). 
Eight participants were excluded because of invalid, incomplete or unavailable data (3 BPD, 2 NPC, 3 CCP), five because of excessive head movement of more than $4 \mathrm{~mm}$ (2 BPD, 2 NPC, 1 CCP), two NPC had scores above .70 on the BSI and three CCP patients had scores above 100 on the BPD checklist. Eight participants (6 BPD, 1 NPC, 1 CCP) explicitly reported in the exit questionnaire that they fell asleep during any of the resting state runs, and therefore were excluded. Three BPD and two CCP subjects were excluded because they had an IQ outside the range of 70-120. Additionally, two NPC with IQ scores above the 95-percentile were excluded to guarantee that NPC were matched to the BPD participants.

\section{Measures}

\section{Borderline Personality Disorder Severity Index (BPDSI-VI)}

The BPDSI is a semi-structured clinical interview assessing frequency and severity of borderline manifestations (Arntz et al., 2003; Giesen-Bloo et al., 2010; Kroger et al., 2013). The 70-items reflect the nine borderline criteria described in the DSM-IV. For each item the frequency of the last three months is rated on an 11-point scale, ranging from 0 (never) to 10 (daily). The scores on the subscales provide information on the severity of each of the DSM-IV dimensions, derived by averaging the items scores. The total score is the sum of the nine dimensions scores, ranging from 0 to 90, with an internal consistency of Cronbach's $a=.93$ and subscales ranged between .41 and .83 (Arntz et al., 2003). A total score of 20 distinguishes borderline personality disorder from other personality disorders (Giesen-Bloo et al., 2010).

\section{Brief Symptom Inventory (BSI)}

The Brief Symptom Inventory is a brief psychological self-report inventory of general symptoms of psychopathology during the past week (Derogatis, 1993). It is a short alternative for the Symptom Checklist-90-R from which it was developed. It contains 53 items divided over nine dimensions: somatization, obsession-compulsion, interpersonal sensitivity, depressive mood, anxiety, hostility, phobic anxiety, paranoid ideation and psychoticism. Answers are scored on a 5-point Likert scale, ranging from 0 (not at all) to 4 (extremely). Scores of the dimensions are calculated by summing the values for the items divided by the number of items within the subscales. The total score measures the level of symptomatology, which is the sum of the nine dimensions plus the four additional items divided by total number of items. The internal consistency showed a Cronbach's a of .96 for the total instrument and ranged between .71 and .85 for its subscales (De Beurs \& Zitman, 2006; Derogatis, 1993). To distinguish patients from non-patients a cutoff score of .70 is suggested (De Beurs, 2004). 


\section{BPD Checklist}

The BPD Checklist is a self-report questionnaire used to assess the burden of borderline symptoms as experienced during the last month (Arntz \& Dreessen, 1995). It consists of 47 items based on the nine dimensions of borderline personality disorder in DSM-IV. Items must be rated on 5-point Likert scale, ranging from 1 (not at all) to 5 (extremely). Next to the total score, also the scores for the nine subscales can be calculated. Scores above 100 signify borderline pathology and therefore is indicative as cutoff for inclusion criteria. When control patients showed an elevated score, an extra check with the SCID-II borderline section was done.

\section{Interview for Traumatic Events in Childhood (ITEC)}

The Interview for Traumatic Events in Childhood is a retrospective, semi-structured interview to measure childhood maltreatment prior the age of 18 including sexual ( 12 items), physical (13 items) and emotional abuse ( 9 items), and emotional (6 items) and physical neglect (15 items) (Lobbestael et al., 2009). For each item the participant experienced maltreatment, follow-up questions are used to gather more detailed information about the perpetrator(s), age of onset, frequency, duration of the trauma and the impact on the victim in the past and in the present. This information was used to calculate a severity score between 0 and 1 , such that the score increased with the severity of the event itself, the closeness of the perpetrator, the younger age of onset, the longer duration of the event, and the higher impact on the victim. For each subscales the severity scores for the events are summed, the higher this score, the more severe the maltreatment. Internal consistencies of these scales were moderate to excellent, with Cronbach's a varying between .58 and .89 with a mean of .79 (Lobbestael et al., 2009). In addition to the victimization scales, similar scales were created for witnessing the various forms of maltreatment. In current study only the victim scales were reported. On average the administration time is about 30 minutes but can take up to one hour in case of multiple maltreatments.

\section{Wechsler Adult Intelligence Scale}

IQ was estimated by means of four subtests of the Wechsler Adult Intelligence Scale, including two verbal (i.e. Vocabulary and Similarities) and two nonverbal tests (i.e. Block design and Matrix reasoning). Together these subtests correlate strongly with general intellectual ability (Schrimsher, O'Bryant, O'Jile \& Sutker, 2008). IQ was estimated based on the optimized regression equation: $39.05+\left(1.54^{*}\right.$ comprehension score $)+\left(1.64^{*}\right.$ matrix reasoning score $)+\left(1.48^{*}\right.$ similarities score $)+\left(.98^{*}\right.$ picture arrangement score). If the WAIS score was not available (borderline patients $n=11$, non-patients $n=3$ and control patients $n=1)$ an estimation of the IQ was made based on the education level, using the regression 
equation from our present sample per group; IQ borderline patients $=85.932+\left(3.360^{*}\right.$ ISCED code), IQ non-patients $=85.996+(3.659 *$ ISCED code $)$ and IQ control patients $=81.529+$ (4.226*ISCED code). These regression equations explained respectively 22\%, 31\% and 36\% of the variance in IQ. Level of education of both the Dutch and German educational systems were transformed into the International Standard Classification of Education (ISCED).

\section{Dissociation and Anxiety}

Present state dissociative experiences were assessed using four items of the DissociationTension-Scale (Stiglmayr, Shapiro, Stieglitz, Limberger \& Bohus, 2001), containing derealization and changes in perception of one's body, hearing and pain. Additionally two items concerning the level of anxiety and the level of nervousness were added. Responses were indicated on a visual analogue scale, ranging from 0 not at all to 100 extreme. The dissociation score was the averaged across the four dissociation items. Internal consistencies of the dissociation score proved to be good in current sample, with a Cronbach's $a$ of .83 concerning dissociation before scanning and a Cronbach's a of .88 concerning dissociation after scanning.

\section{Self-Assessment Manikin Scale}

The pictures shown during the scanning session were qualitatively assessed for valence and arousal using the Self-Assessment Manikin Scale. The Self-Assessment Manikin Scale consists of a series of human-like figures to measures the affective reaction of a person to stimuli (Bradley \& Lang, 1994). Intensity of valence and arousal were both rated on a 9-point scale, with for valence 1 being extremely unpleasant and 9 being extremely pleasant, and for arousal 1 being most calm and 9 being most aroused.

\section{MRI data acquisition}

Structural and functional MRI data were acquired with 3-Tesla scanners at three sites: in Maastricht on a Siemens Magnetom Allegra head-only scanner equipped with a birdcage headcoil (Siemens Medical Systems, Erlangen, Germany), in Freiburg on a Siemens tim-Trio Magnetom whole body scanner (Siemens Medical Systems, Erlangen, Germany) equipped with an 8-channel headcoil, and in Lübeck on a Philips Achiva whole body scanner equipped with an 8-channel headcoil (Philips Healthcare, Best, The Netherlands). The BPD patients from Heerlen were scanned in Maastricht and the BPD patients from Hamburg were scanned in Lübeck. In Maastricht 12 BPD, 9 NPC and 11 CCP were scanned, Freiburg scanned 13 BPD, 18 NPC and 6 CCP, finally 23 BPD, 12 NPC and 4 CCP were scanned in Lubeck. 


\section{FMRI data preprocessing}

FMRI data preprocessing was carried out using BrainVoyager 2.8 (Brain Innovation, Maastricht, The Netherlands), NeuroElf (an MR imaging analysis toolbox, www.neuroelf.net) and custom routines in Matlab (Mathworks, Inc). The first two volumes were removed due to saturation effects. The following preprocessing steps were conducted: slice time correction with sinc interpolation, 3D motion correction for three translational and three rotational directions with trilinear/sinc interpolation, spatial smoothing with a $4 \mathrm{~mm}$ full-width-at-half-maximum isotropic Gaussian kernel, and removal of linear trend. Additionally, a temporal band-pass filter of 0.01 to $0.1 \mathrm{~Hz}$ was applied to reduce cardiac and respiratory noise. In order to further reduce the non-neuronal components of fMRI datasets, a component based method (CompCor) was applied to remove the significant principal components representing data artifacts from head motion, cerebrospinal fluid, and white matter (Behzadi, Restom, Liau \& Liu, 2007).

Spatial normalization was conducted using a high-resolution anatomical datasets. Participants underwent a second fMRI session (data presented elsewhere), in which a second anatomical scan was acquired. In order to obtain a high resolution and high contrast anatomical scan, the individual anatomical datasets from the two fMRI sessions were averaged when possible. Anatomical datasets were corrected for inhomogeneity intensity and non-brain tissue was removed. Such individual anatomical datasets were used to co-register the single subject 4D functional datasets. All single subject datasets were normalized in Talairach space $(1 \times 1 \times 1 \mathrm{~mm}$; Talairach \& Tournoux, 1988). 


\section{Supplementary Figures}

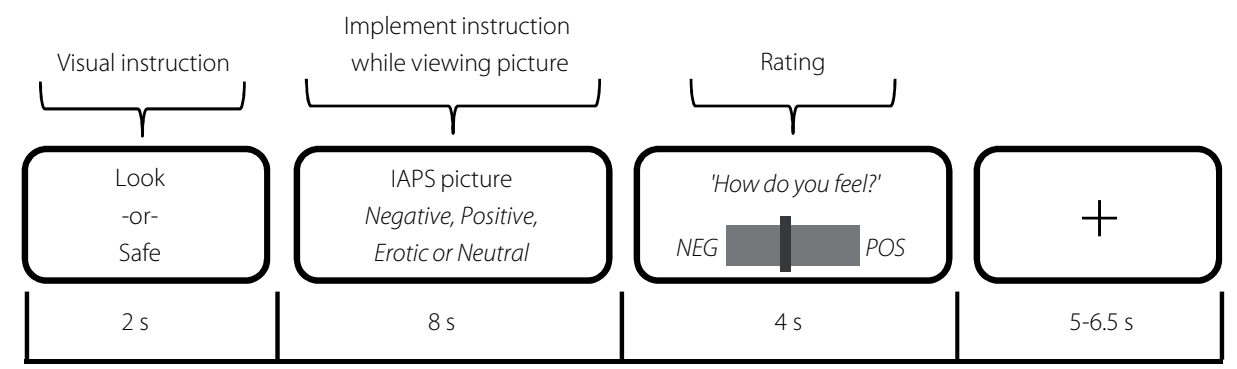

Figure S1. Schematic overview of a single trial of the Emotion regulation paradigm

Detailed description of the task is available elsewhere [van Zutphen et al., submitted], but for a schematic overview of a single trial see figure.

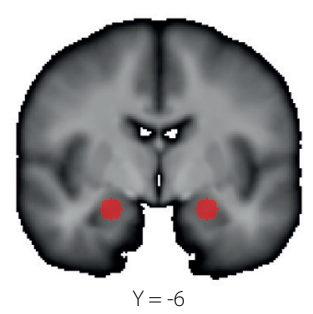

Figure S2. Amygdala seeds

A $5 \mathrm{~mm}$ sphere of the seeds used in the functional connectivity analyses were centered around the coordinates: $\pm 22,-6,-14$ (Talairach space). 


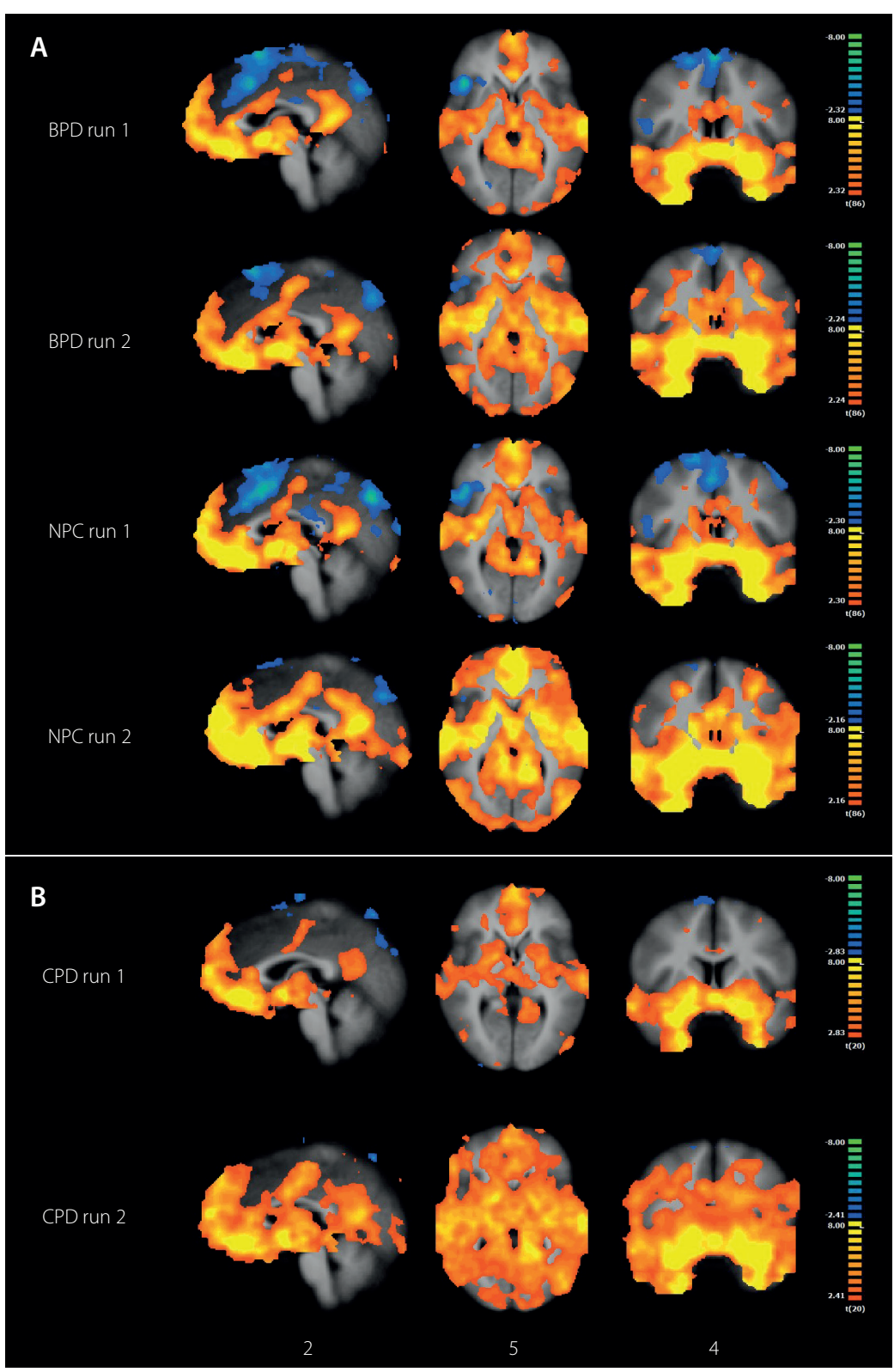

Figure S3. Main effects of the amygdala resting-state functional connectivity in BPD and NPC (Panel A), and CPD (Panel B)

The t-maps (whole brain FDR voxel-level corrected $p<.05$ ) depict average amygdala functional connectivity before and after the emotion regulation task. Statistical maps were overlaid on an anatomical image averaged over all participants in the Talairach standard space, according to the radiological convention. Positive connectivity is shown in hot colors whereas cold colors indicate negative connectivity. Significant voxels outside cerebrum were masked for illustration purposes. 


\section{References}

Arntz, A., \& Dreessen, L. (1995). BPD-Klachtenlijst 47 [BPD Checklist]. The Netherlands: Maastricht University.

Arntz, A., van den Hoorn, M., Cornelis, J., Verheul, R., van den Bosch, W. M., \& de Bie, A. J. (2003). Reliability and validity of the borderline personality disorder severity index. Journal of Personality Disorders, 17, 45-59. doi: 10.1521/ pedi.17.1.45.24053

Behzadi, Y., Restom, K., Liau, J., \& Liu, T. T. (2007). A component based noise correction method (CompCor) for BOLD and perfusion based fMRI. Neurolmage, 37, 90-101. doi: 10.1016/j.neuroimage.2007.04.042

Bradley, M. M., \& Lang, P. J. (1994). Measuring emotion: the Self-Assessment Manikin and the Semantic Differential. Journal of Behavior Therapy and Experimental Psychiatry, 25, 49-59. doi: 10.1016/0005-7916(94)90063-9

De Beurs, E. (2004). Handleiding bij de Brief Symptom Inventory (BSI). Leiden: Pits Publishers.

De Beurs, E., \& Zitman, F. G. (2006). De Brief Symptom Inventory (BSI): De betrouwbaarheid en validiteit van een handzaam alternatief voor de SCL-90 [The Brief Symptom Inventory (BSI): The reliability and validity of a brief alternative of the SCL-90]. Maandblad Geestelijke Volksgezondheid, 61, 120-141.

Derogatis, L. R. (1993). BSI Brief Symptom Inventory: Administration, Scoring, and Procedure Manual (4th ed.). Minneapolis, MN: National Computer Systems.

First, M. B., Spitzer, R. L., Gibbon, M., \& Williams, J. B. W. (1994). Structured clinical interview for DSM-IV axis I disorders (SCID-I). New York: Biometric Research Department.

First, M. B., Spitzer, R. L., Gibbon, M., Williams, J. B. W., \& Benjamin, L. (1997). Structured clinical interview for DSM-IV axis II personality disorders (SCID-II). New York: Biometric Research Department.

Giesen-Bloo, J. H., Wachters, L. M., Schouten, E., \& Arntz, A. (2010). The Borderline Personality Disorder Severity IndexIV: psychometric evaluation and dimensional structure. Personality and Individual Differences, 49, 136-141. doi: DOl 10.1016/j.paid.2010.03.023

Kroger, C., Vonau, M., Kliem, S., Roepke, S., Kosfelder, J., \& Arntz, A. (2013). Psychometric properties of the German version of the borderline personality disorder severity index--version IV. Psychopathology, 46, 396-403. doi: $10.1159 / 000345404$

Lobbestael, J., Arntz, A., Harkema-Schouten, P., \& Bernstein, D. (2009). Development and psychometric evaluation of a new assessment method for childhood maltreatment experiences: the interview for traumatic events in childhood (ITEC). Child Abuse \& Neglect, 33, 505-517. doi: 10.1016/j.chiabu.2009.03.002

Schrimsher, G. W., O'Bryant, S. E., O'Jile, J. R., \& Sutker, P. B. (2008). Comparison of tetradic WAIS-III short forms in predicting full scale IQ scores in neuropsychiatric clinic settings. Journal of Psychopathology and Behavioral Assessment, 30, 235-240. doi: DOl 10.1007/s10862-007-9066-9

Stiglmayr, C. E., Shapiro, D. A., Stieglitz, R. D., Limberger, M. F., \& Bohus, M. (2001). Experience of aversive tension and dissociation in female patients with borderline personality disorder -- a controlled study. Journal of Psychiatric Research, 35, 111-118. doi: 10.1016/S0022-3956(01)00012-7

van Zutphen, L., Siep, N., Jacob, G. A., Domes, G., Sprenger, A., Willenburg, B., . . Arntz, A. (n.d.). Always on guard: Emotion regulation in borderline personality disorder compared to non-patients and cluster-C personality disorder patients. Submitted

Wetzelaer, P., Farrell, J., Evers, S. M., Jacob, G. A., Lee, C. W., Brand, O., . . Arntz, A. (2014). Design of an international multicentre RCT on group schema therapy for borderline personality disorder. BMC Psychiatry, 14, 319. doi: 10.1186/s12888-014-0319-3

Whittle, S., Yucel, M., Yap, M. B., \& Allen, N. B. (2011). Sex differences in the neural correlates of emotion: evidence from neuroimaging. Biological Psychology, 87, 319-333. doi: 10.1016/j.biopsycho.2011.05.003 



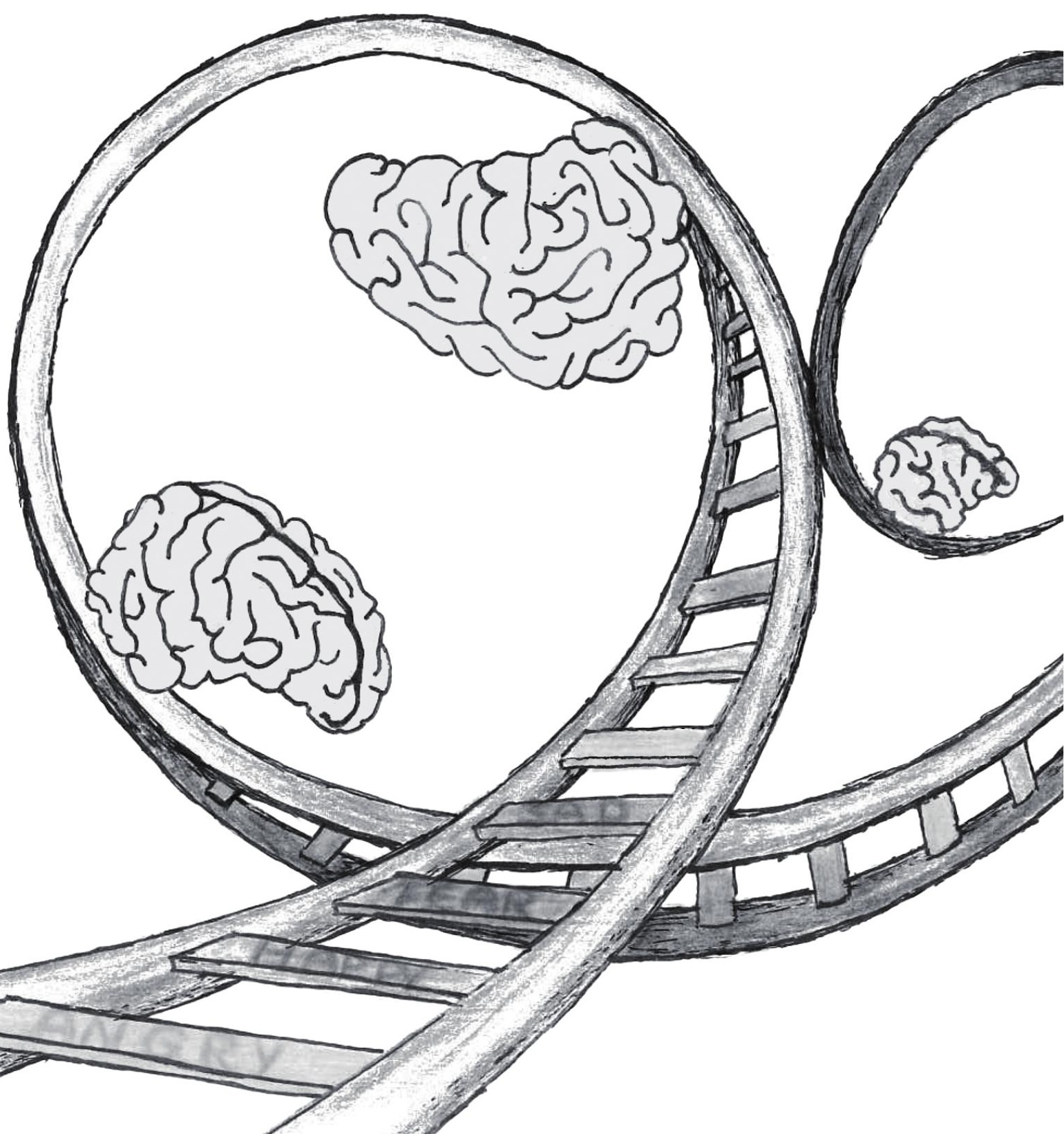




This dissertation focuses on the investigation of stimulus category specificity and diagnosis specificity of neural correlates of emotional sensitivity, emotion regulation and impulsivity in borderline personality disorder (BPD). The studies described aimed to 1) examine the generalizability across stimuli differing in their emotional content, 2) investigate the diagnosis specificity compared to other personality disorders, and 3) increase the statistical power, compared to previous studies, by increasing the sample size.

The second chapter provides a critical review of the literature on fMRI studies involving emotional sensitivity, emotion regulation and impulsivity in BPD. We built upon a couple of these findings in the subsequent chapters 3,4 and 5. More specifically, positive and erotic stimuli were added to examine stimulus category specificity, a clinical control group of Cluster-C personality disorder patients was added to examine diagnosis specificity, and the sample size was increased by recruiting participants from multiple international centers to improve the statistical power.

In this final chapter the main findings of the investigations described in this dissertation are summarized. Furthermore, reflections on theoretical, clinical as well as research implications, and recommendations for forthcoming research will be provided throughout. This chapter ends with overall concluding remarks of the current dissertation.

\section{Summary of the findings}

In Chapter 2 a critical light was shed on the methodology and results of fMRI studies examining emotional sensitivity, emotion regulation and impulsivity in BPD in response to negative stimuli. This review indicates that a higher emotional sensitivity, impaired emotion regulation and problems with impulse control in BPD can be best understood in terms of impaired inhibition from prefrontal areas on the limbic areas. However, the results across the studies appear to be more inconsistent and inconclusive as previously suggested, strong similarities but also important differences in results of various studies are reported. Evidence of an increased amygdala activity during provocation of emotional sensitivity in BPD patients was most consistent. The most consistent finding regarding (effortful) emotion regulation in BPD patients was a decreased activity of the anterior cingulate cortex, while the medial and dorsolateral prefrontal areas showed variable activity. Concerning impulsivity decreased activity of inhibitory areas in BPD patients was inconsistent. For replications of the results of previous studies and answering unanswered issues such as generalizability and specificity, future research should take into account the limited power of previous studies and increase the sample sizes. Additionally, future research should focus on BPD specificity with respect to (delayed) habituation effects of amygdala, different emotion regulation strategies, manipulation of self-regulation, use of psychotropic medication, and gender differences. Lastly, extending the range of stimuli (e.g. positive and non-social) and the importance of the inclusion of a clinical control group were discussed. A better understanding of the 
underlying neural correlates of emotional sensitivity, emotion regulation and impulsivity open new avenues to future research and provide valuable insights to refine and improve therapies.

To improve our understanding of BPD the next chapters 3, 4, and 5 tap into a couple of the issues pointed out in the critical review of chapter 2. The studies of current dissertation extended previous research by a wider range of stimulus categories with positive stimuli, and given the high rates of childhood sexual abuse and difficulties with intimacy in BPD erotic stimuli were added. Furthermore, a clinical control group of patients with Cluster-C personality disorder was added. Finally, participants were recruited in multiple international centers to increase the sample size. Chapter $\mathbf{3}$ focuses on stimulus category specificity and diagnosis specificity of emotional sensitivity and emotion regulation in BPD. During fMRI scanning, an adapted version of a classic emotion regulation paradigm was presented, which required the participants to alternate between the passive viewing of social emotional scenes and the effortful regulation of the emotions elicited by these emotional scenes. As regulation strategy participants were instructed to realize they were safe, inspired by schema therapy theory. Results showed that passive viewing of negative stimuli resulted in higher activity in the anterior insula in BPD patients compared to non-patients. However, we could not replicate previous findings of higher amygdala responses in BPD. Additional less stringent amygdala region-of-interest analysis could not detect significant interaction effects, even not at $p<.05$ uncorrected. Other potential confounding factors including dissociation, trauma, posttraumatic stress disorder, depression, medication, lateralization or fast habituation could also not explain this null finding. Interestingly contrary to previous studies, passive viewing of negative stimuli also led to higher activity in the temporoparietal junction and dorsolateral prefrontal cortex in BPD. During regulation of negative stimuli, non-patients showed higher activity in the dorsal anterior cingulate cortex, dorsolateral prefrontal cortex, middle temporal gyrus and bilateral inferior parietal lobule compared to passive viewing. BPD patients did not show this increase in activity when instructed to realize to be safe compared to the look condition. In conclusion, when passively viewing emotional social stimuli BPD patients showed an increased response in brain areas important for generation as well as regulation of emotions, suggesting an unique pattern of an increase brain activity involved in mentalizing processes (resonate with mental states of others; i.e. temporoparietal junction) and emotional bodily states (i.e. anterior insula). In the case of negative stimuli this is accompanied by increased activity in regulation areas (i.e. dorsolateral prefrontal cortex). In contrast, increase of regulation areas seems absent when BPD patients are instructed to do so. Additionally, BPD patients showed an inability to activate attentional areas (i.e. inferior parietal lobule) during emotion regulation which might interfere with cognitive control. As the increased response in the emotion generation areas was also present during passive viewing of positive stimuli, results regarding emotional sensitivity 
were not specific for negative stimuli, whereas results regarding emotion regulation were specific for negative stimuli. Diagnosis specificity by means of linearity analyses supports a dimensional rather than a dichotomous differentiation of Cluster- $C$ patients in-between BPD and non-patients.

In Chapter $\mathbf{4}$ we investigated stimulus category specificity and diagnosis specificity of impulsivity in BPD. While measuring brain activity participants performed an affective go/ no-go task with different categories of emotional social stimuli. Behavioral data showed that BPD patients made more omission errors than non-patients, indicating deficits for attentional processing or a general inhibition to prevent commission errors, while motor inhibition itself was preserved as indicated by comparable commission errors. Stimulus category had no effect on the number of omissions. Neuroimaging results showed higher activity in the frontal eye fields when inhibiting negative stimuli in BPD, while non-patients showed less activity in the inferior parietal lobule when inhibiting negative stimuli. These findings did not replicate the hypothesized altered prefrontal activity in BPD as reported in previous studies. In both clusters the comparison BPD versus Cluster-C patients revealed no differences, indicating that activity in these clusters is not BPD specific. Diagnosis specificity by means of linearity analyses supports a dimensional rather than a dichotomous differentiation of intermediate responses of Cluster- $C$ patients placed between BPD and non-patients. Results concerning stimulus category specificity showed that the inferior parietal lobe also showed a significant interaction involving positive and erotic stimuli, caused by an activity modulation across stimulus categories in non-patients. This modulation by positive and erotic stimuli was absent in BPD, indicating they are not affected by the stimulus category. The frontal eye fields did show higher activity during the presentation of the negative stimuli in BPD compared to the other stimuli categories, this effect was not present in the non-patients or control patients. As both areas are assigned to the left dorsal attention network, involved in top-down attentional control over brain areas of the sensory cortices and ventral attention network, we speculate that the findings hint towards an impaired top-down attentional bias implementation and inappropriate top-down control over the ventral attention network in BPD. Supported by the behavioral results, BPD patients showed no deficiencies in emotionally modulated response inhibition per se but rather hint to early stage attentional difficulties for emotional information.

Finally in Chapter 5, resting-state fMRI was used to investigate enduring effects of effortful emotion regulation on the amygdala intrinsic functional connectivity in BPD as emotion dysregulation in BPD has been associated with an impaired amygdala-prefrontal inhibitory network. Resting-state fMRI was acquired before and after the emotion regulation task. Although behavioral results of experienced emotion (visual analogue scale negative to positive) immediately after the passive viewing and regulation condition showed a similar change in BPD patients' and non-patients' emotional state due to cognitive regulation, BPD 
patients exhibited a different resting-state functional connectivity after the task. In contrast to non-patients, BPD patients failed to show post-task increased resting-state functional connectivity between the amygdala and brain areas important for effortful emotion regulation including the medial, dorsolateral and ventrolateral prefrontal cortex and superior temporal gyrus. Surprisingly, BPD patients showed a post-task decreased amygdala resting-state functional connectivity with the posterior cingulate cortex involved in selfreferential processing and autobiographic memory, and increased amygdala resting-state functional connectivity with the superior parietal lobule involved in attention and episodic memory retrieval. A comparison with the Cluster- $C$ group suggested that the direct regulation of the amygdala via the ventromedial prefrontal cortex is not specific for BPD, but seems to be common for personality disorders. Furthermore, a positive correlation was found between the BPD Severity Index and the post-task changes of the intrinsic amygdala connectivity in the ventrolateral prefrontal cortex. We suggest that severely affected BPD patients engaged more the ventrolateral prefrontal cortex to maintain their reappraisals in working memory. To overcome this deficit, the positive association between increase in the level of dissociation and the inferior frontal gyrus and the superior temporal gyrus suggest a tendency for a compensatory but maladaptive strategy, such as dissociation. In addition, a negative correlation was found between the Interview of Traumatic Events in Childhood and the post-task changes of the intrinsic amygdala connectivity in the insula. We suggest that BPD patients tend to avoid self-related appraisals induced by social emotional stimuli, which might be partly mediated by their blunted affect caused by childhood maltreatment.

\section{General discussion}

The purpose of the current dissertation was to investigate BPD uniqueness, with a focus on stimulus category specificity and diagnosis specificity, of the neural correlates of emotional sensitivity, emotion regulation (Chapter 3) and impulsivity (Chapter 4) in BPD. Extending previous research, we added positive and erotic stimuli to the traditional negative and neutral stimuli, and compared the BPD group to a non-patient and Cluster-C personality disorder group. In line with our hypothesis of higher emotional sensitivity in BPD, passive viewing of negative stimuli resulted in elevated responses in the anterior insula - important for emotion generation - in BPD compared to non-patients. However, we could not replicate previous findings of higher amygdala responses to negative emotional stimuli in BPD. Passive viewing of negative stimuli also led to higher activity in the temporoparietal junction and dorsolateral prefrontal cortex in BPD. Furthermore, consistent with our hypothesis of impaired emotion regulation abilities in BPD, BPD patients did not engage in increased activity in the dorsolateral prefrontal cortex and dorsal anterior cingulate cortex - brain areas important for emotion regulation - during the safe (i.e. regulation) versus look condition when presented with negative stimuli, whereas non-patients did show this 
increase in activity during the safe versus look condition. In contrast to our expectations, we did not found support for the hypothesized impaired response inhibition in BPD. We could not replicate the altered prefrontal activity reported in previous studies on BPD impulsivity, instead we observed altered responses in the inferior parietal lobule and frontal eye fields in BPD patients when inhibiting social emotional stimuli. Finally, we examined enduring effects of an emotion regulation task on the amygdala intrinsic functional connectivity in BPD (Chapter 5), more specifically, whether this induces different changes of amygdala restingstate functional connectivity with the prefrontal cortex in BPD patients compared to nonpatients. Consistent with our hypothesis BPD patients failed to increase amygdala intrinsic functional connectivity with the medial, dorsolateral and ventrolateral prefrontal cortices, essential for emotion regulation, which might hinder cognitive control of emotions in BPD. In sum, BPD patients show on the one hand hyperactive bottom-up emotional sensitivity, and on the other hand impaired top-down control in emotion regulation and impulse control. Additionally, considering the passive viewing condition of the emotion regulation task and the go/no-go task as implicit forms of emotion regulation also evidence is shown for indications of deficits (already) at the level of implicit emotion regulation for negative emotions in BPD patients.

A part from the fMRI studies to localize structures underlying altered emotional sensitivity, emotion regulation and impulsivity in BPD, studies explicitly addressing functional interactions are necessary to better understand these neural mechanisms. Future research should follow up by functional connectivity analyses on the generated hypothesis concerning a dysfunctional top-down processing during emotion regulation and impulse control in BPD, and examine more closely whether this fronto-limbic network either directly or indirectly contributes to emotional processing problems in BPD. Increased fronto-limbic coupling in BPD patients has been reported between the amygdala and subgenual anterior cingulate cortex when viewing fearful faces (Cullen et al., 2011), and with the dorsomedial prefrontal cortex during an emotional working memory task (Krause-Utz et al., 2014a). Taskfree resting-state showed that emotional hypersensitivity of BPD patients is associated with increased connectivity between the amygdala and the salience network (i.e. insula and dorsal anterior cingulate cortex) (Cullen et al., 2011; Krause-Utz et al., 2014b; Wolf et al., 2011), while their impaired control over emotional reactions is associated mostly with decreased connectivity within and between the central executive fronto-parietal areas and the salience network at rest (Doll et al., 2013; Wolf et al., 2011). Taken together, BPD patients show altered functional connectivity when confronted with negative stimuli and at rest. However, functional interaction during emotion regulation and impulse control has yet to be examined. Using effective connectivity would it even make possible to estimate the directed influence between brain areas, because it takes temporal dynamics into account. 


\section{Indications of attentional processing difficulties}

When BPD patients are confronted with social-emotional triggers, it is suggested they have difficulties regulating their emotions or inhibiting their impulsive responses due to failure to activate regulatory and inhibitory areas (e.g. ventrolateral and dorsolateral prefrontal cortices and dorsal anterior cingulate cortex). However, in Chapter 3, $\mathbf{4}$ and $\mathbf{5}$ indications also point to possible difficulties in attentional processing in BPD. The inability of BPD patients to activate the inferior parietal lobule during emotion regulation (Chapter 3 ) and altered activity of the left dorsal attention network (i.e. frontal eye fields and inferior parietal lobule) during response inhibition (Chapter 4) might interfere with top-down cognitive control. Previous research suggests that the inferior parietal lobule within the attentional network is involved in the detection of relevant stimuli and preventing reorientation of attention to unimportant stimuli, and the frontal eye fields modulate top-down control to direct attention to important stimuli (Corbetta, Patel \& Shulman, 2008). Furthermore the increased amygdala resting-state functional connectivity with the superior parietal lobule after emotion regulation (Chapter 5), might interfere with the ability to increase the functional connectivity with brain areas important for emotion regulation as top-down control is interfered with sustained attentional focus to irrelevant stimuli. Although it should be acknowledged that this hypothesis warrants further research using attention paradigms there is a theoretical base for this presumption.

Studies show that emotional information captures more attention in BPD patients compared to non-patients (Arntz, Appels \& Sieswerda, 2000; Kaiser, Jacob, Domes \& Arntz, 2016; von Ceumern-Lindenstjerna et al., 2010), even when this information is irrelevant to the task. In other words, BPD patients may have difficulties to disengage from threatrelated stimuli, which subsequently induce arousal and distress, resulting in less efficient inhibition of this attentional bias towards these negative stimuli. It has been suggested that the ability to control attention facilitates emotion regulation, but whether attentional bias in BPD plays a causal role in emotion regulation is unclear. The model of emotion regulation distinguishes five emotion regulation approaches specified as a sequence of processes involved in emotion generation: situation selection, situation modification, attentional deployment, cognitive change, and response selection (Gross, 2007). Attention is a bottleneck in many information processing streams, therefore at the early processing phase the demands of constant shifting attention towards irrelevant stimuli and/or difficulties withdrawing their attention from emotional stimuli are high for BPD patients and might exhaust cognitive resources required to allocate attention from salient stimuli and interfere with higher cognitive processes. An fMRI study in non-patients revealed after effortful selfregulation a failure of top-down control of the amygdala, indicated by weaker amygdalaventromedial prefrontal cortex connectivity (Wagner \& Heatherton, 2013). Moreover, biased attentional processing might be mood-dependent which can develop into a vicious circle. 
When in a negative mood, BPD patients might be limited to control their attention and have more difficulty disengaging from negative stimuli what might intensify the negative mood. A recent fMRI study suggested that the top-down/bottom-up model is insufficient to explain emotion dysregulation in BPD, as negative affective interference with cognitive processing is due to stimulus- or attention-driven demands of the task (Soloff, White, Omari, Ramaseshan \& Diwadkar, 2015). Attention-driven tasks (Go/No-go and X-CPT) resulted in hyperactivity in brain areas involved in response inhibition, attention and conflict resolution and episodic memory encoding and memory retrieval (orbitofrontal cortex, anterior cingulate cortex and hippocampus), whereas hypoactivity was observed during the stimulus-driven episodic memory task in BPD patients compared to non-patients (Soloff et al., 2015). Training that use techniques to modify or control attentional processes could be useful for BPD patients. However, studies to teach BPD patients to disengage attention from negative stimuli and implement avoidant attentional styles have not been conducted yet. According to the cognitive theory of personality disorders, BPD patients see others as dangerous and malevolent, and themselves as powerless, vulnerable and unacceptable (Arntz, Dreessen, Schouten \& Weertman, 2004; Pretzer, 1990). In such a world and having a negative self-image it is understandable and functional to develop an attentional bias aimed at rapid detection of signals of potential threat. On the one hand, an attentional bias is an important factor in maintaining the disorder, therefore attentional bias modification training targets coping mechanisms by expanding horizons and aid processing of the information incongruent to the attentional bias. On the other hand, it is suggested that an attentional bias is the results of underlying maladaptive schemas, in that case such a training will not be sufficient to change these schemas, which is done in schema therapy. Taken together, attentional bias modification training might be questionable in isolation, however, could be additive to cognitive treatments.

To our knowledge here-to-date no research is done concerning the neural mechanisms of attentional bias in BPD. Future studies could examine whether the visual cortex (or fusiform face area) is modulated by the amygdala in BPD patients, contributing to hyperawareness of emotional stimuli, or whether impaired discrimination or perception are the underlying mechanisms of the disturbed attentional processing in BPD. An fMRI study investigating anticipation of emotional stimuli in BPD patients compared to non-patients, presented higher activity during the anticipation of negative stimuli in the pregenual anterior cingulate, posterior cingulate and visual cortices, reflecting increased emotional sensitivity (Scherpiet et al., 2014). Additionally, during the anticipation of negative as well as potentially negative (ambiguous) stimuli BPD patients exhibited less activity in emotion regulation areas (i.e. dorsal anterior cingulate cortex, midcingulate cortex and dorsolateral prefrontal cortex) representing emotion dysregulation and poor impulse control associated with BPD. These results demonstrate a disturbed emotion processing biased towards negative stimuli and 
diminished emotion regulation already during emotional anticipation. It is also possible that the heightened sensitivity to detect and perceive emotional stimuli in BPD patients might be a preconscious automatic process, even prior to explicit presentation of the emotional stimuli. Two recent fMRI studies investigated preattentive emotional processing in BPD by means of masking paradigms (Baskin-Sommers et al., 2015; Cullen et al., 2015). The first study found in response to covert fearful faces (masked) greater activity in attentional and regulatory areas in BPD patients compared to non-patients, reflecting an overactive system already during preattentive emotion processing (Cullen et al., 2015). The second study also showed enhanced activity in the amygdala, midcingulate cortex and prefrontal cortex to happy, angry and fearful versus neutral faces presented below the level of conscious awareness in BPD patients compared to non-patients (Baskin-Sommers et al., 2015).

Taken together, an attentional bias towards negative stimuli might interfere with cognitive control such as emotion regulation and impulse control. Continuous reorientation and/or slowed disengagement might exhaust cognitive resources required to disengage attention from irrelevant stimuli. Attentional and regulatory areas are in BPD patients already activated during the anticipatory and preattentive phase of emotional processing. Attentional biased modification training might be useful for BPD patients.

\section{Stimulus specificity: Social stimuli}

The results of Chapter $\mathbf{3}$ with regard to stimulus category specificity indicate a general reactivity to emotional sensitivity during the bottom-up processes, whereas the top-down processes such as emotion regulation are unique for negative stimuli. In Chapter 4 BPD patients showed a general responsivity across stimulus categories in the inferior parietal lobe, whereas effects in the frontal eye fields were specific for negative stimuli. Certain questions however remain open, as only social stimuli were used in the studies of the current dissertation. BPD is characterized by difficulties in social information processing, therefore social stimuli might have elicited increased brain activity in for instance the anterior insula, temporoparietal junction and inferior parietal lobule in BPD patients. To examine this, comparisons between social emotional versus non-social emotional (e.g. disgust) stimuli, or neutral social versus neutral non-social stimuli are needed. One of the most important factors underlying emotional and social functioning is the ability to recognize emotions. Research concerning the perception of emotions in BPD has been mixed, there are studies showing decreased facial recognition accuracy in BPD compared to non-patients, while other studies showed more accurate facial recognition (see for reviews Daros, Zakzanis \& Ruocco, 2013; Mitchell, Dickens \& Picchioni, 2014). A question of interest would be whether BPD patients are maybe more reactive to certain types of social stimuli, e.g. social scenes versus facial expressions. One could argue that facial expressions are a primary source of social information compared to complex social scenes. Studies reported emotional reactivity 
in the amygdala to neutral faces (Donegan et al., 2003) as well as to neutral social scenes (Niedtfeld et al., 2012; Schulze et al., 2011) in BPD. However, no studies to date have directly compared differences in brain activity during neutral faces versus neutral social scenes in BPD. This might be of additional interest since experimental studies (Domes, Schulze \& Herpertz, 2009; Wagner \& Linehan, 1999) revealed that BPD patients tend to interpret neutral faces as more negative compared to non-patients, and possibly perceive them not as actual neutral. Similar levels of amygdala activity across neutral and emotional stimuli, also raises the question whether BPD patients are impaired in discriminating between various emotional stimuli. Future work might advocate investigating the types of stimuli that do and do not elicit altered levels of brain activity (e.g. amygdala, anterior insula) in BPD patients.

In addition, it is useful to examine other BPD relevant stimuli. Two studies investigated the neural correlates related to interpersonal relationships in BPD; attachment trauma (Buchheim et al., 2008) and social exclusion (Domsalla et al., 2014). The first study found in BPD patients compared to non-patients, more activity in the dorsal anterior midcingulate cortex while telling stories in response to pictures including characters facing attachment threats alone (monadic), and more activity in the superior temporal sulcus and less activity in the parahippocampal gyrus during telling stories in response to pictures containing an interaction between characters in an attachment context (dyadic) (Buchheim et al., 2008). The authors interpret these results as a fear-based intolerance of aloneness, hypersensitivity to the social environment and reduced conceptualization of positive relationship memories. The second study used a cyberball task (involves a group of three people tossing a ball back and forth), BPD patients showed less activity in the dorsomedial prefrontal cortex during the inclusion condition, and higher activity in the dorsolateral prefrontal cortex during the exclusion condition compared to non-patients (Domsalla et al., 2014). The most remarkable results were observed during the control condition (i.e. predefined rules to toss only to the right or left), in which BPD patients showed higher activity in brain areas related to social exclusion namely the dorsolateral and dorsomedial prefrontal cortex, precuneus and insula compared to non-patients. These results indicate that BPD patients have difficulties with discriminating between different forms of social interactions and tend to hypermentalize during social interactions that are not determined by intentions of social partners. Another explanation could be that BPD patients have difficulty trusting the actions and motives of others (King-Casas et al., 2008). Taken together, both studies reported evidence for neural mechanisms underlying interpersonal problems in BPD. Given that traumatic events (e.g. sexual, physical and emotional abuse or neglect) and interpersonal problems (e.g. experiences of abandonment, social rejection and insecure attachment) are common in BPD (Lazarus, Cheavens, Festa \& Rosenthal, 2014; Lobbestael, Arntz \& Bernstein, 2010) neural correlates of these kind of BPD salient stimuli warrant more attention to examine whether and to what extent they trigger intense emotional reactions in BPD patients. 


\section{Diagnosis specificity: Dimensional approach}

In Chapter $\mathbf{3}$ and $\mathbf{4}$ support is found for a linear trend of Cluster- $C$ personality disorder patients responses in-between non-patients and BPD patients. This supports the idea that Cluster- $C$ personality disorder patients exhibit a number of features, yet weaker, in common with BPD. Considering that social stimuli were used, it makes sense that Cluster-C patients show similar response patterns as BPD patients, as both personality disorders show interpersonal difficulties. Consequently, it might be useful to manipulate social versus nonsocial stimuli, to examine whether this dimensionality holds. Furthermore, in Chapter $\mathbf{5}$ it is speculated that the direct regulation of the amygdala by the ventromedial prefrontal cortex is not specific for BPD but generalizes over personality disorders with common social emotional difficulties. Together, these results are in favor of dimensional rather than a categorical differentiation, which is interesting with respect to the debate concerning the categorical versus dimensional approach of mental disorders.

The Diagnostic and Statistical Manual of Mental Disorders uses categorical approach to diagnose disorders; based on the presence or absence of several symptomatic criteria one receives the diagnosis or not (American Psychiatric Association, 2013). For a diagnosis of BPD at least five out of nine criteria must be met. Critics of the categorical approach point to excessive overlap or diagnostic comorbidity, arbitrary thresholds for diagnoses, loss of potentially important clinical information, unclear boundaries with normal personality, no accurate measure for change over time, heterogeneity within same diagnosis and high percentages of personality disorders not otherwise specified (Widiger \& Trull, 2007). Therefore it has been suggested that a dimensional approach, which views personality traits on a continuum, might be more suitable. In line with the dimensional approach of personality disorders, taxometric and factor analytic studies have suggested that BPD has an underlying dimensional construct (Arntz et al., 2009; Giesen-Bloo, Wachters, Schouten \& Arntz, 2010; Sanislow et al., 2002; Trull, Distel \& Carpenter, 2011).

In addition, BPD has much co-occurrence with a variety of psychiatric disorders. So to rule out effects contributed by the comorbid disorders and examine the diagnosis specificity additional clinical control groups need to be included. The two most common comorbidities of Axis I are major depressive disorder (61-83\%) and posttraumatic stress disorder (36-56\%) (McGlashan et al., 2000; Zanarini et al., 1998a; Zimmerman \& Mattia, 1999). Comparing the neural correlates of traumatic memory in BPD patients with and without posttraumatic stress disorder demonstrated in the subgroup without posttraumatic stress disorder activity in the orbitofrontal cortex, whereas the subgroup with posttraumatic stress disorder exhibited activity in temporal (including the amygdala), posterior cingulate and occipital cortices (Driessen et al., 2004). These results indicated that absence or presence of posttraumatic stress disorder in BPD patients involve different neural correlates during recall of traumatic memories. Given that traumatic experiences are very common in 
BPD patients, it has to be clarified whether these findings stem from traumatic events or relate to comorbid posttraumatic stress disorder. Another study found a lower activity in the dorsolateral prefrontal cortex and higher activity in the anterior cingulate cortex and hippocampus in the mood disorder patients with BPD compared to non-patients during emotional face processing (Radaelli et al., 2012). Mood disorder patients without BPD showed an intermediate pattern, indicating an interaction between BPD and mood disorders. BPD, mood disorders and posttraumatic stress disorder may be conceptualized as a spectrum of chronic stress-related disorders. From Axis II BPD patients have the most co-occurrence with Cluster-C personality disorders (McGlashan et al., 2000; Zanarini et al., 1998b). In the current dissertation we included a clinical control group of Cluster-C personality disorder patients. No significant difference with regard to emotional sensitivity, emotion regulation (Chapter 3) and impulse control (Chapter 4) of social emotional stimuli was found for the comparison of BPD patients versus Cluster-C personality disorders patients, indicating that these results were not diagnosis specific for BPD.

Recently the study of basic functional domains (i.e. negative valence, positive valence, cognitive, social, and arousal/regulatory systems) that play a role in various forms of psychopathology has been promoted as a better way to gain understanding of mental disorders, than the study of categorical disorders (Cuthbert, 2014; National Institute of Mental Health, 2008). The goal of the so called Research Domain Criteria project is to develop new ways of classifying mental disorders based on genetics, neuroscience and behavioral science, to provide a foundation for precision diagnostics and treatment. Research could focus on emotion regulation problems and impulsivity in general, rather than investigate single disorders. One problem is that it is difficult to determine what the essential underlying processes are. Emotion regulation and impulsivity, for instance, in itself are both broad concepts, and it is challenging to develop a definition relevant for different kinds of psychopathology. Although high on the agenda, some concerns regarding a dimensional model should also be acknowledged. These are, difficulties reaching consensus about the structure (18 alternative proposals, e.g. five factor model), complexity and potential difficulties in implementation since there are no qualitative distinctions, and clinical utility including targets for treatment (Widiger \& Trull, 2007). At the moment it seems difficult to tell whether the dimensional approach will bring the field further, thought there is certainly an appeal in it.

\section{Notes on the amygdala}

When the emotion dysregulation theory of BPD was first proposed (Linehan, 1993) the main focus was on hyperreactivity of the amygdala. In contrast to previous studies (Herpertz et al., 2001; Koenigsberg et al., 2009b; Schulze et al., 2011) we were not able to replicate the increase of amygdala activity in BPD during passive viewing of emotional stimuli (Chapter 3). Besides 
the high sensitivity and intense reactivity to emotional stimuli, it would be worthwhile to take into account the time course of the amygdala, especially since the theory of Linehan makes a statement about delayed habituation effect regarding emotional responses in BPD. It is suggested that BPD patients are characterized by prolonged emotional experience due to a slow return to baseline arousal compared to non-patients. Research in non-patients shows that the amygdala response rapidly declines when repeatedly presenting facial expressions (Breiter et al., 1996) and emotional visual scenes (Fischer, Furmark, Wik \& Fredrikson, 2000). Several fMRI studies showed evidence for a prolonged amygdala response after repeatedly presented pictures in BPD (Hazlett et al., 2012; Kamphausen et al., 2013; Koenigsberg et al., 2014). Two recent studies suggest that this delayed habituation is BPD-diagnosis specific and not generalizes over personality disorders, as the neural features were distinct from patients with schizotypal personality disorder (Hazlett et al., 2012) and avoidant personality disorder (Koenigsberg et al., 2014). Therefore, accounting for habituation could give us better insight in emotional processing of BPD patients. To examine whether habituation could explain our null findings regarding the differential amygdala activity, we did run an additional timing analysis, taking the fast habituation of amygdala responses into account during passive viewing of negative stimuli (Chapter 3). No group difference between BPD patients and non-patients was revealed during either the first run of the emotion regulation paradigm or throughout the experiment (run 1 to 4). These results indicated that prolonged emotional experience or delayed habituation could not explain this null finding.

Another issue that could influence the activity of the amygdala in BPD is dissociation. Dissociation involves a sense that the selfor the world is unreal by a detachment from physical or emotional experiences including fragmentation of consciousness, memory, identity, perception, body representation, motor control and behavior. Dissociative symptoms are common in BPD, possibly triggered by stress or traumatic intrusions (American Psychiatric Association, 2013; Korzekwa, Dell, Links, Thabane \& Fougere, 2009; Zanarini, Ruser, Frankenburg \& Hennen, 2000). According to the fronto-limbic disconnection model of dissociation, dissociative symptoms could account for under-arousal (Sierra \& Berrios, 1998), in which the medial prefrontal cortex inhibits the amygdala and dampens the emotional reactivity and the autonomic response. Experimental studies indicate a moderating factor of dissociation in emotional learning (Ebner-Priemer et al., 2009), and psychophysiological responses during processing of emotional stimuli (Barnow et al., 2012; Ebner-Priemer et al., 2005). Furthermore, an fMRI study (Winter et al., 2015) showed evidence for inefficient cognitive inhibition of negative stimuli during an emotional Stroop task after dissociation induction in BPD patients by an increased activity in the inferior frontal gyrus and dorsolateral prefrontal cortex, both involved in inhibitory control processes, compared to BPD patients without dissociation induction. Together, individual differences of dissociation should be taken into account as BPD patients with high state dissociation might have difficulty to 
adequately process (emotional) information. Based on this evidence we performed an additional analysis controlling for dissociation during passive viewing of negative stimuli (Chapter 3) which could not explain the absence of differential amygdala activity between BPD patients and non-patients.

In sum, although checking for potential confounding factors such as habituation of the amygdala and dissociation no evidence was observed in favor of the important role of the amygdala in the emotion dysregulation hypothesis. The amygdala is sensitive for salience, novelty and arousal of relevant stimuli and plays a critical role in emotion generation, perception and encoding. However, when differentiating BPD patients from non-patients, our findings rather hint at a key role of the anterior insula and temporoparietal junction in BPD concerning emotional sensitivity (Chapter 3). Previous research suggests that the anterior insula is involved in subjective awareness of one's emotional state and affective experience (Craig, 2009), and the temporoparietal junction is proposed to be involved in mentalizing abilities to emphasize with other's emotions (Frith \& Frith, 2006; Shamay-Tsoory, 2011). These findings might suggest that maybe BPD patients engage in mentalizing processes when confronted with social emotional stimuli, which affect their own emotional bodily state (interoceptive awareness) and affective experience. Therefore, instead of the assumption that BPD is associated by emotional hyperreactivity of the amygdala and therefore have difficulty to detect and encode emotional stimuli, our findings suggest that indicated by the higher activity of the anterior insula and temporoparietal junction BPD patients constantly empathize with other's emotions and project these emotions to their own emotional state (e.g. arousal) which results in the characteristic intense emotional experience in BPD. This idea is in line with an fMRI study investigating cognitive and affective empathy in BPD (Dziobek et al., 2011), reporting less activity in the posterior superior temporal gyrus/sulcus during cognitive empathy (i.e. capacity to infer other's mental state) and increased activity in the mid-insula and anterior superior temporal sulcus during affective empathy (i.e. inappropriate reaction to another person) in BPD patients compared to non-patients. As the superior temporal sulcus mediates thinking about others (Saxe, Xiao, Kovacs, Perrett \& Kanwisher, 2004) and the insula indicates increased arousal, this supports the notion that BPD patients misinterpret other's emotions when emotionally aroused. Taken together, being aroused might result in false interpretation of mental states and interfere with the processing of the actual emotional content.

\section{Realize being Safe}

In Chapter $\mathbf{3}$ we used an adapted version of a classic emotion regulation paradigm, in which the regulation strategy, the so called Safe condition, required participants to realize they were safe. This regulation strategy was based on one of the components of schema therapy that 'unmet safety needs during childhood' underlie emotional problems (Arntz \& 
van Genderen, 2009). Accordingly, it is expected that BPD patients would have difficulty feeling safe while experiencing negative emotions as they are intrinsically threatening for them even when realizing they are in a currently safe situation. This strategy might best be understood as a reappraisal strategy, compared to distraction. During regulation (vs. look) of negative stimuli our results showed higher activity in the dorsal anterior cingulate and dorsolateral prefrontal cortices in non-patients, while BPD patients did not show this increase in activity. These findings suggest that increase of regulation processes seems absent when BPD patients are explicitly instructed to realize being safe. The specific failure of BPD patients to activate emotion regulation areas when instructed to realize they are safe is in line with the view that BPD is characterized by a lack of safety when experiencing negative emotions, even when reminded, and will maintain their cognitive schemas of an unsafe and threatening world (Arntz \& van Genderen, 2009). An alternative explanation why in BPD patients this increase in activity during explicit emotion regulation seems absent might be that BPD patients already regulate during passive viewing. This is supported by our observation in BPD patients of increased activity in the dorsolateral prefrontal cortex in the Look condition, and this activity remained nearly constant in the Safe condition. As a result BPD patients might always be on guards for signs of rejection, abuse or abandonment. An important goal of treatment might be to help patients experience negative emotions whilst feeling safe, so that negative emotions no longer need to be avoided and can be processed. If BPD patients would be able to use the Safe instruction to regulate negative emotions after treatment, it is hypothesized that they will be able to activate emotion regulation areas and modulate emotion generation areas. A forthcoming study from our lab investigates whether the ability of BPD patients to profit from realizing the present situation is safe improves along treatment.

\section{Methodological considerations: Strengths and limitations}

The strengths of the current dissertation include adding positive and erotic stimuli to the traditional negative and neutral stimuli, the inclusion of the clinical control Cluster-C personality disorder group, and increasing the sample size to increase the statistical power. However, some limitations need also to be recognized.

\section{Medication}

About $75 \%$ of the BPD patients are regularly taking psychotropic medication (Lieb, Zanarini, Schmahl, Linehan \& Bohus, 2004), we decided not to exclude patients on medication to recruit a representative and severe clinical sample. However, medication might affect brain responses and is therefore a potential confounding factor (Delaveau et al., 2011; $\mathrm{Ma}, 2015)$. As adding medication as a covariate removes variance associated with group differences, we performed additional analyses within the BPD group, medicated versus 
non-medicated, to check for possible effects of medication. It should be acknowledged that many researchers request patients to be free of medication several weeks before scanning, but it is not clear whether this assumption results in a normal brain state at the moment of scanning. Moreover, excluding those patients who refuse or cannot stop their medication contribute to a sampling bias. Furthermore, for some medication the washed out effect can last several months in the brain (Hafeman, Chang, Garrett, Sanders \& Phillips, 2012). A further complication is that different combinations of medication types are used in patients, which makes it virtually impossible to isolate the effect of a single agent. To gain more insight in and control as much as possible for the effect of medication, medication should be more adequately defined (type, number and dose) to have enough variance to add medication as a regressor in the analysis, nevertheless there is a risk to vary out relevant characteristics related to the disorder (Hafeman et al., 2012). A promising technique to get better insight in the effect of medication on brain activity is arterial spin labeling. This technique allows for measuring the absolute blood flow and provides a more quantitative estimate of blood perfusion (Wang, Chen, Fernandez-Seara \& Detre, 2011). Therefore it is able to determine the net effect of medication in the active state, accounting the information of medication effect on baseline perfusion.

\section{Gender}

Given the predominance of female BPD patients in mental health care samples (3:1; American Psychiatric Association, 2013), and the suggested different brain responses across gender concerning emotional processing (Whittle, Yucel, Yap \& Allen, 2011) and impulsivity (Li, Huang, Constable \& Sinha, 2006), the focus on female BPD patients in fMRI studies is understandable. Though, only investigating females will limit the generalizability of the results to males. Research indicates that the clinical picture across male and female BPD patients differs, in which females show more internalizing emotional behavior and males externalizing disinhibited and reactive aggressive behavior (Johnson et al., 2003). To clarify the potential role of gender in BPD future studies should compare male and female BPD patients.

\section{Avenues for future research}

\section{Psychotherapy: Changes in brain activation during the course of psychological treatment}

The effect of psychotherapy might be mediated by changes in brain activity. This suggests that psychological treatment inducing improvement of emotion regulation and impulsivity in BPD is accompanied with neural changes in limbic and prefrontal brain responses. Two studies focused on the normalization of the amygdala in response to passive viewing of negative pictures after dialectical behavior therapy (Goodman et al., 2014; Schnell \& Herpertz, 2007). The first study found a decreased activity in the right anterior cingulate 
cortex and left insula after 12-week in-patient dialectical behavior therapy in BPD patients (Schnell \& Herpertz, 2007). Furthermore, BPD patients exhibited greater activity in the anterior cingulate cortex, dorsolateral and dorsomedial prefrontal areas before dialectical behavior therapy while these differences were no longer shown after dialectical behavior therapy. This difference of change in the prefrontal activity was supported by the direct group comparison within BPD patients before and after dialectical behavior therapy. Moreover, the four responders to dialectical behavior therapy showed a reduced activity in the amygdala and hippocampus. The second study revealed that a reduction in activity of the amygdala in BPD patients following 12-month dialectical behavior therapy was associated with improved emotion regulation, measured with the Difficulty in Emotion Regulation Scale (Goodman et al., 2014). However, one should note that due to the small sample sizes of both studies, 6 and 11 per group respectively, the restricted statistical power might be too small to detect all effects and should be generalized with caution.

To date, only one study investigated symptom improvement after 12-week dialectical behavior therapy by means of an explicit form of emotion regulation (distraction; Winter et al., 2016). Research does show that distraction is successful in regulating emotional responses by guiding attention to a secondary task. In other words, distraction is an attentional emotion regulation strategy in which one focuses the attention away from the emotional content of the stimulus, and in this way decreases the emotional experience. Of note, BPD patients commonly use self-injurious behavior as an attentional distracting from emotions (regulation). In non-patients distraction, by means of memorize a letter/ digit string or a arithmetic task when presented with an emotional picture, led to increased activity in a widespread fronto-parietal network including lateral and medial prefrontal areas, the anterior cingulate and inferior parietal cortices, along with a decreased activity in the amygdala (Dorfel et al., 2014; Kanske, Heissler, Schonfelder, Bongers \& Wessa, 2011; McRae et al., 2010). A study examining the influence of emotional distraction on working memory suggested that compared to non-patients, BPD patients were more distracted by the emotional pictures during the working memory task indicated by stronger responses of the amygdala, hippocampus and insula (Krause-Utz et al., 2012). After dialectical behavior therapy BPD patients showed an decreased activity in the right supramarginal gyrus over time during distraction from negative versus neutral stimuli compared to non-patients as well as BPD patients who did not receive dialectical behavior therapy (Winter et al., 2016). This decrease correlated with reduced BPD symptom severity. Moreover, dialectical behavior therapy responders revealed a decreased activity in right perigenual anterior cingulate cortex following dialectical behavior therapy when viewing negative versus neutral stimuli, and a trend for increased activity in this brain area during distraction. Based on these results the authors suggest a shift from emotional to cognitive processing when presented with negative stimuli after successful dialectical behavior therapy and therefore less emotional vulnerability during distraction after improvement of BPD symptom severity. 
In a pilot study BPD patients performed an affective linguistic go/no-go task before and after transference-focused psychotherapy to investigate neural responses associated with, and predictive of, clinical improvement during behavioral inhibition in the context of negative emotional processing (Perez et al., 2016). BPD patients showed an increased activity in cognitive control areas including the dorsal anterior cingulate, dorsolateral prefrontal and frontopolar cortices, and a decreased activity in brain areas involved in emotional reactivity and semantic memory retrieval including the ventrolateral prefrontal cortex and hippocampus after transference-focused psychotherapy compared to before. Clinical improvement in constraint correlated positively with the dorsal anterior cingulate cortex, and in affective lability correlated negatively with amygdala/parahippocampal activity. Furthermore, post-treatment improvements in constraint were predicted by hypoactivity of the dorsal anterior cingulate cortex prior-treatment, and post-treatment improvements in affective lability were predicted by hypoactivity of the orbitofrontal cortex/ventral striatum prior-treatment. Consistent with the models of emotion and impulse regulation, these findings advocate that transference-focused psychotherapy is associated with increased cognitive control from the dorsal anterior cingulate cortex over limbic emotional reactivity indicated by reduced amygdala activity. This top-down frontolimbic activation pattern suggests that transference-focused psychotherapy might facilitate in symptom improvement (Perez et al., 2016).

To our knowledge these are the only studies examining the effects of psychological treatment on brain activity to date. Besides, the need that these studies should be replicated in larger sample sizes, several issues are of interest for future research. Individual differences of experiences in psychotherapy within the BPD group, affecting the ability and capacity to cognitively control emotions or impulses, might be reflected in a varying degree or ease to recruit the acquired brain areas (e.g. dorsolateral prefrontal cortex), or the engagement in various or familiarity with different regulation strategies. Furthermore, it should be acknowledged that besides the aforementioned distraction study (Krause-Utz et al., 2012) only cognitive reappraisal strategies (i.e. distancing and reinterpretation) were investigated across the studies examining emotion regulation in BPD (Koenigsberg et al., 2009a; Lang et al., 2012; Schulze et al., 2011) and it has not been investigated whether a certain emotion regulation strategy is more efficient than others for BPD patients (see also Chapter 2). It could be that distraction is useful when one could ignore the affective meaning of the stimulus for instance in situations that do not require memory of re-exposure is unlikely, while reappraisal might be more appropriate when the affective meaning must be addressed, manipulated and memorized and when re-exposure is likely, especially in personal relevant situations so future intrusions do not provoke the corresponding emotions. Subsequently, more research should be done to examine whether BPD patients differ in the neural correlates of different emotion regulation strategies and if these change after psychotherapy. Furthermore, changes in brain activity after other psychological treatments 
should be investigated. The above mentioned studies examined the effect of dialectical behavioral therapy and transference-focused psychotherapy, this line of research by itself has just begun, but one should also study the changes in brain activation during schema therapy and mentalized-based therapy. A forthcoming studies from our lab investigates whether the explicit regulation strategy of realizing being safe improves over the course of schema therapy and treatment-as-usual. Finally, insight should be generated in short and long term effects of psychotherapy in changes of brain activity by follow-up measurements months and years after treatment.

In sum, research on the mechanisms that mediate symptom improvement after psychotherapy is just in his infancy. Future research should examine how individual differences in brain activity are associated with responses to (successful) psychotherapy to investigate treatment outcome prediction, and identify changes of brain activity during emotion regulation strategies and impulse control after various psychotherapies to examine the treatment mechanism.

\section{Clinical application of real-time fMRI neurofeedback}

A helpful tool to improve cognitive control might be real-time fMRI. The ability to detect dynamic changes in local neural activation with real-time fMRI opens possibilities for studying the neural mechanisms of therapeutic interventions (e.g. cognitive behavioral therapy) and neurofeedback. Tracking changes in neural activation in real time and report back via visual feedback, enable participants to learn to voluntary control brain areas and might improve behavioral impairment (Johnston, Boehm, Healy, Goebel \& Linden, 2010). Down-regulating amygdala or insula activity involves a prefrontal-limbic coupling underlying successful emotion regulation, with real-time fMRI neurofeedback this potentially allows targeting brain networks of emotion processing. Effortful control by real-time fMRI neurofeedback of local brain activity of various cortical and subcortical areas relevant to emotion processing (e.g. amygdala, insula and anterior cingulate cortex) has been shown to be effective in modulating the appraisal of negative stimuli in non-patients (Caria, Sitaram, Veit, Begliomini \& Birbaumer, 2010; Johnston et al., 2010; Weiskopf et al., 2003; Zotev, Phillips, Young, Drevets \& Bodurka, 2013). A recent study investigated amygdala neurofeedback with the instruction to down-regulate in BPD patients (Paret et al., 2016). In addition, to provide information about temporal correlations of brain responses in other areas during emotion regulation of the target area (changes in functional connectivity) during the multi-session training, a resting-state scan was obtained at the beginning of each neurofeedback session. BPD patients were able to successful down-regulate the amygdala during negative stimuli via real-time $\mathrm{fMRI}$ neurofeedback training, though no transfer of successful down-regulation was found when no feedback was provided (i.e. no evidence of learning). Furthermore, task-related amygdala-ventromedial prefrontal cortex connectivity was increased when 
regulating versus viewing pictures, and functional connectivity during rest showed an increase in amygdala connectivity with the dorsolateral prefrontal cortex and decrease of connectivity with other limbic areas. Taken together, these finding demonstrate that modulation of the amygdala with real-time fMRI neurofeedback might improve emotion regulation and lead to a more healthy brain connectivity (Paret et al., 2016).Taking into account the results during passive viewing from current dissertation (Chapter 3) it might be interesting to investigate whether modulation of the anterior insula with real-time fMRI in BPD also results in improved emotion regulation and lead to a more healthy brain connectivity.

Given that the prefrontal cortex is considered to play a key role in top-down control of emotion and impulse control this might be a potential target for intervention to enhance efficacy of emotion regulation and reduce impulsivity. Based on the elevated response in the dorsolateral prefrontal cortex during passive viewing of negative pictures and impaired response during emotion regulation from current dissertation (Chapter 3), the dorsolateral prefrontal cortex might be a candidate area to develop real-time fMRI neurofeedback for BPD patients. Despite that real-time fMRI neurofeedback is often used as a training to guide participants towards the optimized mental strategies to control their brain activities, another approach could be to provide individuals with effective regulatory and inhibitory strategies and use the neurofeedback information to reinforce the feeling of control over their brain and in that way increase the participants' self-efficacy (Sarkheil et al., 2015). A study in nonpatients supported that real-time fMRI neurofeedback from the lateral prefrontal cortex facilitates modulation of amygdala activity during cognitive reappraisal of negative stimuli (Sarkheil et al., 2015).

Though, the learning and neural (interaction) mechanisms underlying the neurofeedback training effect are still unclear, neurofeedback for cognitive emotion regulation can be a highly relevant tool for cognitive interventions in psychotherapy. With respect to BPD, future studies should address specific emotion regulation instructions and dose (number of sessions)-response effects of neurofeedback. Furthermore, long-term effects of the training should be investigated by conducting follow-up examinations.

\section{Transcranial magnetic stimulation}

Another way of possibly improving cognitive control is transcranial magnetic stimulation. Transcranial magnetic stimulation is a magnetic method used to stimulate small regions of the brain. Since research demonstrates that impaired emotion regulation and impulsive behavior in BPD patients is driven by cortical regulatory and inhibitory deficits, transcranial magnetic stimulation can be used to test whether diminished cortical inhibition and/or enhanced excitation are presented in BPD. One study found evidence for an association between BPD and cortical inhibition deficits using transcranial magnetic stimulation 
(Barnow et al., 2009). A single case report (Arbabi, Hafizi, Ansari, Oghabian \& Hasani, 2013) and a pilot study (Cailhol et al., 2014) showed that changes in brain activity were associated with decrease in depression, negative affect, impulsivity, anger, general BPD and BPD severity after repetitive high-frequency transcranial magnetic stimulation treatment over the dorsolateral prefrontal cortex. Although these studies have small sample sizes they suggest a positive role for repetitive transcranial magnetic stimulation in management of BPD symptoms, and further evaluation of the use of transcranial magnetic stimulation would be beneficial. However, one should take into account that some target areas are not directly reachable, especially limbic areas including the amygdala, hippocampus, ventral striatum and insula. Concerning the dorsolateral prefrontal cortex it has often been suggested that this brain area plays an important role in cognitive control of emotions and impulses, therefore in clinical and treatment-oriented investigations it is one of the most frequently targeted areas for transcranial magnetic stimulation. It could be hypothesized that transcranial magnetic stimulation might influence cognitive control abilities by influencing fronto-limbic connectivity and restore top-down control of emotional responses. Furthermore, many psychotherapies in BPD involve learning emotion regulation strategies (or impulse control) to cognitive reappraise the affective meaning of symptom-eliciting situations in more neutral terms. Therefore, it would be interesting to investigate the effects of transcranial magnetic stimulation during emotion regulation and impulsivity tasks, and whether transcranial magnetic stimulation before treatment facilitates BPD patients to be more receptive in learning skills to cognitively reframe social-emotional triggers as nonthreatening.

\section{Concluding remarks}

In current dissertation we showed evidence for a general emotional sensitivity and impaired emotion regulation for negative stimuli in BPD. Additionally, BPD patients failed to show enduring effects in emotion regulation areas after the task. Furthermore, no support was shown for deficiencies of response inhibition under emotional processing. Interestingly, present studies point to indications of attentional processing problems in BPD during emotion regulation and impulse control. Understanding underlying neurocircuits might provide important insights in BPD and are valuable to improve psychological treatment. Effects of emotional sensitivity and in the inferior parietal lobe were not stimulus specific, whereas the top-down processes of emotion regulation and of the frontal eye fields were specific for negative stimuli. Overall, cluster-C patients showed activity that was inbetween BPD and non-patients, supporting a dimensional differentiation. Future research could elaborate on changes in brain activity during and after psychotherapy, real-time fMRI neurofeedback and transcranial magnetic stimulation to control brain activity and learn effective regulation and inhibition strategies. 


\section{References}

American Psychiatric Association. (2013). Diagnostic and Statistical Manual of Mental Disorder (5th ed.). Arlington, VA: American Psychiatric Association.

Arbabi, M., Hafizi, S., Ansari, S., Oghabian, M. A., \& Hasani, N. (2013). High frequency TMS for the management of borderline personality disorder: a case report. Asian Journal of Psychiatry, 6, 614-617. doi: 10.1016/j. ajp.2013.05.006

Arntz, A., Appels, C., \& Sieswerda, S. (2000). Hypervigilance in borderline disorder: a test with the emotional Stroop paradigm. Journal of Personality Disorders, 14, 366-373.

Arntz, A., Bernstein, D., Gielen, D., van Nieuwenhuyzen, M., Penders, K., Haslam, N., \& Ruscio, J. (2009). Taxometric evidence for the dimensional structure of cluster-C, paranoid, and borderline personality disorders. Journal of Personality Disorders, 23, 606-628. doi: 10.1521/pedi.2009.23.6.606

Arntz, A., Dreessen, L., Schouten, E., \&Weertman, A. (2004). Beliefs in personality disorders: a test with the personality disorder belief questionnaire. Behaviour Research and Therapy, 42, 1215-1225. doi: 10.1016/j.brat.2003.08.004

Arntz, A., \& van Genderen, H. (2009). Schema therapy for borderline personality disorder. Chichester: Wiley.

Barnow, S., Limberg, A., Stopsack, M., Spitzer, C., Grabe, H. J., Freyberger, H. J., \& Hamm, A. (2012). Dissociation and emotion regulation in borderline personality disorder. Psychological Medicine, 42, 783-794. doi: 10.1017/ S0033291711001917

Barnow, S., Volker, K. A., Moller, B., Freyberger, H. J., Spitzer, C., Grabe, H. J., \& Daskalakis, Z. J. (2009). Neurophysiological correlates of borderline personality disorder: a transcranial magnetic stimulation study. Biological Psychiatry, 65, 313-318. doi: 10.1016/j.biopsych.2008.08.016

Baskin-Sommers, A. R., Hooley, J. M., Dahlgren, M. K., Gonenc, A., Yurgelun-Todd, D. A., \& Gruber, S. A. (2015). Elevated preattentive affective processing in individuals with borderline personality disorder: a preliminary fMRI study. Frontiers in Psychology, 6, 1866. doi: 10.3389/fpsyg.2015.01866

Breiter, H. C., Etcoff, N. L., Whalen, P. J., Kennedy, W. A., Rauch, S. L., Buckner, R. L., .. Rosen, B. R. (1996). Response and habituation of the human amygdala during visual processing of facial expression. Neuron, 17, 875-887. doi: 10.1016/S0896-6273(00)80219-6

Buchheim, A., Erk, S., George, C., Kachele, H., Kircher, T., Martius, P., .. Walter, H. (2008). Neural correlates of attachment trauma in borderline personality disorder: a functional magnetic resonance imaging study. Psychiatry Research, 163, 223-235. doi: 10.1016/j.pscychresns.2007.07.001

Cailhol, L., Roussignol, B., Klein, R., Bousquet, B., Simonetta-Moreau, M., Schmitt, L., . . Birmes, P. (2014). Borderline personality disorder and rTMS: a pilot trial. Psychiatry Research, 216, 155-157. doi: 10.1016/j.psychres.2014.01.030

Caria, A., Sitaram, R., Veit, R., Begliomini, C., \& Birbaumer, N. (2010). Volitional control of anterior insula activity modulates the response to aversive stimuli. A real-time functional magnetic resonance imaging study. Biological Psychiatry, 68, 425-432. doi: 10.1016/j.biopsych.2010.04.020

Corbetta, M., Patel, G., \& Shulman, G. L. (2008). The reorienting system of the human brain: from environment to theory of mind. Neuron, 58, 306-324. doi: 10.1016/j.neuron.2008.04.017

Craig, A. D. (2009). How do you feel--now? The anterior insula and human awareness. Nature Reviews. Neuroscience, 10, 59-70. doi: 10.1038/nrn2555

Cullen, K. R., LaRiviere, L. L., Vizueta, N., Thomas, K. M., Hunt, R. H., Miller, M. J., . . Schulz, S. C. (2015). Brain activation in response to overt and covert fear and happy faces in women with borderline personality disorder. Brain Imaging and Behavior, 10, 319-331. doi: 10.1007/s11682-015-9406-4

Cullen, K. R., Vizueta, N., Thomas, K. M., Han, G. J., Lim, K. O., Camchong, J., ... Schulz, S. C. (2011). Amygdala functional connectivity in young women with borderline personality disorder. Brain Connectivity, 1, 61-71. doi: 10.1089/ brain.2010.0001

Cuthbert, B. N. (2014). The RDoC framework: facilitating transition from ICD/DSM to dimensional approaches that integrate neuroscience and psychopathology. World Psychiatry, 13, 28-35. doi: 10.1002/wps.20087

Daros, A. R., Zakzanis, K. K., \& Ruocco, A. C. (2013). Facial emotion recognition in borderline personality disorder. Psychological Medicine, 43, 1953-1963. doi: 10.1017/S0033291712002607

Delaveau, P., Jabourian, M., Lemogne, C., Guionnet, S., Bergouignan, L., \& Fossati, P. (2011). Brain effects of antidepressants in major depression: a meta-analysis of emotional processing studies. Journal of Affective Disorders, 130, 66-74. doi: 10.1016/j.jad.2010.09.032 
Doll, A., Sorg, C., Manoliu, A., Woller, A., Meng, C., Forstl, H., .. Riedl, V. (2013). Shifted intrinsic connectivity of central executive and salience network in borderline personality disorder. Frontiers in Human Neuroscience, 7, 727. doi: 10.3389/fnhum.2013.00727

Domes, G., Schulze, L., \& Herpertz, S. C. (2009). Emotion recognition in borderline personality disorder-a review of the literature. Journal of Personality Disorders, 23, 6-19. doi: 10.1521/pedi.2009.23.1.6

Domsalla, M., Koppe, G., Niedtfeld, I., Vollstadt-Klein, S., Schmahl, C., Bohus, M., \& Lis, S. (2014). Cerebral processing of social rejection in patients with borderline personality disorder. Social Cognitive and Affective Neuroscience, 9, 1789-1797. doi: 10.1093/scan/nst176

Donegan, N. H., Sanislow, C. A., Blumberg, H. P., Fulbright, R. K., Lacadie, C., Skudlarski, P., ... Wexler, B. E. (2003). Amygdala hyperreactivity in borderline personality disorder: implications for emotional dysregulation. Biological Psychiatry, 54, 1284-1293. doi: 10.1016/S0006-3223(03)00636-X

Dorfel, D., Lamke, J. P., Hummel, F., Wagner, U., Erk, S., \& Walter, H. (2014). Common and differential neural networks of emotion regulation by detachment, reinterpretation, distraction, and expressive suppression: a comparative fMRI investigation. Neurolmage, 101, 298-309. doi: 10.1016/j.neuroimage.2014.06.051

Driessen, M., Beblo, T., Mertens, M., Piefke, M., Rullkoetter, N., Silva-Saavedra, A., . . Woermann, F. G. (2004). Posttraumatic stress disorder and fMRI activation patterns of traumatic memory in patients with borderline personality disorder. Biological Psychiatry, 55, 603-611. doi: 10.1016/j.biopsych.2003.08.018

Dziobek, I., Preissler, S., Grozdanovic, Z., Heuser, I., Heekeren, H. R., \& Roepke, S. (2011). Neuronal correlates of altered empathy and social cognition in borderline personality disorder. Neurolmage, 57, 539-548. doi: 10.1016/j. neuroimage.2011.05.005

Ebner-Priemer, U. W., Badeck, S., Beckmann, C., Wagner, A., Feige, B., Weiss, I., . . Bohus, M. (2005). Affective dysregulation and dissociative experience in female patients with borderline personality disorder: a startle response study. Journal of Psychiatric Research, 39, 85-92. doi: 10.1016/j.jpsychires.2004.05.001

Ebner-Priemer, U. W., Mauchnik, J., Kleindienst, N., Schmahl, C., Peper, M., Rosenthal, M. Z., . . Bohus, M. (2009). Emotional learning during dissociative states in borderline personality disorder. Journal of Psychiatry \& Neuroscience, 34, 214-222.

Fischer, H., Furmark, T., Wik, G., \& Fredrikson, M. (2000). Brain representation of habituation to repeated complex visual stimulation studied with PET. NeuroReport, 11, 123-126. doi: 10.1097/00001756-200001170-00024

Frith, C. D., \& Frith, U. (2006). The neural basis of mentalizing. Neuron, 50, 531-534. doi: 10.1016/j.neuron.2006.05.001

Giesen-Bloo, J. H., Wachters, L. M., Schouten, E., \& Arntz, A. (2010). The Borderline Personality Disorder Severity IndexIV: psychometric evaluation and dimensional structure. Personality and Individual Differences, 49, 136-141. doi: 10.1016/j.paid.2010.03.023

Goodman, M., Carpenter, D., Tang, C. Y., Goldstein, K. E., Avedon, J., Fernandez, N., ... Hazlett, E. A. (2014). Dialectical behavior therapy alters emotion regulation and amygdala activity in patients with borderline personality disorder. Journal of Psychiatric Research, 57, 108-116. doi: 10.1016/j.jpsychires.2014.06.020

Gross, J. J. (2007). Handbook of Emotion Regulation. New York, NY: Guilford Publications

Hafeman, D. M., Chang, K. D., Garrett, A. S., Sanders, E. M., \& Phillips, M. L. (2012). Effects of medication on neuroimaging findings in bipolar disorder: an updated review. Bipolar Disorders, 14, 375-410. doi: 10.1111/j.13995618.2012.01023.x

Hazlett, E. A., Zhang, J., New, A. S., Zelmanova, Y., Goldstein, K. E., Haznedar, M. M., ... Chu, K. W. (2012). Potentiated amygdala response to repeated emotional pictures in borderline personality disorder. Biological Psychiatry, 72 448-456. doi: 10.1016/j.biopsych.2012.03.027

Herpertz, S. C., Dietrich, T. M., Wenning, B., Krings, T., Erberich, S. G., Willmes, K., . . Sass, H. (2001). Evidence of abnormal amygdala functioning in borderline personality disorder: a functional MRI study. Biological Psychiatry, 50, 292-298. doi: 10.1016/S0006-3223(01)01075-7

Johnson, D. M., Shea, M. T., Yen, S., Battle, C. L., Zlotnick, C., Sanislow, C. A., .. Zanarini, M. C. (2003). Gender differences in borderline personality disorder: findings from the Collaborative Longitudinal Personality Disorders Study. Comprehensive Psychiatry, 44, 284-292. doi: 10.1016/S0010-440X(03)00090-7

Johnston, S. J., Boehm, S. G., Healy, D., Goebel, R., \& Linden, D. E. (2010). Neurofeedback: a promising tool for the selfregulation of emotion networks. Neurolmage, 49, 1066-1072. doi: 10.1016/j.neuroimage.2009.07.056

Kaiser, D., Jacob, G. A., Domes, G., \& Arntz, A. (2016). Attentional bias for emotional stimuli in borderline personality disorder: a meta-analysis. Psychopathology. doi: 10.1159/000448624 
Kamphausen, S., Schroder, P., Maier, S., Bader, K., Feige, B., Kaller, C. P., .. Tüscher, O. (2013). Medial prefrontal dysfunction and prolonged amygdala response during instructed fear processing in borderline personality disorder. The World Journal of Biological Psychiatry, 14, 307-318, S301-304. doi: 10.3109/15622975.2012.665174

Kanske, P., Heissler, J., Schonfelder, S., Bongers, A., \&Wessa, M. (2011). How to regulate emotion? Neural networks for reappraisal and distraction. Cerebral Cortex, 21, 1379-1388. doi: 10.1093/cercor/bhq216

King-Casas, B., Sharp, C., Lomax-Bream, L., Lohrenz, T., Fonagy, P., \& Montague, P. R. (2008). The rupture and repair of cooperation in borderline personality disorder. Science, 321, 806-810. doi: 10.1126/science.1156902

Koenigsberg, H. W., Denny, B. T., Fan, J., Liu, X., Guerreri, S., Mayson, S. J., ... Siever, L. J. (2014). The neural correlates of anomalous habituation to negative emotional pictures in borderline and avoidant personality disorder patients. The American Journal of Psychiatry, 171, 82-90. doi: 10.1176/appi.ajp.2013.13070852

Koenigsberg, H. W., Fan, J., Ochsner, K. N., Liu, X., Guise, K. G., Pizzarello, S., .. Siever, L. J. (2009a). Neural correlates of the use of psychological distancing to regulate responses to negative social cues: a study of patients with borderline personality disorder. Biological Psychiatry, 66, 854-863. doi: 10.1016/j.biopsych.2009.06.010

Koenigsberg, H. W., Siever, L. J., Lee, H., Pizzarello, S., New, A. S., Goodman, M., . . Prohovnik, I. (2009b). Neural correlates of emotion processing in borderline personality disorder. Psychiatry Research, 172, 192-199. doi: 10.1016/j.pscychresns.2008.07.010

Korzekwa, M. I., Dell, P. F., Links, P. S., Thabane, L., \& Fougere, P. (2009). Dissociation in borderline personality disorder: a detailed look. Journal of Trauma \& Dissociation, 10, 346-367. doi: 10.1080/15299730902956838

Krause-Utz, A., Elzinga, B. M., Oei, N. Y., Paret, C., Niedtfeld, I., Spinhoven, P., ... Schmahl, C. (2014a). Amygdala and dorsal anterior cingulate connectivity during an emotional working memory task in borderline personality disorder patients with interpersonal trauma history. Frontiers in Human Neuroscience, 8, 848. doi: 10.3389/ fnhum.2014.00848

Krause-Utz, A., Oei, N. Y., Niedtfeld, I., Bohus, M., Spinhoven, P., Schmahl, C., \& Elzinga, B. M. (2012). Influence of emotional distraction on working memory performance in borderline personality disorder. Psychological Medicine, 42, 2181-2192. doi: 10.1017/S0033291712000153

Krause-Utz, A., Veer, I. M., Rombouts, S. A., Bohus, M., Schmahl, C., \& Elzinga, B. M. (2014b). Amygdala and anterior cingulate resting-state functional connectivity in borderline personality disorder patients with a history of interpersonal trauma. Psychological Medicine, 44, 2889-2901. doi: 10.1017/S0033291714000324

Lang, S., Kotchoubey, B., Frick, C., Spitzer, C., Grabe, H. J., \& Barnow, S. (2012). Cognitive reappraisal in traumaexposed women with borderline personality disorder. Neurolmage, 59, 1727-1734. doi: 10.1016/j. neuroimage.2011.08.061

Lazarus, S. A., Cheavens, J. S., Festa, F., \& Rosenthal, M. Z. (2014). Interpersonal functioning in borderline personality disorder: a systematic review of behavioral and laboratory-based assessments. Clinical Psychology Review, 34, 193-205. doi: 10.1016/j.cpr.2014.01.007

Li, C. S., Huang, C., Constable, R. T., \& Sinha, R. (2006). Gender differences in the neural correlates of response inhibition during a stop signal task. Neurolmage, 32, 1918-1929. doi: 10.1016/j.neuroimage.2006.05.017

Lieb, K., Zanarini, M. C., Schmahl, C., Linehan, M. M., \& Bohus, M. (2004). Borderline personality disorder. Lancet, 364, 453-461. doi: 10.1016/S0140-6736(04)16770-6

Lobbestael, J., Arntz, A., \& Bernstein, D. P. (2010). Disentangling the relationship between different types of childhood maltreatment and personality disorders. Journal of Personality Disorders, 24, 285-295. doi: 10.1521/ pedi.2010.24.3.285

Ma, Y. (2015). Neuropsychological mechanism underlying antidepressant effect: a systematic meta-analysis. Molecular Psychiatry, 20, 311-319. doi: 10.1038/mp.2014.24

McGlashan, T. H., Grilo, C. M., Skodol, A. E., Gunderson, J. G., Shea, M. T., Morey, L. C., . . Stout, R. L. (2000). The Collaborative Longitudinal Personality Disorders Study: baseline Axis I/II and II/II diagnostic co-occurrence. Acta Psychiatrica Scandinavica, 102, 256-264. doi: 10.1034/j.1600-0447.2000.102004256.x

McRae, K., Hughes, B., Chopra, S., Gabrieli, J. D., Gross, J. J., \& Ochsner, K. N. (2010). The neural bases of distraction and reappraisal. Journal of Cognitive Neuroscience, 22, 248-262. doi: 10.1162/jocn.2009.21243

Mitchell, A. E., Dickens, G. L., \& Picchioni, M. M. (2014). Facial emotion processing in borderline personality disorder: a systematic review and meta-analysis. Neuropsychological Review, 24, 166-184. doi: 10.1007/s11065-014-9254-9

National Institute of Mental Health. (2008). The National Institute of Mental Health Strategic Plan, from http://www. nimh.nih.gov/about/strategic-planning-reports/index.shtml 
Niedtfeld, I., Kirsch, P., Schulze, L., Herpertz, S. C., Bohus, M., \& Schmahl, C. (2012). Functional connectivity of painmediated affect regulation in borderline personality disorder. PLOS One, 7, e33293. doi: 10.1371/journal. pone.0033293

Paret, C., Kluetsch, R., Zaehringer, J., Ruf, M., Demirakca, T., Bohus, M., .. Schmahl, C. (2016). Alterations of amygdalaprefrontal connectivity with real-time fMRI neurofeedback in BPD patients. Social Cognitive and Affective Neuroscience. doi: 10.1093/scan/nsw016

Perez, D. L., Vago, D. R., Pan, H., Root, J., Tuescher, O., Fuchs, B. H., . . Stern, E. (2016). Frontolimbic neural circuit changes in emotional processing and inhibitory control associated with clinical improvement following transference-focused psychotherapy in borderline personality disorder. Psychiatry and Clinical Neurosciences, 70, 51-61. doi: 10.1111/pcn.12357

Pretzer, J. (1990). Borderline personality disorder. In A. T. Beck \& A. Freeman (Eds.), Cognitive therapy of personality disorders (pp. 176-207). New York/London: Guilford Press.

Radaelli, D., Poletti, S., Dallaspezia, S., Colombo, C., Smeraldi, E., \& Benedetti, F. (2012). Neural responses to emotional stimuli in comorbid borderline personality disorder and bipolar depression. Psychiatry Research, 203, 61-66. doi: 10.1016/j.pscychresns.2011.09.010

Sanislow, C. A., Grilo, C. M., Morey, L. C., Bender, D. S., Skodol, A. E., Gunderson, J. G., . . McGlashan, T. H. (2002). Confirmatory factor analysis of DSM-IV criteria for borderline personality disorder: findings from the collaborative longitudinal personality disorders study. The American Journal of Psychiatry, 159, 284-290.

Sarkheil, P., Zilverstand, A., Kilian-Hutten, N., Schneider, F., Goebel, R., \& Mathiak, K. (2015). fMRI feedback enhances emotion regulation as evidenced by a reduced amygdala response. Behavioural Brain Research, 281, 326-332. doi: 10.1016/j.bbr.2014.11.027

Saxe, R., Xiao, D. K., Kovacs, G., Perrett, D. I., \& Kanwisher, N. (2004). A region of right posterior superior temporal sulcus responds to observed intentional actions. Neuropsychologia, 42, 1435-1446. doi: 10.1016/j. neuropsychologia.2004.04.015

Scherpiet, S., Bruhl, A. B., Opialla, S., Roth, L., Jancke, L., \& Herwig, U. (2014). Altered emotion processing circuits during the anticipation of emotional stimuli in women with borderline personality disorder. European Archives of Psychiatry and Clinical Neuroscience, 264, 45-60. doi: 10.1007/s00406-013-0444-x

Schnell, K., \& Herpertz, S. C. (2007). Effects of dialectic-behavioral-therapy on the neural correlates of affective hyperarousal in borderline personality disorder. Journal of Psychiatric Research, 41, 837-847. doi: 10.1016/j. jpsychires.2006.08.011

Schulze, L., Domes, G., Kruger, A., Berger, C., Fleischer, M., Prehn, K., ... Herpertz, S. C. (2011). Neuronal correlates of cognitive reappraisal in borderline patients with affective instability. Biological Psychiatry, 69, 564-573. doi: 10.1016/j.biopsych.2010.10.025

Shamay-Tsoory, S. G. (2011). The neural bases for empathy. The Neuroscientist, 17, 18-24. doi: $10.1177 / 1073858410379268$

Sierra, M., \& Berrios, G. E. (1998). Depersonalization: neurobiological perspectives. Biological Psychiatry, 44, 898-908. doi: 10.1016/S0006-3223(98)00015-8

Soloff, P. H., White, R., Omari, A., Ramaseshan, K., \& Diwadkar, V. A. (2015). Affective context interferes with brain responses during cognitive processing in borderline personality disorder: fMRI evidence. Psychiatry Research, 233, 23-35. doi: 10.1016/j.pscychresns.2015.04.006

Trull, T. J., Distel, M. A., \& Carpenter, R. W. (2011). DSM-5 Borderline personality disorder: at the border between a dimensional and a categorical view. Current Psychiatry Reports, 13, 43-49. doi: 10.1007/s11920-010-0170-2

von Ceumern-Lindenstjerna, I. A., Brunner, R., Parzer, P., Mundt, C., Fiedler, P., \& Resch, F. (2010). Attentional bias in later stages of emotional information processing in female adolescents with borderline personality disorder. Psychopathology, 43, 25-32. doi: 10.1159/000255960

Wagner, A. W., \& Linehan, M. M. (1999). Facial expression recognition ability among women with borderline personality disorder: implications for emotion regulation? Journal of Personality Disorders, 13, 329-344. doi: 10.1521/pedi.1999.13.4.329

Wagner, D. D., \& Heatherton, T. F. (2013). Self-regulatory depletion increases emotional reactivity in the amygdala. Social Cognitive and Affective Neuroscience, 8, 410-417. doi: 10.1093/scan/nss082

Wang, D. J., Chen, Y., Fernandez-Seara, M. A., \& Detre, J. A. (2011). Potentials and challenges for arterial spin labeling in pharmacological magnetic resonance imaging. The Journal of Pharmacology and Experimental Therapeutics, 337, 359-366. doi: 10.1124/jpet.110.172577 
Weiskopf, N., Veit, R., Erb, M., Mathiak, K., Grodd, W., Goebel, R., \& Birbaumer, N. (2003). Physiological self-regulation of regional brain activity using real-time functional magnetic resonance imaging (fMRI): methodology and exemplary data. Neurolmage, 19, 577-586.

Whittle, S., Yucel, M., Yap, M. B., \& Allen, N. B. (2011). Sex differences in the neural correlates of emotion: evidence from neuroimaging. Biological Psychology, 87, 319-333. doi: 10.1016/j.biopsycho.2011.05.003

Widiger, T. A., \& Trull, T. J. (2007). Plate tectonics in the classification of personality disorder: shifting to a dimensional model. The American Psychologist, 62, 71-83. doi: 10.1037/0003-066X.62.2.71

Winter, D., Krause-Utz, A., Lis, S., Chiu, C. D., Lanius, R. A., Schriner, F., .. Schmahl, C. (2015). Dissociation in borderline personality disorder: disturbed cognitive and emotional inhibition and its neural correlates. Psychiatry Research, 233, 339-351. doi: 10.1016/j.pscychresns.2015.05.018

Winter, D., Niedtfeld, I., Schmitt, R., Bohus, M., Schmahl, C., \& Herpertz, S. C. (2016). Neural correlates of distraction in borderline personality disorder before and after dialectical behavior therapy. European Archives of Psychiatry and Clinical Neuroscience. doi: 10.1007/s00406-016-0689-2

Wolf, R. C., Sambataro, F., Vasic, N., Schmid, M., Thomann, P. A., Bienentreu, S. D., \& Wolf, N. D. (2011). Aberrant connectivity of resting-state networks in borderline personality disorder. Journal of Psychiatry \& Neuroscience, 36, 402-411. doi: 10.1503/jpn.100150

Zanarini, M. C., Frankenburg, F. R., Dubo, E. D., Sickel, A. E., Trikha, A., Levin, A., \& Reynolds, V. (1998a). Axis I comorbidity of borderline personality disorder. The American Journal of Psychiatry, 155, 1733-1739.

Zanarini, M. C., Frankenburg, F. R., Dubo, E. D., Sickel, A. E., Trikha, A., Levin, A., \& Reynolds, V. (1998b). Axis II comorbidity of borderline personality disorder. Comprehensive Psychiatry, 39, 296-302. doi: 10.1016/50010-440X(98)90038-4

Zanarini, M. C., Ruser, T., Frankenburg, F. R., \& Hennen, J. (2000). The dissociative experiences of borderline patients. Comprehensive Psychiatry, 41, 223-227. doi: 10.1016/S0010-440X(00)90051-8

Zimmerman, M., \& Mattia, J. I. (1999). Axis I diagnostic comorbidity and borderline personality disorder. Comprehensive Psychiatry, 40, 245-252. doi: 10.1016/S0010-440X(99)90123-2

Zotev, V., Phillips, R., Young, K. D., Drevets, W. C., \& Bodurka, J. (2013). Prefrontal control of the amygdala during real-time fMRI neurofeedback training of emotion regulation. PLoS One, 8, e79184. doi: 10.1371/journal. pone.0079184 


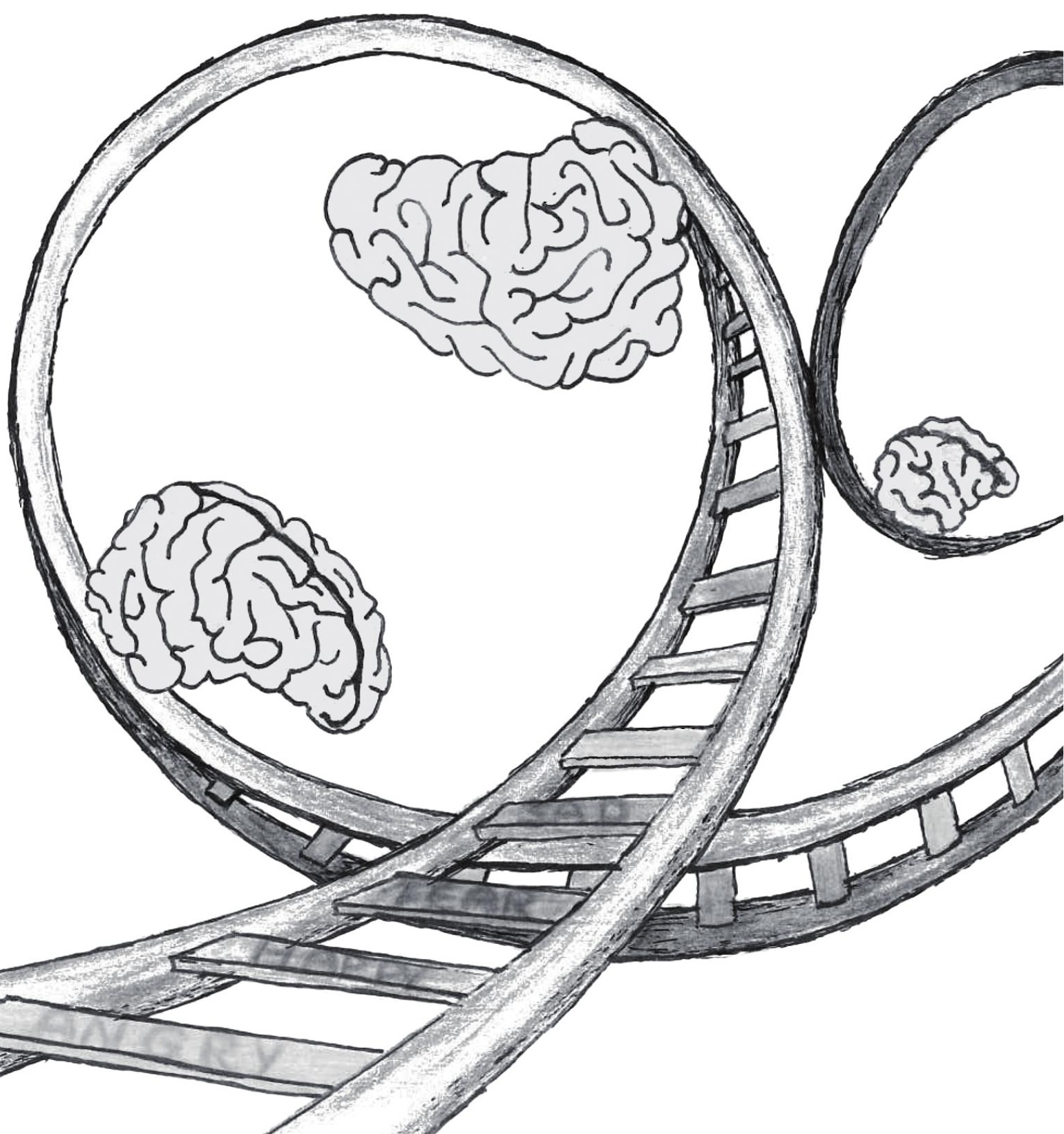




\section{Valorization Addendum}



The work presented in the current dissertation aimed to investigate borderline personality disorder (BPD) uniqueness of neural correlates of emotional sensitivity, emotion regulation and impulsivity, especially with regard to stimulus category specificity and diagnosis specificity. This valorization addendum focusses on a broader societal and economic context, it discusses the societal and economic relevance, gives an overview of the target groups for whom our research findings might be relevant, discusses possible activities and services, and innovative aspects resulting from the research.

\section{Societal and Economic relevance}

BPD is a common mental disorder that is associated with negative consequences from societal and economic points of view. BPD imposes a substantial impact on those suffering from BPD, their friends and families (including possible children), the public health systems and society. Apart from the severe functional impairments, BPD is considered to be difficult to treat and requires more treatment than patients with other disorders. Despite that effective treatments are available now, not every patient recovers fully, and treatments are lengthy and costly. Therefore, BPD is considered to be one of the most expensive psychiatric disorders. This economic burden of BPD is due to direct costs including all medical costs (e.g. hospital days, emergency room visits and psychotherapy), and indirect costs that occur secondary to the disorder (e.g. production loss and work absence). The overall annual societal costs in the Dutch population were estimated at 16.852 euro (price level 2000) per patient (van Asselt, Dirksen, Arntz \& Severens, 2007), of which more than 50\% were indirect costs. Although the current dissertation does not directly contribute to decreasing costs it contributes to increasing insight into underlying mechanisms of emotional sensitivity, emotion regulation and impulsivity and point towards new targets for treatment. For example, the dorsolateral prefrontal cortex is considered to play an important role in top-down control and might be a potential target for real-time fMRI neurofeedback and transcranial magnetic stimulation, both potential tools to improve cognitive control. Furthermore, the effect of psychotherapy might be mediated by changes in brain activity. Therefore, understanding how brain activity is associated with (successful) psychotherapy (treatment response predication), and identify changes of brain activity after psychotherapy (treatment mechanism) is of great relevance. Next, specific components can be added or removed to make the treatment more efficient and effective. A forthcoming series of studies from our lab that will contribute to this aim investigates changes of brain activity over the course of schema therapy and treatment-as-usual. Finally, cost-effectiveness of individual schema therapy has been demonstrated previously in the Netherlands in borderline and other personality disorders (Bamelis, Arntz, Wetzelaer, Verdoorn \& Evers, 2015; van Asselt, 2008). The BPD patients included in the studies of the current dissertation were recruited in the context of an international multicenter randomized control trial investigating the clinical 
effectiveness, cost-effectiveness and cost-utility of group schema therapy as compared to treatment-as-usual (Wetzelaer et al., 2014).

\section{Target groups for whom our findings might be relevant}

The findings of this dissertation might be relevant for several target groups: 1) researchers in the field of BPD to verify and refine theoretical models, 2) health care professionals as the gained knowledge might provide new leads to effective treatment, 3) patients suffering from BPD and their family and friends, and 4) society to reduce the economic costs. Together, to strive for better understanding of BPD and psychotherapy with their possible underlying mechanisms of change, we might be able to bridge existing gaps between research and theory on the one hand and clinical practice on the other hand.

\section{Activities and services}

This dissertation is driven by the motivation to understand and help BPD patients more effectively. Ultimately validation of clinical theories and insight in the underlying mechanisms might be used to improve treatment outcomes and novel treatment techniques. One can train emotion regulation strategies or control and alter patterns of brain activity, using for instance real-time fMRI neurofeedback and transcranial magnetic stimulation. When successful, these techniques might be integrated in treatment modules. However, to date it is too premature for workshops (in the context of scientific conferences) and training programs to teach health care professionals specific techniques.

\section{Innovation}

Abnormalities in the regulation of emotions or inhibition of impulses in BPD have been linked to altered fronto-limbic brain responses. Therefore empirical knowledge on BPD uniqueness of neural correlates of emotional sensitivity, emotion regulation and impulsivity can inform us about the underlying mechanisms and possible targets for treatment. Chapter 2 sheds a critical light on the methodology and important unanswered issues of fMRI studies involving emotional sensitivity, emotion regulation and impulsivity in BPD. In order to improve our understanding of BPD the studies conducted in chapters 3 and 4 address novel research questions build upon a couple of the issues pointed out in the critical review of chapter 2. More specifically, we extended previous research by adding positive and erotic stimuli to the traditional negative and neutral stimuli to examine stimulus category specificity across stimuli differing in their emotional content. As previous research mostly involves negative stimuli it is unclear whether emotional reactivity also holds for positive stimuli, pointing to a general emotional reactivity. Additionally, given high rates of childhood sexual traumatization and relationship problems are often reported in BPD, erotic 
stimuli were added. Furthermore, because of high rates of comorbid Axis I and II disorders within BPD it remains unclear whether findings are BPD diagnosis specific or characteristic of psychopathology in general. By adding a clinical control group of Cluster-C personality disorder patients we examined diagnosis specificity compared to other personality disorders. Finally, the statistical power was increased, compared to previous studies, by increasing the sample size by recruiting participants from multiple international centers. In chapter 5 we included repeated resting-state enabling the investigation of post-task brain changes. Besides the studies showing altered amygdala-prefrontal functional connectivity during induction of negative emotions and task-free resting-state, this is the first study to examine the enduring effects of an emotion regulation task on amygdala resting-state functional connectivity. An objective for future research will be the use of longitudinal designs predicting clinical outcomes.

Although not an immediate consequence for treatment these findings do contribute to a better clinical understanding of BPD, and how BPD compares to other personality disorders. The results relating to stimulus category specificity indicate a general emotional sensitivity as they were also present for positive stimuli, whereas emotion regulation was specific for negative stimuli. The latter however makes sense, considering that regulation of positive emotions is in most cases undesirable. The findings concerning response inhibition showed a general responsivity across stimulus categories in the inferior parietal lobule, while effects in the frontal eye fields were specific for negative stimuli. This might suggest that if top-down modulation does not work on the perceptional input channel at the level of the inferior parietal lobule, in BPD this must happen in the output channel of the frontal eye fields.

Furthermore, no significant differences regarding emotion generation and emotion regulation brain areas and impulse control of social emotional stimuli were found for the comparison BPD patients versus Cluster-C personality disorder patients, indicating that these results were not diagnosis specific for BPD. Linearity analyses showed responses of the Cluster-C personality disorder patients in-between the non-patients and BPD patients. This supports the notion of a dimensional approach rather than a categorical approach, in which Cluster-C personality disorder patients exhibit emotional and interpersonal features in common with BPD, yet weaker.

\section{Schedule and implementation}

This work has been conducted in the program "Open Research Area in the Social Sciences", in which participants were recruited in two sites in the Netherlands (Maastricht and Heerlen) and three sites in Germany (Freiburg, Lübeck, and Hamburg). Therefore to perform the present international multicenter fMRI studies, our research group developed international relationships with Freiburg and Lübeck. 
The outcome of the studies described in this dissertation will be communicated within scientific community by international peer-reviewed journal publications and conference presentations. Communication of the findings outside the scientific field will be done through the media.

\section{References}

Bamelis, L.L, Arntz, A., Wetzelaer, P., Verdoorn \& Everes, S.M. (2015). Economic evaluation of schema therapy and clarification-oriented psychotherapy for personality disorders: a multicenter, randomized controlled trial. Journal of Clinical Psychiatry, 76, e1432-1440. doi: 10.4088/JCP.14m09412.

van Asselt, A. D., Dirksen, C. D., Arntz, A., Giesen-Bloo, J.H., van Dyck, R., ... Severens, J. L. (2007). Out-patient psychotherapy for borderline personality disorder: cost-effectiveness of schema-focused therapy $v$. transference-focused psychotherapy. British Journal of Psychiatry, 192, 450-457. doi: 10.1192/bjp.bp.106.033597.

van Asselt, A. D., Dirksen, C. D., Arntz, A., \& Severens, J. L. (2007). The cost of borderline personality disorder: societal cost of illness in BPD-patients. European Psychiatry, 22, 354-361. doi: 10.1016/j.eurpsy.2007.04.001.

Wetzelaer, P., Farrell, J., Evers, S., Jacob, G. A., Lee, C. W., Brand, O., . . Arntz, A. (2014). Design of an international multicentre RCT on group schema therapy for borderline personality disorder. BMC Psychiatry, 14, 319. doi: 10.1186/s12888-014-0319-3. 



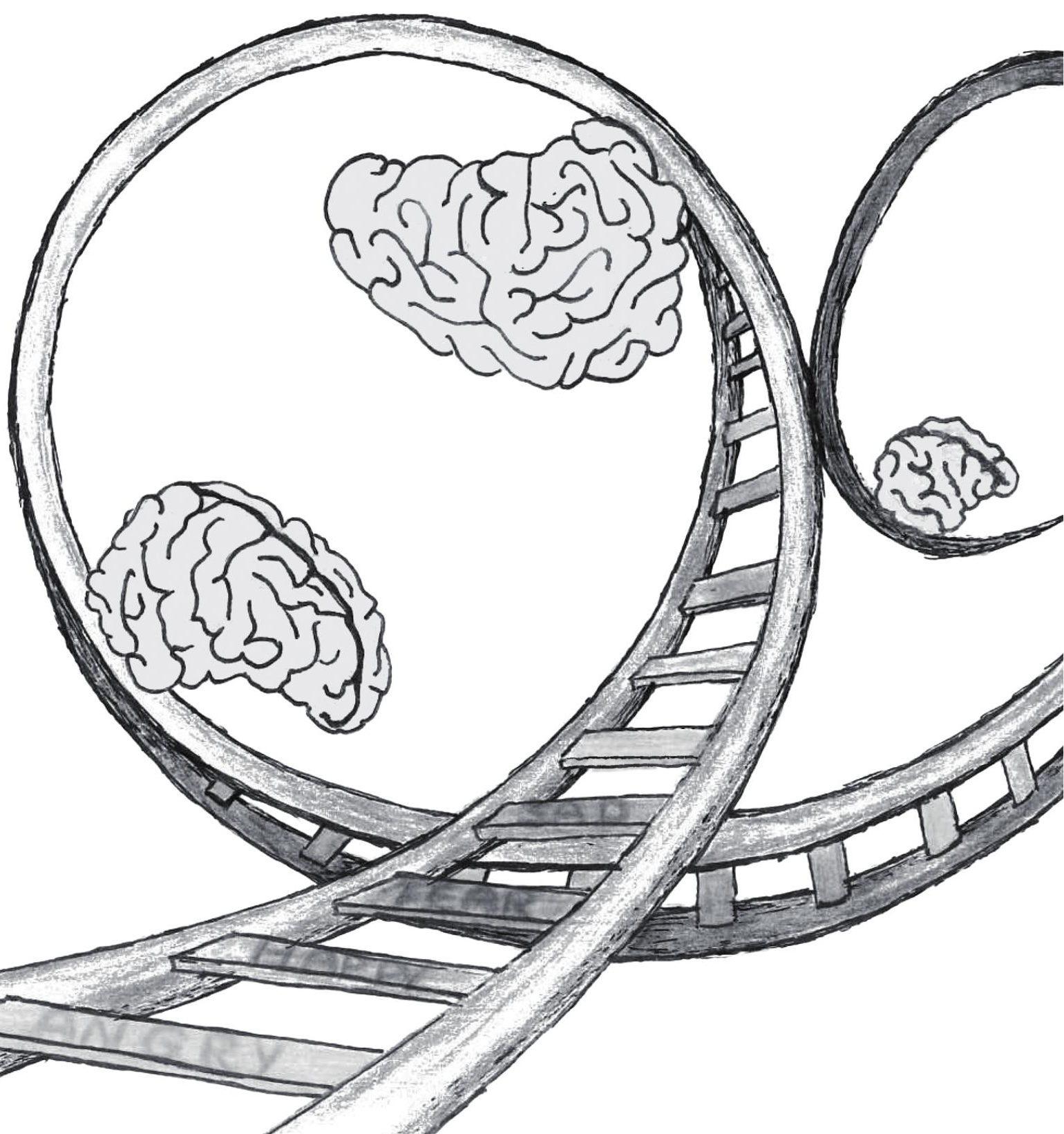


Samenvatting

Dutch Summary 

Borderline persoonlijkheidsstoornis (BPS) is een ernstige psychiatrische stoornis, die 1-3\% van de algemene bevolking treft, en in de geestelijke gezondheidszorg (GGZ) naar schatting 10\% van de poliklinische patiënten en 20\% van de opgenomen patiënten. BPS stelt uitdagingen voor mensen met BPS, maar ook voor hun families en vrienden, en beïnvloedt het functioneren in het sociale domein en werkdomein. Naast de gevolgen als werkloosheid en arbeidsongeschiktheid, wordt BPS beschouwd als moeilijk behandelbaar. BPS patiënten maken intensief gebruik van behandeling en andere geestelijke gezondheidszorgvoorzieningen. Deze combinatie leidt tot een last voor maatschappelijke systemen en hoge kosten voor de geestelijke gezondheidszorg en voor de samenleving in het algemeen.

In de Diagnostic and Statistical Manual of Mental Disorders (DSM) worden de belangrijkste kenmerken van BPS beschreven als een patroon van instabiliteit in interpersoonlijke relaties, emoties, zelfbeeld, en impulsief gedrag. Verder worden BPS patiënten gekenmerkt door extreme angst voor verlating en afwijzing, gevoel van leegte, herhaalde zelfverwonding, suïcidale neigingen en reactieve agressie. Oorzakelijke factoren die bijdragen aan de ontwikkeling van BPS zijn slechts ten dele bekend, maar er wordt aangenomen dat het gaat om een interactie tussen genetische factoren en omgevingsfactoren, zoals fysiek en seksueel misbruik of emotionele verwaarlozing tijdens de kindertijd.

Toonaangevende theorieën over de etiologie van BPS stellen dat BPS het best kan worden begrepen als een emotie-disregulatie stoornis, veroorzaakt door een verhoogde emotionele gevoeligheid enerzijds en een onvermogen om emotionele reacties te reguleren anderzijds. Het onvermogen om emotionele reacties te reguleren kan vervolgens leiden tot impulsief gedrag, ook kenmerkend voor BPS, vooral onder emotionele omstandigheden. Emotionele gevoeligheid kan worden gedefinieerd als een lage drempel voor het detecteren van of reageren op emotionele stimuli, of een hoge kans om stimuli als emotioneel te ervaren. Dit kan leiden tot overbewustzijn van emotionele stimuli en meer frequente en intense emotionele ervaringen. Emotieregulatie verwijst naar het vermogen van individuen om de expressie en ervaring van emoties te beïnvloeden. Dit kan worden bereikt door middel van cognitieve controle, zoals her-evaluatie en herinterpretatie, waardoor de intensiteit van de emotionele ervaring vermindert. Impulsiviteit bij BPS patiënten wordt vaak gediagnosticeerd binnen het gedragsdomein en gedefinieerd als snelle en ongeplande reacties zonder na te denken over de gevolgen (impulscontrole of responsinhibitie). Dit patroon van impulsiviteit in BPS wordt gekenmerkt door fysiek zelfdestructief gedrag, zoals zelfverwonding en zelfmoordpogingen, en in meer algemene vormen van impulsiviteit, zoals buitensporige uitgaven van geld, winkeldiefstal, onveilige seks, drugs of alcoholmisbruik, vreetbuien, roekeloos rijden, woede uitbarsting en reactieve agressie.

In de afgelopen jaren hebben een toenemend aantal neuroimaging studies met betrekking tot neurobiologische oorzaken van BPS met behulp van emotieprovocatie 
paradigma's hersengebieden en netwerken geïdentificeerd die geassocieerd worden met BPS. Veel van deze studies wijzen op een karakteristieke respons in het fronto-limbische netwerk, bestaande uit een verhoogde bottom-up limbische activiteit (amygdala en insula) en verminderde top-down prefrontale activiteit (dorsolaterale, dorsomediale, ventrolaterale prefrontale cortices en dorsale anterior cingulate cortex) bij BPS patiënten in reactie op emotionele stimuli. Omdat limbische gebieden over het algemeen geassocieerd worden met het detecteren en genereren van emoties en prefrontale gebieden met cognitieve controle processen zoals regulatie en inhibitie, worden deze bevindingen geïnterpreteerd als zijnde in overeenstemming met de emotie-disregulatie hypothese van BPS.

In Hoofdstuk 2 werd een gedetailleerde inleiding van het neurobiologische model van BPS gegeven, waarin fMRI studies naar emotionele gevoeligheid, emotieregulatie en impulsiviteit in BPS werden samengevat. Bovendien werd een kritische blik geworpen op de methodologie en de resultaten van fMRI studies naar emotionele gevoeligheid, emotieregulatie en impulsiviteit in BPS in reactie op negatieve stimuli. Dit overzicht geeft aan dat een hogere emotionele gevoeligheid, verstoorde emotieregulatie en problemen met impulscontrole in BPS het best kan worden begrepen in termen van een verminderde inhibitie van de prefrontale gebieden op de limbische gebieden. Huidige neuroimaging bevindingen zijn echter minder overtuigend en consistent als eerder verondersteld, er zijn sterke overeenkomsten maar ook belangrijke verschillen in resultaten van verschillende onderzoeken. Aanwijzingen voor een verhoogde amygdala activiteit was het meest consistent tijdens provocatie van emotionele gevoeligheid bij BPS patiënten. De meest consistente bevinding met betrekking tot emotieregulatie bij BPS patiënten was een verminderde activiteit van de anterior cingulate cortex, terwijl de mediale en dorsolaterale prefrontale gebieden variabele activiteit toonden. Met betrekking tot impulsiviteit was verminderde activiteit van de inhiberende hersengebieden in BPS patiënten inconsistent. Voor replicaties van de resultaten van eerdere studies en het beantwoorden van een aantal onbeantwoorde kwesties zoals generalisatie en specificiteit, moet bij toekomstig onderzoek rekening worden gehouden met de beperkte statistische power van eerdere studies en moet de steekproef worden verhoogd. Bovendien moet toekomstig onderzoek zich richten op BPS-specificiteit met betrekking tot de (uitgestelde) gewenningseffecten van de amygdala, verschillende emotieregulatie strategieën, manipulatie van zelf-regulatie, het gebruik van medicatie en sekseverschillen. Tenslotte wordt uitbreiding van het type stimuli (bijv. positief en niet-sociaal) en het belang van een klinische controlegroep besproken. Een beter begrip van de onderliggende neurale correlaten van emotionele gevoeligheid, emotieregulatie en impulsiviteit openen nieuwe wegen voor toekomstig onderzoek en voorzien in waardevolle inzichten voor het verbeteren van behandelingen.

Om ons begrip van BPS te verbeteren hebben we in de volgende hoofdstukken 3, 4 en 5 een aantal suggesties uit het kritische review van hoofdstuk 2 toegepast. Ten eerste, studies 
die gezichtsherkenning onderzoeken rapporteren voor BPS patiënten een verhoogde gevoeligheid voor en moeite met het herkennen van negatieve gezichtsuitdrukkingen (sociale signalen voor dreiging en afwijzing), en een neiging om ambigue en neutrale gezichten negatief te interpreteren. Op basis van deze resultaten is het logisch dat de meeste onderzoeken zich richten op reacties tijdens uitsluitend negatieve stimuli, maar het blijft onduidelijk of de verhoogde reactiviteit zich beperkt tot negatieve emoties of dat het ook geldt voor andere valenties zoals positieve emoties, wat zou wijzen op een algemene verhoogde emotionele reactiviteit. Bovendien, gezien het feit dat seksueel trauma en relationele problemen vaak worden gemeld door BPS patiënten is het interessant om te onderzoeken of stimuli met erotische inhoud leidt tot intense emotionele reacties. Ten tweede, BPS heeft een hoge comorbiditeit met andere psychiatrische As I en As II stoornissen, voornamelijk stemming-, angst- (bijv. posttraumatische stress-stoornis), eetstoornissen en middelengebruik, en Cluster-C persoonlijkheidsstoornissen (d.w.z. ontwijkende, afhankelijke en obsessief-compulsieve persoonlijkheidsstoornis). Echter omdat een klinische controlegroep vaak ontbreekt in eerdere studies blijft het onduidelijk of bevindingen specifiek zijn voor BPS of algemene kenmerken van (persoonlijkheids) psychopathologie. Ten derde, de meeste voorgaande studies hebben een lage statistische power, mogelijk een van de belangrijkste oorzaken van de inconsistentie in de gerapporteerde bevindingen. Deze studies konden alleen zeer groot effect sizes detecteren en beperken daarmee de kans op het vinden van minder sterke effecten, ook al zijn die er wel degelijk. Om de statistische power te vergroten en kleinere effecten op te sporen moet men de steekproefomvang vergroten.

Het doel van dit proefschrift is het onderzoeken van de stimuluscategorie-specificiteit en diagnose-specificiteit van de neurale correlaten van emotionele gevoeligheid, emotieregulatie (Hoofdstuk 3 en 5) en impulsiviteit (Hoofdstuk 4) in BPS. Meer specifiek: 1) positieve en erotische stimuli zijn toegevoegd om de stimuluscategorie-specificiteit over stimuli met een verschillende emotionele inhoud te onderzoeken, 2) een Cluster-C persoonlijkheidsstoornis patiëntengroep is toegevoegd om de diagnose-specificiteit in vergelijking met andere persoonlijkheidsstoornissen te onderzoeken, en 3) de statistische power is vergroot in vergelijking met eerdere studies door de steekproefomvang te vergroten door middel van het werven van deelnemers uit verschillende (internationale) centra. Gebaseerd op voorgaand onderzoek was de hypothese dat BPS patiënten een hogere activiteit in de emotiegeneratie hersengebieden (amygdala en insula) laten zien wanneer ze passief kijken naar of reacties inhiberen van emotionele stimuli, in vergelijking met niet-patiënten en Cluster-C persoonlijkheidsstoornis patiënten. Bovendien verwachtten we dat BPS patiënten een verstoorde of in ieder geval andere activiteit vertonen in de regulerende of inhiberende hersengebieden (bijv. dorsolaterale prefrontale cortex, ventrolaterale prefrontale cortex en dorsale anterior cingulate cortex), tijdens 
het reguleren en inhiberen van emotionele stimuli, in vergelijking met niet-patiënten en Cluster-C persoonlijkheidsstoornis patiënten. Als de reacties in de hersenen van BPS patiënten specifiek zijn voor negatieve stimuli en bovendien diagnose-specifiek zijn voor BPS, dan zou er een verschil moeten zijn in de geactiveerde hersengebieden in vergelijking met positieve stimuli, en dit zou kenmerkend voor BPS zijn, wanneer deze groep niet alleen verschilt van niet-patiënten, maar ook van de Cluster-C persoonlijkheidsstoornis groep. Betreft de erotische stimuli verwachtten we dat deze vergelijkbare emotionele reacties zouden oproepen dan de negatieve stimuli bij de BPS patiënten.

Hoofdstuk 3 richtte zich op stimuluscategorie-specificiteit en diagnose-specificiteit van emotionele sensitiviteit en emotieregulatie in BPS. Tijdens het scannen werd een aangepaste versie van een klassiek emotieregulatie paradigma gepresenteerd, waarin de deelnemers afwisselden tussen het passief kijken naar sociale emotionele scenes en het reguleren van de emoties opgewekt door deze emotionele stimuli. Als regulatiestrategie werden de deelnemers geïnstrueerd om zich te realiseren dat ze veilig waren, geïnspireerd op schema therapie theorie. Conform onze hypothese van een hogere emotionele gevoeligheid in BPS, toonden de resultaten van passief kijken naar negatieve stimuli een verhoogde respons in de anterior insula (belangrijk voor emotiegeneratie) in BPS patiënten in vergelijking met niet-patiënten. Passief kijken naar negatieve stimuli leidde ook tot hogere activiteit in de temporoparietal junction en dorsolaterale prefrontale cortex in BPS. We konden echter niet de verhoogde amygdala respons op negatieve emotionele stimuli in BPS uit eerdere studies repliceren. Rekening houdend met de locatie van de amygdala, naast met lucht gevulde ruimtes waardoor het moeilijk kan zijn om amygdala responsen te detecteren (gevoelig voor artefacten en signaal uitval), werden extra tests uitgevoerd. Echter, de statistisch minder strenge amygdala regio-van-interesse analyse kon ook geen significante interactie-effecten detecteren, zelfs niet bij ongecorrigeerde $p$-waarde van .05. Andere mogelijk verstorende factoren zoals (vertraagde) gewenning van de amygdala, dissociatie die mogelijk de emotionele reactiviteit dempt, trauma, posttraumatische stress-stoornis, depressie, medicatie of lateralisatie konden deze nul-bevindingen ook niet verklaren. In overeenstemming met onze hypothese van verminderde emotieregulatie capaciteiten in BPS, vertoonden niet-patiënten tijdens het reguleren van de negatieve stimuli een hogere activiteit in de dorsale anterior cingulate cortex, dorsolaterale prefrontale cortex (belangrijk voor emotieregulatie), middelste temporale gyrus en bilaterale inferieur pariëtale lobule in vergelijking met passief kijken. BPS patiënten lieten deze toename van de activiteit niet zien wanneer ze geïnstrueerd werden om zich te realiseren veilig te zijn in vergelijking met de passief kijken conditie. Samengevat, tijdens passief kijken naar emotionele sociale stimuli vertoonden BPS patiënten een verhoogde respons in hersengebieden die belangrijk zijn voor generatie evenals regulatie van emoties, dat wijst op een uniek patroon van een toename van hersenactiviteit die betrokken is bij mentalisatie-processen (resoneren met 
mentale toestanden van anderen, temporoparietal junction) en emotionele bewustwording van lichamelijke responsen (anterior insula). Bij negatieve stimuli gaat dit gepaard met een verhoogde activiteit in regulatiegebieden (dorsolaterale prefrontale cortex). Echter toename van regulatiegebieden lijkt afwezig bij BPS patiënten wanneer ze expliciet geïnstrueerd worden te reguleren. Daarnaast vertoonden BPS patiënten een onvermogen om aandachtsgebieden (inferieure pariëtale lobule) te activeren tijdens emotieregulatie, wat kan interfereren met de cognitieve controle.

In Hoofdstuk 4 onderzochten we stimuluscategorie-specificiteit en diagnosespecificiteit van impulsiviteit in BPS. Tijdens het meten van hersenactiviteit voerden deelnemers een emotionele go/no-go taak uit met verschillende categorieën van emotionele sociale stimuli. Gedragsdata lieten zien dat BPS patiënten meer omissiefouten (nogo-reactie in go-trial) maakten dan niet-patiënten, dit neigt naar een aandachtsbeperking of een algemene inhibitie om het maken van commissiefouten (go-reactie in nogotrial) te voorkomen, terwijl de motor-inhibitie zelf werd gespaard, zoals weergegeven door een vergelijkbaar aantal commissiefouten tussen de groepen. Stimuluscategorie had geen effect op het aantal omissiefouten. In tegenstelling tot onze verwachtingen hebben we geen steun gevonden voor de hypothese van verminderde responsinhibitie in BPS. We konden de veranderende prefrontale activiteit uit eerdere studies betreffende impulsiviteit in BPS niet repliceren, in plaats daarvan toonden de neuroimaging resultaten een hogere activiteit in de frontale eye fields bij het inhiberen van negatieve stimuli in BPS patienten, terwijl niet-patiënten een lagere activiteit in de inferieure pariëtale lobule bij het inhiberen van negatieve stimuli lieten zien. Aangezien beide hersengebieden worden toegewezen aan het linker dorsale aandachtsnetwerk, die betrokken is bij topdown controle van aandacht over de hersengebieden van de sensorische cortex en het ventrale aandachtsnetwerk, speculeren we dat deze bevindingen wijzen in de richting van een verminderde top-down aandachtsbias en disfunctionele top-down controle over het ventrale aandachtsnetwerk in BPS. Ondersteund door de gedragsdata vertoonden BPS patiënten geen tekortkomingen in responsinhibitie tijdens de emotieverwerking per se, maar eerder vroege aandachtsproblemen voor emotionele informatie.

De resultaten van Hoofdstuk $\mathbf{3}$ met betrekking tot de stimuluscategorie-specificiteit duiden op een algemene emotionele gevoeligheid tijdens de bottom-up processen, terwijl de top-down emotieregulatie processen uniek waren voor negatieve stimuli. De resultaten met betrekking tot emotionele gevoeligheid waren niet specifiek voor negatieve stimuli, omdat de verhoogde respons in de emotiegeneratie hersengebieden ook aanwezig was tijdens passief kijken naar positieve stimuli, terwijl de resultaten met betrekking tot de emotieregulatie specifiek waren voor negatieve stimuli. In Hoofdstuk 4 toonden BPS patiënten een algemene responsiviteit over stimuluscategorieën in de inferieure pariëtale lobule, terwijl effecten in de frontale eye fields specifiek waren voor negatieve stimuli. Een 
significante interactie met positieve en erotische stimuli in de inferieure pariëtale lobule werd veroorzaakt door een modulatie van activiteit over stimuluscategorieën in niet-patiënten. Deze modulatie was afwezig in BPS patiënten, wat erop wijst dat ze niet werden beïnvloed door de stimuluscategorie. De frontale eye fields toonde hogere activiteit tijdens de presentatie van de negatieve stimuli in BPS vergeleken met de andere stimuluscategorieën, dit effect was niet aanwezig bij de niet-patiënten.

In Hoofdstuk $\mathbf{3}$ en $\mathbf{4}$ werd geen significant verschil gevonden met betrekking tot de emotiegeneratie en emotieregulatie hersengebieden en impulscontrole van de sociale emotionele stimuli voor de vergelijking van BPS patiënten versus Cluster-C persoonlijkheidsstoornissen patiënten, dat aangeeft dat deze resultaten niet diagnosespecifiek waren voor BPS. Lineairiteitsanalyses vonden steun voor een dimensionale benadering in plaats van een categoriale benadering met reacties van de Cluster-C persoonlijkheidsstoornis patiënten tussen de niet-patiënten en BPS patiënten in. Dit ondersteunt het idee dat Cluster-C persoonlijkheidsstoornis patiënten een aantal karakteristieken, maar in zwakkere mate, gemeen hebben met BPS.

FMRI is niet alleen geschikt voor het lokaliseren van structuren die betrokken zijn bij mentale processen, maar ook voor het bestuderen van verbindingen binnen en tussen hersennetwerken. Via functionele connectiviteit kan men de interactie tussen hersengebieden onderzoeken in plaats van hersengebieden in isolatie. Grote netwerken van hersenstructuren voeren samen een taak uit; één hersengebied ontvangt input vanuit andere hersengebieden en stuurt output naar nog andere hersengebieden. Omdat emotie-disregulatie in BPS in verband wordt gebracht met een verminderde amygdala-prefrontale inhiberende netwerk hebben we in Hoofdstuk 5 fMRI tijdens rust gebruikt om aanhoudende effecten van emotieregulatie op de amygdala intrinsieke functionele connectiviteit te onderzoeken. FMRI tijdens rust werd gemeten voor en na de emotieregulatie taak uit Hoofdstuk 3. In vergelijking met niet-patienten werd verwacht dat BPS patiënten een zwakkere toename van functionele connectiviteit tijdens rust tussen de amygdala en de prefrontale gebieden zouden vertonen na de emotieregulatie, dat wijst op problemen met emotieregulatie. Hoewel gedragsresultaten van emotionele ervaring onmiddellijk na de passief kijken en veilig conditie een soortgelijke verandering van emotionele toestand in BPS patiënten en niet-patiënten toonden als gevolg van cognitieve regulatie, vertoonden BPS patiënten een andere functionele connectiviteit tijdens rust na de emotieregulatie taak. In overeenstemming met onze hypothese lieten BPS patiënten, in vergelijking met niet-patiënten, geen toename zien in post-taak amygdala intrinsieke functionele connectiviteit met hersengebieden essentieel voor emotieregulatie waaronder de mediale, dorsolaterale en ventrolaterale prefrontale cortex en superieure temporale gyrus. BPS patiënten vertoonden een post-taak verminderde amygdala functionele connectiviteit tijdens rust met de posterior cingulate cortex betrokken bij zelf-referentie 
en autobiografisch geheugen, en verhoogde amygdala functionele connectiviteit tijdens rust met de superieure pariëtale lobule betrokken bij aandacht en episodisch geheugen. Een vergelijking met de Cluster-C persoonlijkheidsstoornis patiënten suggereerde dat de directe regulering van de amygdala via de ventromediale prefrontale cortex niet specifiek is voor BPS, maar lijkt gemeenschappelijk te zijn voor persoonlijkheidsstoornissen met overlappende emotionele en interpersoonlijke problemen.

Naast de sterke punten van dit proefschrift moeten er ook een aantal beperkingen worden erkend. Ten eerste, hebben we enkel vrouwen getest wat de generaliseerbaarheid van onze resultaten naar mannen beperkt. In de geestelijke gezondheidszorg instellingen wordt BPS drie keer vaker gediagnosticeerd bij vrouwen dan bij mannen. Daarnaast laat het klinische beeld van BPS sekseverschillen zien, waarbij BPS vrouwen meer internaliserend emotioneel gedrag tonen en BPS mannen externaliserend, ongeremd en reactief agressief gedrag. Toekomstig onderzoek moet de mogelijke rol van geslacht in BPS verduidelijken. Ten tweede, veel BPS patiënten gebruiken medicatie, het gebruik van medicatie kan echter een belangrijke verstorende factor zijn bij het bestuderen van hersenactiviteit. Wij hebben patiënten met medicatie niet uitgesloten om een representatieve en ernstige klinische steekproef te werven. Om te controleren op mogelijke effecten van medicatie, en omdat het toevoegen van medicatie als covariaat de variantie geassocieerd met de groepsverschillen verwijdert, hebben wij aanvullende analyses gedaan binnen de BPS groep (met medicatie versus zonder medicatie). Voor de gerapporteerde clusters werden geen significante effecten van medicatie binnen de BPS groep gevonden, wat aangeeft dat de resultaten robuust waren voor medicatie-effecten. Het moet worden erkend dat het niet duidelijk is of de veronderstelling om patiënten enkele weken voor het scannen te verzoeken geen medicatie meer te gebruiken resulteert in 'normale' hersenactiviteit op het moment van scannen. Voor sommige medicijnen kan het enkele maanden duren voordat ze zijn uitgewerkt en zelfs uitgewerkte medicatie kan nog steeds blijvende effecten in de hersenen hebben veroorzaakt. Bovendien, kan het eisen van patiënten om te stoppen met hun medicatie ethisch problematisch zijn en draagt het naar alle waarschijnlijkheid bij aan een vorm van steekproefvertekening. Een andere complicatie is dat vaak verschillende combinaties van soorten medicatie worden gebruikt door patiënten, dit maakt het vrijwel onmogelijk om het effect van een enkel type medicatie te isoleren. Om meer inzicht te krijgen in de mogelijke werking van medicatie, moet medicatie adequater worden gedefinieerd (type, aantal en dosis).

Emotionele gevoeligheid, emotieregulatie en impulsiviteit bij BPS patiënten zijn belangrijke onderwerpen in neuroimaging onderzoek. In dit proefschrift toonden we bewijs voor algemene emotionele gevoeligheid en verminderde emotieregulatie voor negatieve stimuli in BPS. Daarnaast lieten BPS patiënten geen aanhoudende effecten zien na de emotieregulatie taak in hersengebieden betrokken bij emotieregulatie. Bovendien werd er 
geen steun gevonden voor tekortkomingen van responsinhibitie tijdens emotieverwerking. Interessant is dat huidige studies wijzen op mogelijke aandachtsproblemen in BPS tijdens emotieregulatie en impulscontrole. In BPD patiënten was hersenactiviteit van gebieden betrokken bij emotionele gevoeligheid en in de inferieure pariëtale lobule tijdens impulscontrole niet stimulus specifiek, terwijl hersenactiviteit in gebieden betrokken bij emotieregulatie en de frontale eye fields tijdens impulscontrole specifiek waren voor negatieve stimuli. Over het algemeen toonden de Cluster-C persoonlijkheidsstoornispatiënten een mate van hersenactiviteit tussen BPS patiënten en niet-patiënten in, wat de dimensionale benadering ondersteunt. Begrijpen van onderliggende hersenmechanismen kan belangrijke inzichten in BPS geven en kan waardevol zijn om therapieën en uiteindelijk persoonlijke condities van BPS patiënten en maatschappelijke lasten te verbeteren. Gebaseerd op verhoogde activiteit in hersengebieden die belangrijk zijn voor de generatie (insula) en regulatie (dorsolaterale perfrontale cortex) tijdens het passief kijken naar emotionele stimuli (Hoofdstuk 3), en een andere hersenactiviteit in aandachtsgerelateerde hersengebieden (inferieure pariëtale lobule en frontale eye fields) tijdens impulscontrole (Hoofdstuk 4) in BPS patiënten in vergelijking met niet-patienten, kunnen dit regiosvan-interesse zijn wanneer gekeken naar veranderingen in hersenactiviteit tijdens en na psychotherapie, real-time fMRI neurofeedback en transcraniële magnetische stimulatie om hersenactiviteit te beheersen en effectieve regulatie en inhibitie strategieën te leren. 



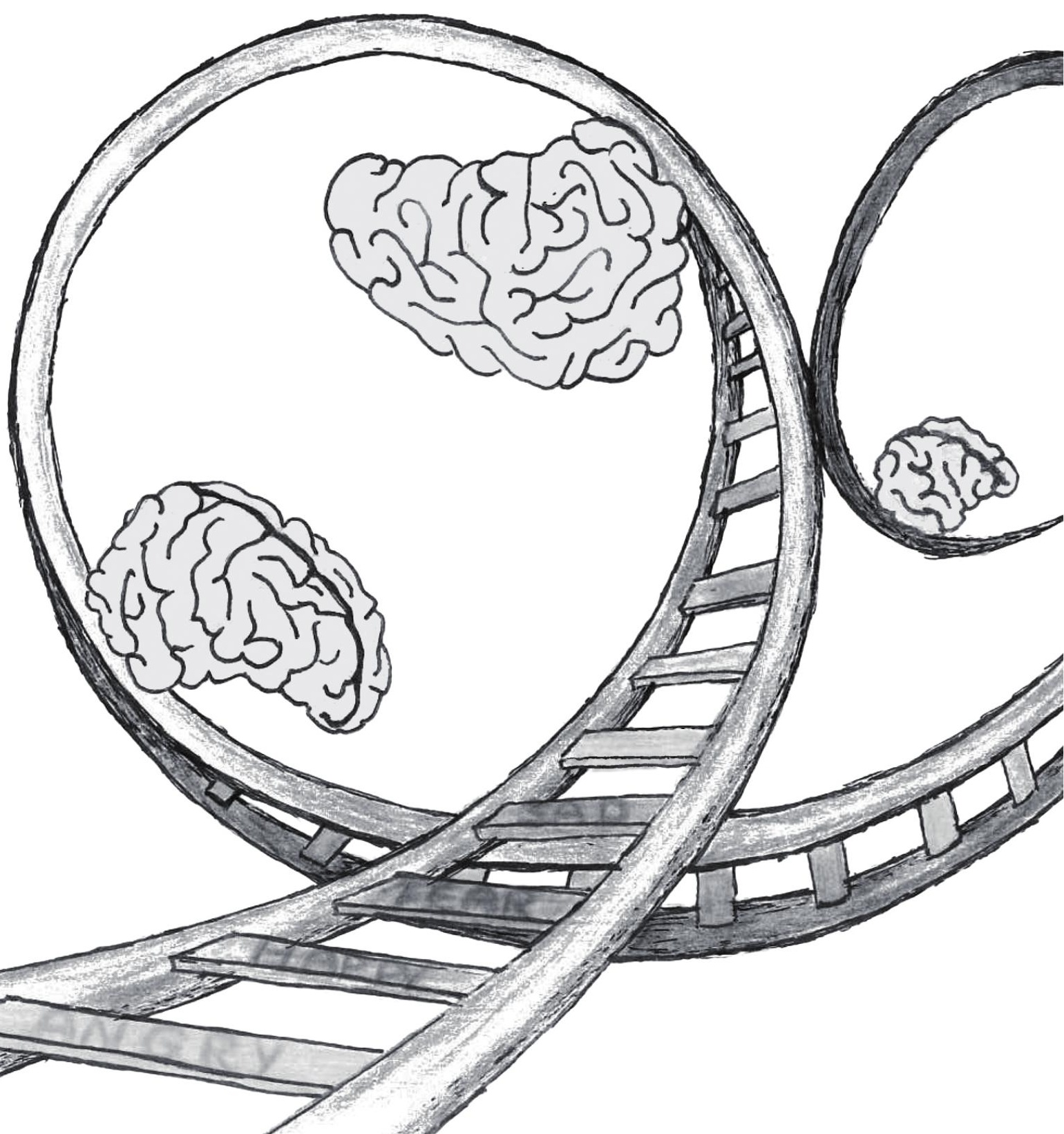


Dankwoord

Acknowledgements 

Na 5 jaar is het proefschrift dan eindelijk af. Jaren waarin ik veel leerzame en leuke ervaringen heb meegemaakt, met de nodige ups en downs zoals dat hoort bij onderzoek. De onderzoeken beschreven in dit proefschrift heb ik niet alleen gedaan. Daarom op deze plek een woord van dank aan iedereen die me heeft gesteund en heeft bijgedragen aan dit proefschrift.

Allereerst gaat mijn dank uit naar mijn promotoren en copromotor, Arnoud, Rainer en Nicolette. Bedankt dat jullie mij de mogelijkheid en het vertrouwen gaven tot het doen van dit promotieonderzoek.

Beste Arnoud, ik heb veel van je geleerd de afgelopen jaren. Ik heb bewondering voor je enorme kennis, gedrevenheid, enthousiasme en kritische blik. Bedankt voor je altijd snelle reacties en je hulp bij de (door mij gevreesde) lineair mixed model analyses. Ondanks dat je in Amsterdam zit was je nog altijd even toegankelijk en betrokken. Ik vind het fijn dat we onze samenwerking kunnen voortzetten en hoop nog meer van je te leren. Misschien kan ik jou dan af en toe nog een nieuwe hersengebieden leren, één per jaar was toch het doel.

Dear Rainer, thank you for your valuable help concerning the choices of the most appropriate analysis of the data, programming the possibility of a 2-between factor model, but mostly for the motivational meetings. I admire your skill to explain something difficult in a very understandable and easy manner.

Beste Nicolette, ik bewonder je enorme parate kennis (inclusief bijbehorende studies, journal en jaartal) en hoe je een boodschap in een goed verhaal kan overbrengen. Bedankt voor je onmisbare inzichten tijdens de analyses en je creativiteit bij het schrijven van de manuscripten. We zijn een goed team, met mijn planningsvaardigheden en nauwkeurige uitvoer en de door jou snelle koppeling aan theorieën en samen bedachte hypothesen vullen we elkaar goed aan. Samen met Arnoud heb je me kritisch laten nadenken over de relevantie en implicaties van het neuroimaging onderzoek.

I also would like to thank the members of the ORA research group. Thank you Gitta, Gregor, Debbie, Simon, Andreas, Alena, Oliver and Bastian for your support, help with participant recruitment and measurements, critical suggestions and knowledge. It was inspiring to collaborate with you all to succeed this multicenter study.

Members of the evaluation committee, thank you very much for taking the time and effort to read and evaluate this dissertation and your willingness to participate in the defense.

Bij het uitvoeren van een promotie-onderzoek komt veel kijken, en gelukkig kreeg ik van veel kanten ondersteuning. Vincent bedankt voor je hulp bij de connectiviteitsanalyses. Ik hoop dat ik ook in de toekomst nog af en toe gebruik mag maken van jouw expertise. 
Armin and Brainvoyager support, thank you very much for the times that you helped me with Brainvoyager.

Mannen van de instrumentatie, met name Erik, Jacco, Ron en Richard, bedankt voor de persoonlijke begroetingen in de gangen en gesprekken in de koffiehoek, maar ook voor jullie ondersteuning bij de eyetracker. Michiel bedankt voor je hulp bij het programmeren in matlab, wanneer ik weer eens overhoop lag met het scripten.

Zonder hulp van onderzoeksassistenten was dit project niet mogelijk geweest, jullie ook hartelijk dank. Michelle en Rosanne bedankt voor jullie logistieke hulp in het begin van dit project, Lisette en Nina voor het uitvoeren van de metingen bij de deelnemers van het GroupST project, Brigit voor je inzet bij het werven van de controle patiënten en Annie voor het afnemen van de soms heftige trauma interviews.

Lindy, Jessie, Marionne en Carolien, bedankt voor alle ondersteuning met een glimlach. Het is me een genoegen jullie buurvrouw te zijn.

Omdat je een groot deel van je tijd op je werk doorbrengt zijn een goede sfeer en de benodigde gezelligheid essentieel.

Aan mijn vroegere en huidige kamergenootjes, jullie maken het een plezier om naar het werk te gaan. Rachelle, Ramon en Martijn bedankt dat jullie mij 'illegaal' tolereerde als $4^{\text {de }}$ persoon op de kamer. letsje langer dan de bedoeling was, en Martijn ook nu ben je (voorlopig) nog niet van me af $:$.). Bedankt voor de interessante gespreken en Rachelle voor het introduceren van Muffin O'Clock.

Beste collega's van CPS en de CP-ers in het bijzonder: bedankt voor jullie collegialiteit, inspiratie en wetenschappelijke inzichten, maar ook voor de lunchpauzes, borrels en uitjes; voor de juiste 'work and fun' balance binnen én buiten de werkuren. Bedankt dat jullie zo'n fijne collega's zijn, waarvan sommigen gaande de tijd vrienden geworden zijn. Marjolein, wij kunnen kletsen over van alles en nog wat, zeker onder het genot van een pizza. Ik ben blij dat je terug bent. Lorraine, Pim, Lotte en Andrea bedankt voor de leuke gesprekken en goede verhalen. Jullie zijn top! Melanie zonder jou zou het toch extra moeite kosten om na het werk nog de sportschool in te duiken. Ook dank voor de enkele ritjes naar de eerste hulp. Manuela ik ben blij dat wij nog steeds contact hebben en de sushi-dates houden we er in. Franca bedankt voor de gezellige wijnavondjes, het klinken op de successen en je energie.

Mijn twee paranimfen, Conny \& Suzanne. Ik ben blij dat jullie mij op deze bijzondere dag achter mij staan. Conny, mijn eerste kamergenootje, super bedankt voor het warme welkom op de UM. Je staat altijd voor samen iedereen klaar, al lachend horen we je door de gang rennen. Daarnaast partner in fMRI, we hebben wat uurtjes doorgebracht tijdens het scannen. Suzanne, je bent een sterke vrouw en ik zal nooit vergeten dat je vertelde dat je zwanger was terwijl je de week ervoor nog glashard zei dat het nog niet op de planning stond $(;)$. 
Er zijn een aantal vrienden die ik in het bijzonder wil bedanken voor hun steun.

Linda, wij zijn al heel wat jaren vriendinnetjes, ondanks de beperkte live contacten, bedankt voor het zijn van een lieve vriendin. Lisette in Amsterdam en Judith inmiddels uitgevlogen naar Duitsland, ik hoop dat onze vriendschap nog lang mag duren. Berna \& Michel het is dat Groningen niet om de hoek ligt, anders stond ik vaker op de stoep.

Ten slotte wil ik mijn familie bedanken. Pap, Mam, Fran \& Vin dankjewel voor alle steun en een luisterend oor wanneer nodig. Ook dank voor het ontwerp van de afbeelding op omslag. Ondanks dat jullie misschien niet altijd weten waar ik nu precies mee bezig ben, hebben jullie altijd achter me gestaan en me gestimuleerd, met de benodigde raad en daad. Het was heerlijk soms even de boel de boel te laten in Maastricht en te relaxen op de boerderij. 


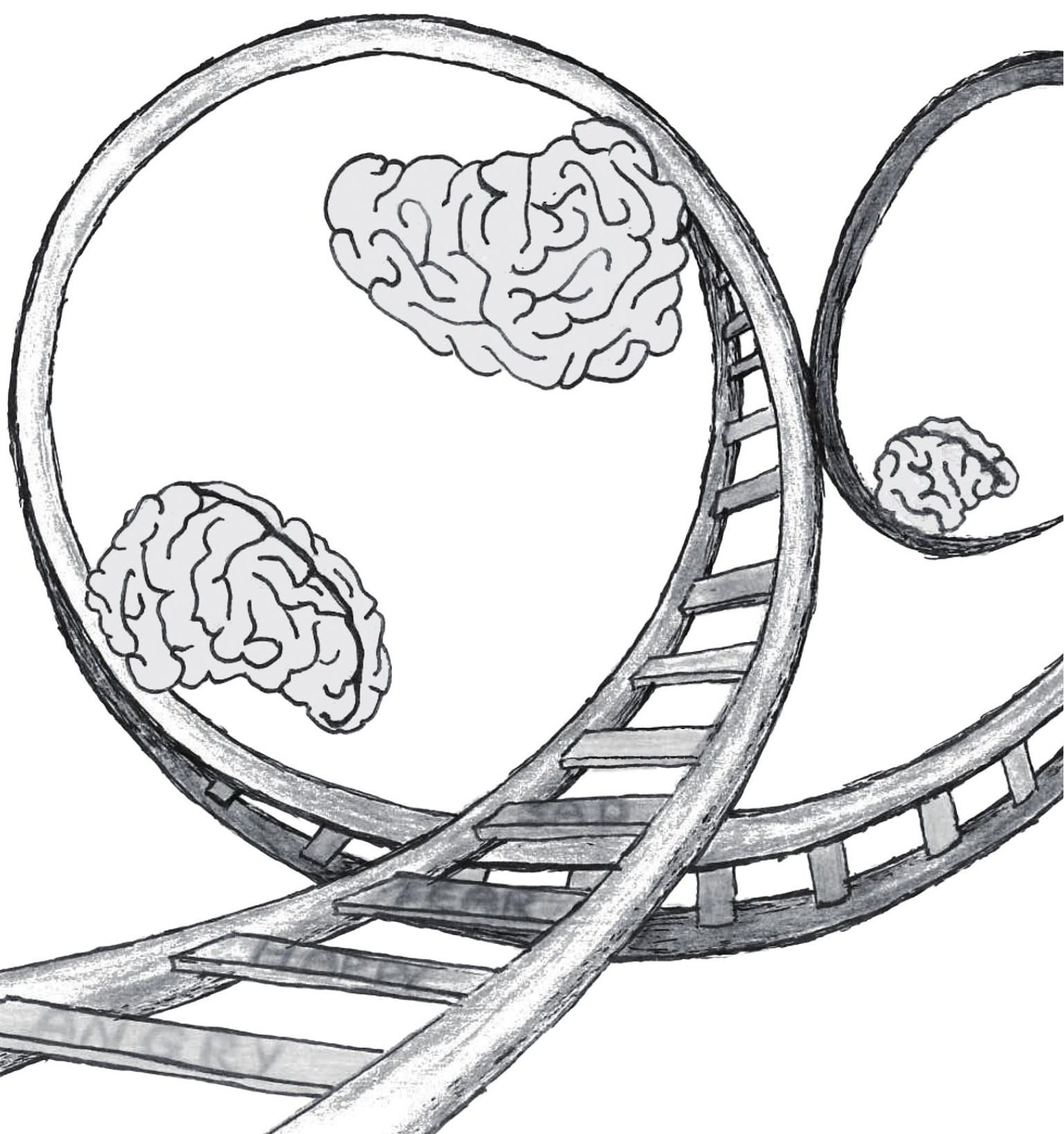


List of Publications 



\section{Journal articles}

Van Zutphen, L., Siep, N., Jacob, G.A., Goebel, R. \& Arntz, A. (2015). Emotional sensitivity, emotion regulation and impulsivity in borderline personality disorder: A critical review of fMRI studies. Neuroscience and Biobehavioral Reviews, 51, 64-76.

Baczkowski, B.M.*, van Zutphen, L.* , Siep, N., Jacob, G.A., Domes, G., Maier, S. Sprenger, A., Senft, A., Willenborg, B., Tüscher, O., Arntz, A. \& van de Ven, V. (2016). Deficient amygdalaprefrontal intrinsic connectivity after effortful emotion regulation in borderline personality disorder. European Archives of Psychiatry and Clinical Neuroscience. doi: 10.1007/s00406-016-0760-z

* joint first authorship

\section{Submitted Manuscripts}

Van Zutphen, L., Siep, N., Jacob, G.A., Domes, G., Sprenger, A., Willenborg, B., Goebel, R. \& Arntz, A. (n.d.) Always on guard: Emotion regulation in borderline personality disorder compared to non-patients and cluster-c personality disorder patients. Manuscript submitted for publication

Van Zutphen, L., Siep, N., Jacob, G.A., Domes, G., Sprenger, A., Willenborg, B., Goebel, R., Tüscher O. \& Arntz, A. (n.d.). Impulse control under emotion processing: an fMRI investigation of borderline personality disorder compared to non-patients and cluster-c personality disorder patients. Manuscript submitted for publication

Kaiser, D., Jacob, G.A., van Zutphen, L., Siep, N., Sprenger, A., Tuschen-Caffier, A., Senft, A., Arntz, A. \& Domes, G. (n.d.). Biased attention to facial expression of anger in borderline personality disorder: an eyetracking study. Manuscript submitted for publication

Tonnaer, F., Siep, N., van Zutphen, L., Arntz, A. \& Cima, M. (n.d.). Anger provocation in violent offenders leads to emotion dysregulation. Manuscript submitted for publication

Goodwin, G.M., Holmes, E.A., Andersson, E., Browning, M., Jones, A., Lass-Hennemann, J., Månsson, K.N.T., Moessnang, C, Sanchez, A., van Zutphen, L., \& Visser, R.M. (2017). Proceedings "From Neuroscience to Evidence Based Psychological Treatments The Promise and the Challenge", ECNP 20-21 March 2016, Nice, France. European Neuropsychopharmacology, Submitted

\section{Manuscripts in preparation}

Van Zutphen, L.* , Maier, S.* Siep, N., Jacob, G.A., Tüscher, O., Tebartz van Elst, A., Zeeck, E., Arntz, A., O'Connor, M-F., Stamm, H., Hudek, M. \& Joos, A. Intimate stimuli induce frontopariatal activation in anorexia nervosa. Manuscript in preparation

* joint first authorship 


\section{Conference Presentations}

Van Zutphen, L., Siep, N., Jacob, G.A., Domes, G., Maier, S., Sprenger, A., Willenborg, B., Tüscher, O., Goebel, R. \& Arntz, A. (March, 2016). Neural correlates of response inhibition in borderline personality disorder: stimulus specificity and diagnosis specificity. Poster presentation at ECNP Neuroscience and Psychotherapy Meeting 2016, Nice, France

Van Zutphen, L., Siep, N., Jacob, G.A., Domes, G., Maier, S., Sprenger, A., Willenborg, B., Tüscher, O., Goebel, R. \& Arntz, A. (April, 2015). Neural correlates of emotion dysregulation in borderline personality disorder: stimulus specificity and diagnosis specificity. Poster presentation at Social and Affective Neuroscience Annual Conference, Boston, Massachusetts, USA

Van Zutphen, L., Siep, N., Jacob, G.A., Domes, G., Maier, S., Sprenger, A., Willenborg, B., Tüscher, O., Goebel, R. \& Arntz, A. (September, 2013). Neural correlates of emotional impulsivity in borderline personality disorder. Oral presentation at the XIII International Congress on the Disorders of Personality. In symposium Impulsivity in borderline personality disorder, Siep, N. (chair) in Copenhagen, Denmark

Van Zutphen, L., Siep, N., Jacob, G.A., Domes, G., Maier, S., Sprenger, A., Willenborg, B., Tüscher, O., Goebel, R. \& Arntz, A. (September, 2012). Emotion dysregulation in Borderline Personality Disorder: an fMRI study. Oral presentation at the $2^{\text {nd }}$ International Congress on Borderline Personality Disorder and Allied Disorders. In symposium Recent advances in borderline personality disorder research, Herpertz, S.C. (chair) in Amsterdam, the Netherlands 



\section{About the Author}

Linda van Zutphen was born in Emmeloord (the Netherlands) on November $9^{\text {th }} 1984$. She graduated from secondary school at Zuyderzee College in Emmeloord in July 2003, after which she started her study Human Movement Science at the University of Groningen. She received her master's degree on August 31 2008. To study the relationship between brain and behavior, she subsequently started the research master Cognitive Neuropsychology at the Free University Amsterdam, where she obtained her second master's degree on August 31 2010. In August 2011, she began her PhD project at the Department of Clinical Psychological Science at Maastricht University. She is currently working as a post-doctoral researcher in the same department, where she is continuing her research on change in brain activation of emotion regulation and impulsivity during the course of psychotherapy in borderline personality disorder. 